\title{
Base Mediated Cyclopentannulation of Ynones with Amino Crotonates for Regio- \& Stereoselective Synthesis of Pentafulvenes and Cyclopenta[c]quinolines
}

Attunuri Nagireddy, ${ }^{\mathrm{a} \ddagger}$ Maneesh Kumar Reddy Singam, ${ }^{\mathrm{a} \ddagger}$ Jagadeesh Babu Nanubolu ${ }^{\mathrm{b}}$ and Maddi Sridhar Reddy ${ }^{a *}$

aDepartment of OSPC, CSIR-Indian Institute of Chemical Technology, Habsiguda, Hyderabad 500007, India. Academy of Scientific and Innovative Research, New Delhi 110001, India. bAnalytical Department, CSIR-IICT, Hyderabad-500007.

*E-mail: msreddy@iict.res.in, msreddy@,cdri.res.in

\section{Table of Contents}

I. Copies of ${ }^{1} \mathrm{H},{ }^{13} \mathrm{C},{ }^{19} \mathrm{~F}$ NMR and HRMS spectra S1-S88

II. X-ray crystallography data S89-S91 
Figure S1: ${ }^{1} \mathrm{H},{ }^{13} \mathrm{C},{ }^{19} \mathrm{~F}$ NMR and HRMS spectra's of $2 \mathrm{a}-2 \mathrm{j}$, 3aa-3wa, 3ab-3aj, 4a-4h and 5.

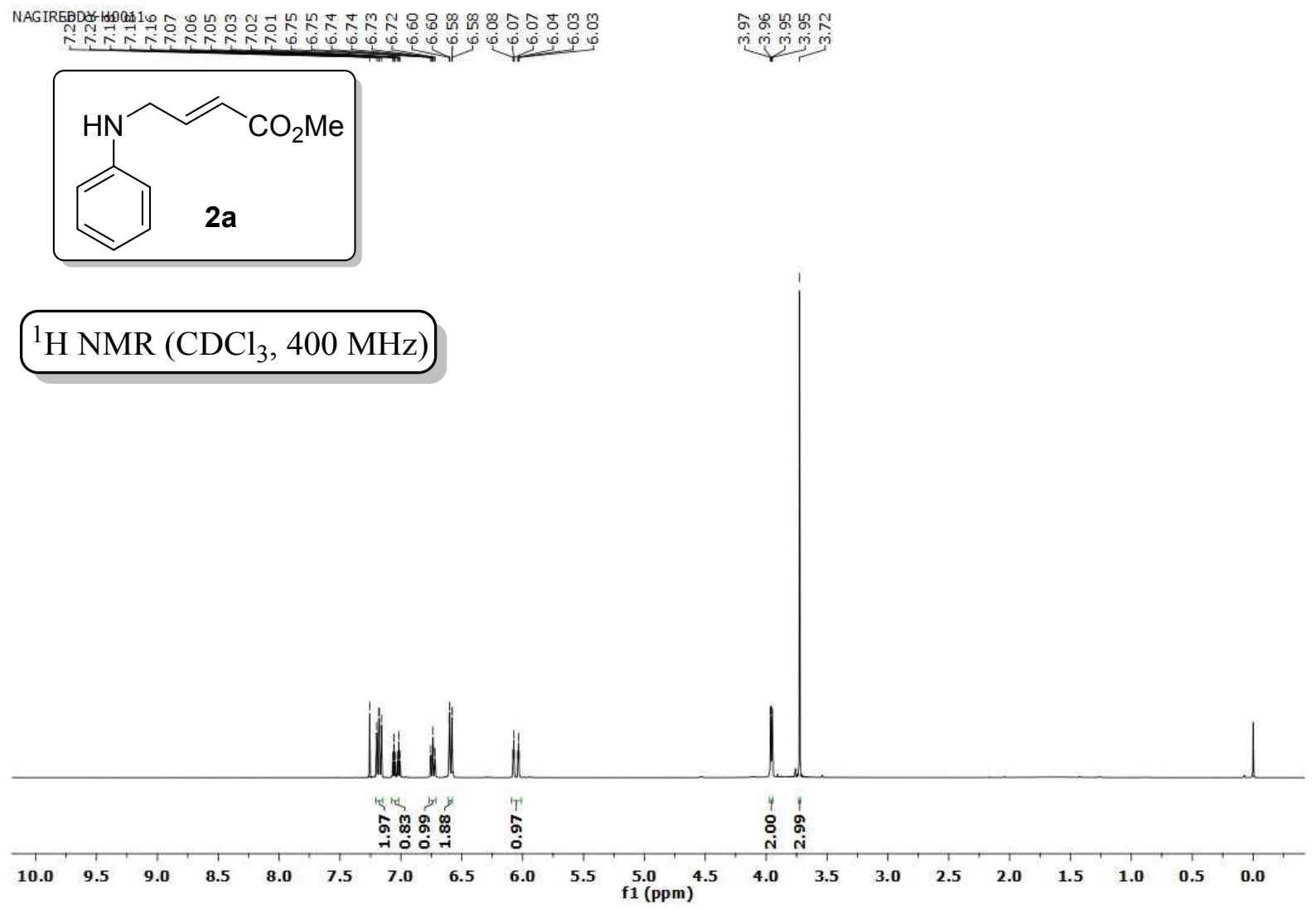


NAGIREDDY-H0011

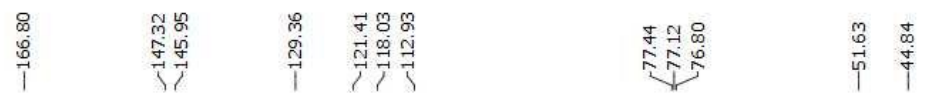

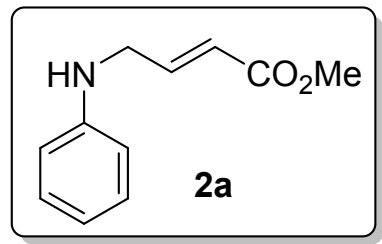

${ }^{13} \mathrm{C} \mathrm{NMR}\left(\mathrm{CDCl}_{3}, 100 \mathrm{MHz}\right)$
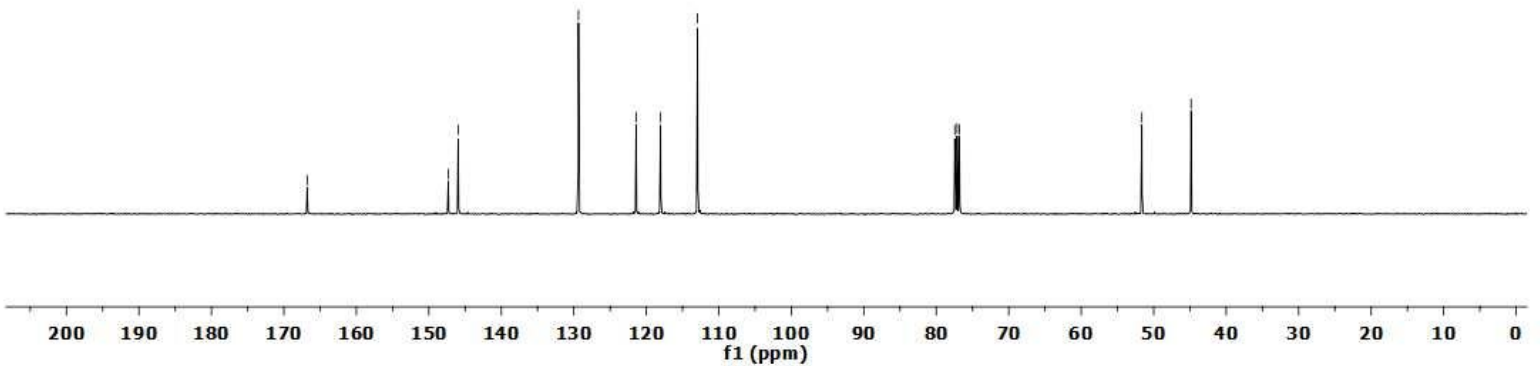

MSR_16_D_192

CSIR-Indian Inistiute of Chemical Technology

19DEC_2019_1 15 (0.293) AM2 (Ar,22000.0,0.00,0.00): ABS: Cm (11:37-11:10+33:44))

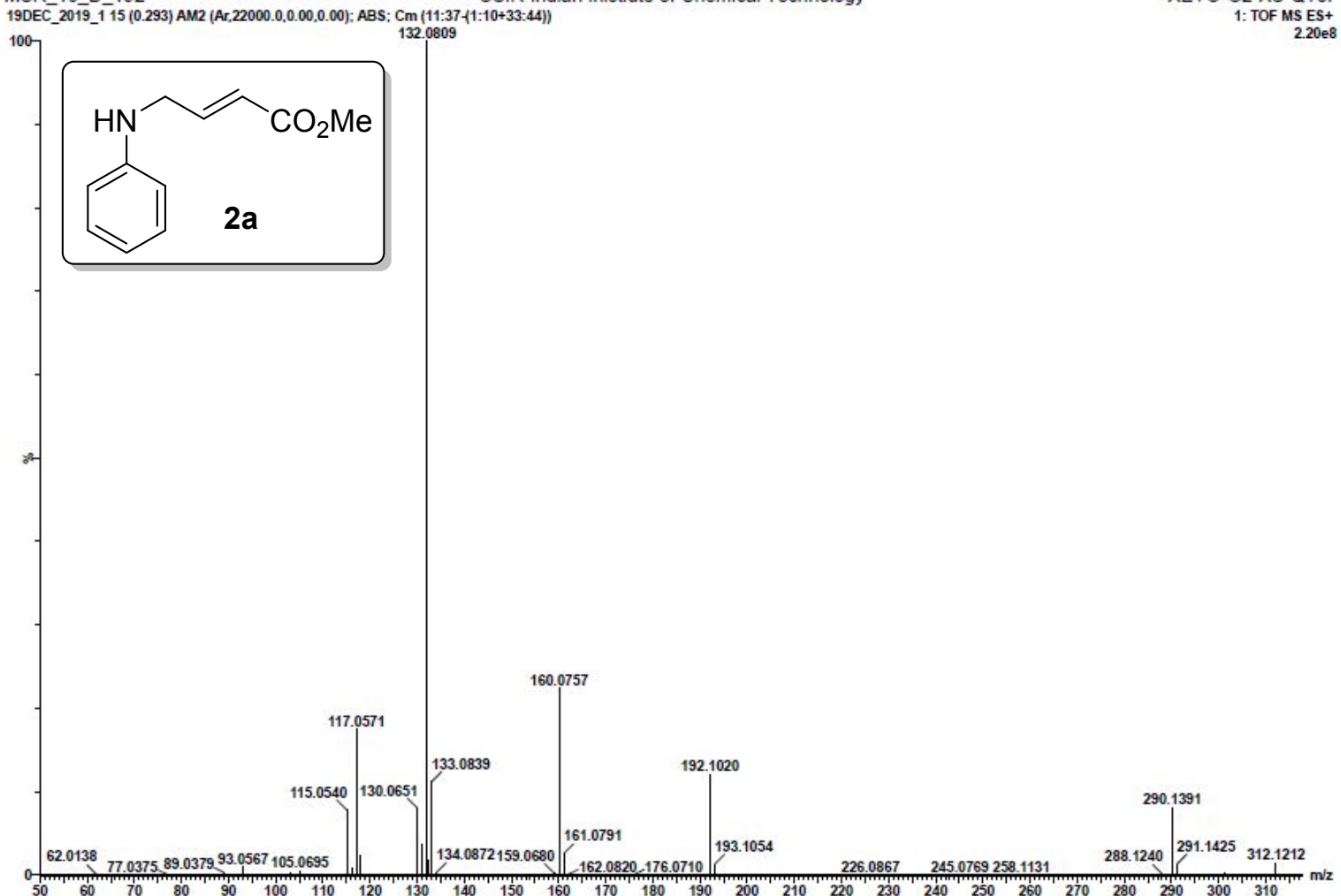




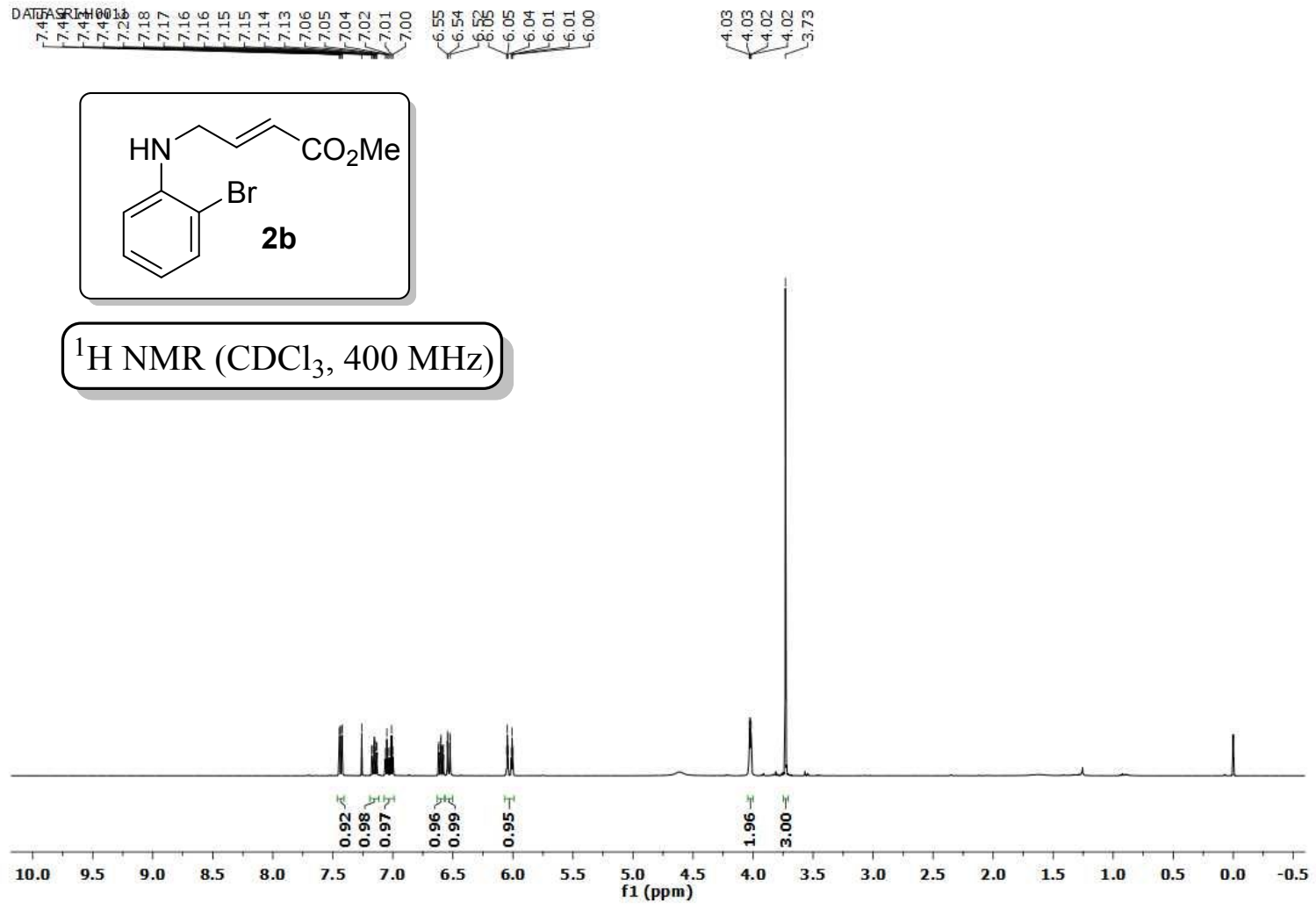

NAGIREDDY-H0011

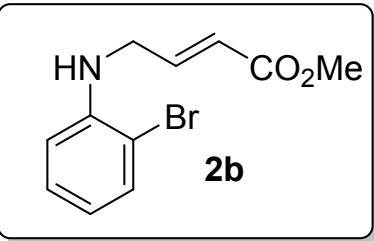

${ }^{13} \mathrm{C} \mathrm{NMR}\left(\mathrm{CDCl}_{3}, 100 \mathrm{MHz}\right)$

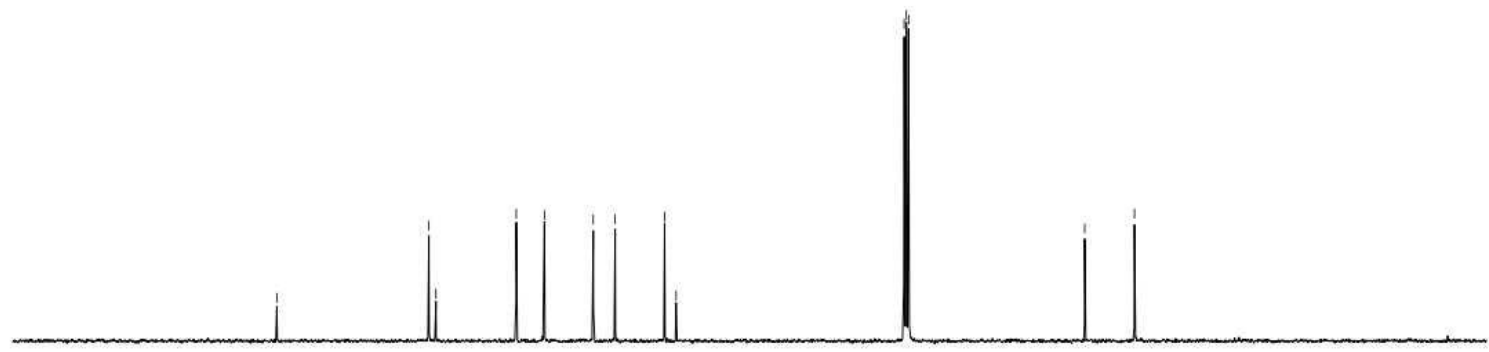

$\begin{array}{lllllllllllllllllllll}200 & 190 & 180 & 170 & 160 & 150 & 140 & 130 & 120 & 110 & \begin{array}{c}100 \\ \mathrm{f} 1(\mathrm{ppm})\end{array} & 90 & 80 & 70 & 60 & 50 & 40 & 30 & 20 & 10 & 0\end{array}$ 


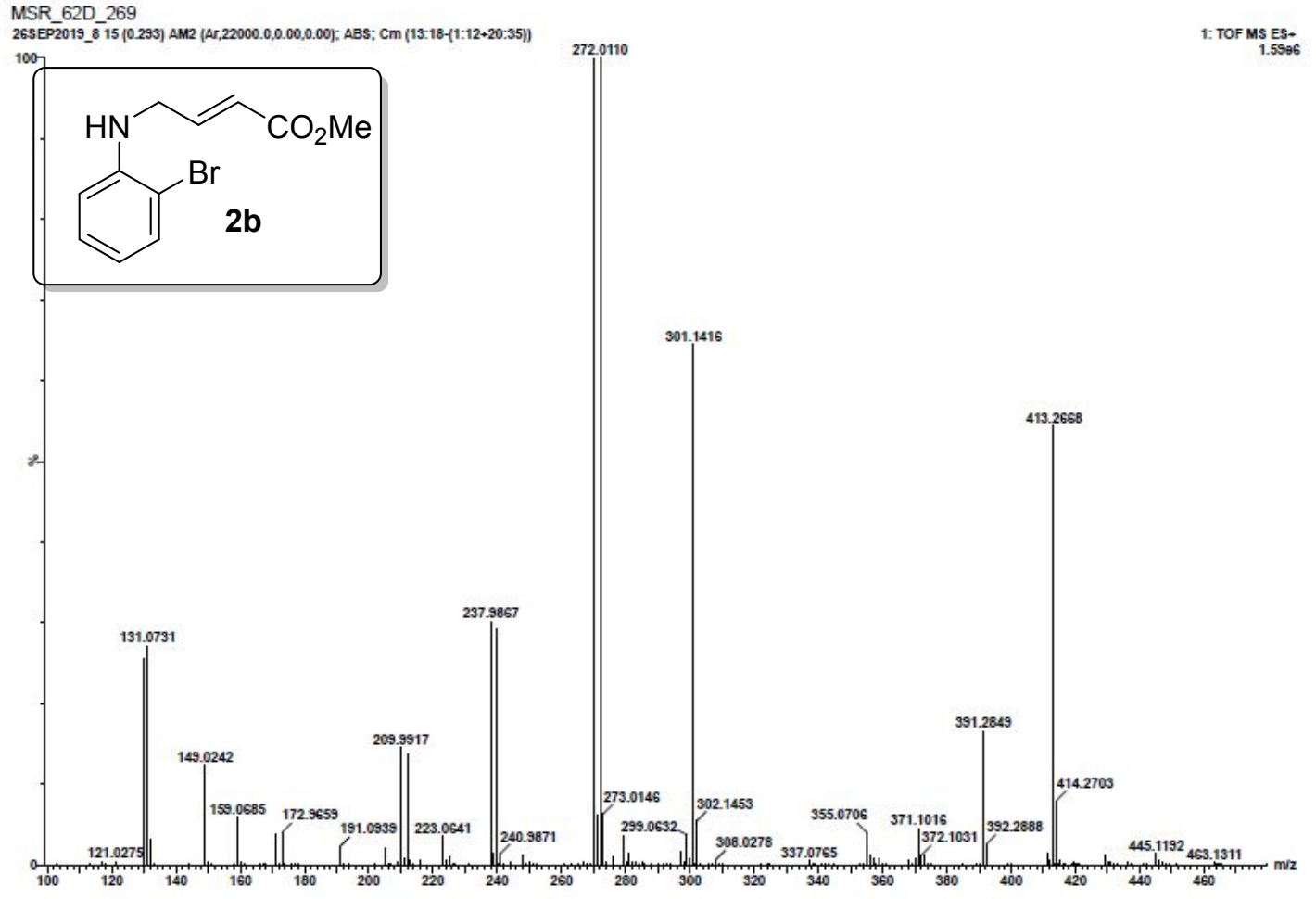


NAGIREDDY-M0034

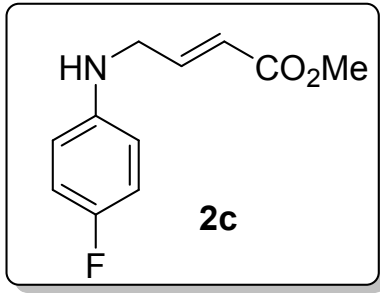

${ }^{1} \mathrm{H} \mathrm{NMR}\left(\mathrm{CDCl}_{3}, 400 \mathrm{MHz}\right)$

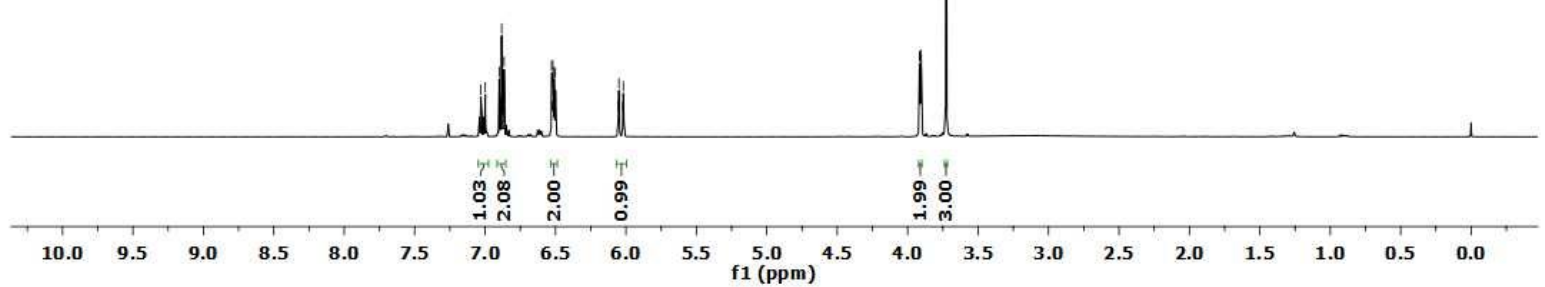

DATTHATRI-M0034

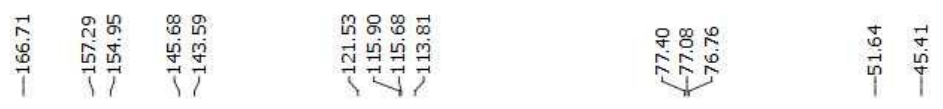

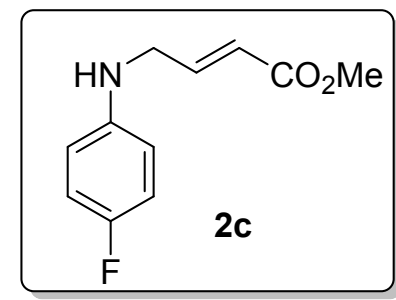

${ }^{13} \mathrm{C} \mathrm{NMR}\left(\mathrm{CDCl}_{3}, 100 \mathrm{MHz}\right)$

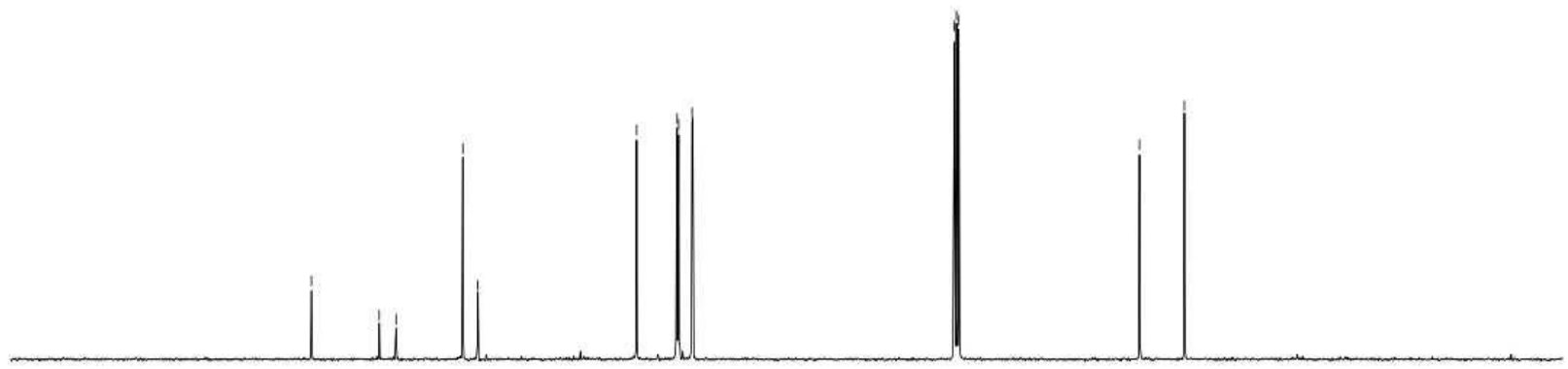

$\begin{array}{lllllllllllllllllllll}200 & 190 & 180 & 170 & 160 & 150 & 140 & 130 & 120 & 110 & 100 & 90 & 80 & 70 & 60 & 50 & 40 & 30 & 20 & 10 & 0\end{array}$ 


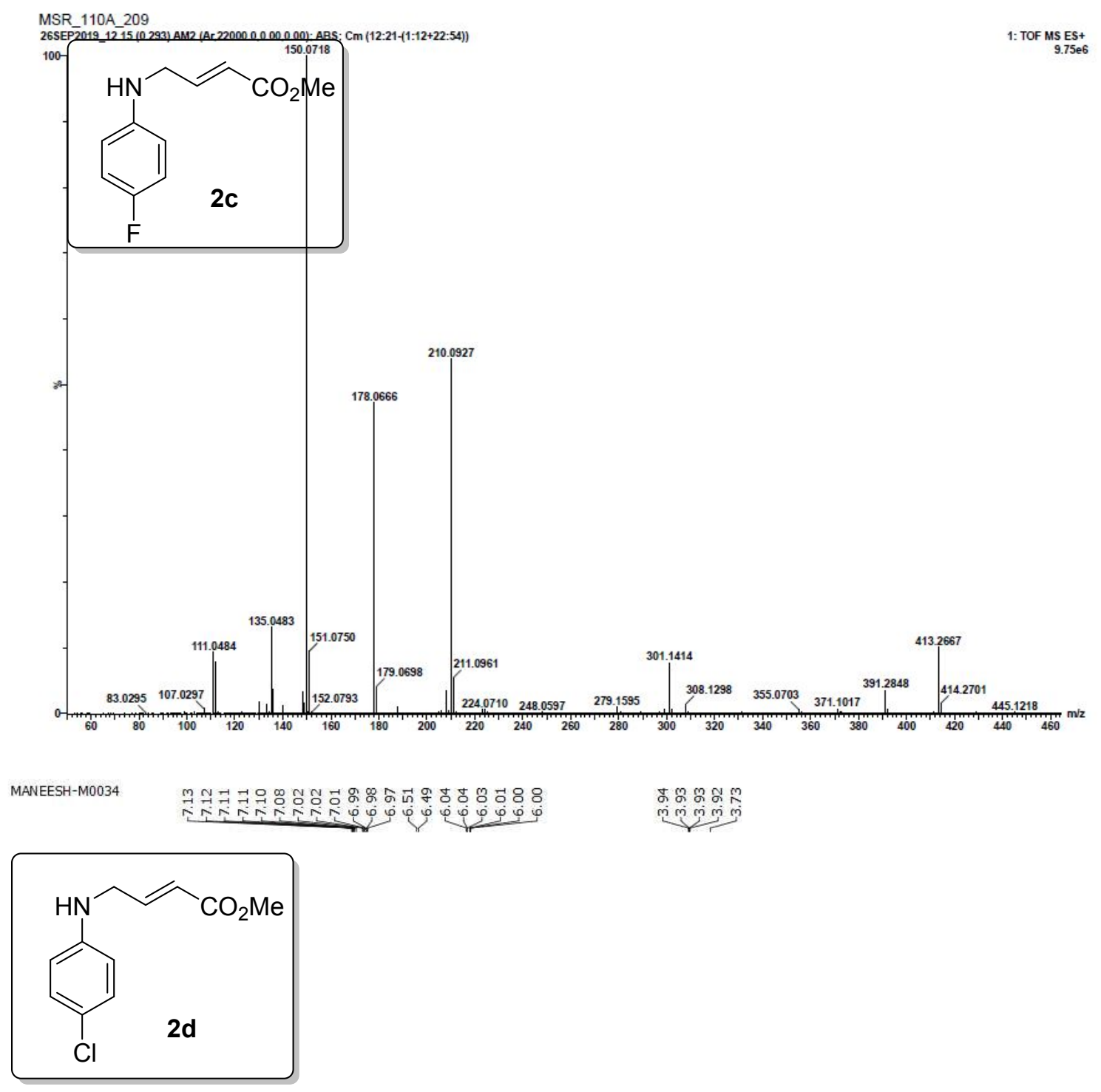

${ }^{1} \mathrm{H}$ NMR $\left(\mathrm{CDCl}_{3}, 400 \mathrm{MHz}\right)$

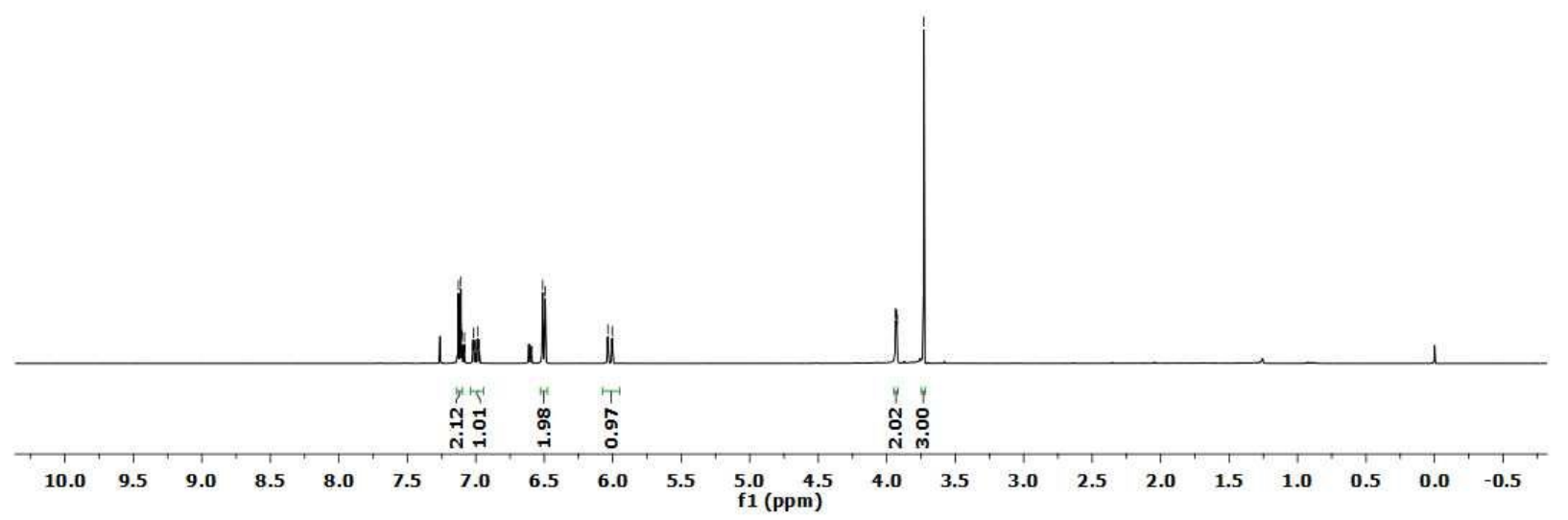


MANEESH-M0034
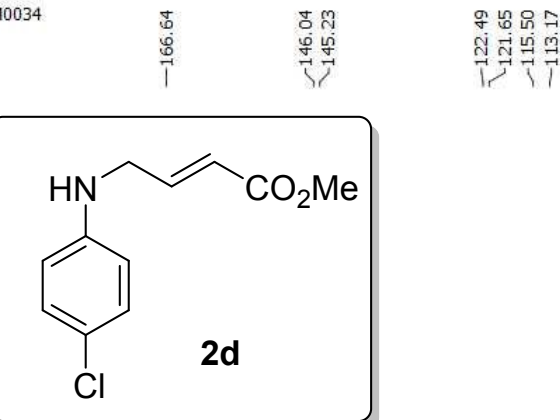

${ }^{13} \mathrm{C} \mathrm{NMR}\left(\mathrm{CDCl}_{3}, 100 \mathrm{MHz}\right)$
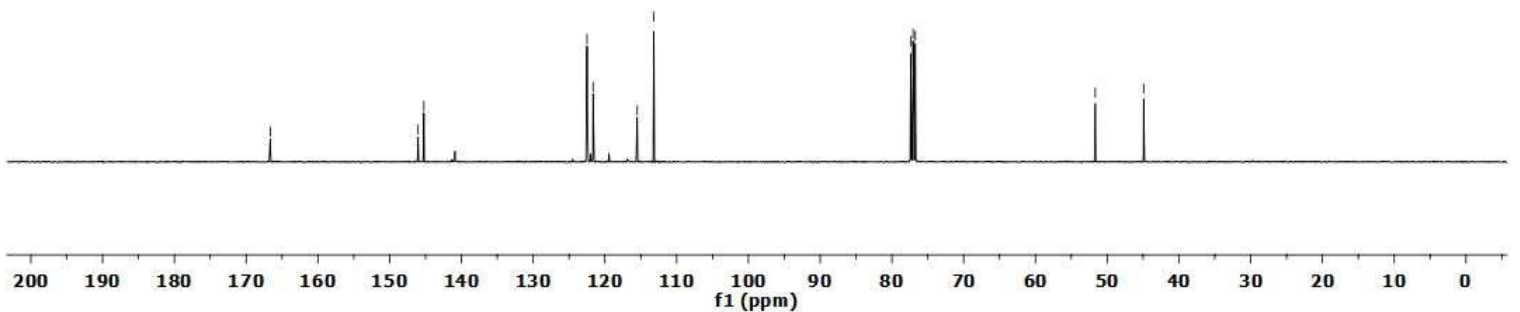

MSR_61D_225

26SEP2019_7 16 (0.310) AM2 (AS,22000.0,0.00,0.00): ABs; Cm (13:19-11:11+21:65)

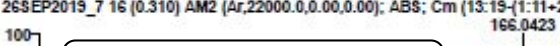

100

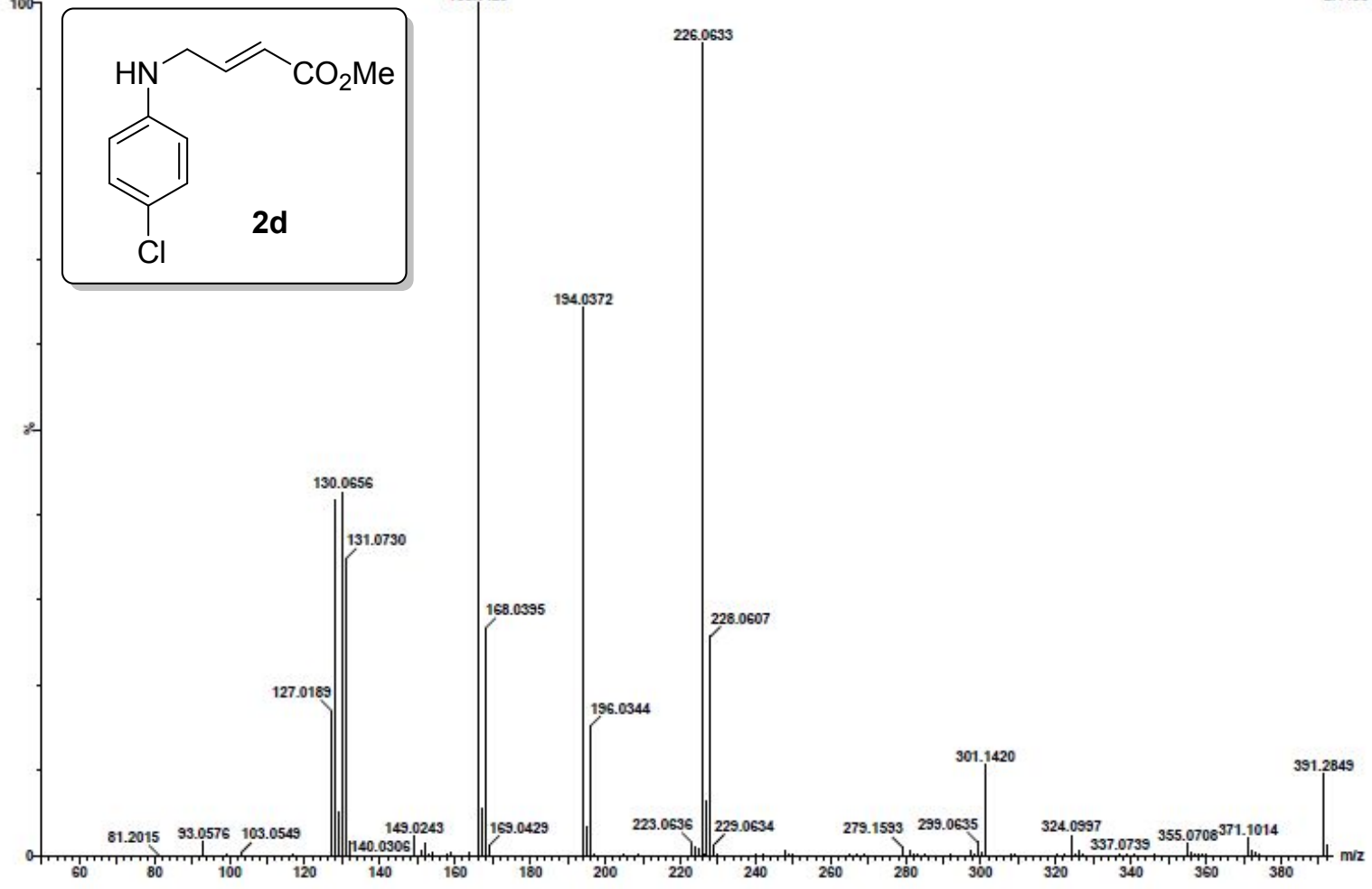

1: TOF MS ES- 


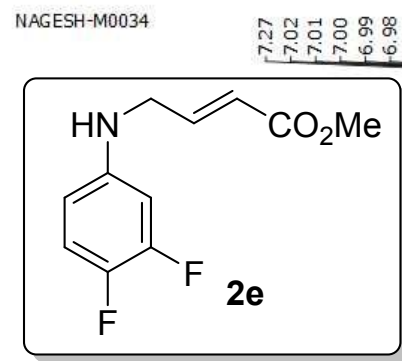

${ }^{1} \mathrm{H} \mathrm{NMR}\left(\mathrm{CDCl}_{3}, 400 \mathrm{MHz}\right)$

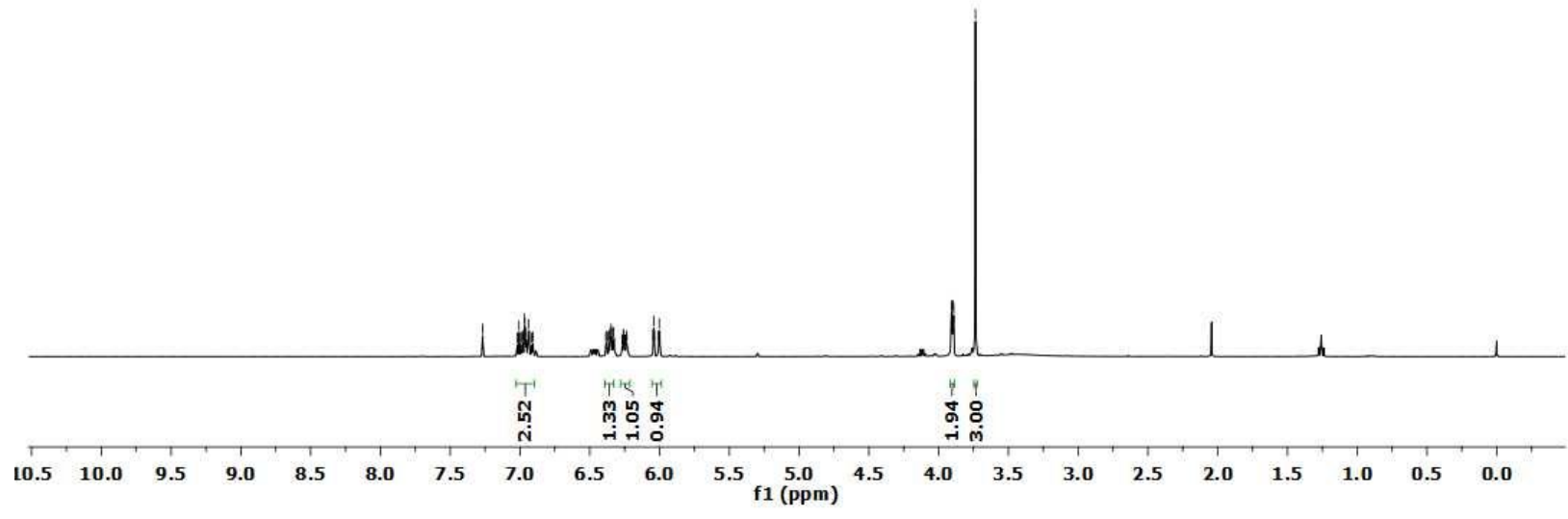

DATTAHARI-H0011

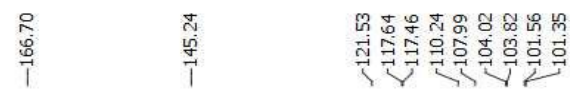

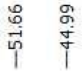

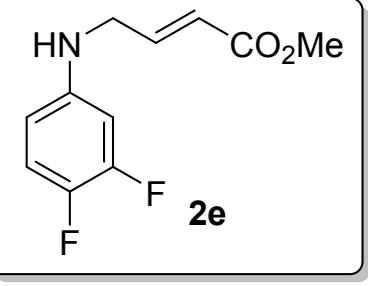

${ }^{13} \mathrm{C} \mathrm{NMR}\left(\mathrm{CDCl}_{3}, 100 \mathrm{MHz}\right)$

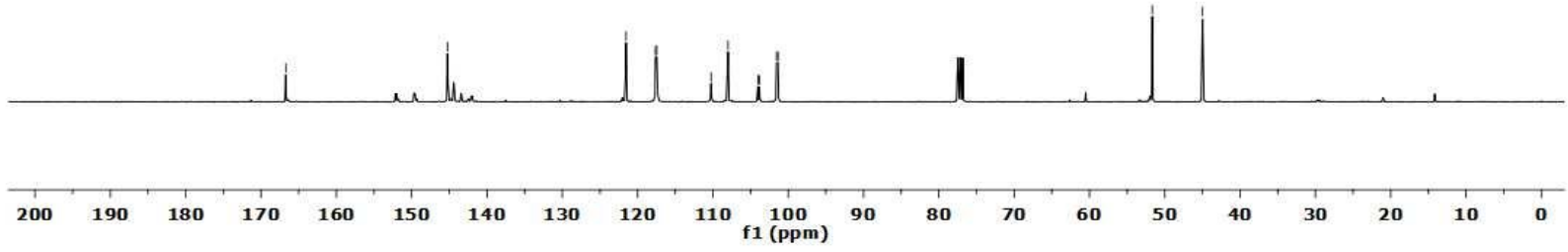




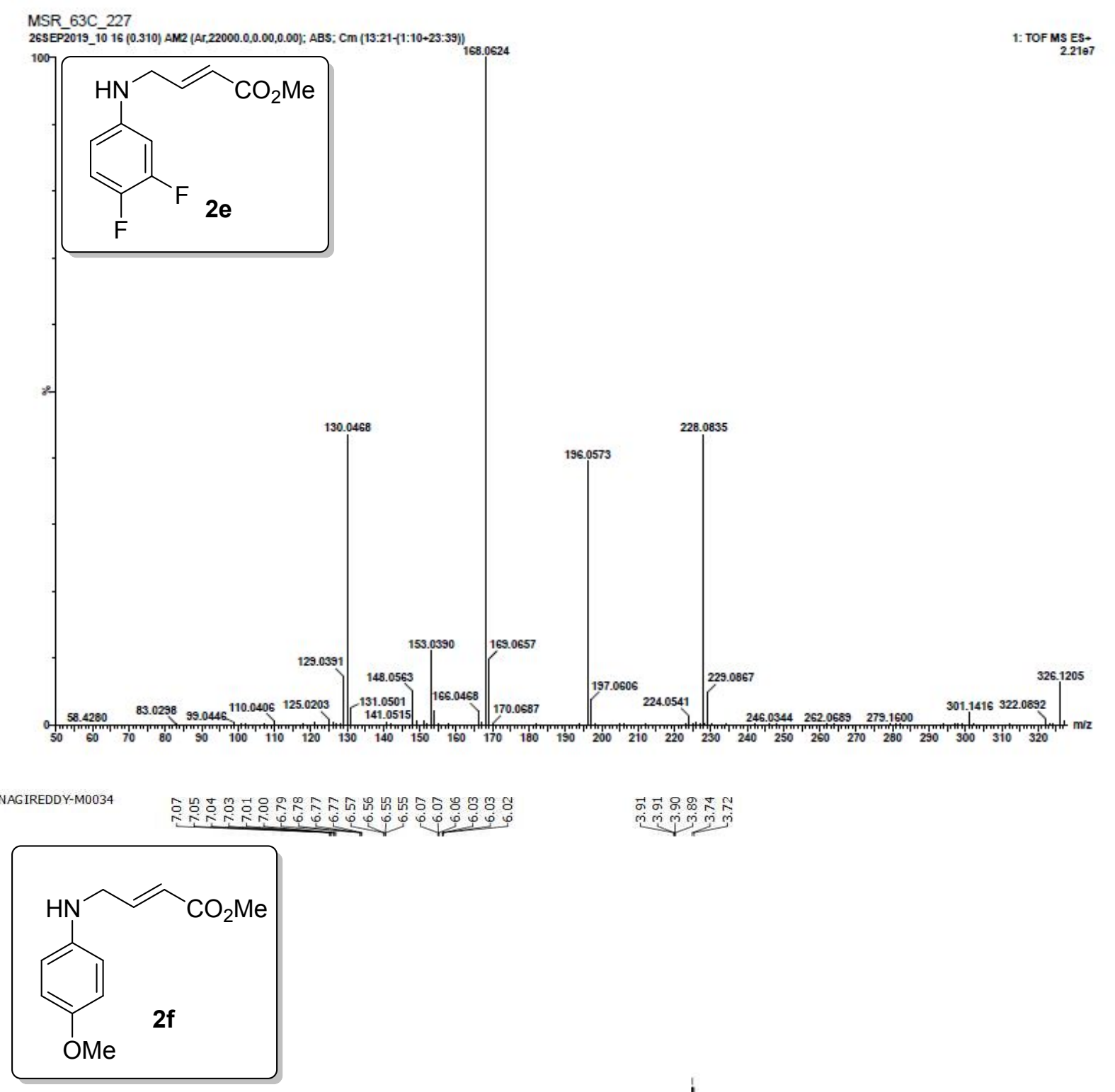

${ }^{1} \mathrm{H} \mathrm{NMR}\left(\mathrm{CDCl}_{3}, 500 \mathrm{MHz}\right)$

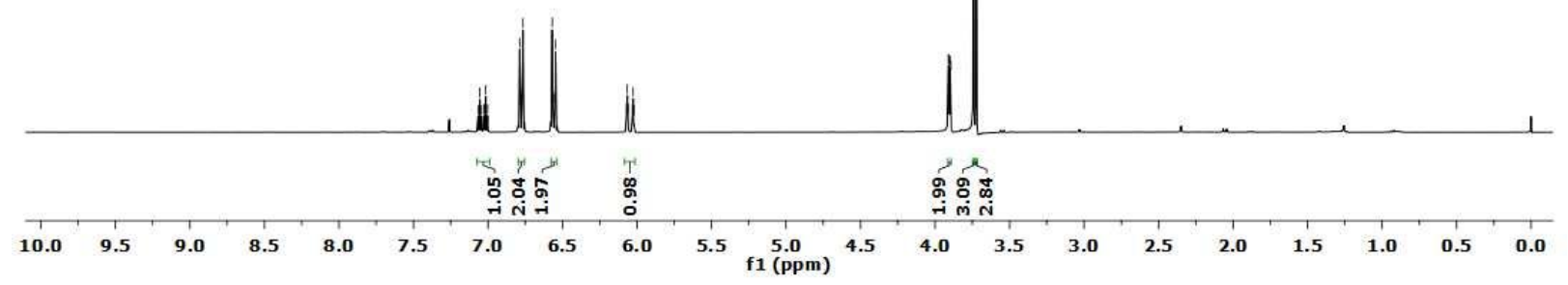


MANEESH-M0034

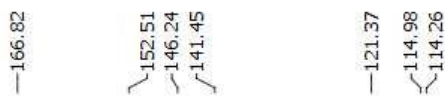

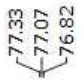

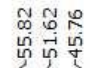

竝

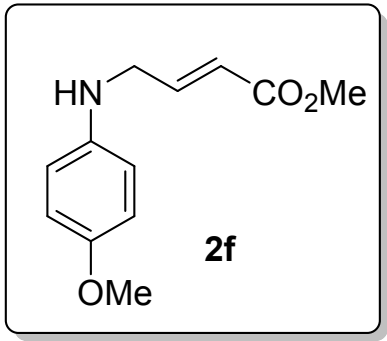

${ }^{13} \mathrm{C} \mathrm{NMR}\left(\mathrm{CDCl}_{3}, 125 \mathrm{MHz}\right)$
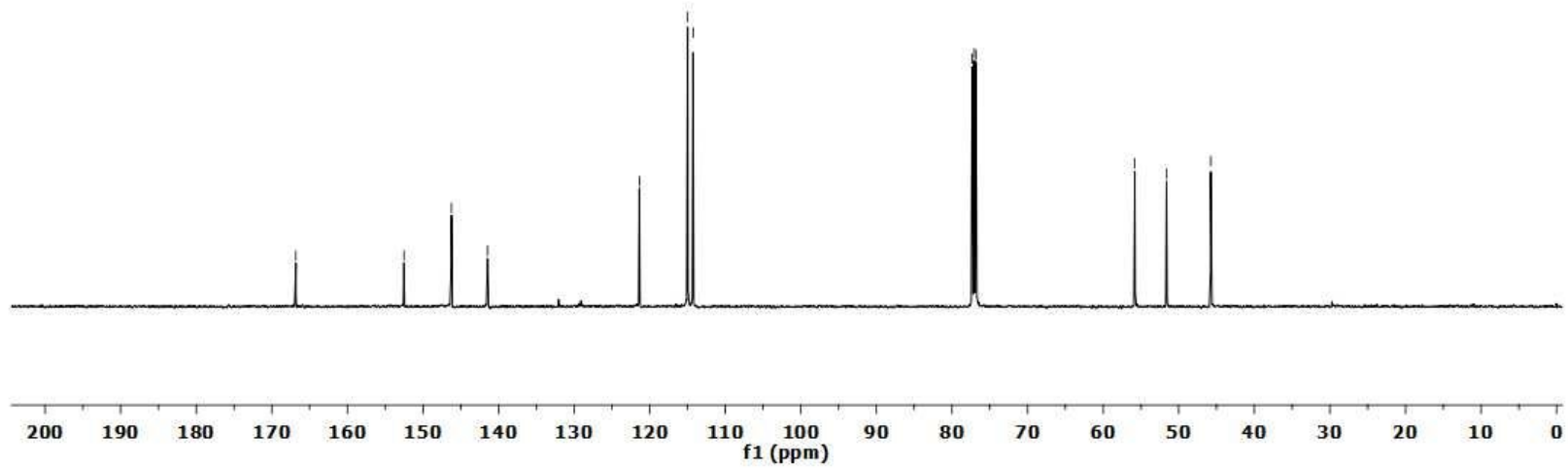

MSR_108B_221

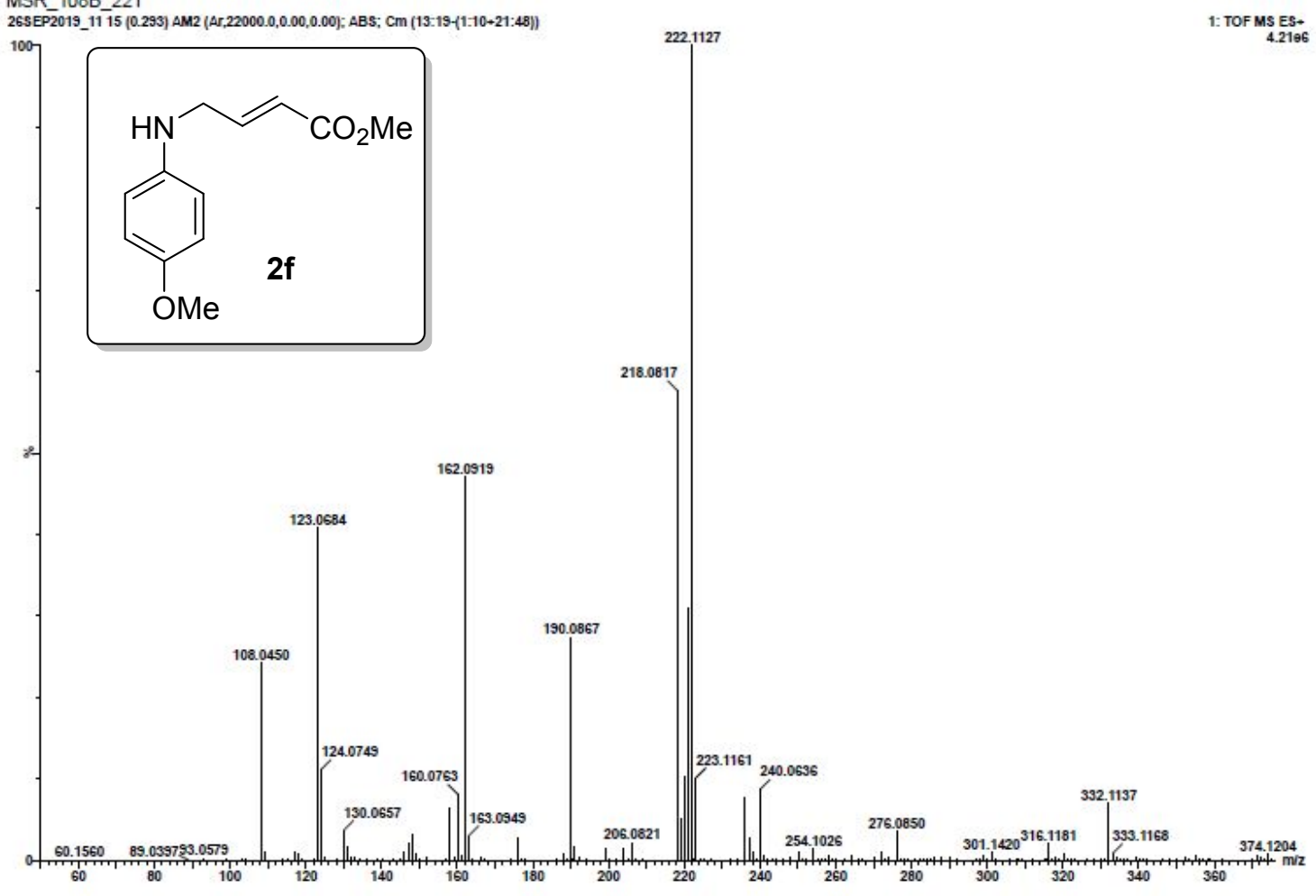




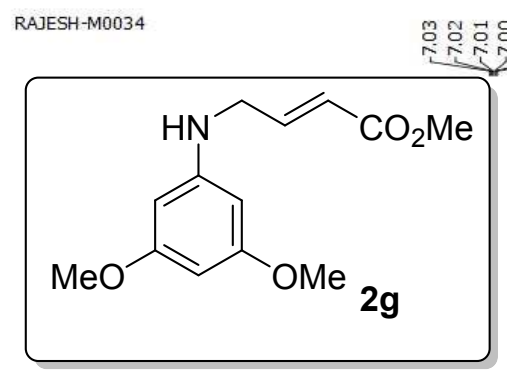

${ }^{1} \mathrm{H} \mathrm{NMR}\left(\mathrm{CDCl}_{3}, 500 \mathrm{MHz}\right)$
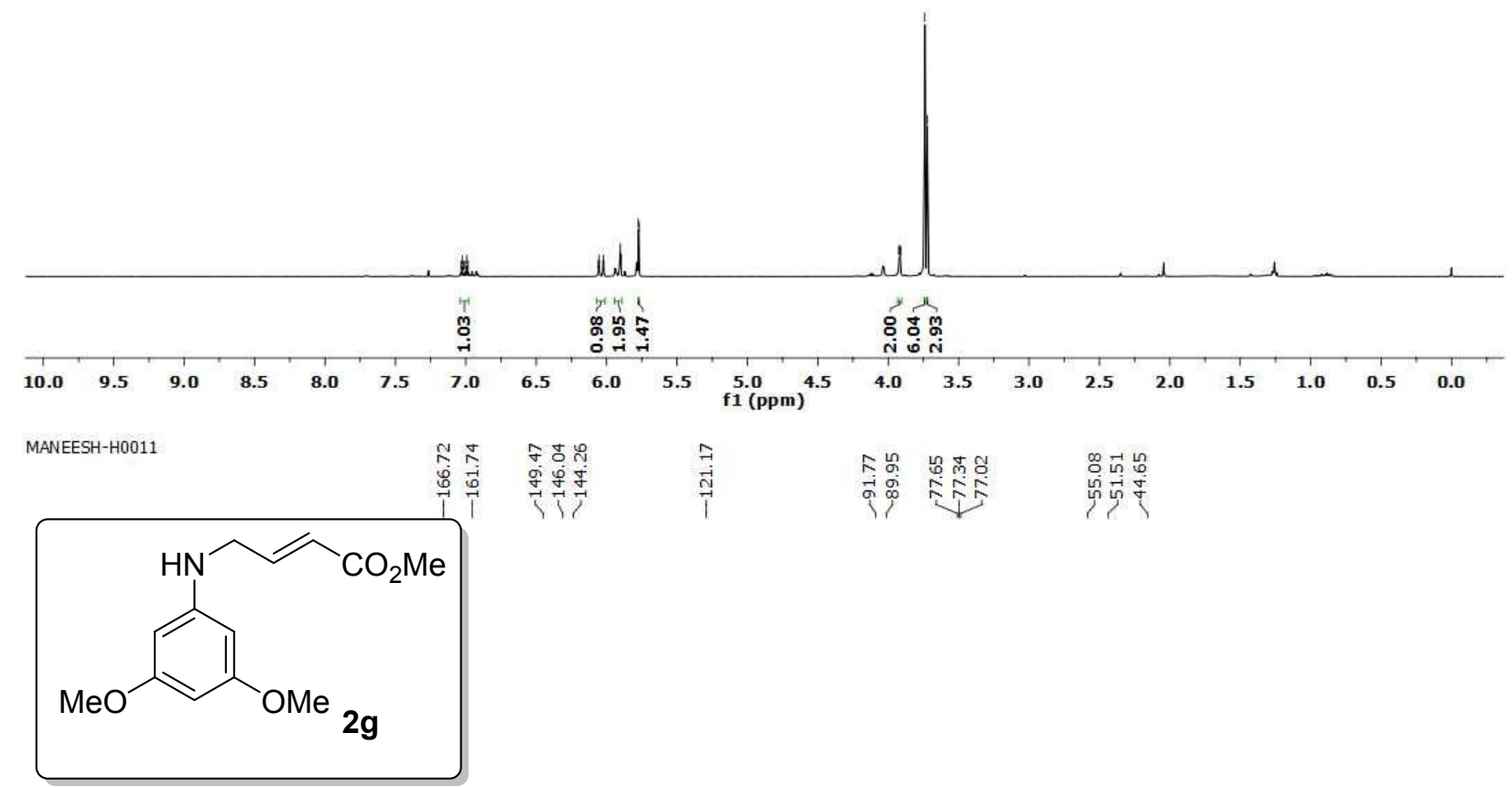

${ }^{13} \mathrm{C} \mathrm{NMR}\left(\mathrm{CDCl}_{3}, 125 \mathrm{MHz}\right)$

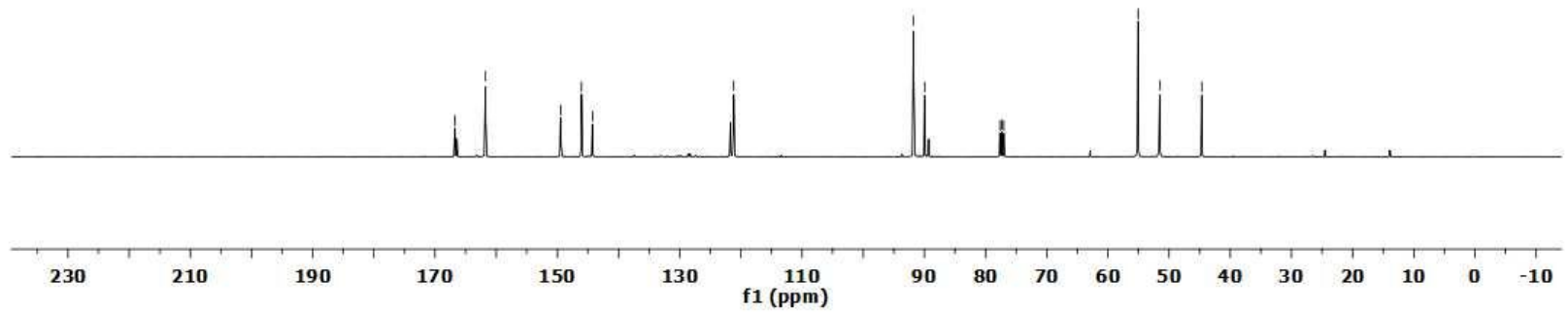




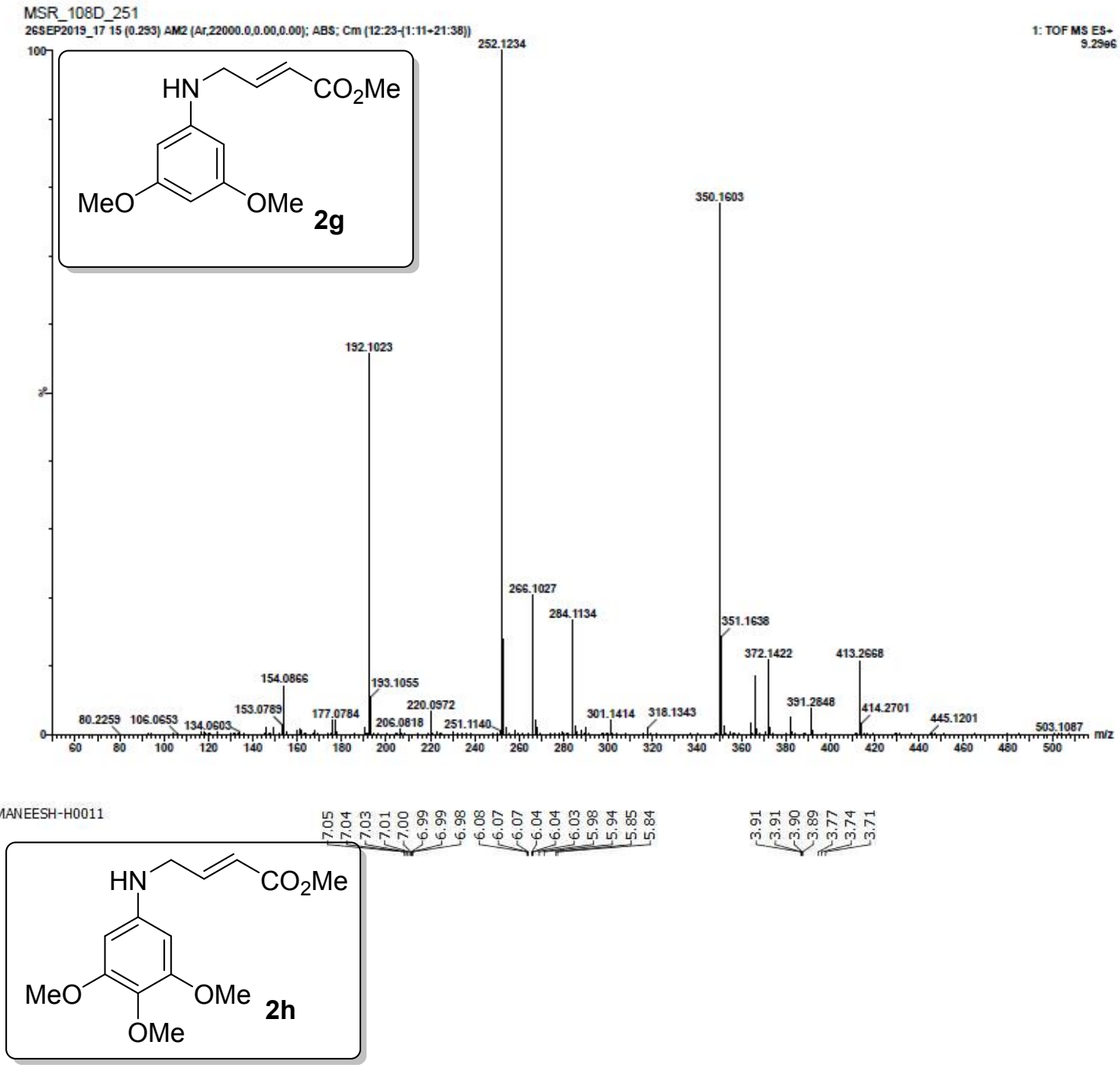

${ }^{1} \mathrm{H} \mathrm{NMR}\left(\mathrm{CDCl}_{3}, 400 \mathrm{MHz}\right)$

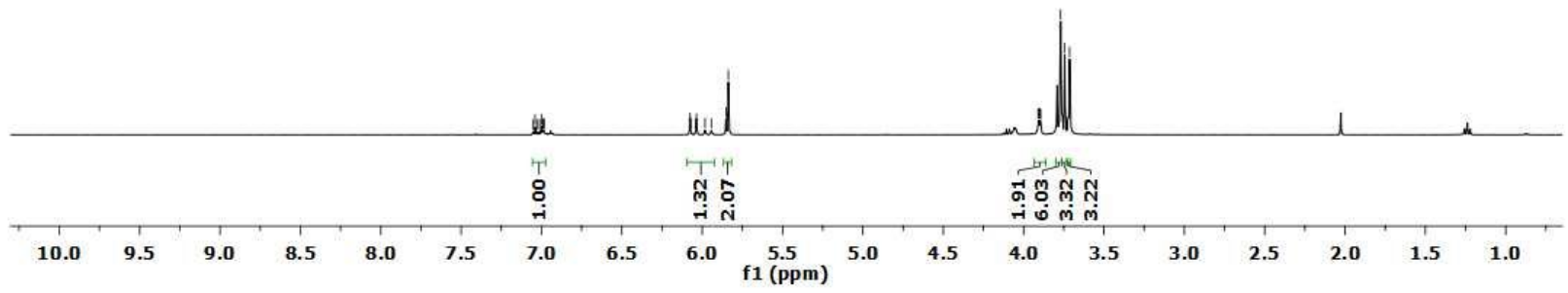




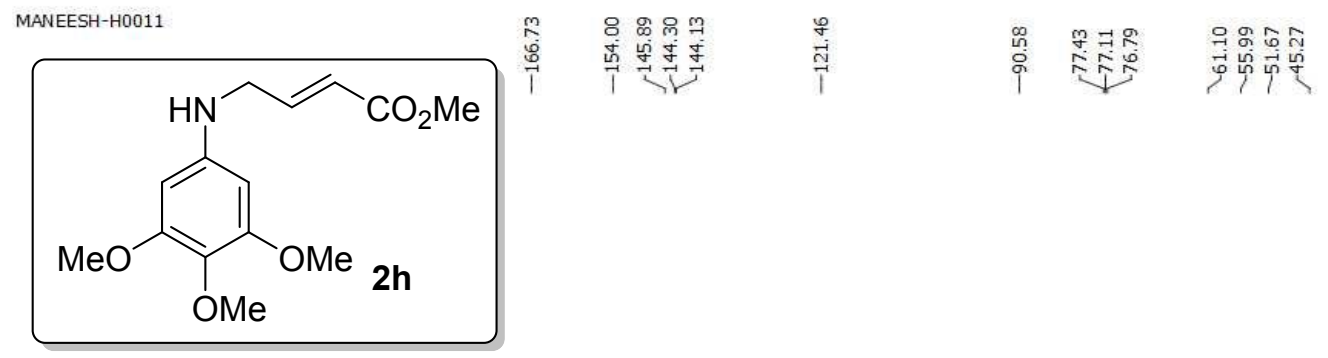

${ }^{13} \mathrm{C} \mathrm{NMR}\left(\mathrm{CDCl}_{3}, 100 \mathrm{MHz}\right)$
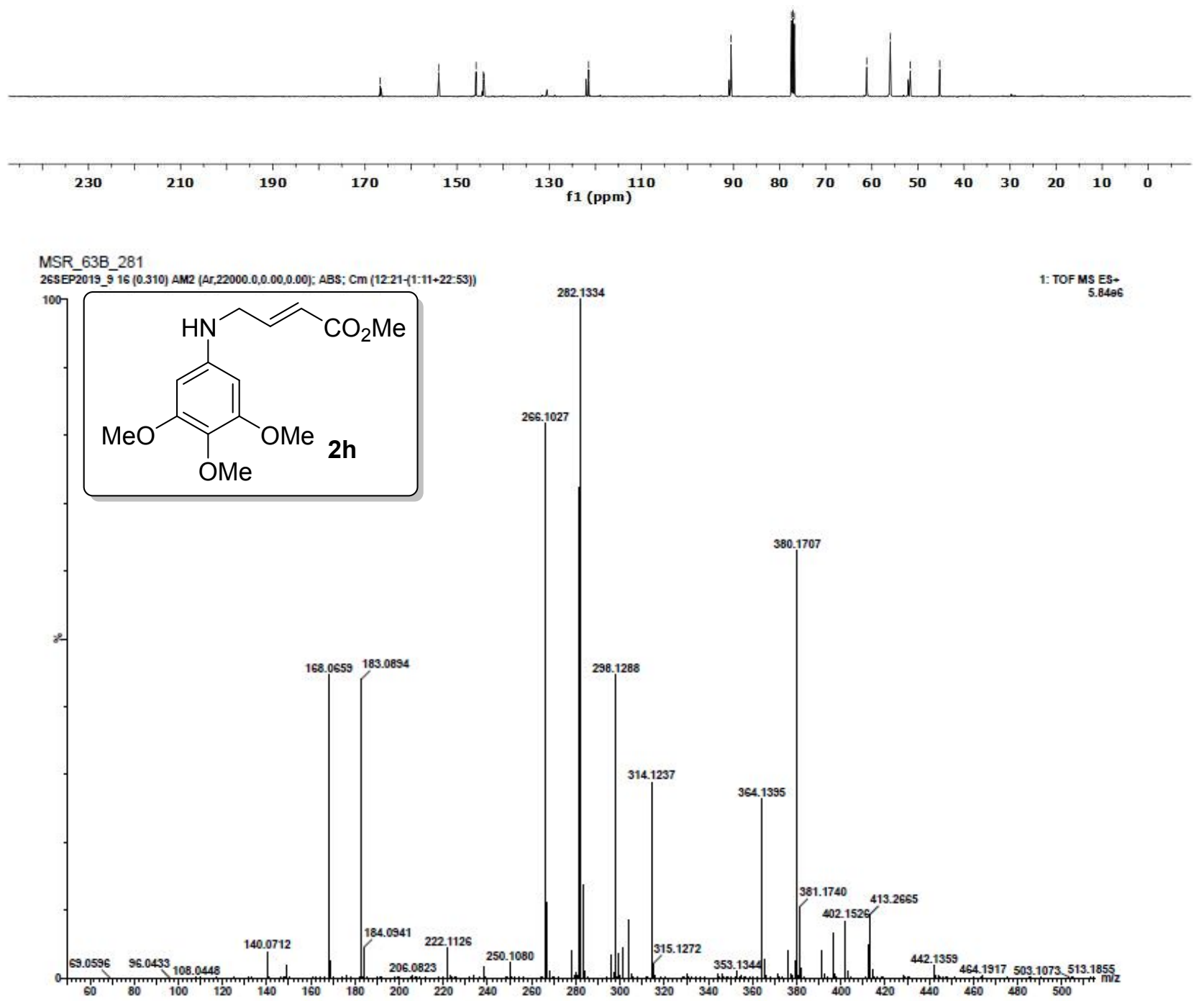

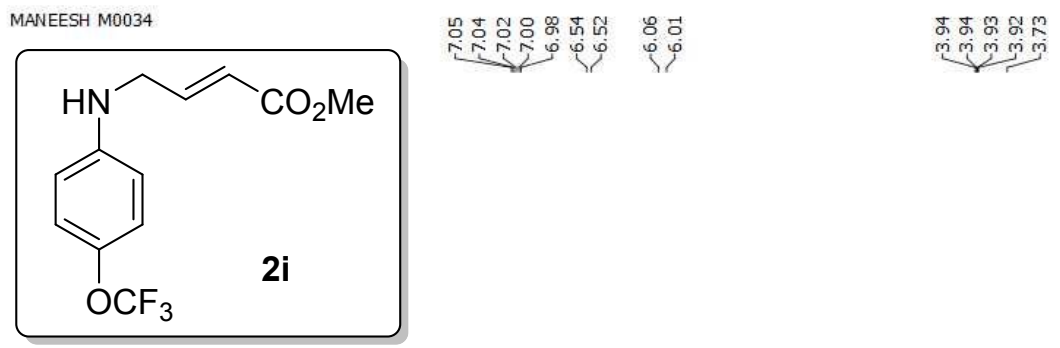

${ }^{1} \mathrm{H}$ NMR $\left(\mathrm{CDCl}_{3}, 400 \mathrm{MHz}\right)$
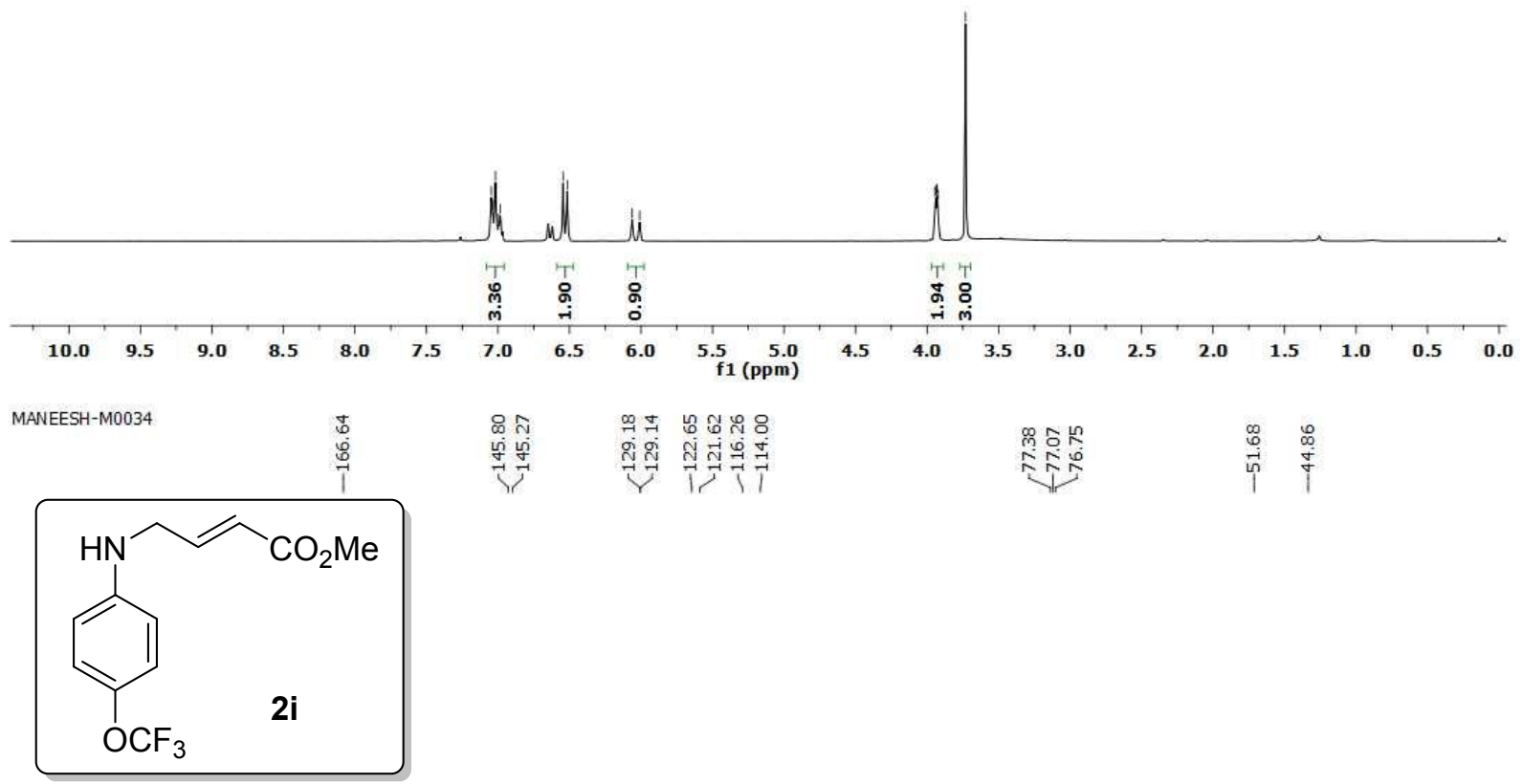

${ }^{13} \mathrm{C} \mathrm{NMR}\left(\mathrm{CDCl}_{3}, 100 \mathrm{MHz}\right)$

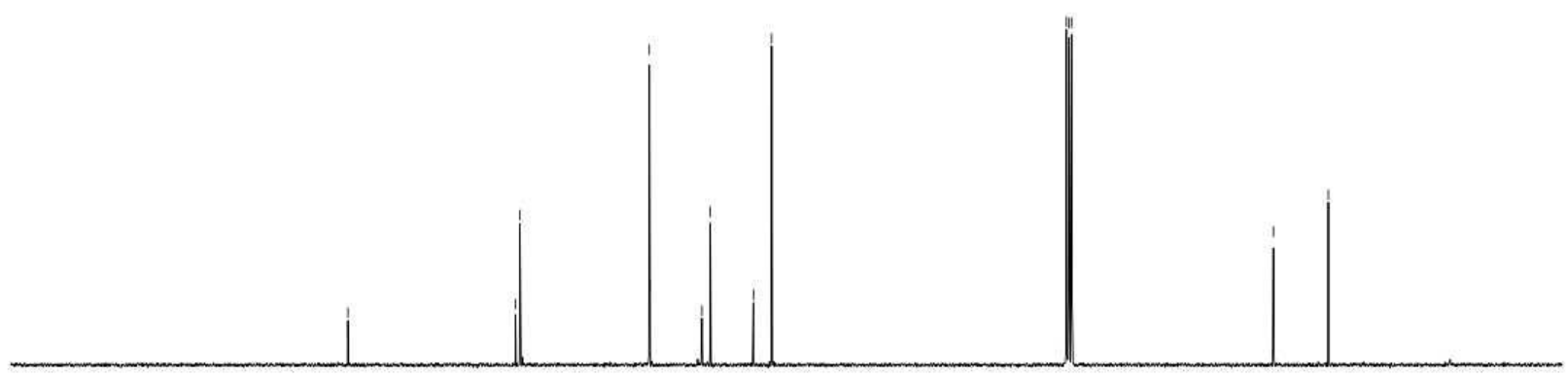




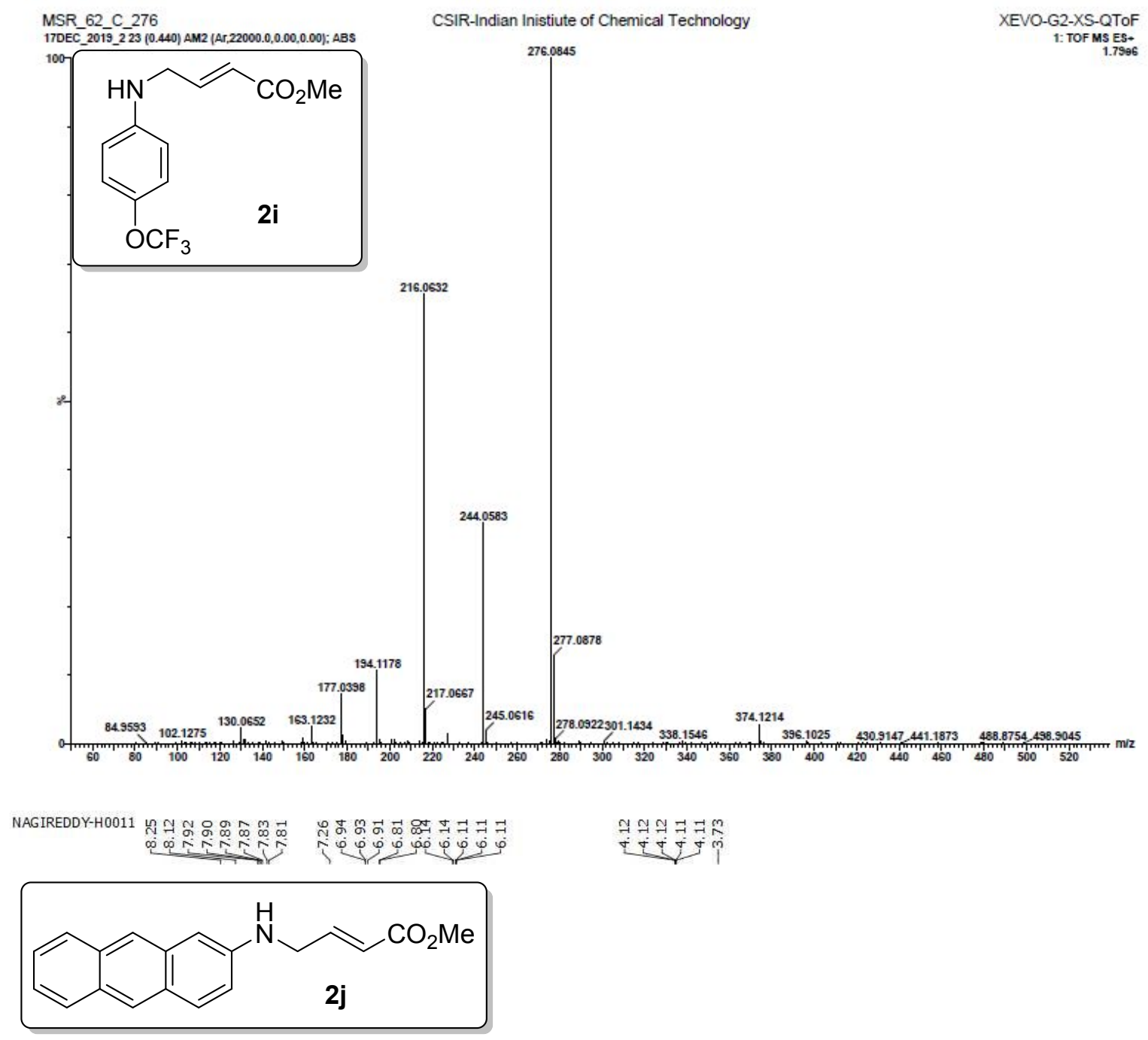

${ }^{1} \mathrm{H}$ NMR $\left(\mathrm{CDCl}_{3}, 500 \mathrm{MHz}\right)$

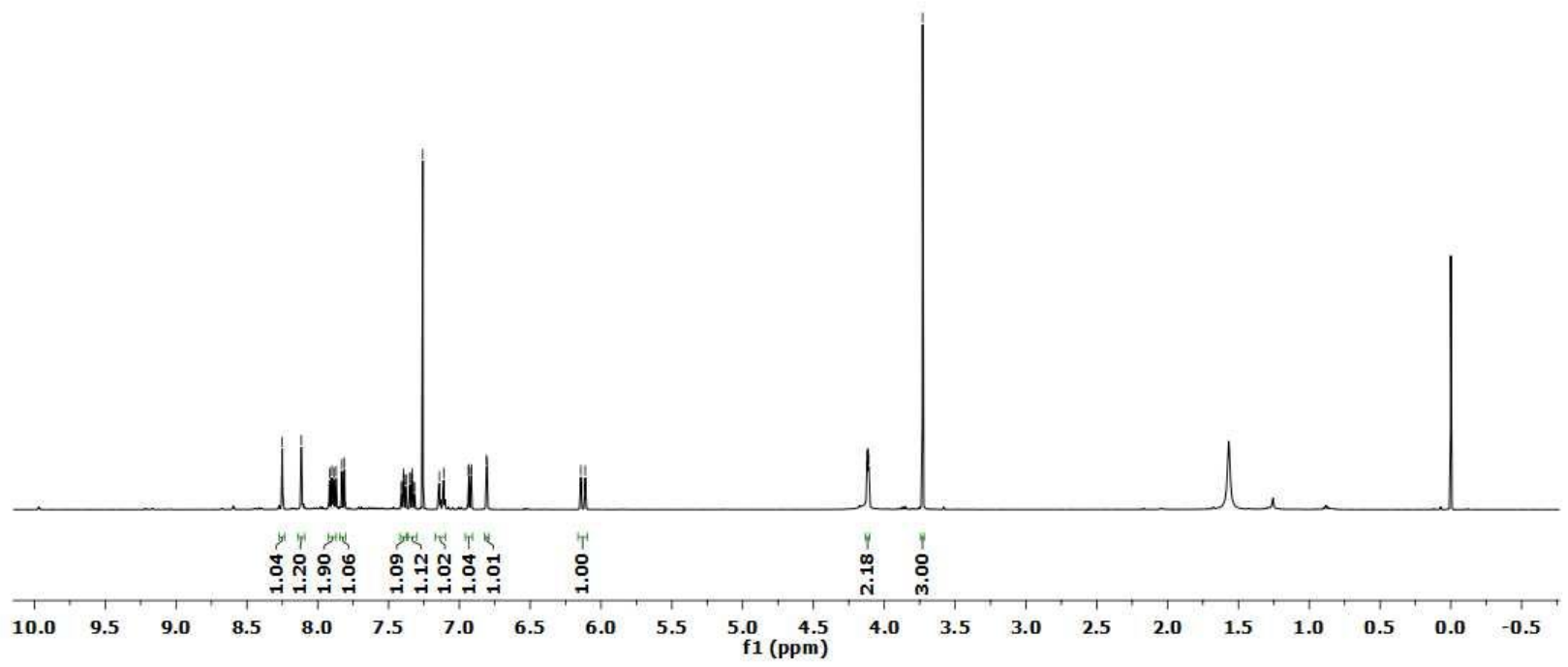




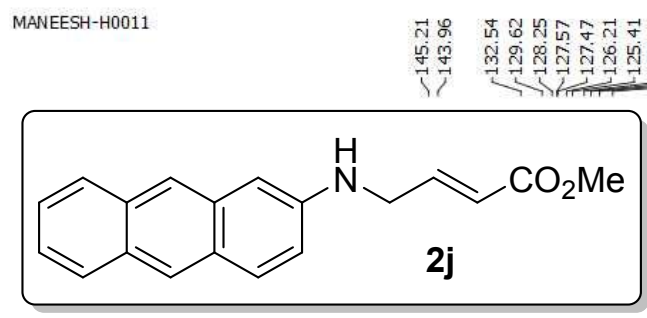

${ }^{13} \mathrm{C} \mathrm{NMR}\left(\mathrm{CDCl}_{3}, 125 \mathrm{MHz}\right)$
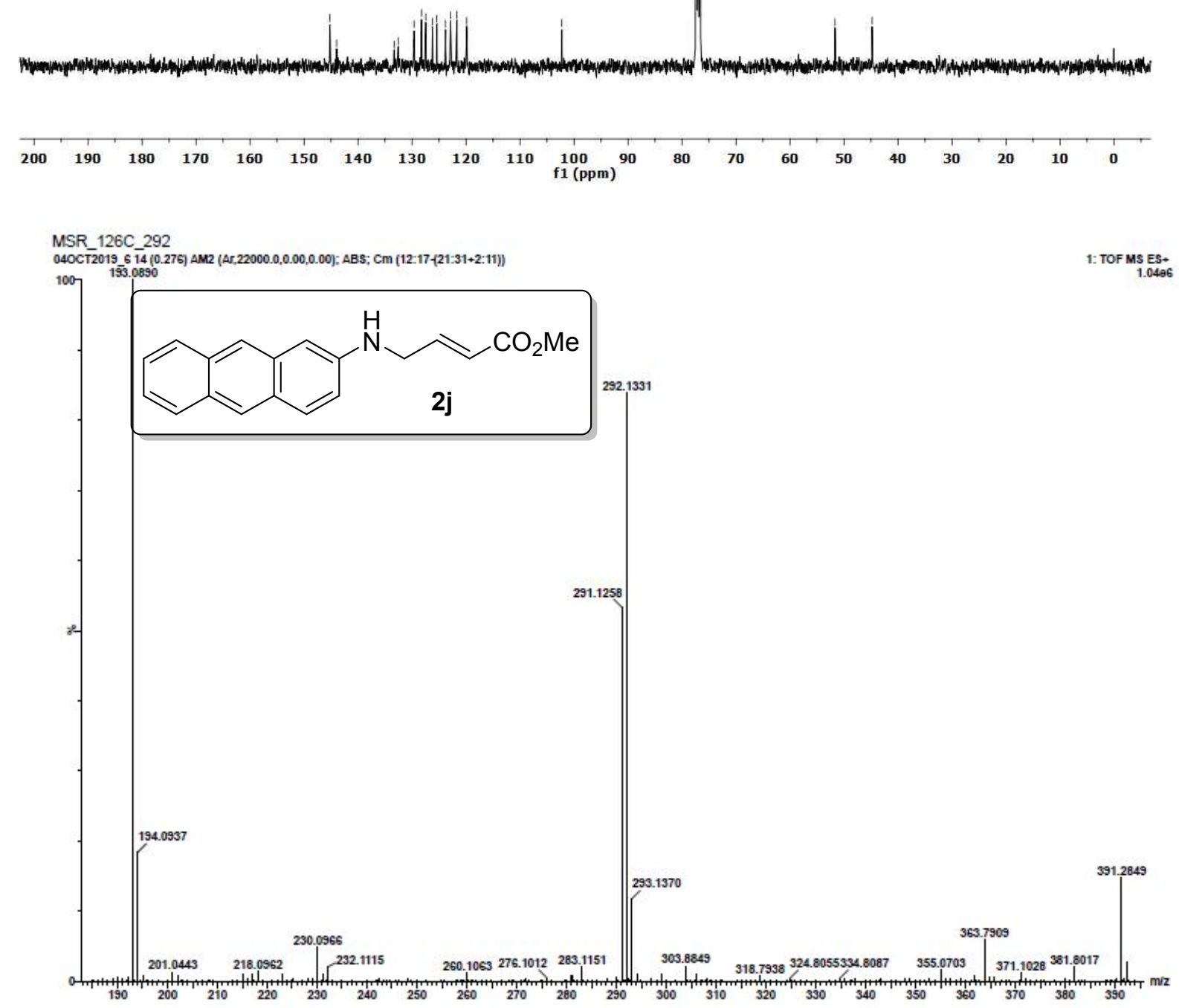

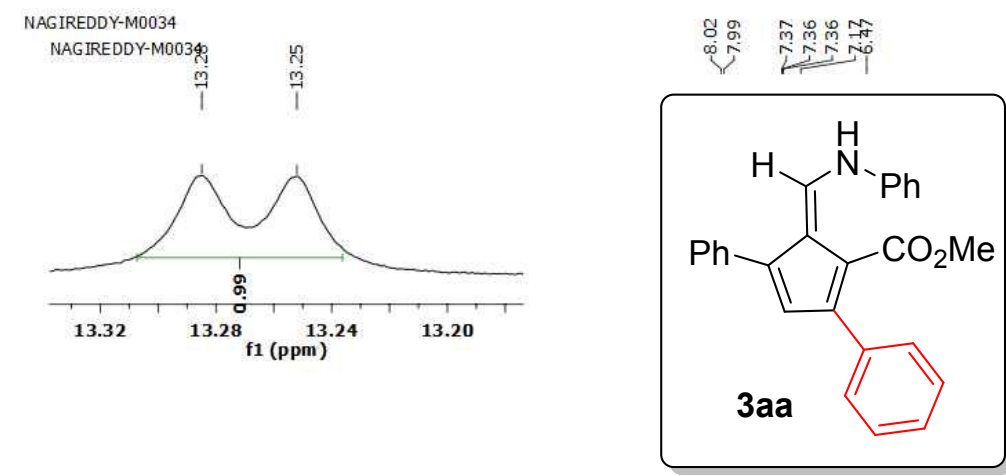

${ }^{1} \mathrm{H} \mathrm{NMR}\left(\mathrm{CDCl}_{3}, 400 \mathrm{MHz}\right)$
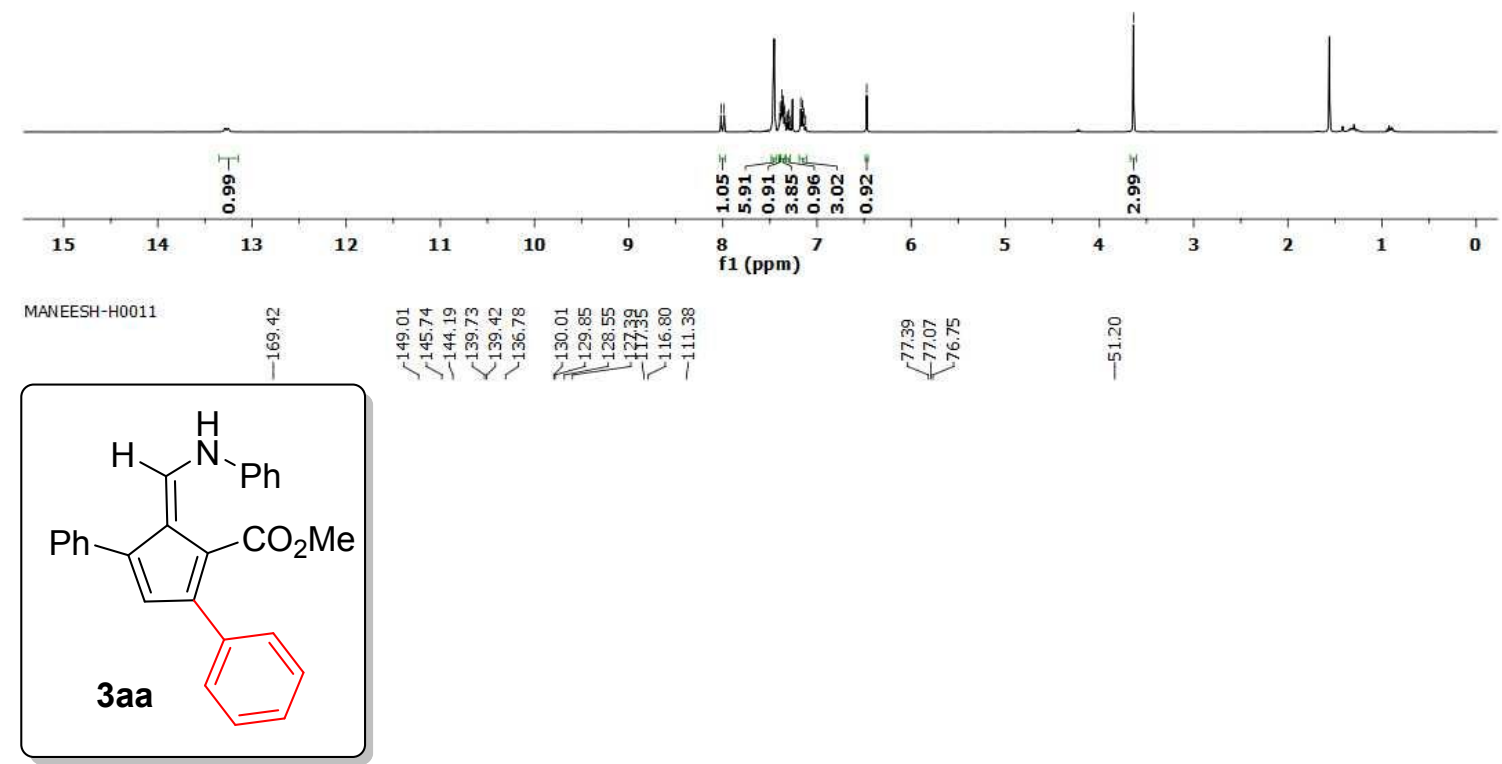

${ }^{13} \mathrm{C} \mathrm{NMR}\left(\mathrm{CDCl}_{3}, 100 \mathrm{MHz}\right)$
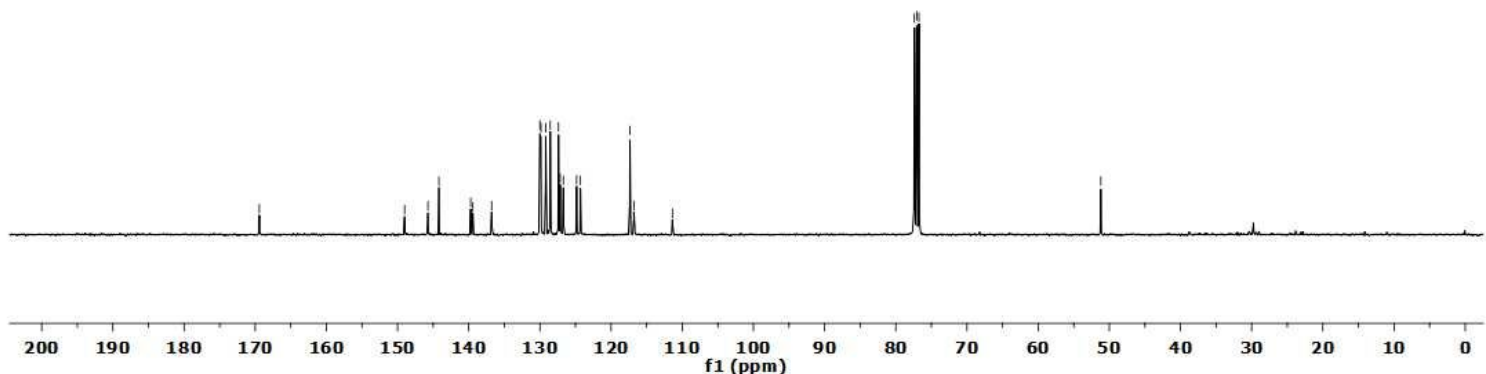


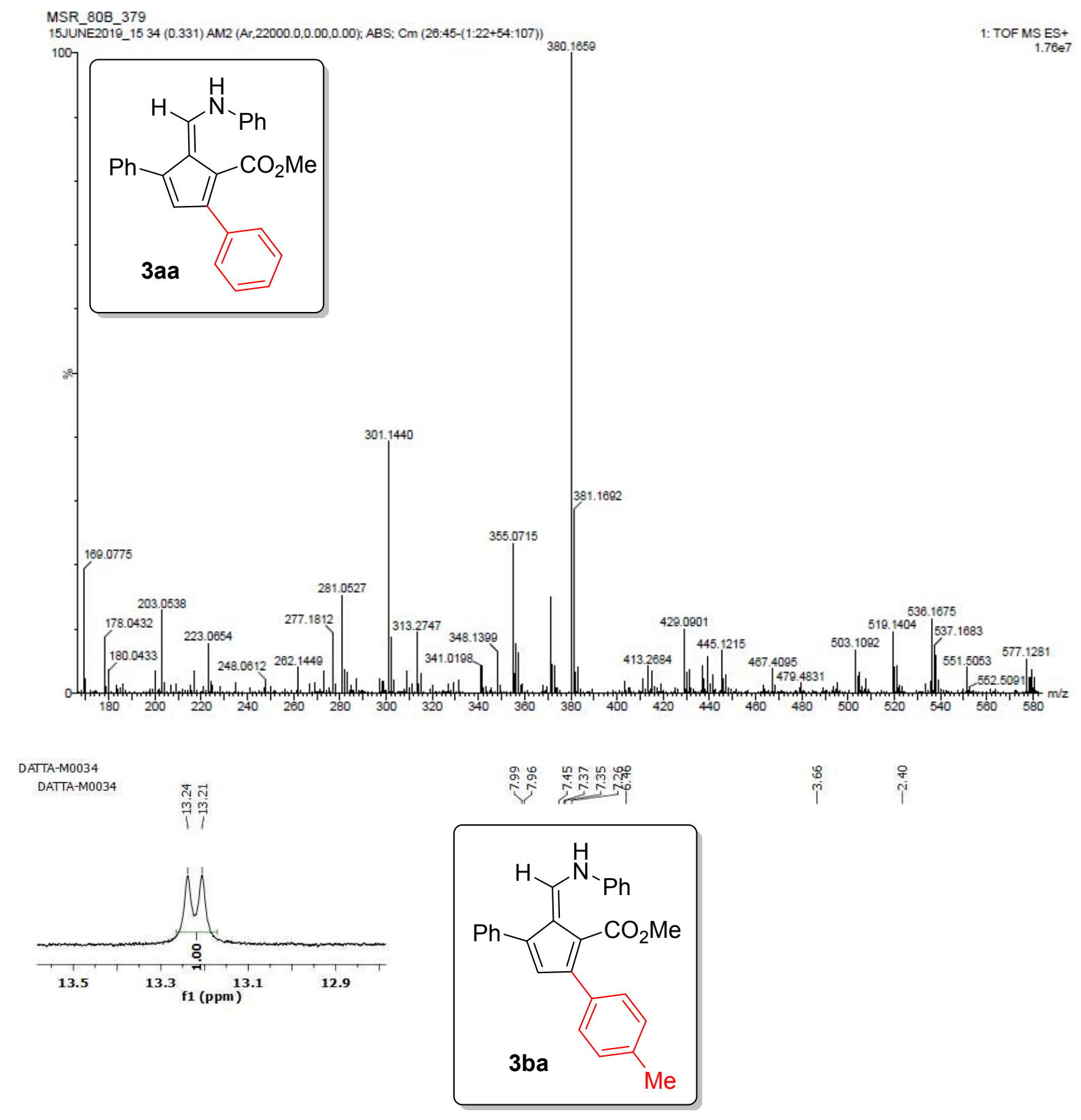

${ }^{1} \mathrm{H}$ NMR $\left(\mathrm{CDCl}_{3}, 400 \mathrm{MHz}\right)$

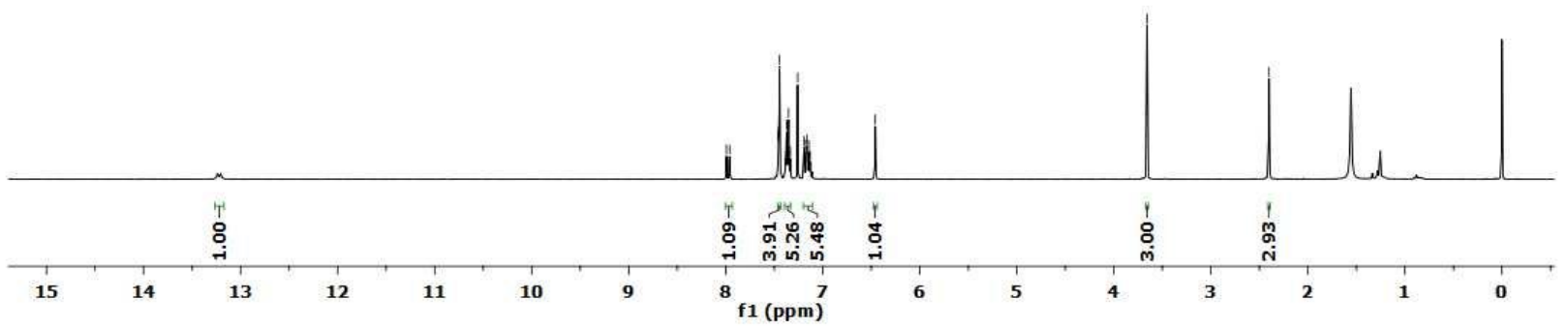



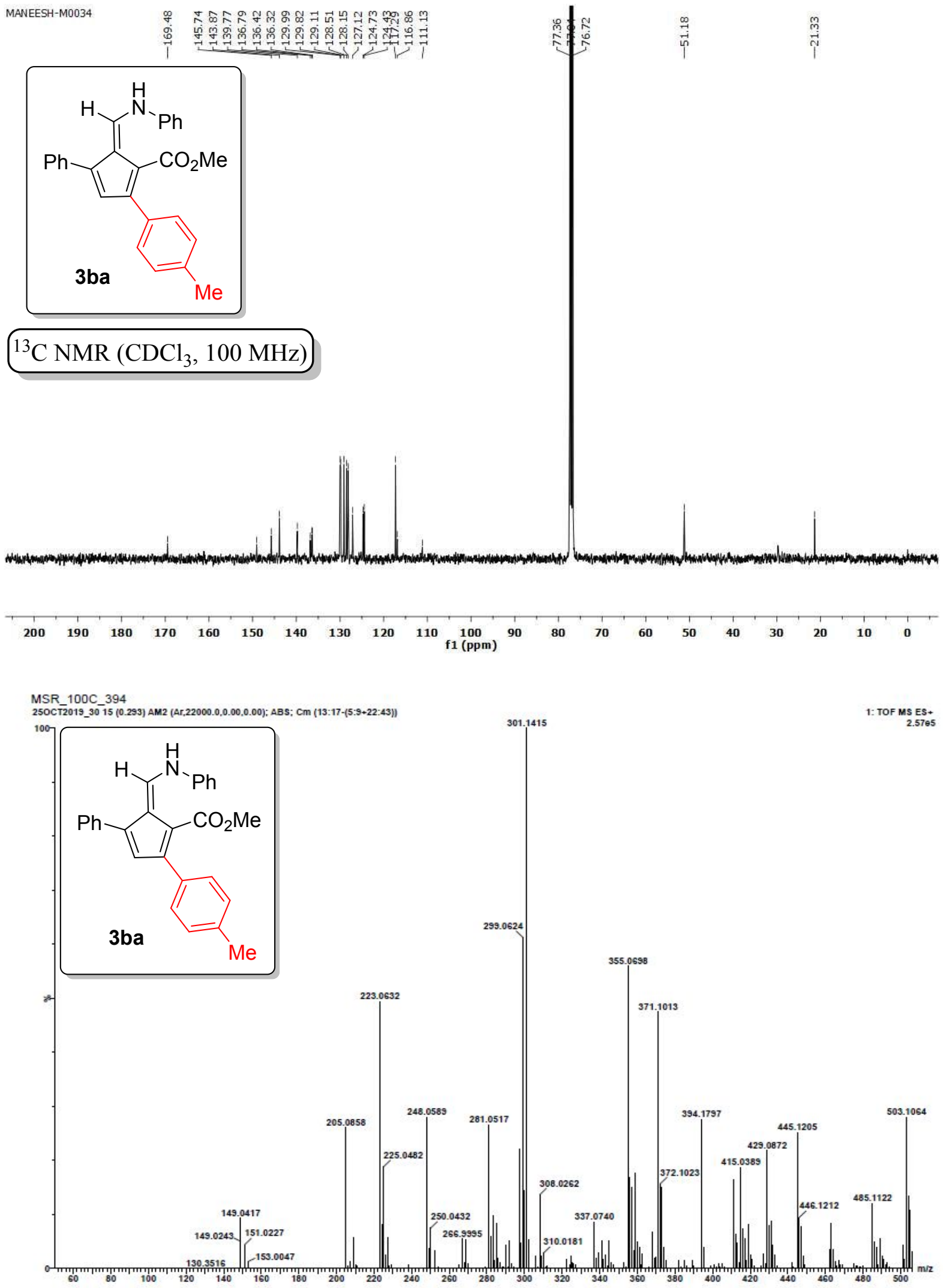


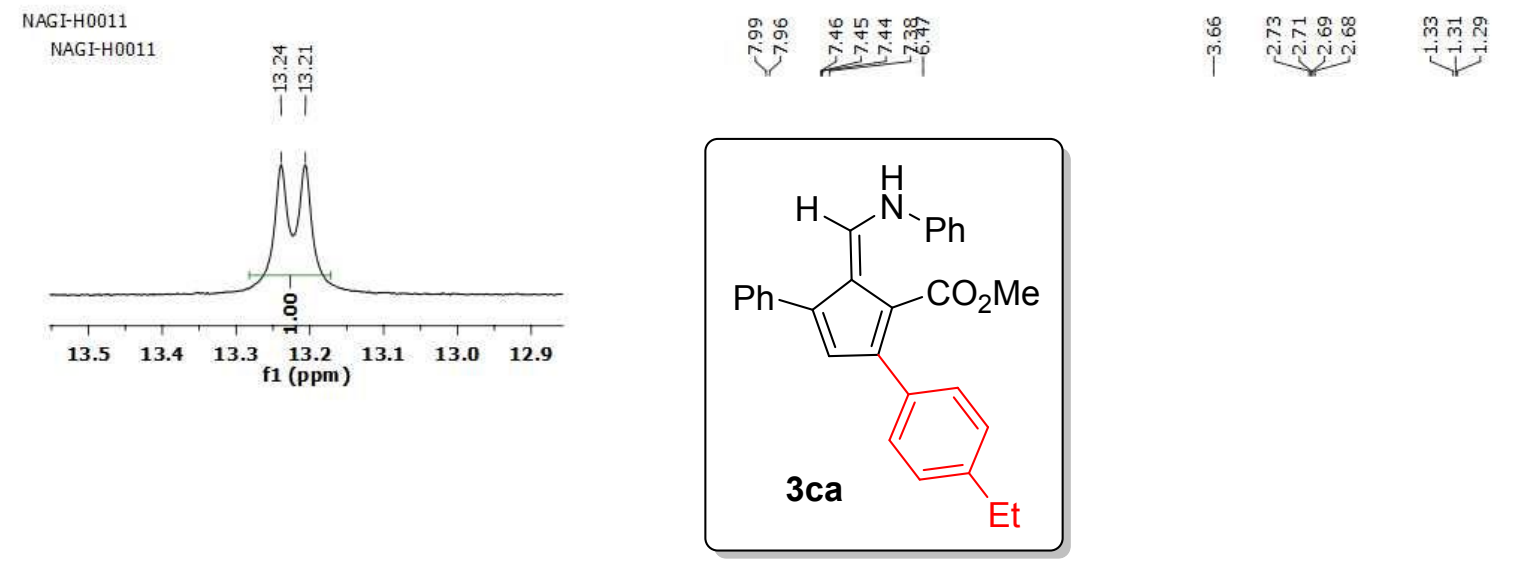

${ }^{1} \mathrm{H} \mathrm{NMR}\left(\mathrm{CDCl}_{3}, 400 \mathrm{MHz}\right)$

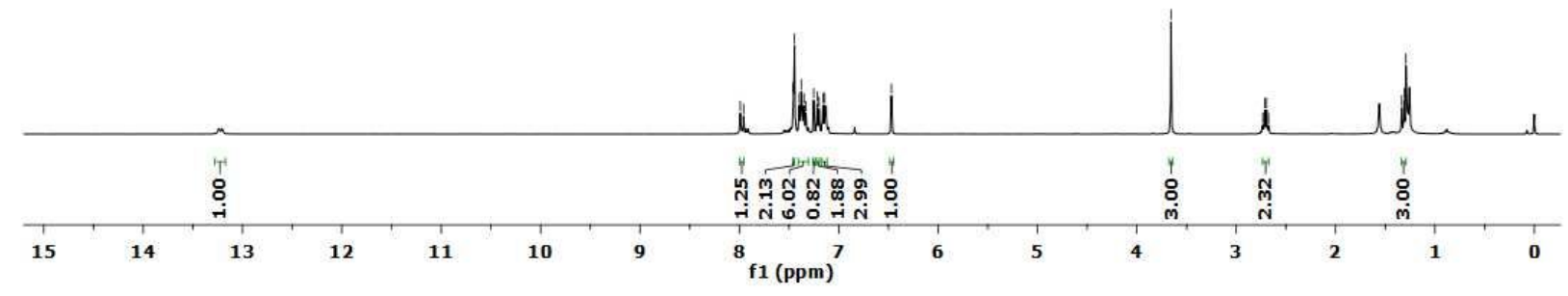

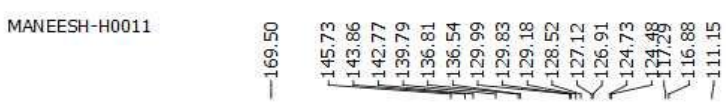
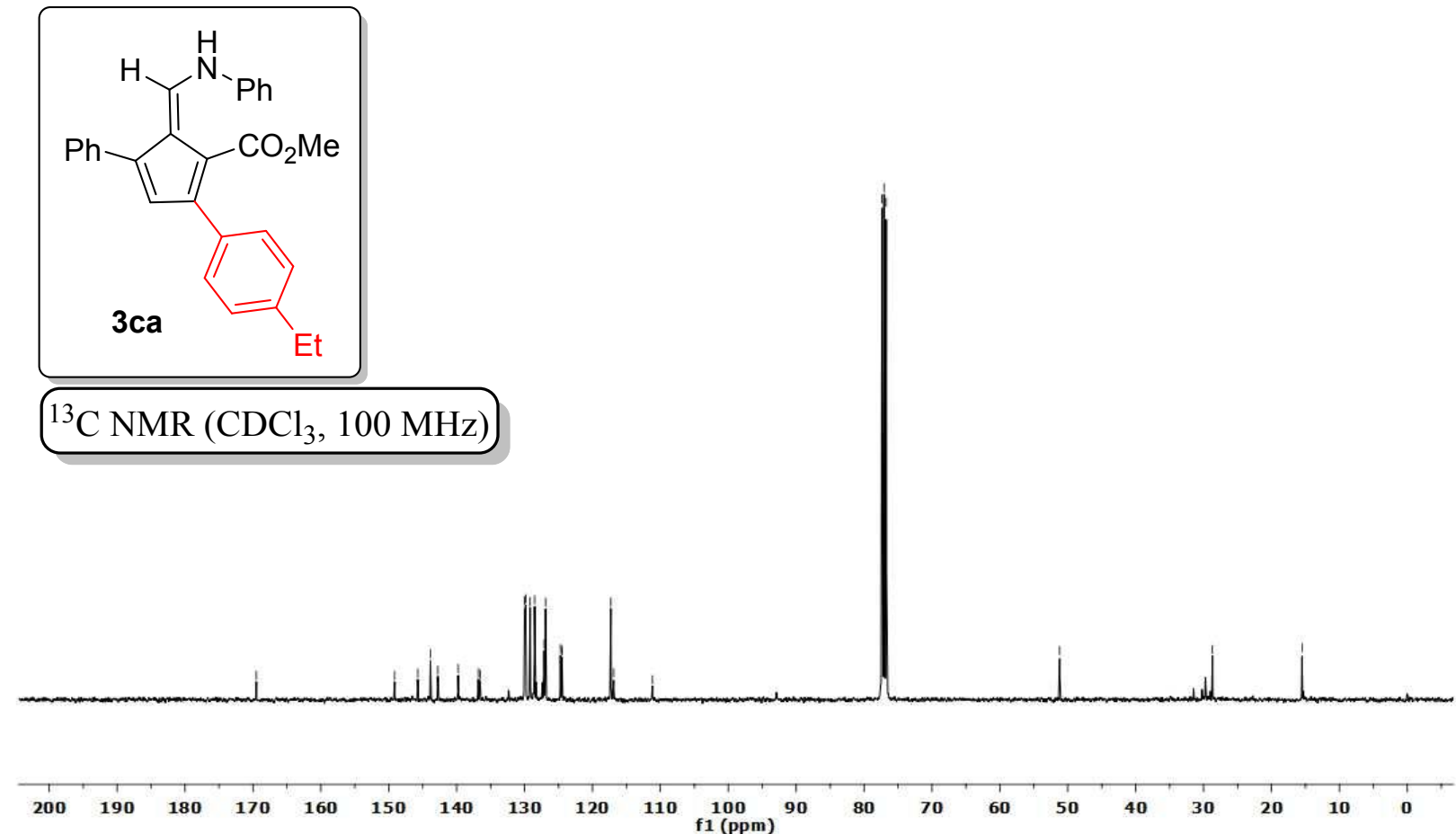
Maddi SRIDHAR REDDY

11/27/2019 3:55:44 PM

ThermoScientific EXACTIVE ORBITRAP

Analysed By G Saikrishna

MSR-107-B \#1-13 ${ }^{-} \mathrm{RT}^{-} \mathrm{C}^{-} 0.01-0.05^{-} \mathrm{AV}^{-}{ }^{-1}{ }^{-} \mathrm{NL}:{ }^{-2} 2.22 \mathrm{E} 8$

T: FTMS $\{1,1\}+p$ APCl corona Full ms [100.00-2000.00]

408.19616

$\mathrm{C}_{28} \mathrm{H}_{26} \mathrm{O}_{2} \mathrm{~N}=408.19581$ $1 0 0 \longdiv { N } \quad \begin{array} { c } { 1 6 . 5 \mathrm { RDBE } } \\ { 0 . 8 6 5 7 3 \mathrm { ppm } } \end{array}$

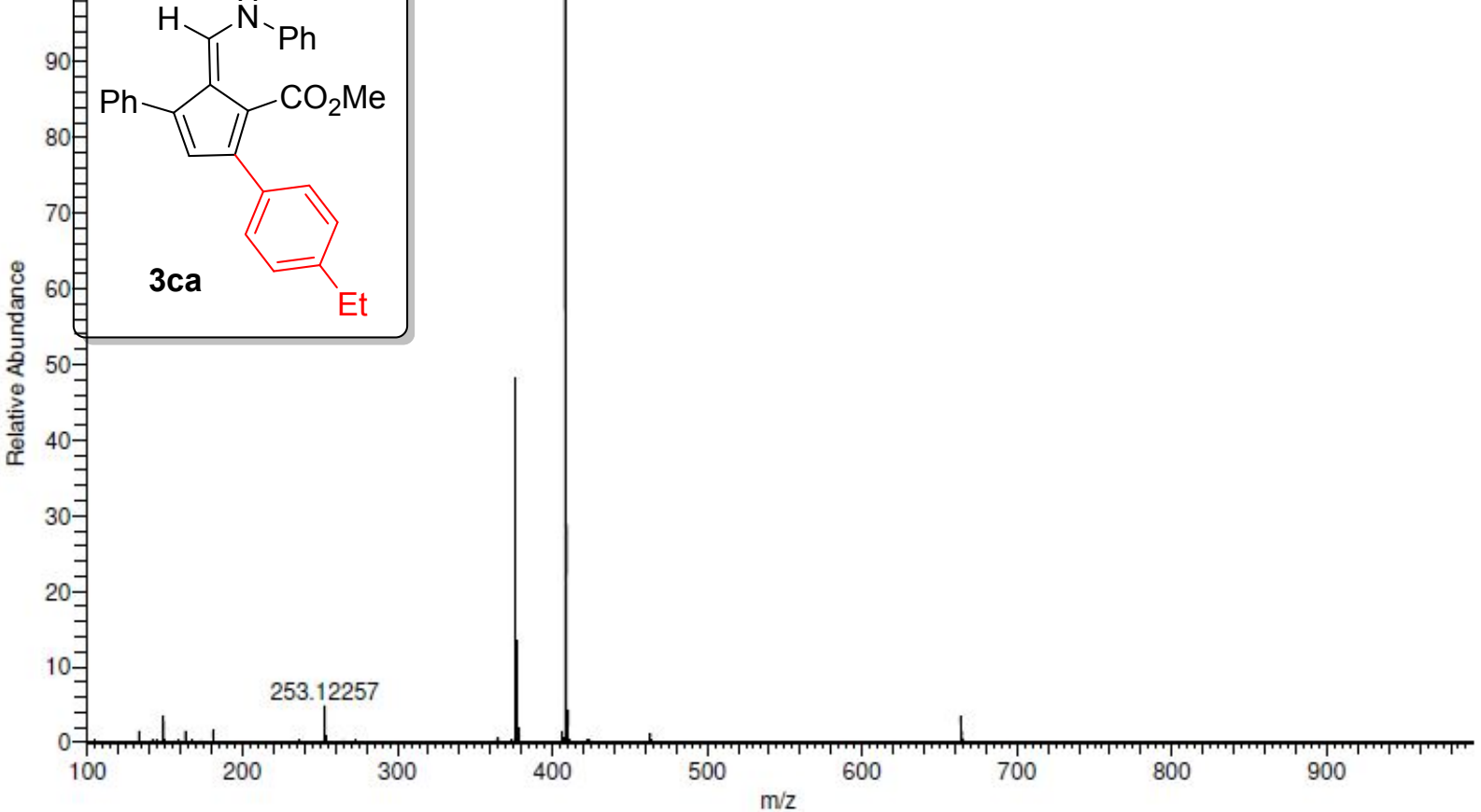

PARAM-M0034

PARAM-M0034

๙ั่

$\stackrel{\leftrightarrow}{i}$

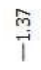
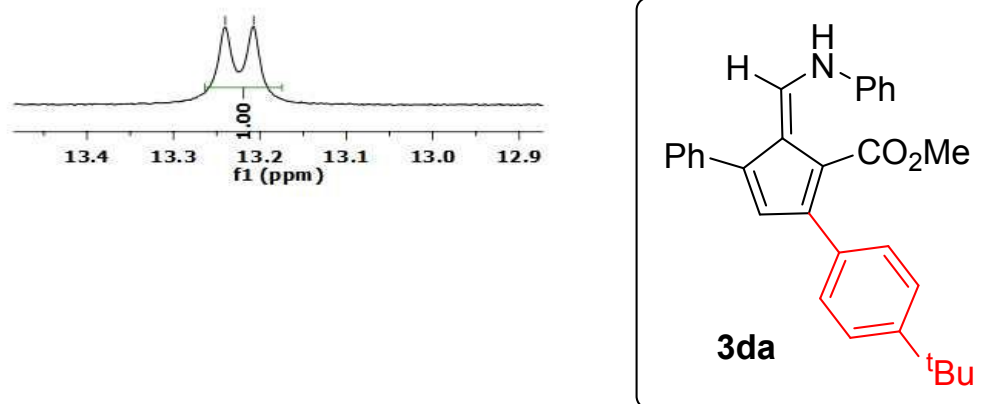

${ }^{1} \mathrm{H}$ NMR $\left(\mathrm{CDCl}_{3}, 400 \mathrm{MHz}\right)$

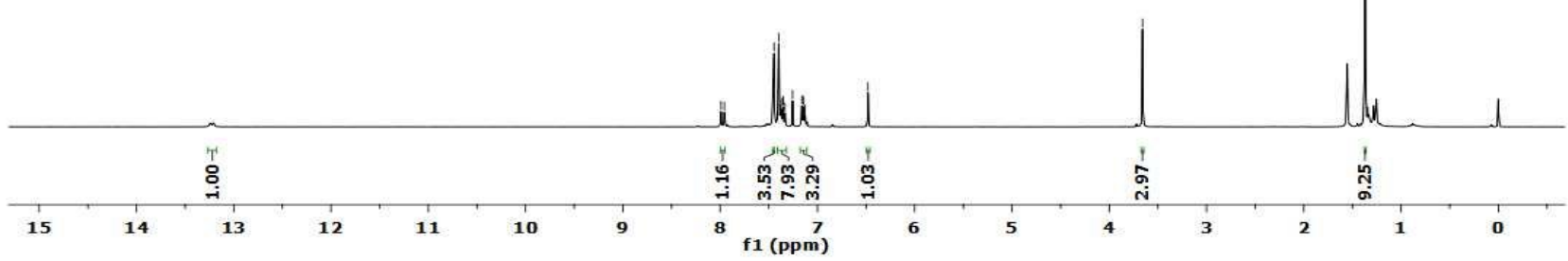



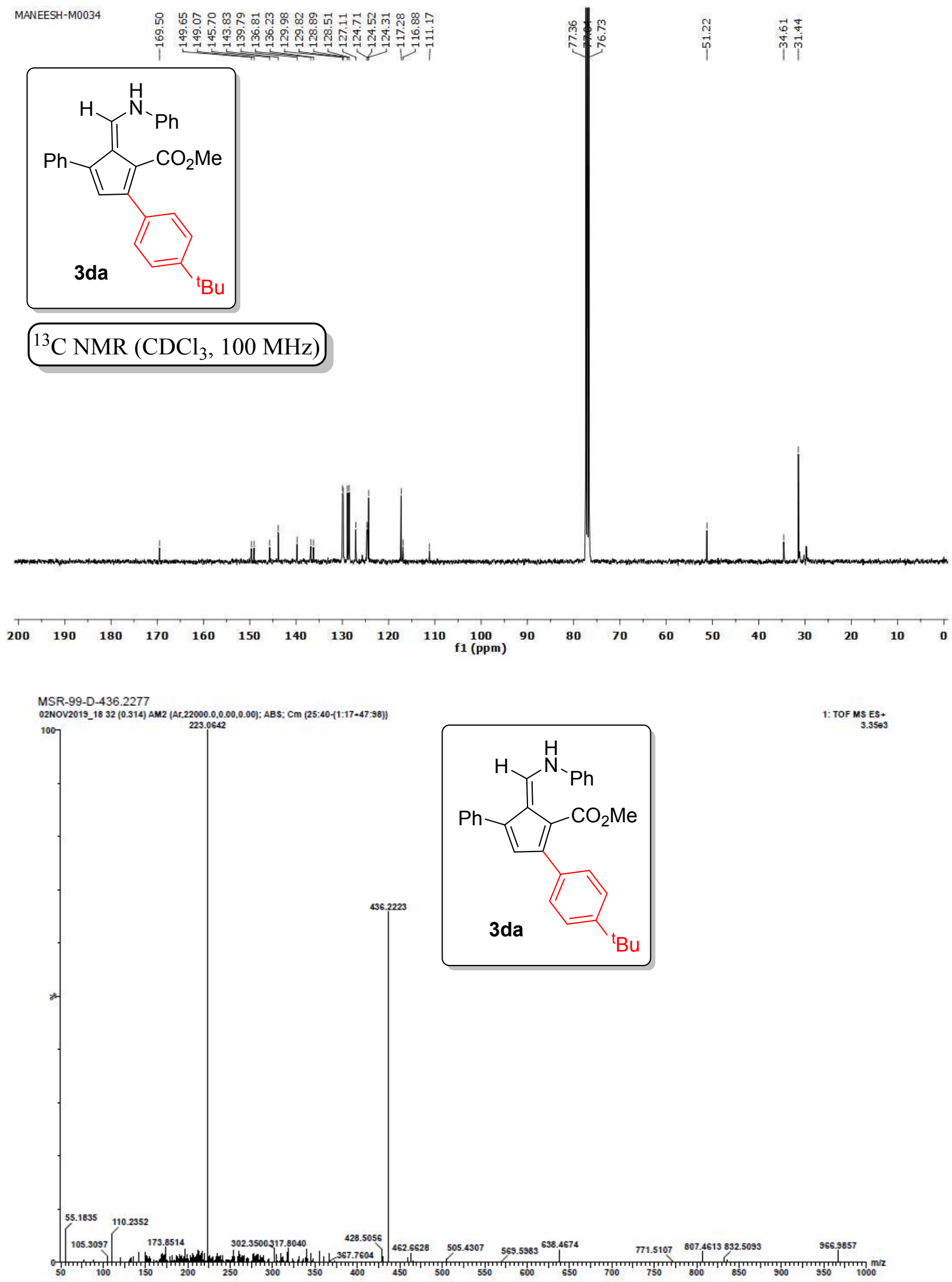

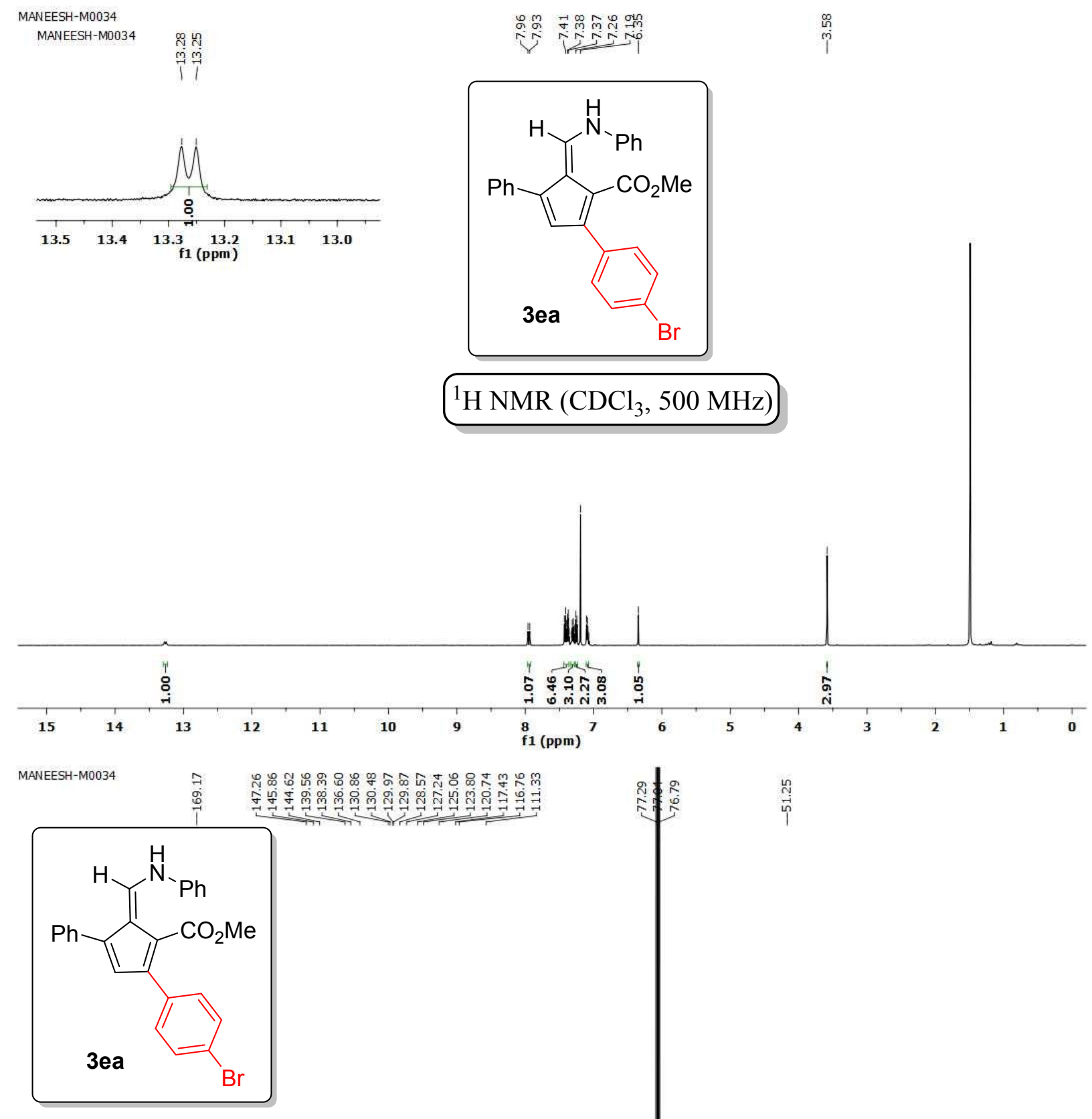

${ }^{13} \mathrm{C} \mathrm{NMR}\left(\mathrm{CDCl}_{3}, 125 \mathrm{MHz}\right)$

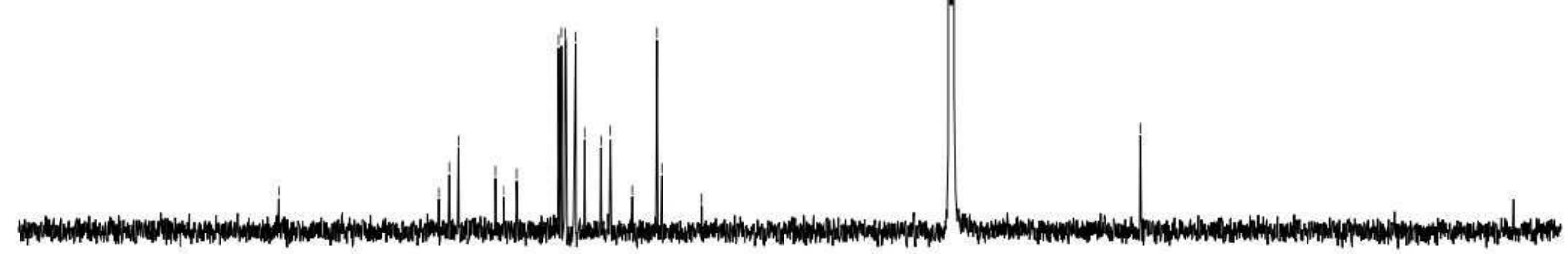

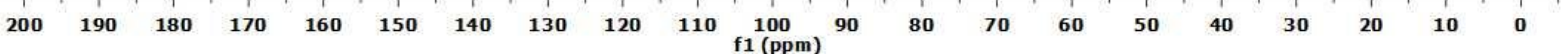



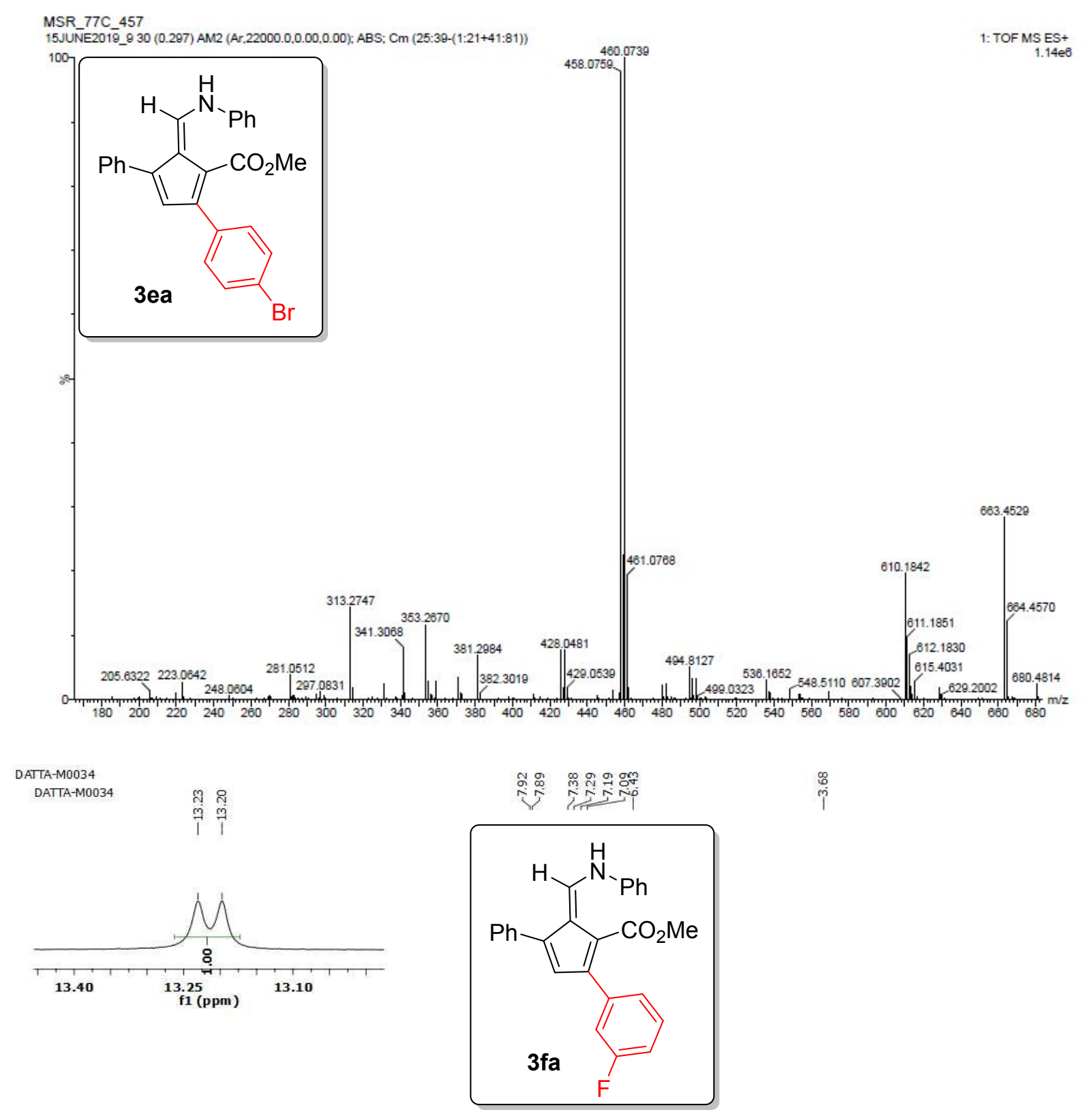

${ }^{1} \mathrm{H} \mathrm{NMR}\left(\mathrm{CDCl}_{3}, 400 \mathrm{MHz}\right)$

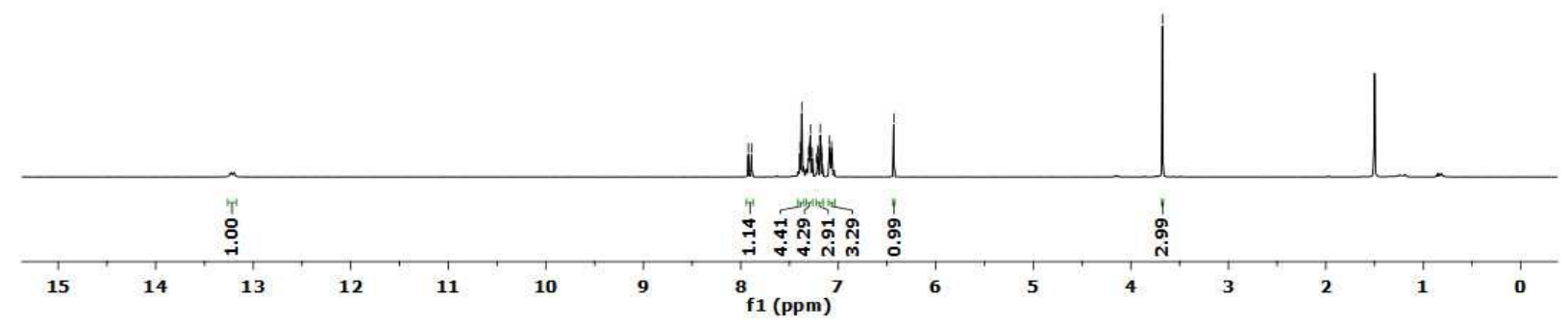



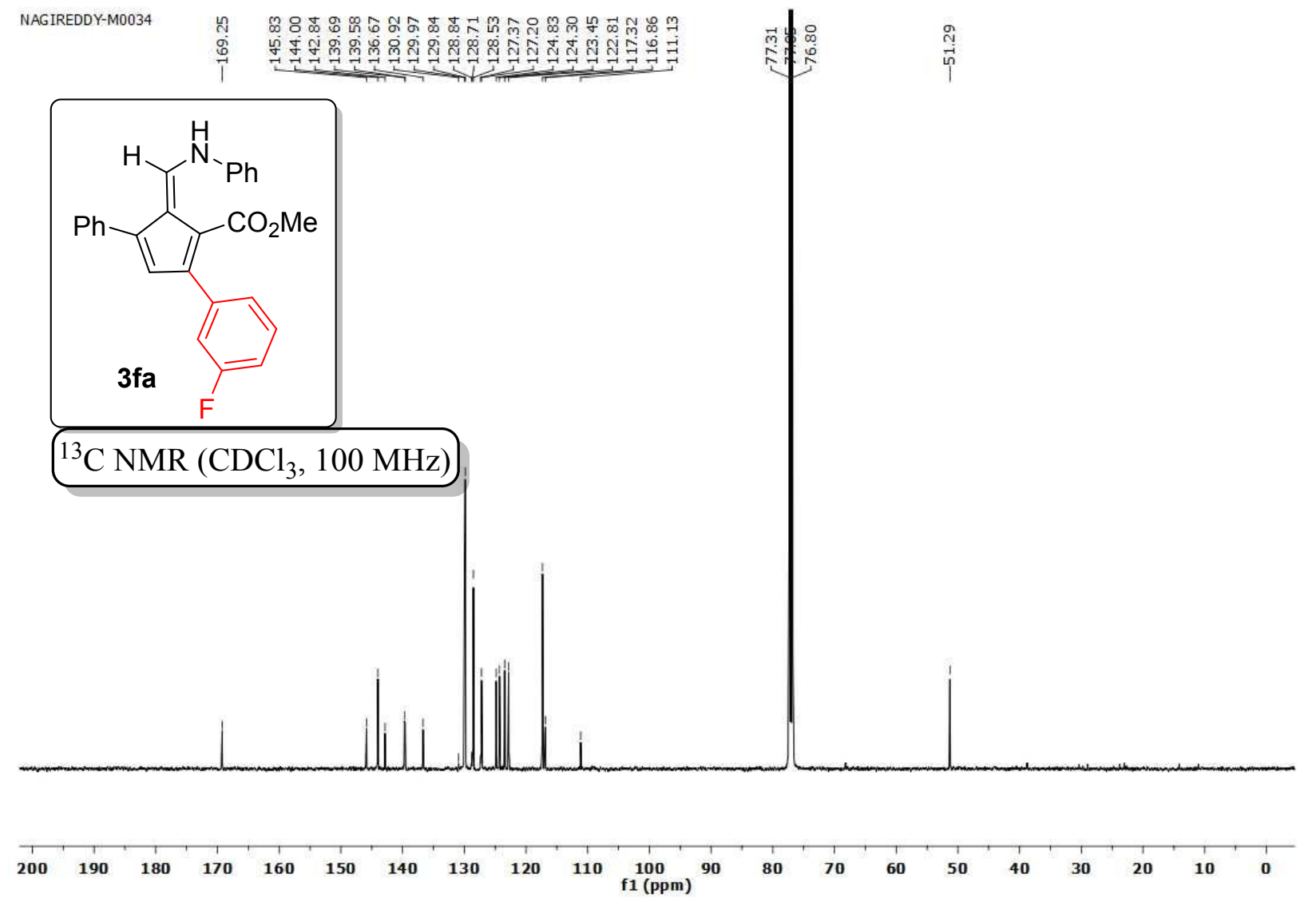

NAG IREDDY-M0034

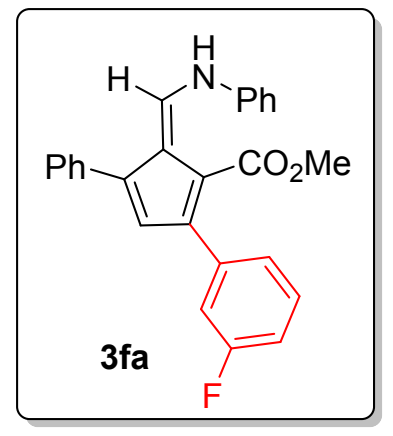

${ }^{19} \mathrm{~F} \mathrm{NMR}\left(\mathrm{CDCl}_{3}, 377 \mathrm{MHz}\right)$

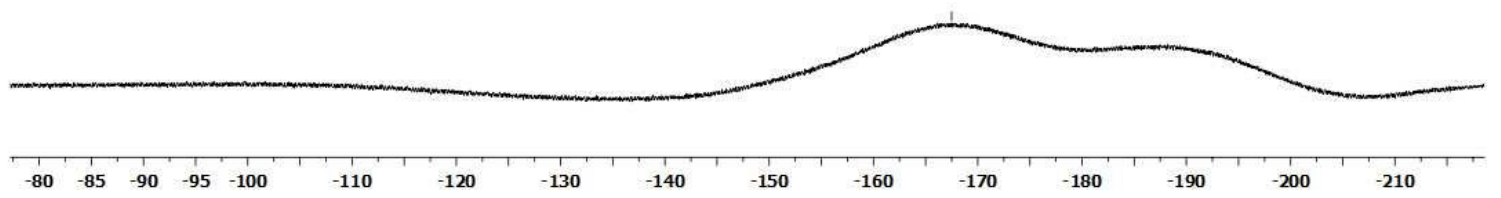


MSR_75C_397

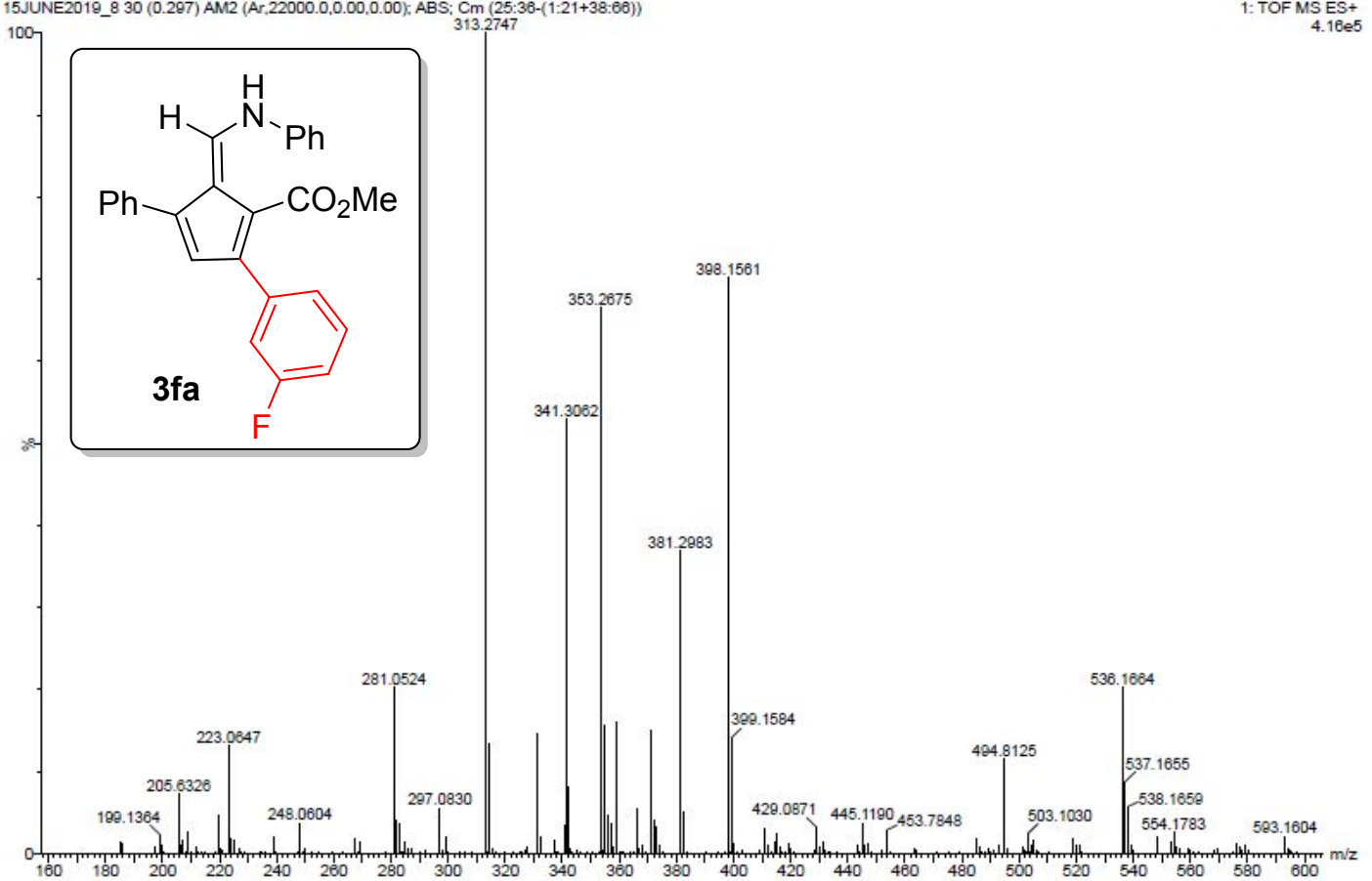




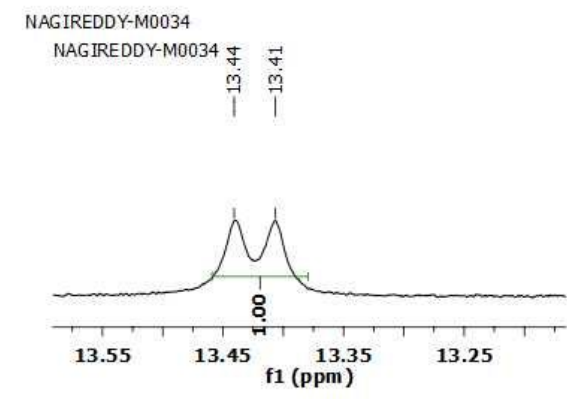

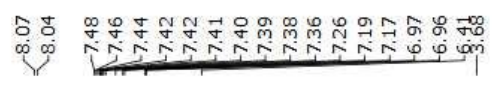

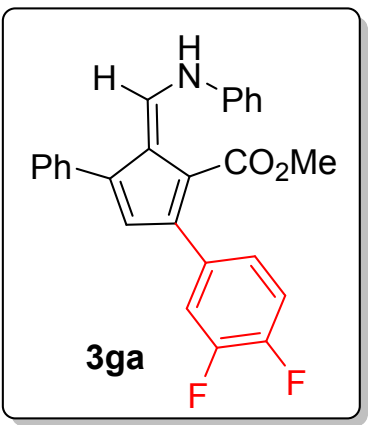

${ }^{1} \mathrm{H} \mathrm{NMR}\left(\mathrm{CDCl}_{3}, 400 \mathrm{MHz}\right)$
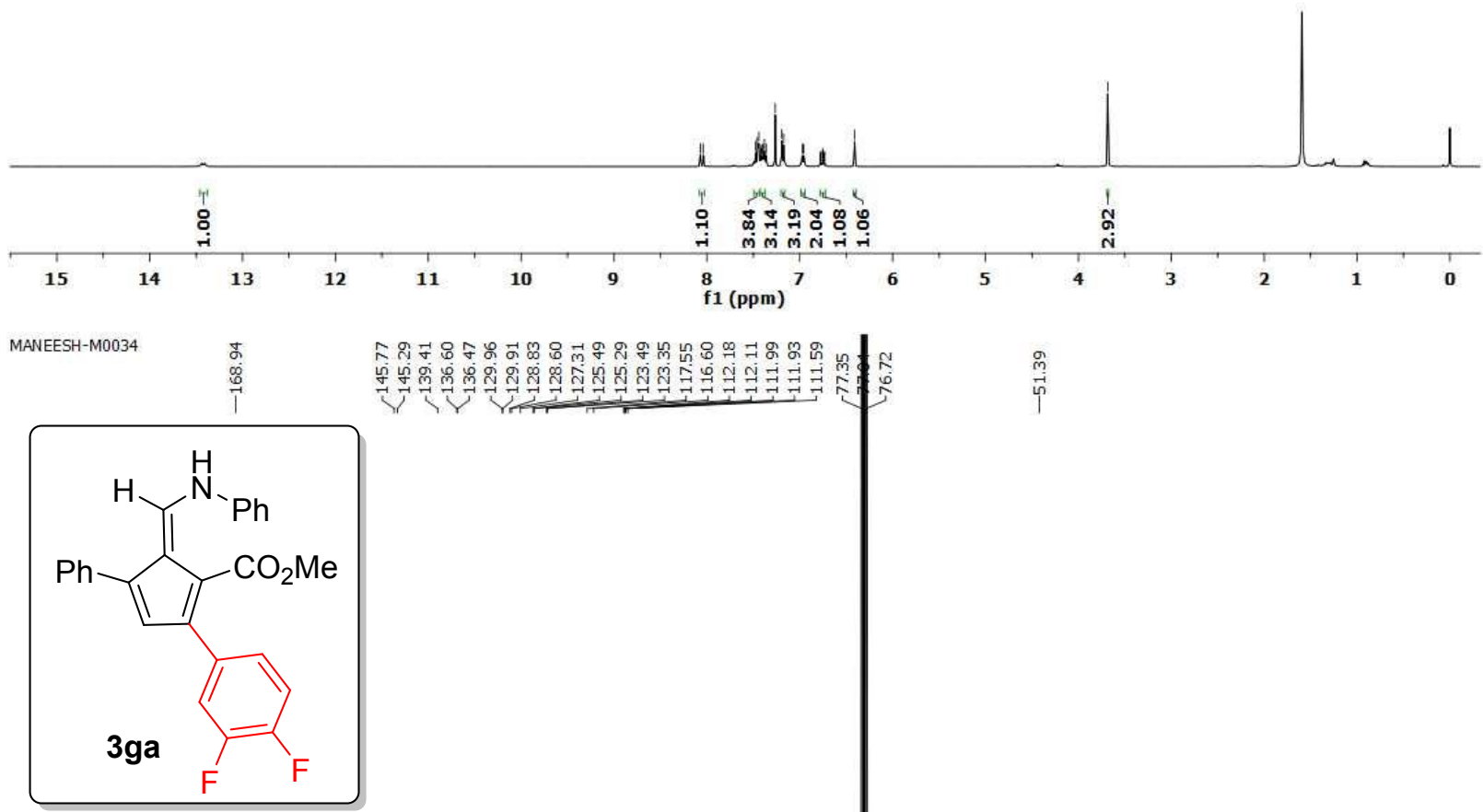

$\stackrel{\substack{m \\-1}}{\text { फल }}$

${ }^{13} \mathrm{C} \mathrm{NMR}\left(\mathrm{CDCl}_{3}, 100 \mathrm{MHz}\right)$
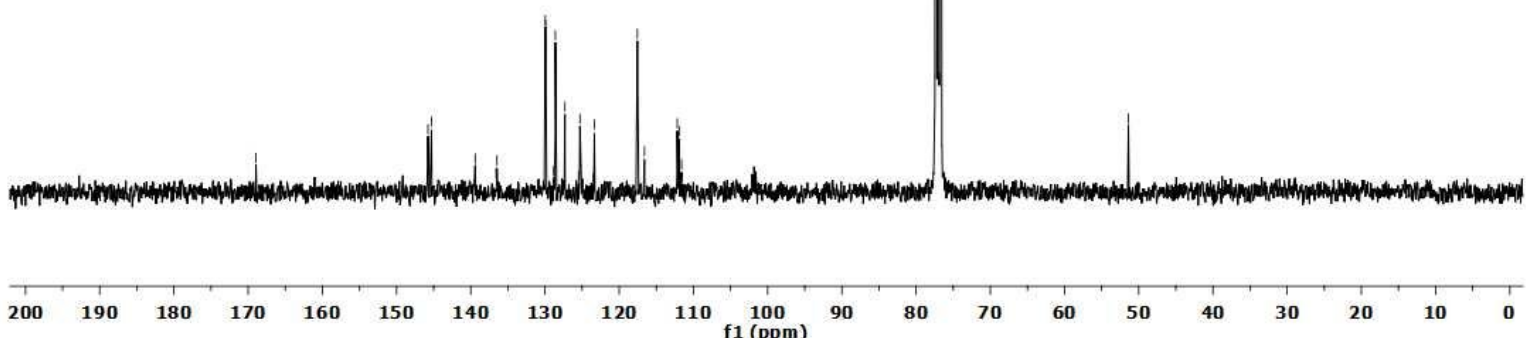


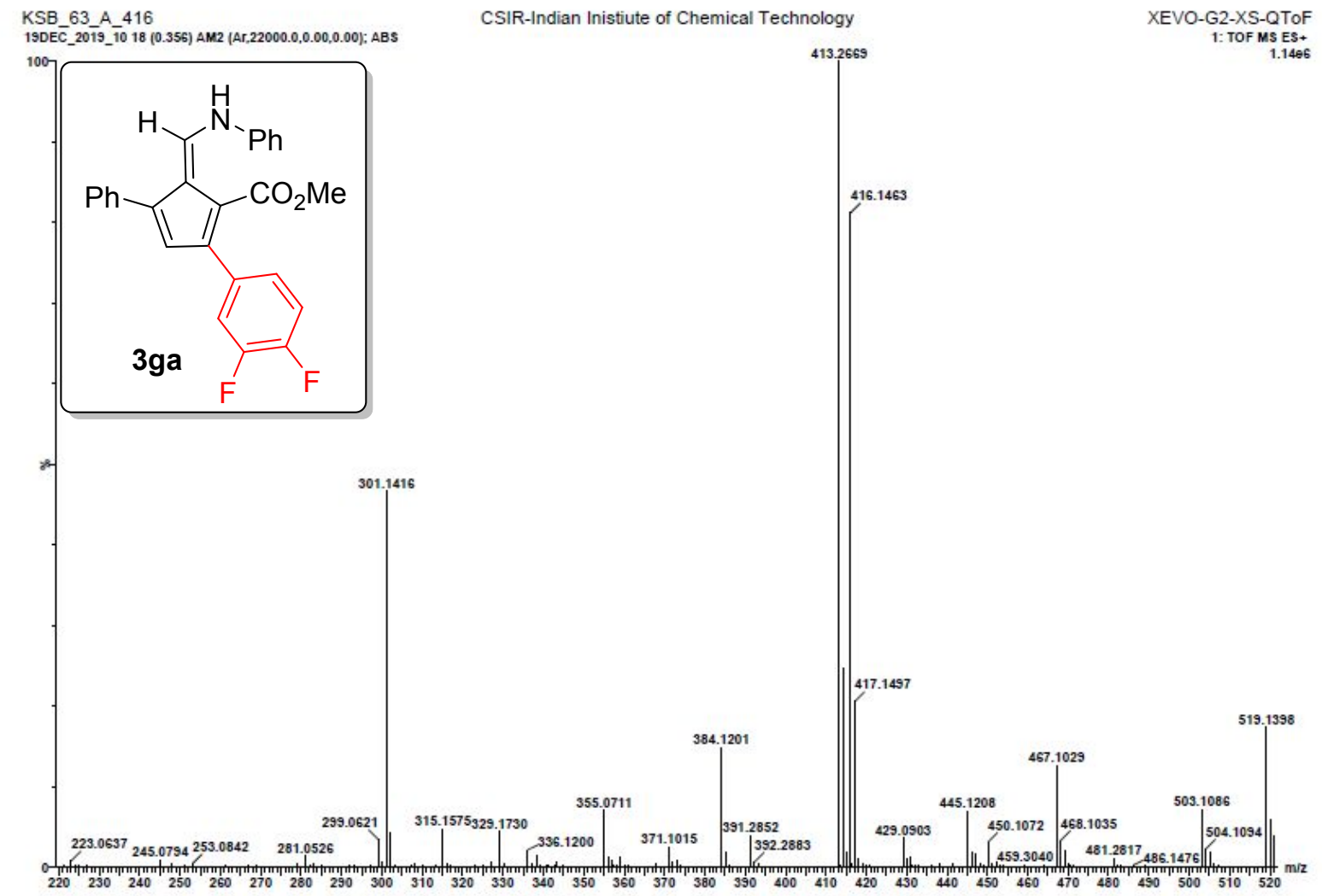

MANEESH-H0011

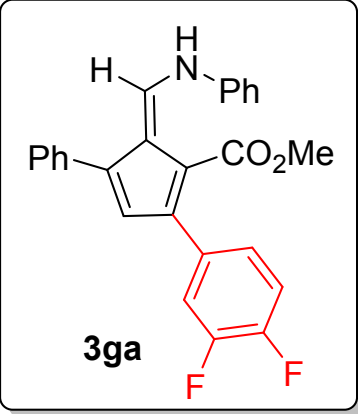

${ }^{19} \mathrm{~F} \mathrm{NMR}\left(\mathrm{CDCl}_{3}, 377 \mathrm{MHz}\right)$

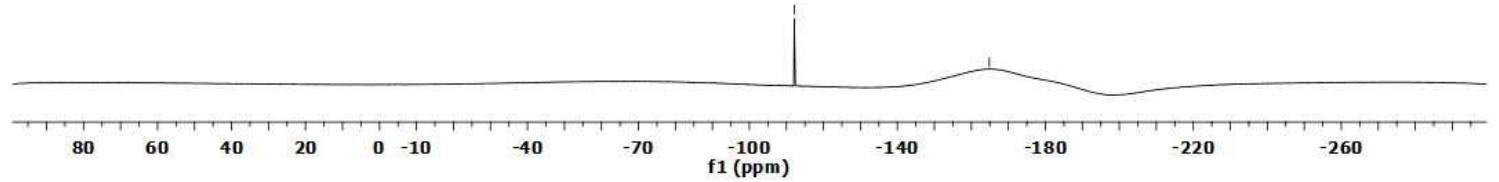




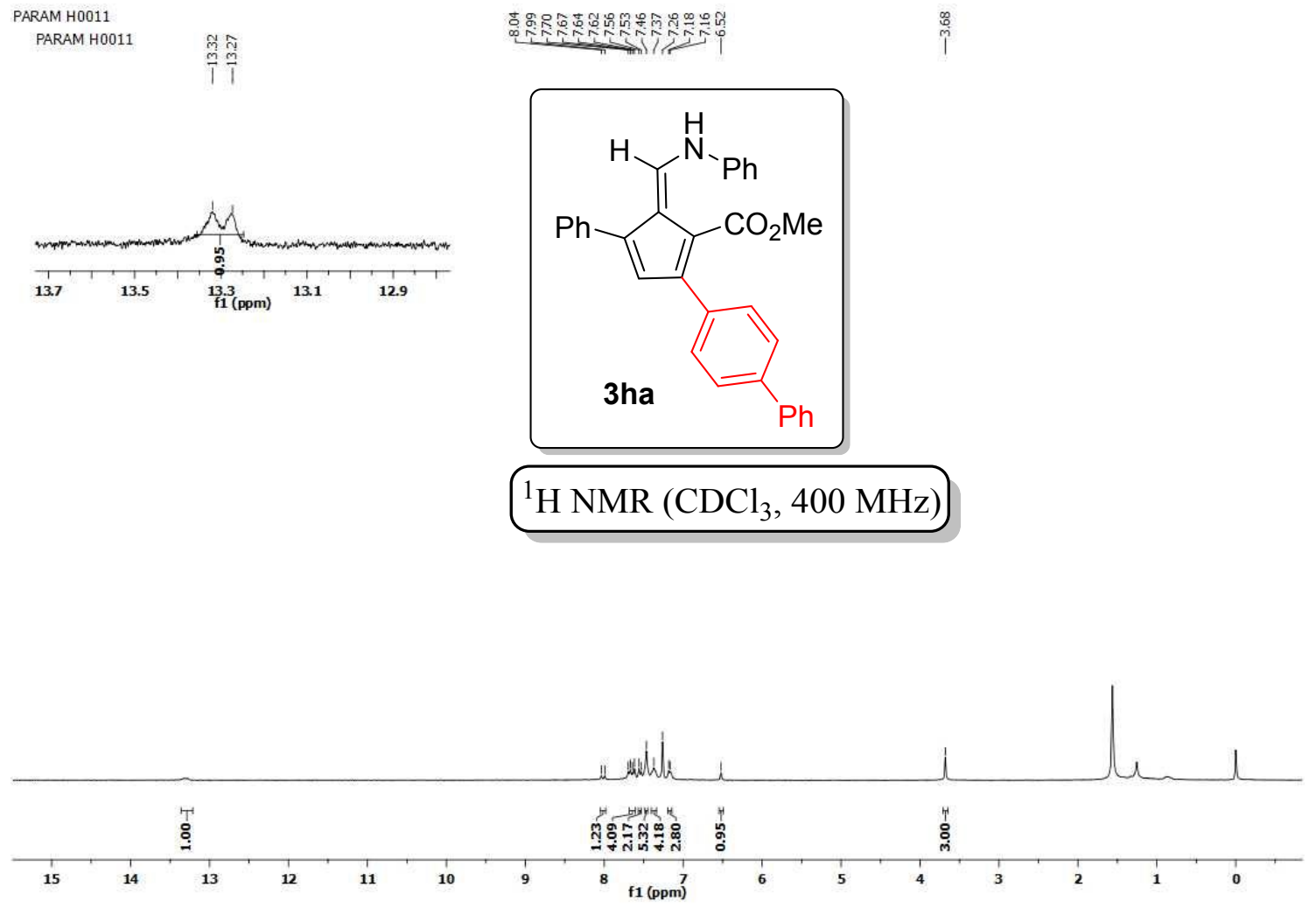



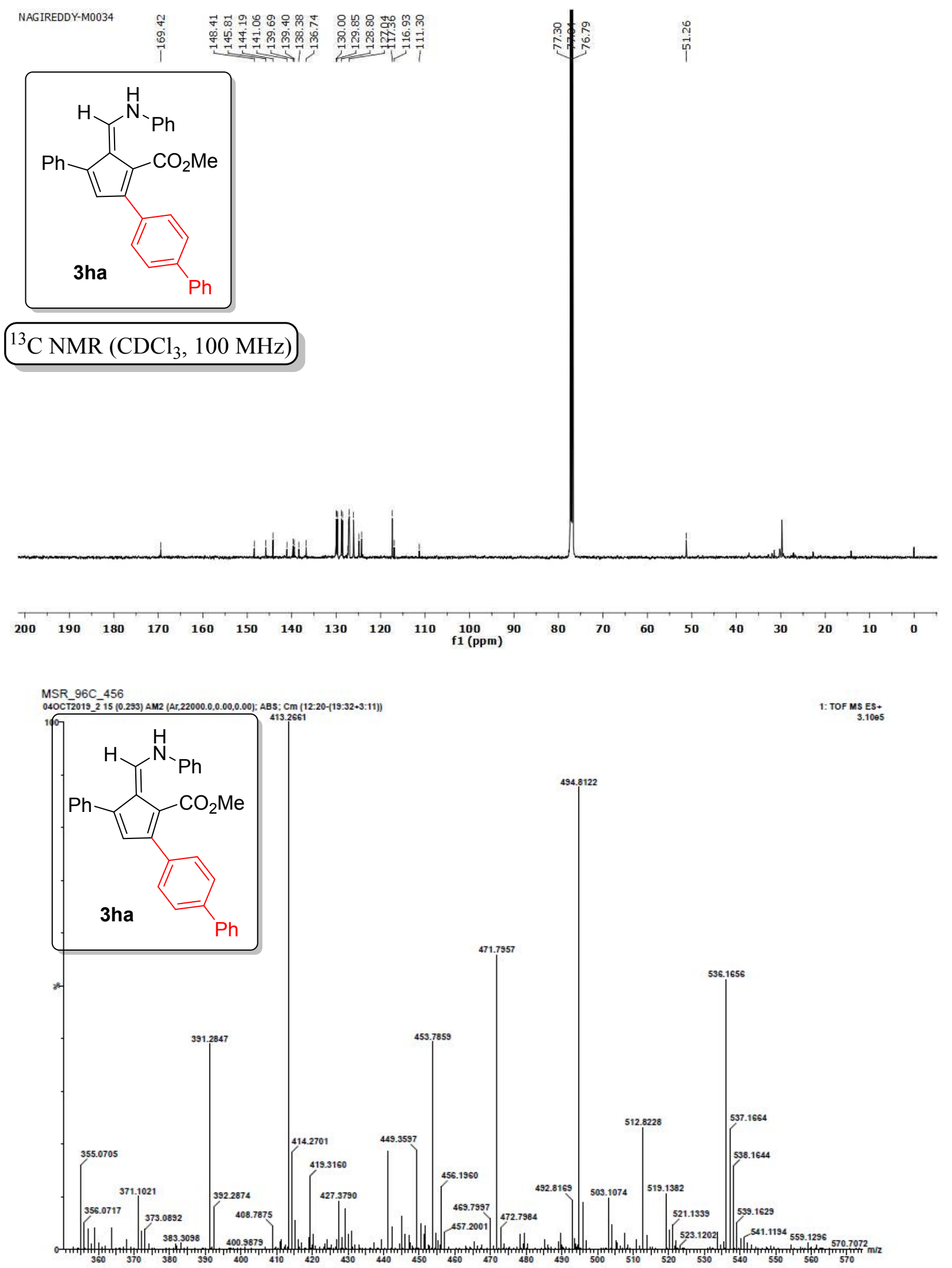

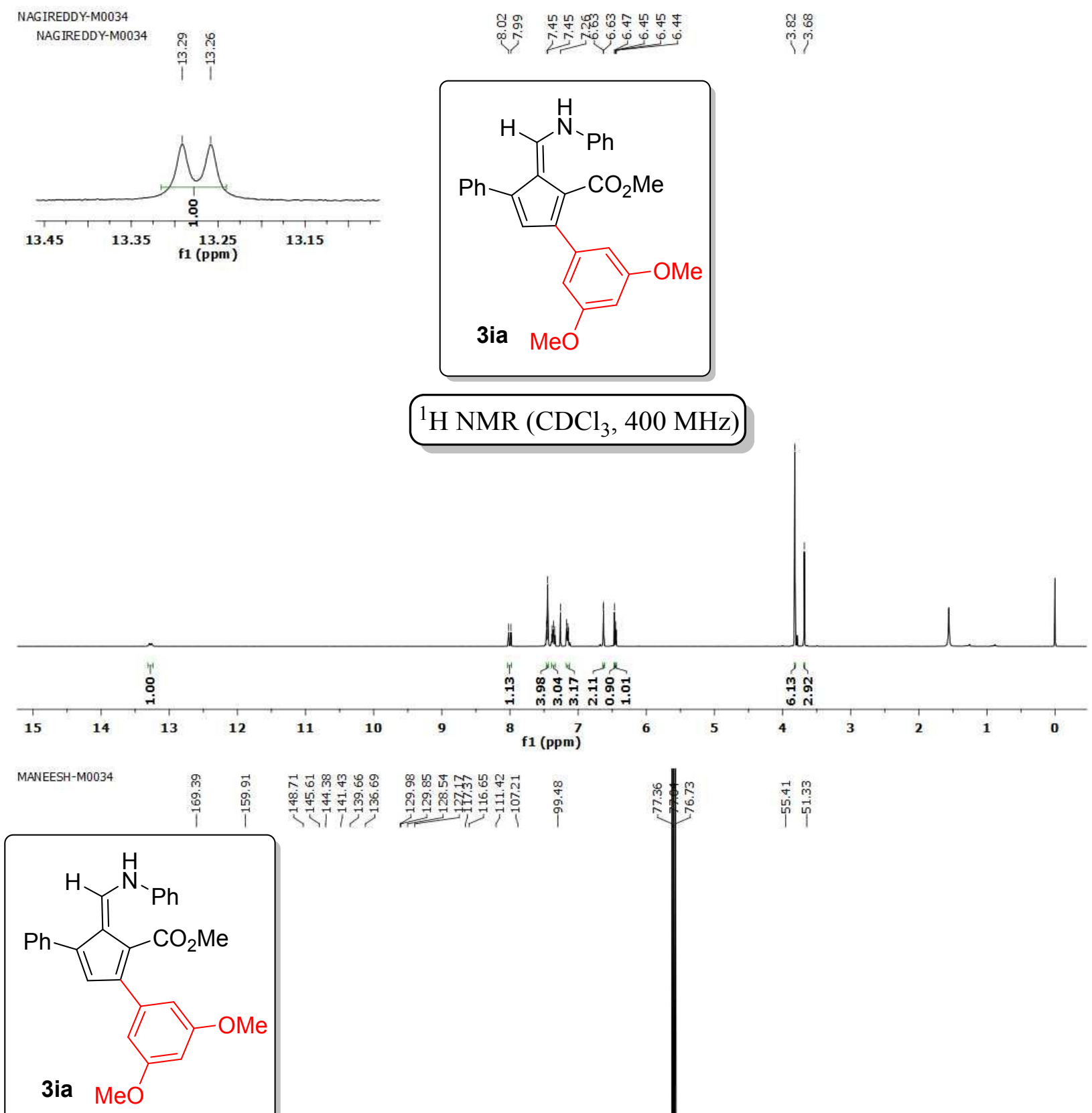

${ }^{13} \mathrm{C} \mathrm{NMR}\left(\mathrm{CDCl}_{3}, 100 \mathrm{MHz}\right)$

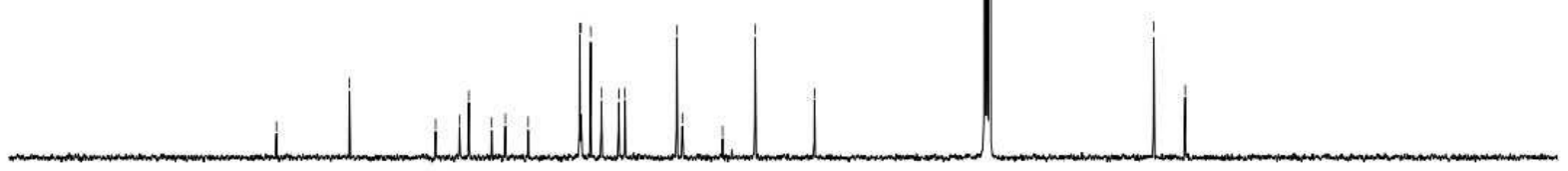

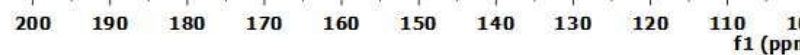


Maddi SRIDHAR REDDY

11/27/2019 4:29:09 PM

ThermoScientific EXACTIVE ORBITRAP

Analysed By G Saikrishna

MSR-101-C \#2-13 ${ }^{-} \mathrm{RT}^{-}{ }^{-} 0.01-0.05^{-} \mathrm{AV}^{-}{ }^{-}{ }^{-}{ }^{-} \mathrm{NL}:{ }^{-} 1.18 \mathrm{E} 8$

T: FTMS $\{1,1\}+p$ APCl corona Full ms [100.00-2000.00]

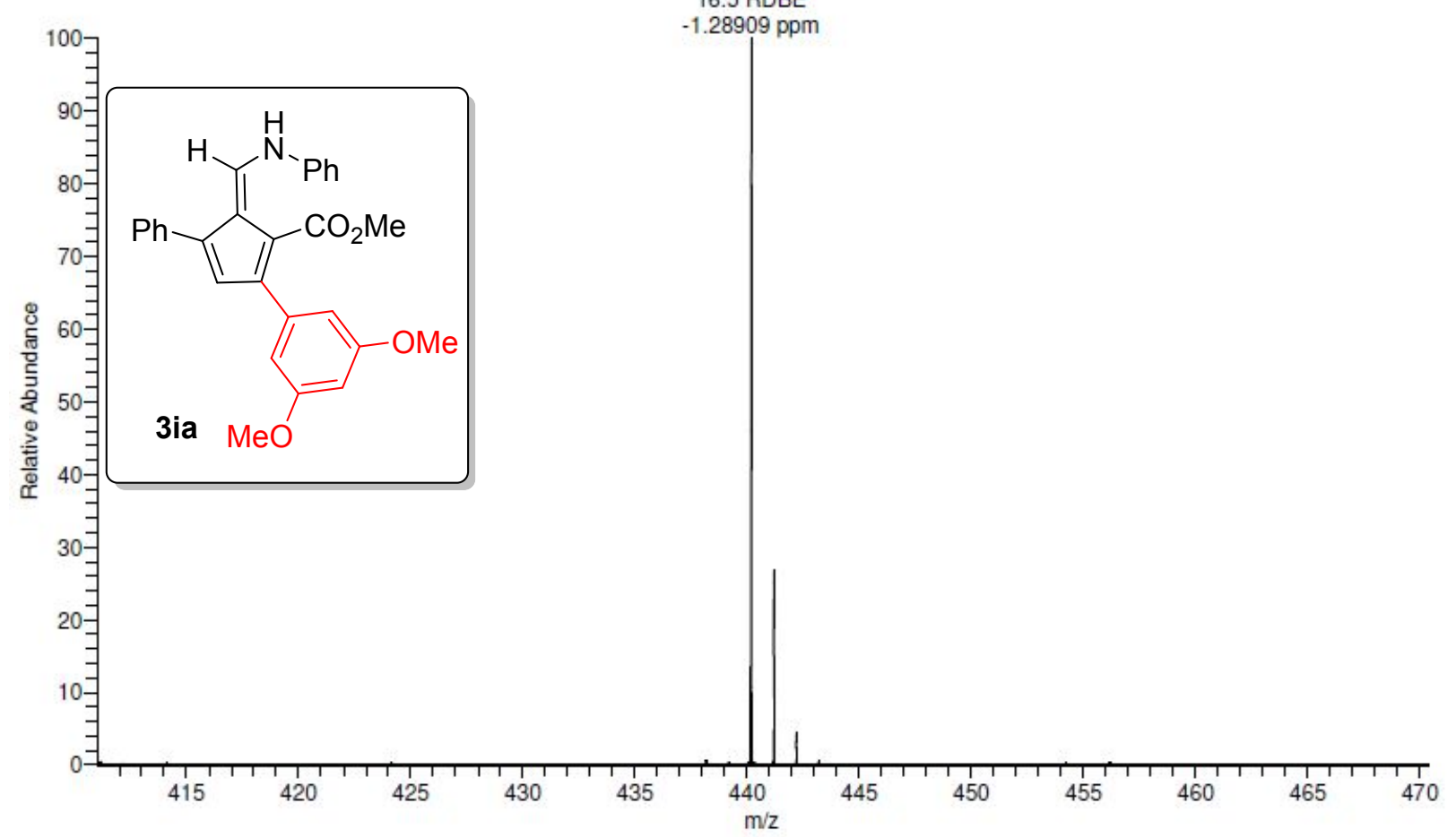

NAGIREDDY-M0034

NAGIREDDY-M0034 青

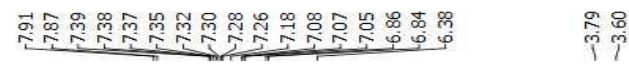
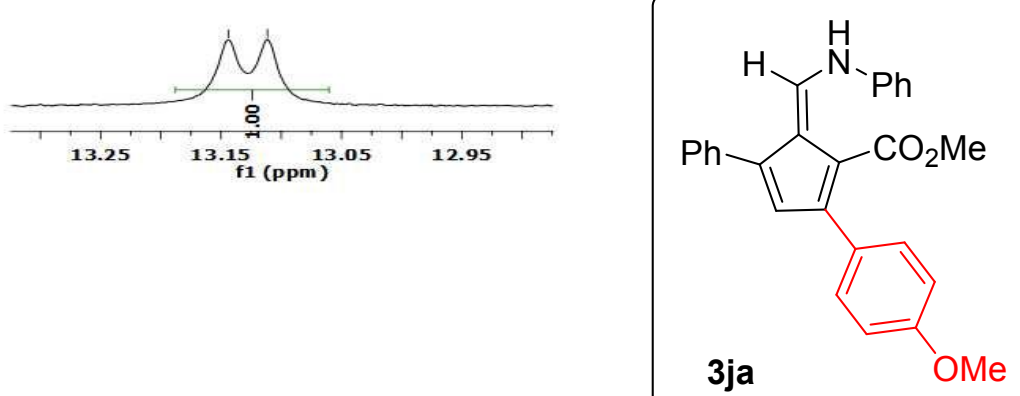

${ }^{1} \mathrm{H} \mathrm{NMR}\left(\mathrm{CDCl}_{3}, 500 \mathrm{MHz}\right)$

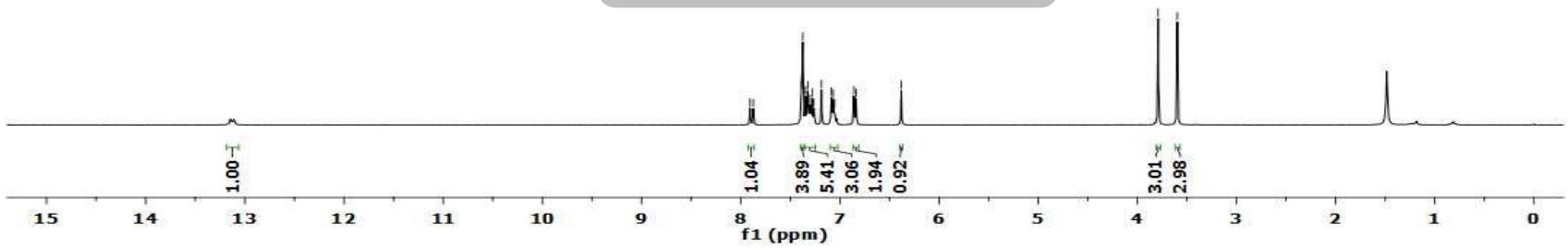



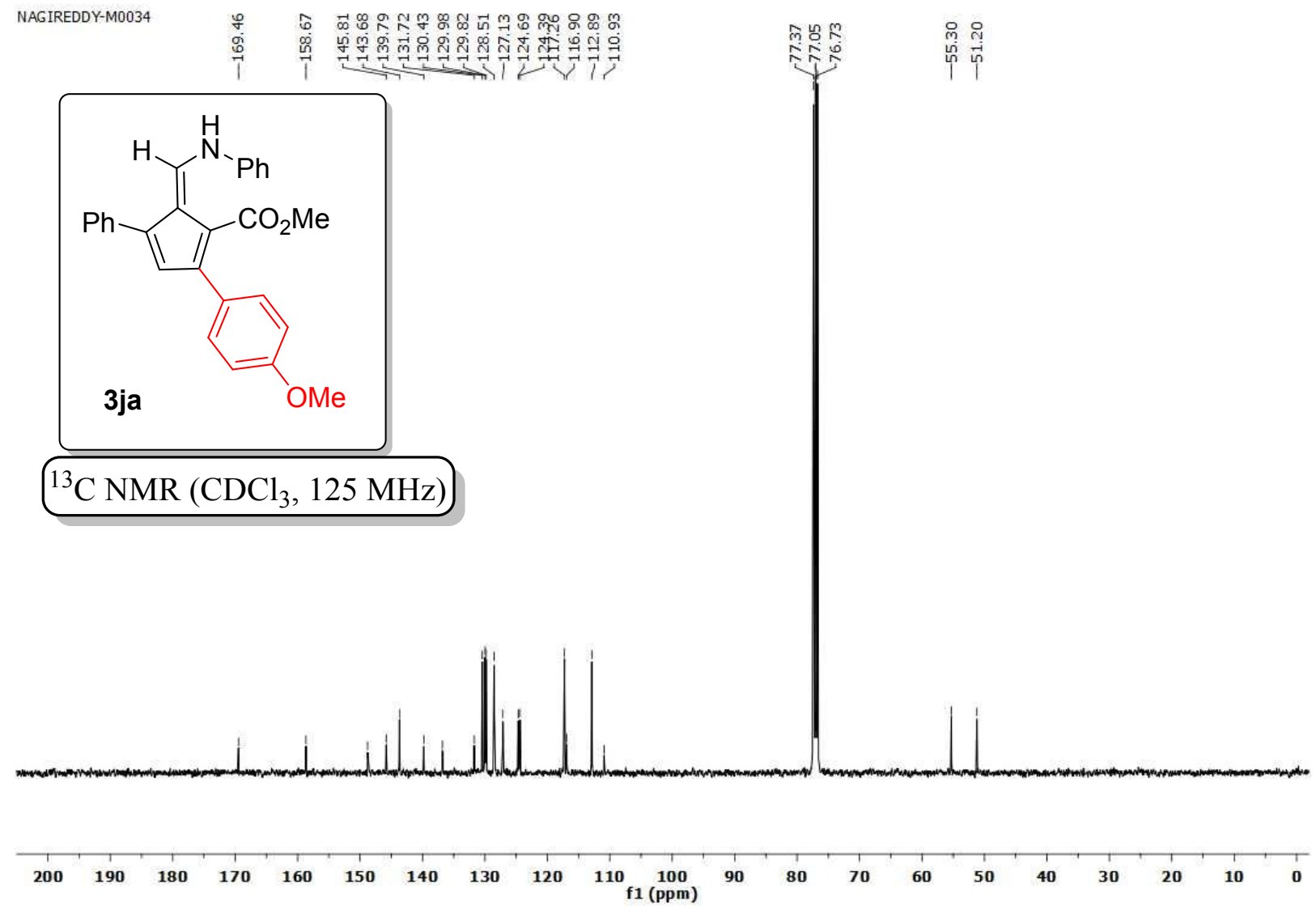

Maddi SRIDHAR REDDY

ThermoScientific EXACTIVE ORBITRAP
Analysed By G Saikrishna

MSR-80-D \#2-13 RT: 0.02-0.05 AV: 12 NL: 7.05E8

T: FTMS $\{1,1\}+p$ APCI corona Full ms [100.00-2000.00]

$\mathrm{C}_{27} \mathrm{H}_{24} \mathrm{O}_{3} \mathrm{~N}=410.17507$

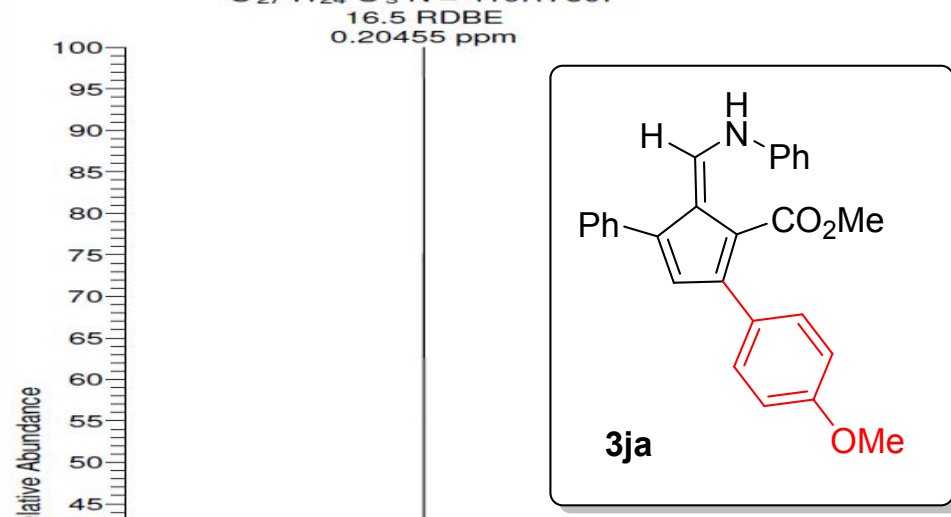

$\frac{\text { क्ष }}{\text { क्ष }}$

40

35

30

25当

20 当

15

10 当

扫,

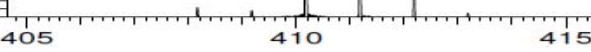

415

$\mathrm{m} / \mathrm{z}$ 

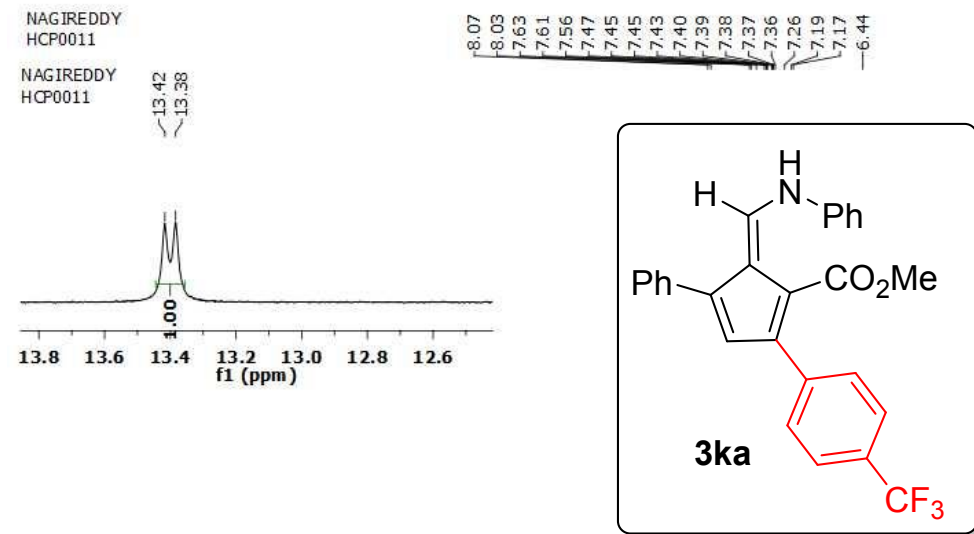

${ }^{1} \mathrm{H} \mathrm{NMR}\left(\mathrm{CDCl}_{3}, 400 \mathrm{MHz}\right)$

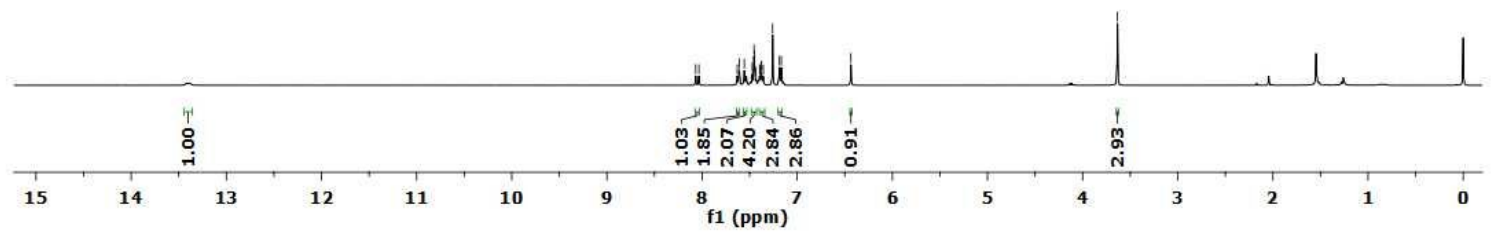

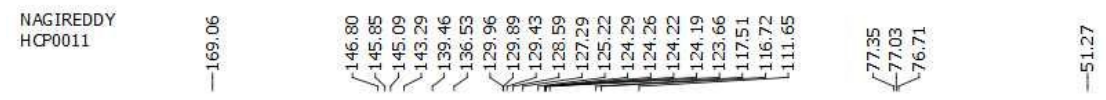

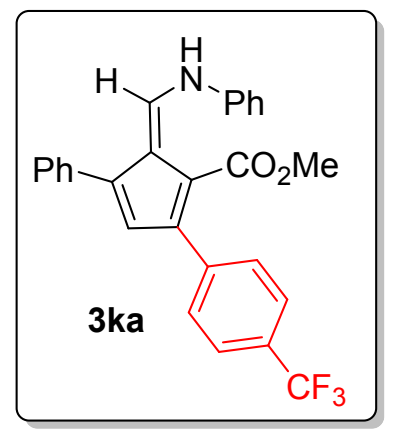

${ }^{13} \mathrm{C} \mathrm{NMR}\left(\mathrm{CDCl}_{3}, 100 \mathrm{MHz}\right)$
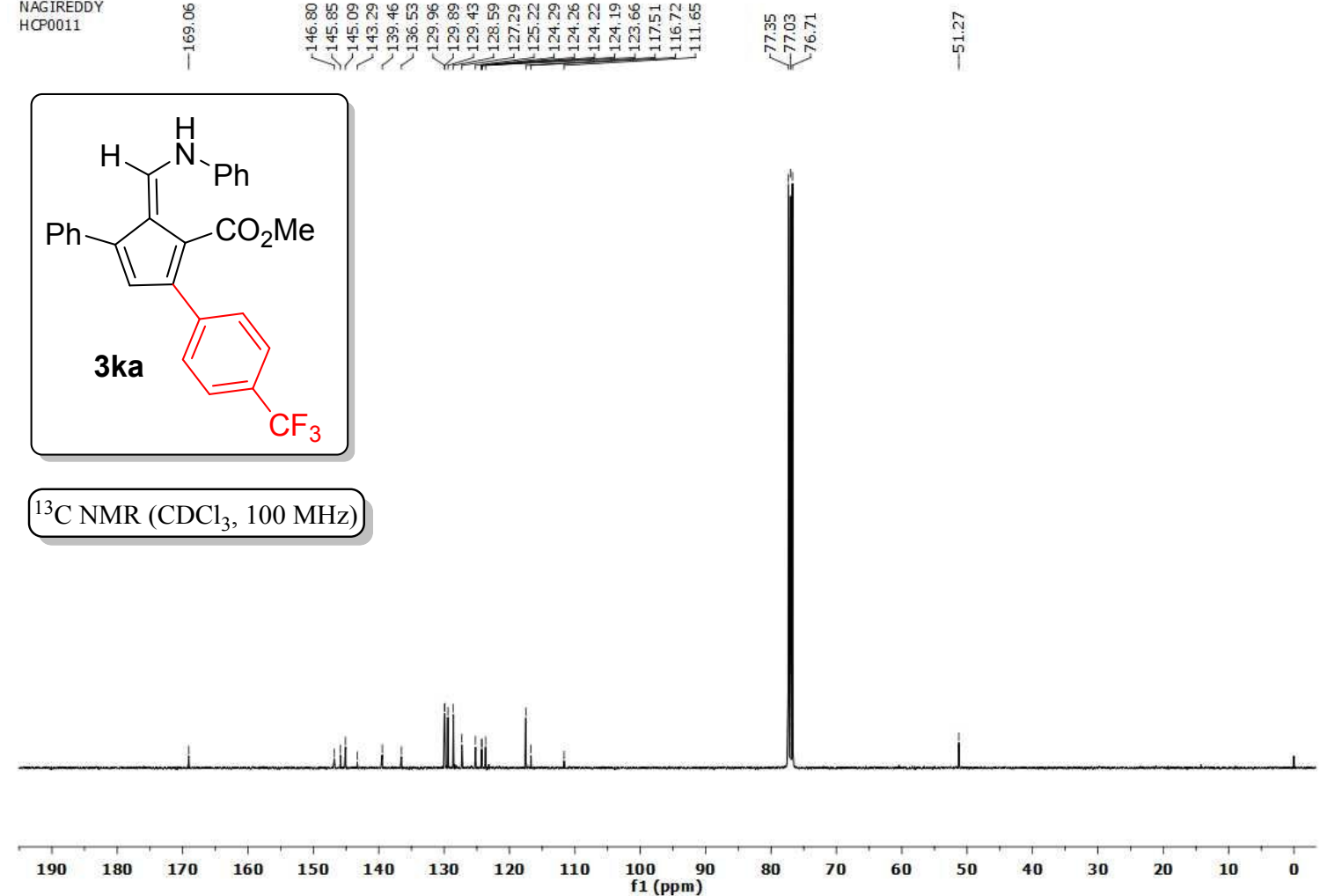


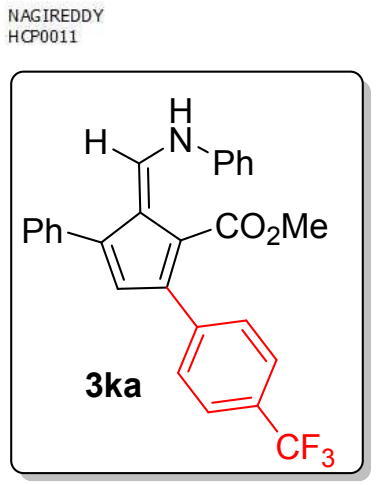

${ }^{19} \mathrm{~F} \mathrm{NMR}\left(\mathrm{CDCl}_{3}, 377 \mathrm{MHz}\right)$

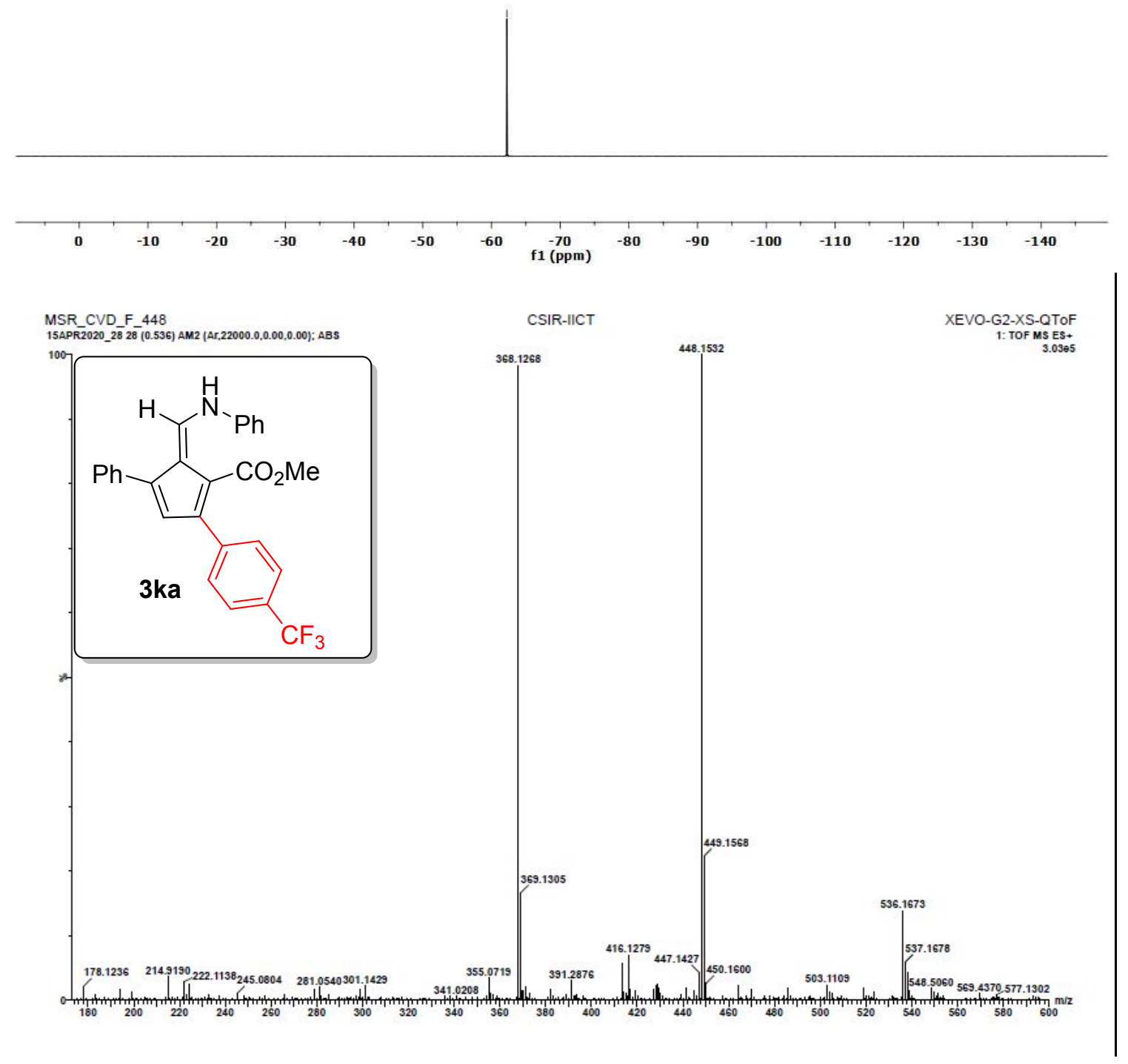




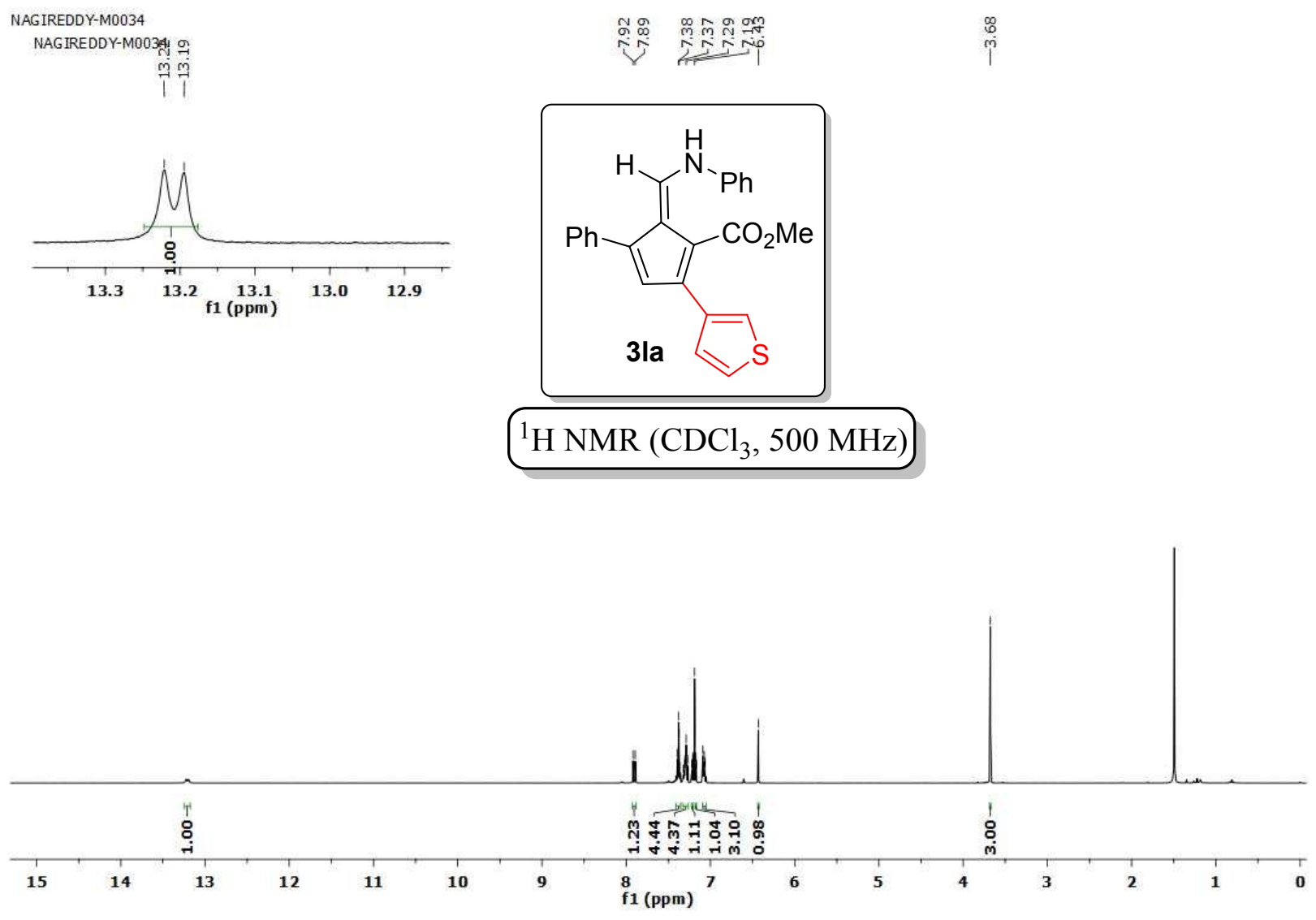

MANEESH-HOO11

ำ

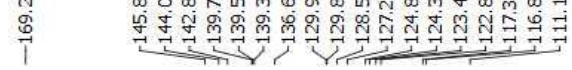<smiles>COC(=O)C1=C(c2ccsc2)C=C(P)/C1=C/NPc1ccccc1</smiles>

${ }^{13} \mathrm{C} \mathrm{NMR}\left(\mathrm{CDCl}_{3}, 125 \mathrm{MHz}\right)$
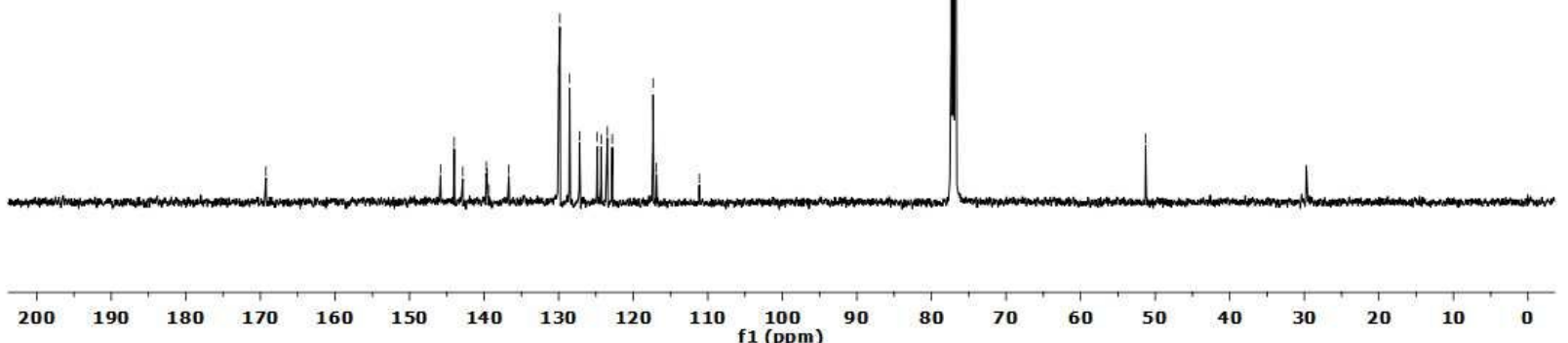


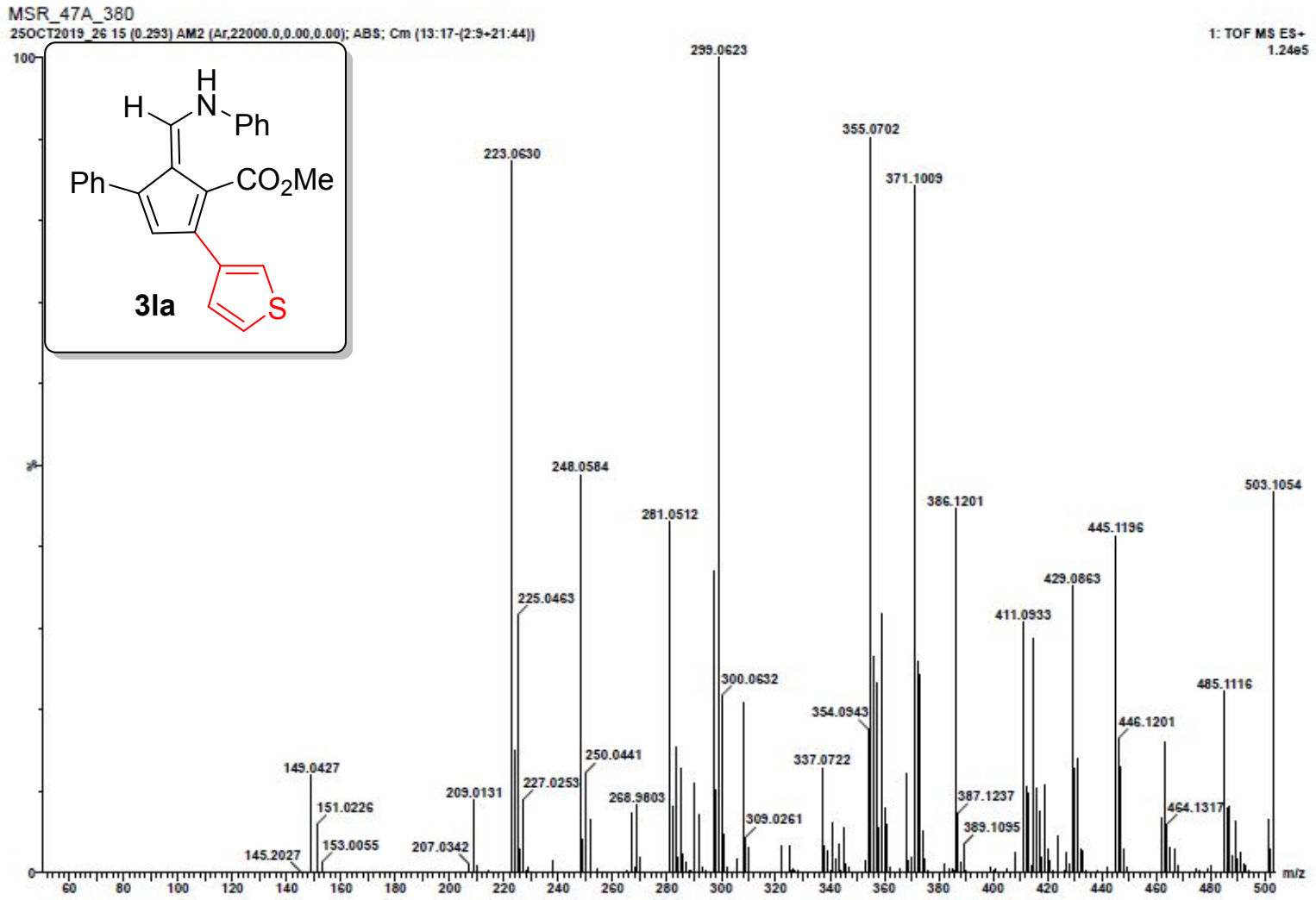




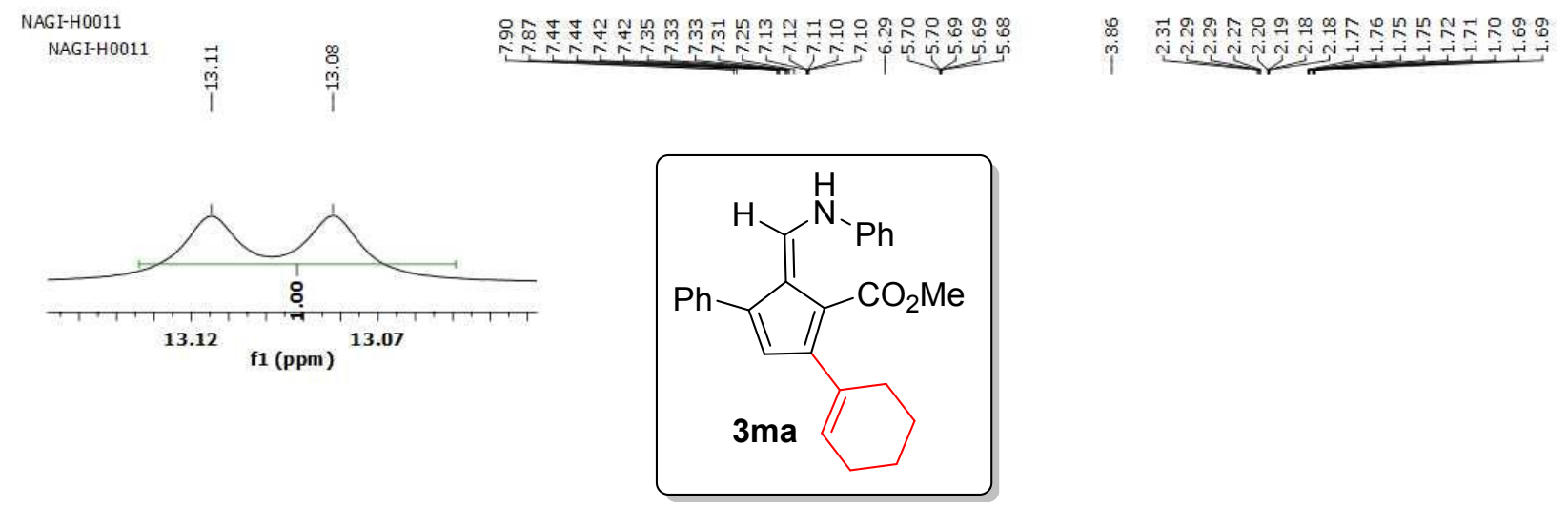

${ }^{1} \mathrm{H} \mathrm{NMR}\left(\mathrm{CDCl}_{3}, 400 \mathrm{MHz}\right)$

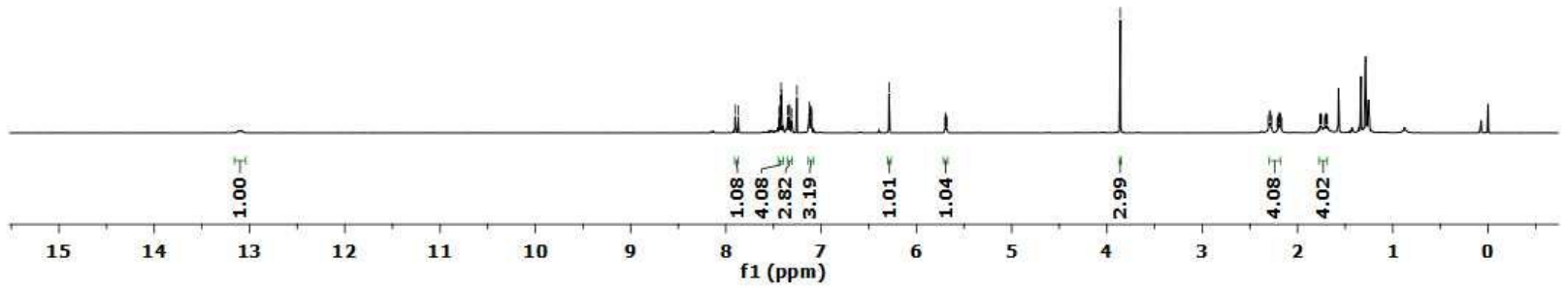

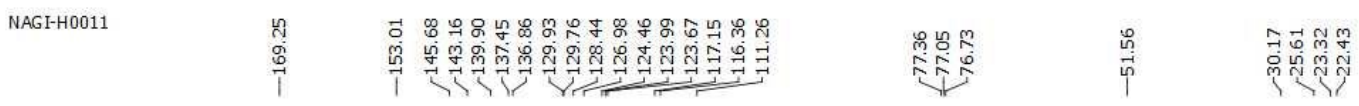<smiles>COC(=O)C1=C(C2=CCCCC2)C=C(Pc2ccccc2)/C1=C/Nc1ccccc1</smiles>

${ }^{13} \mathrm{C} \mathrm{NMR}\left(\mathrm{CDCl}_{3}, 100 \mathrm{MHz}\right)$
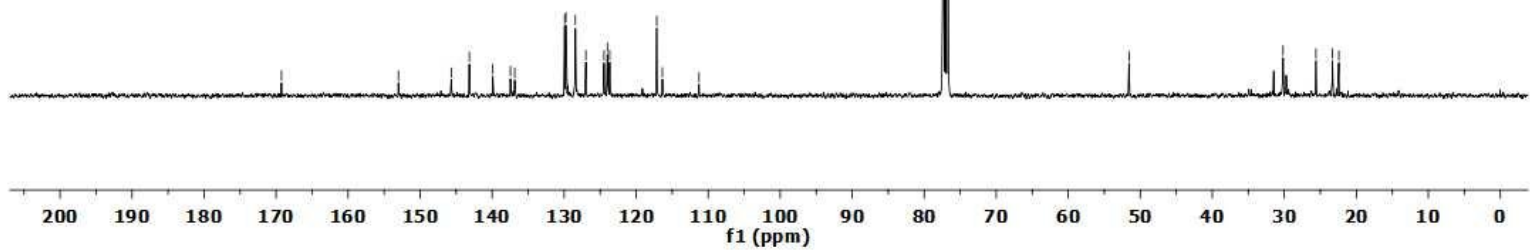

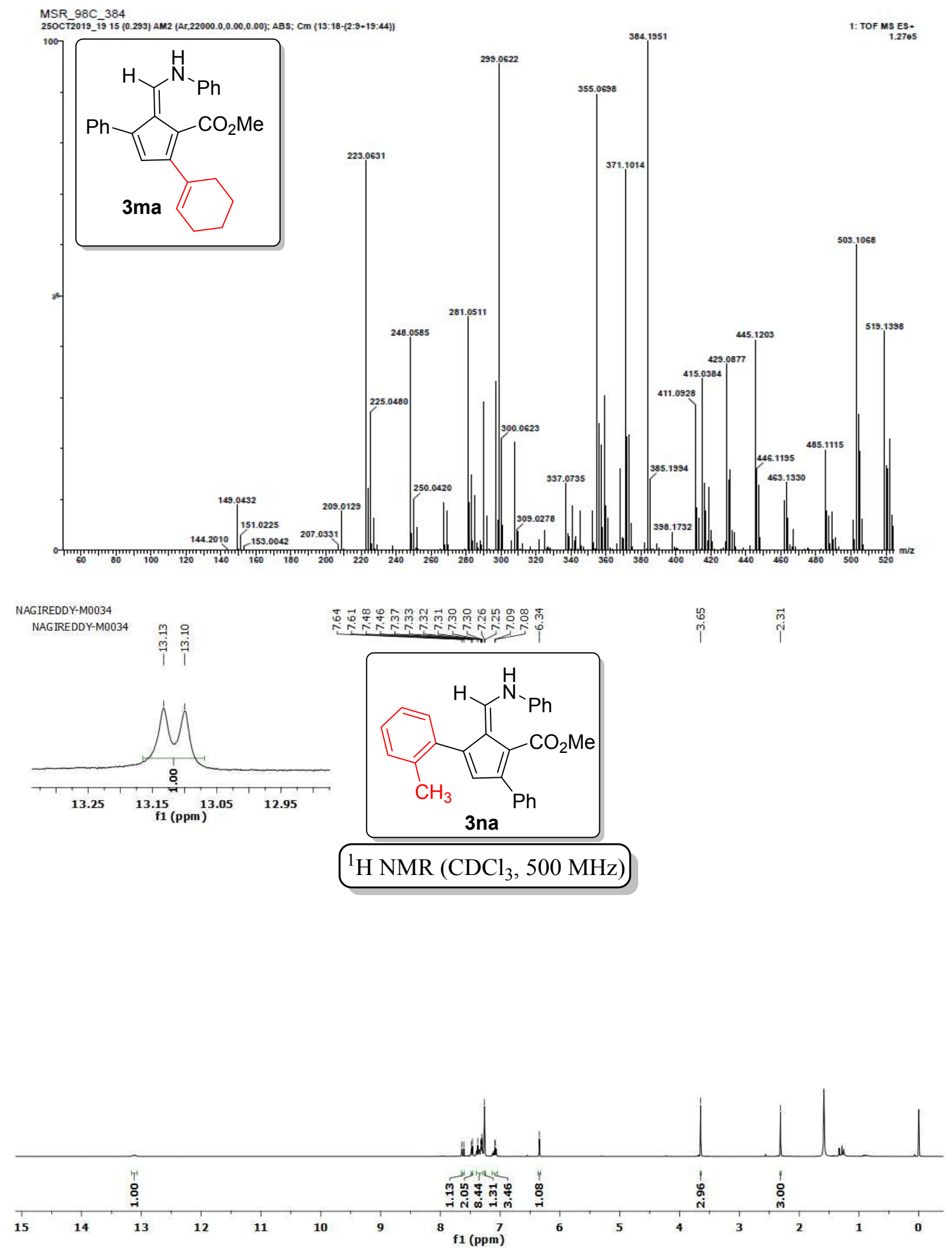


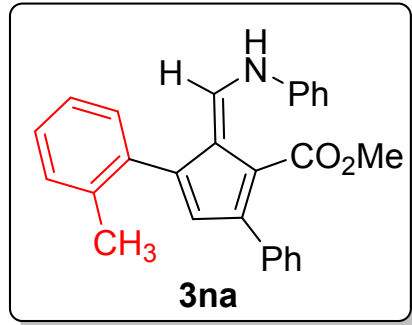

${ }^{13} \mathrm{C} \mathrm{NMR}\left(\mathrm{CDCl}_{3}, 125 \mathrm{MHz}\right)$
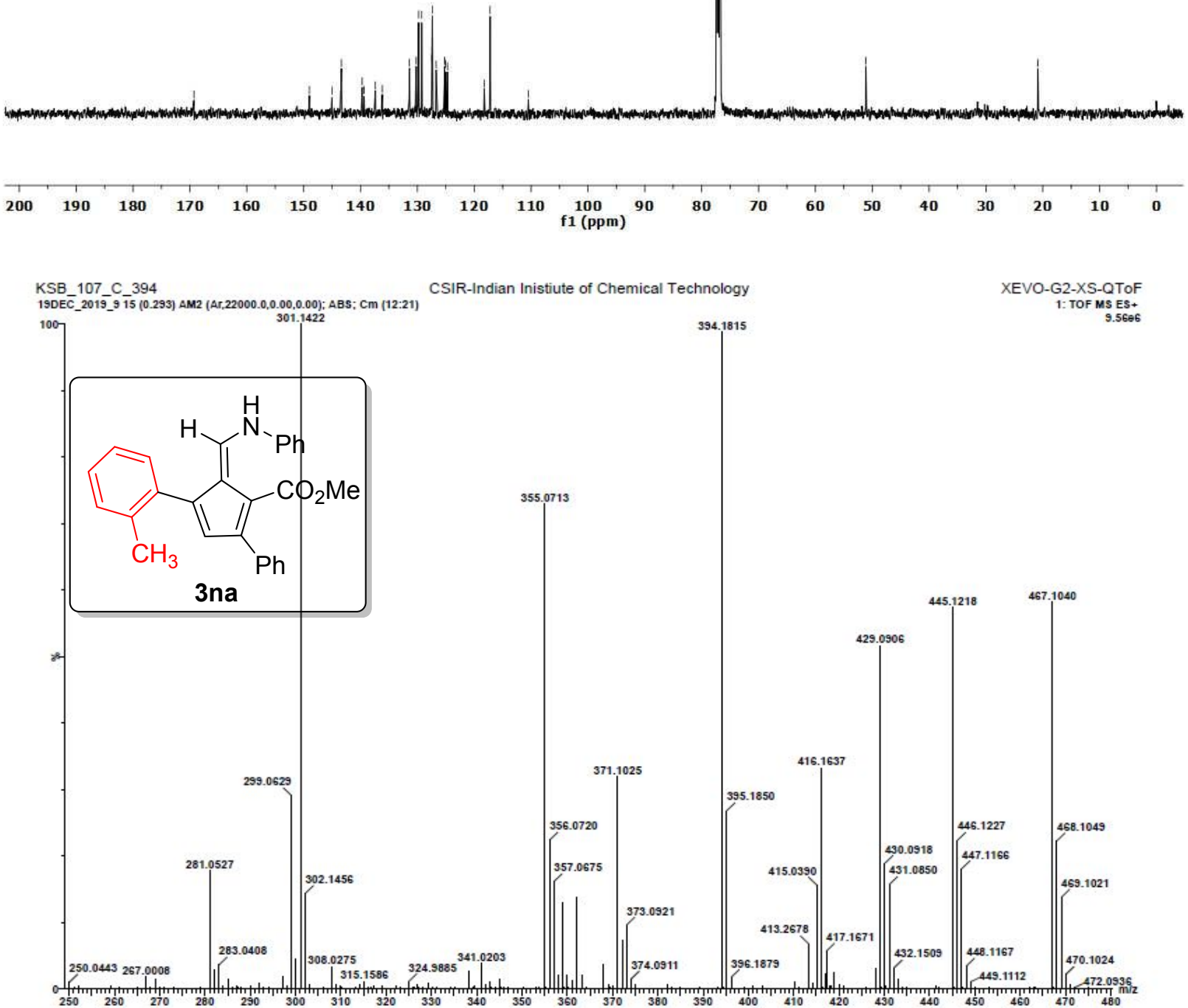


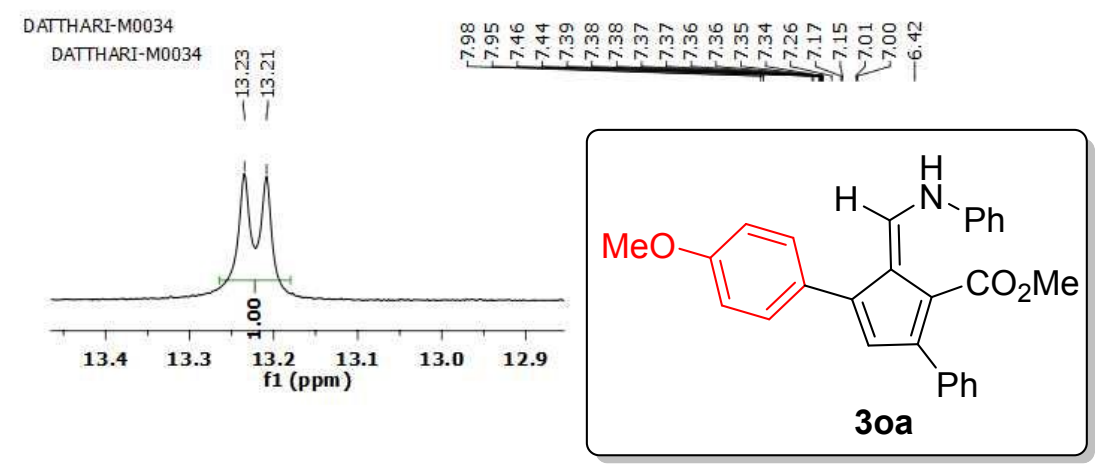

$\begin{array}{ll}\infty & 0 \\ 0 & 0 \\ 0 & 0 \\ 1 & 1\end{array}$

${ }^{1} \mathrm{H}$ NMR $\left(\mathrm{CDCl}_{3}, 400 \mathrm{MHz}\right)$
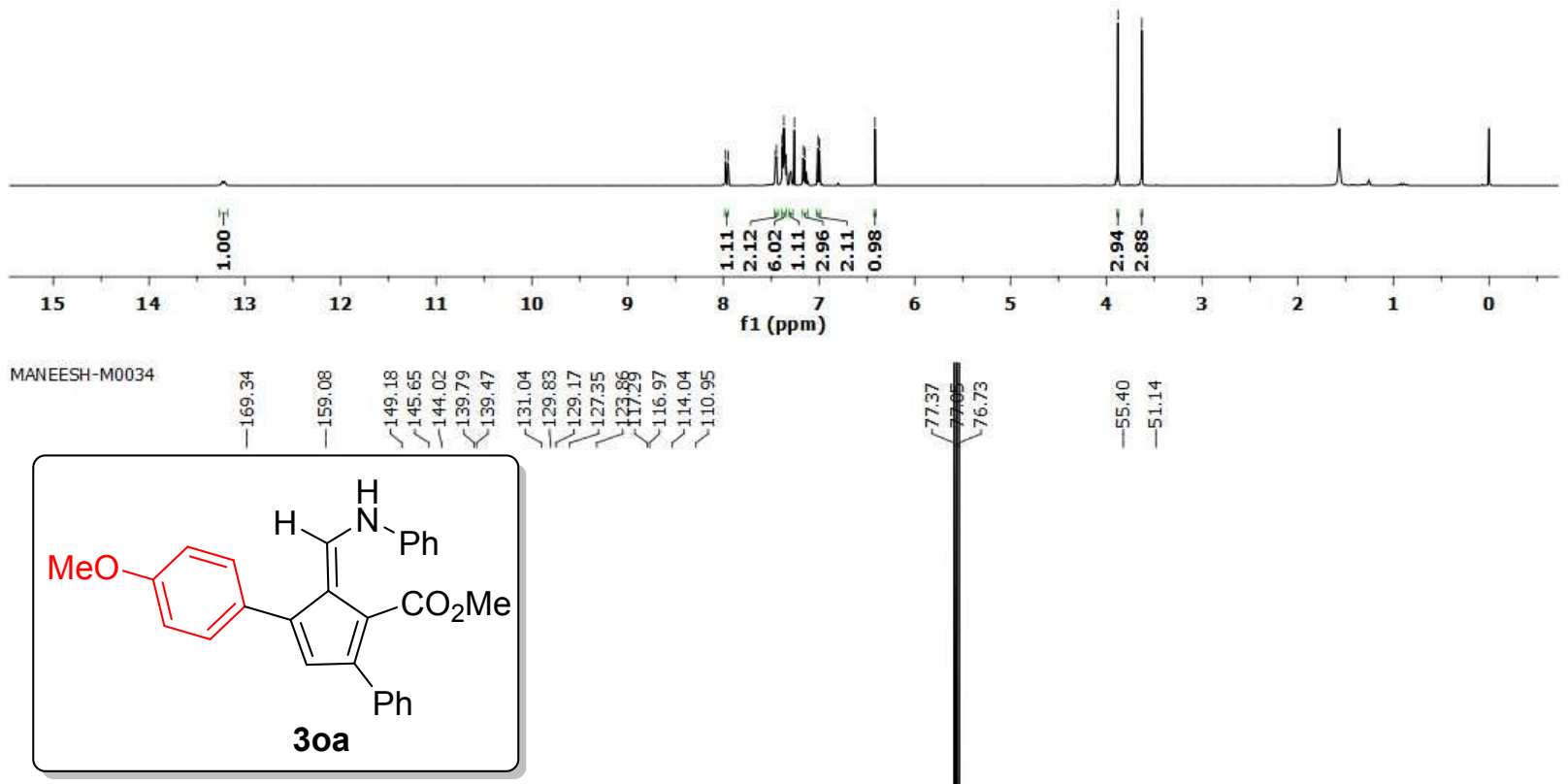

${ }^{13} \mathrm{C} \mathrm{NMR}\left(\mathrm{CDCl}_{3}, 100 \mathrm{MHz}\right)$
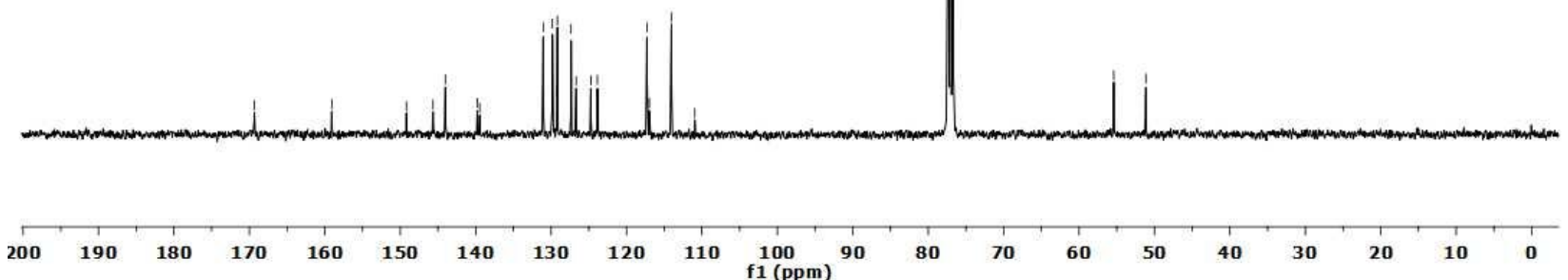

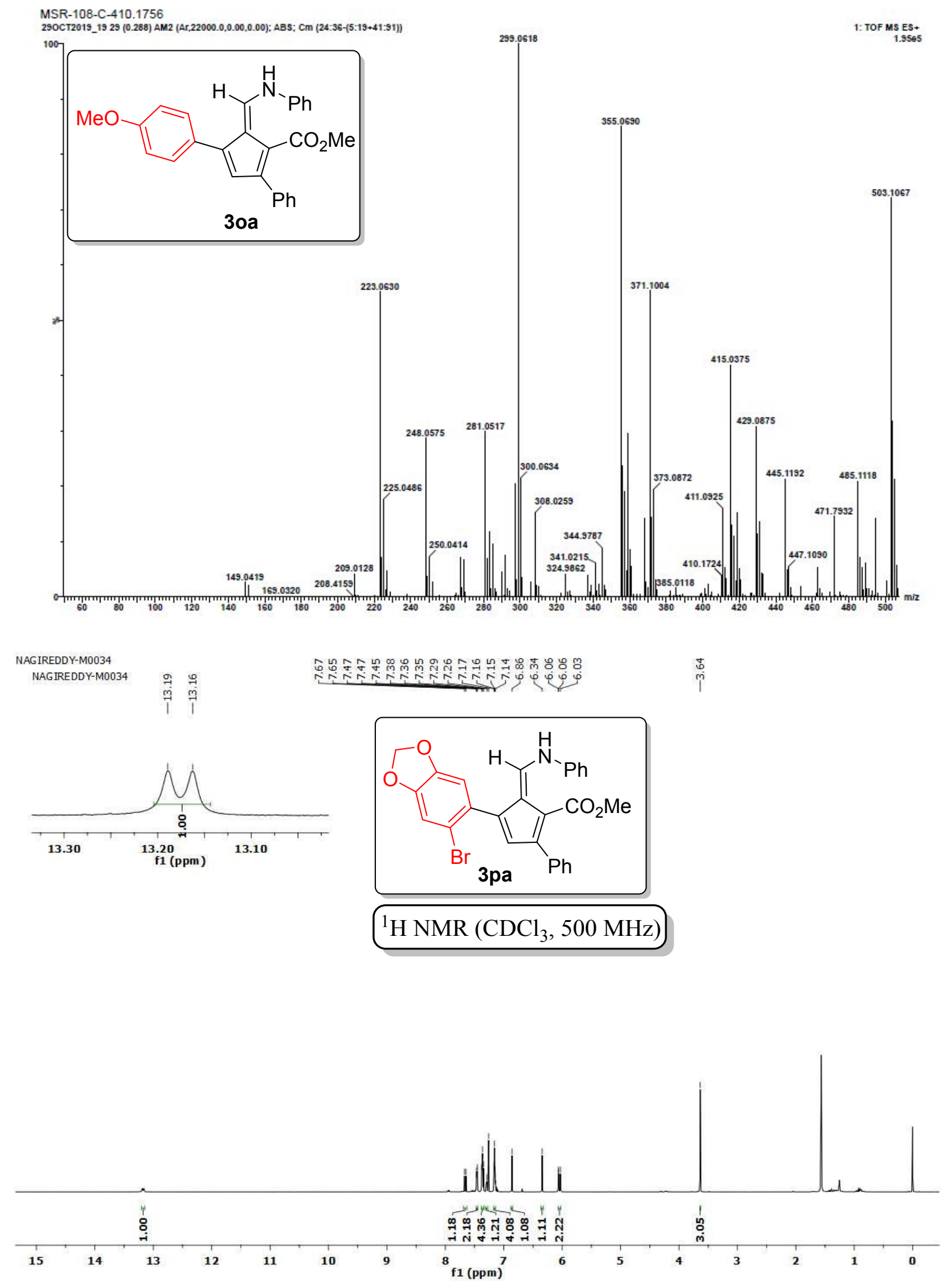

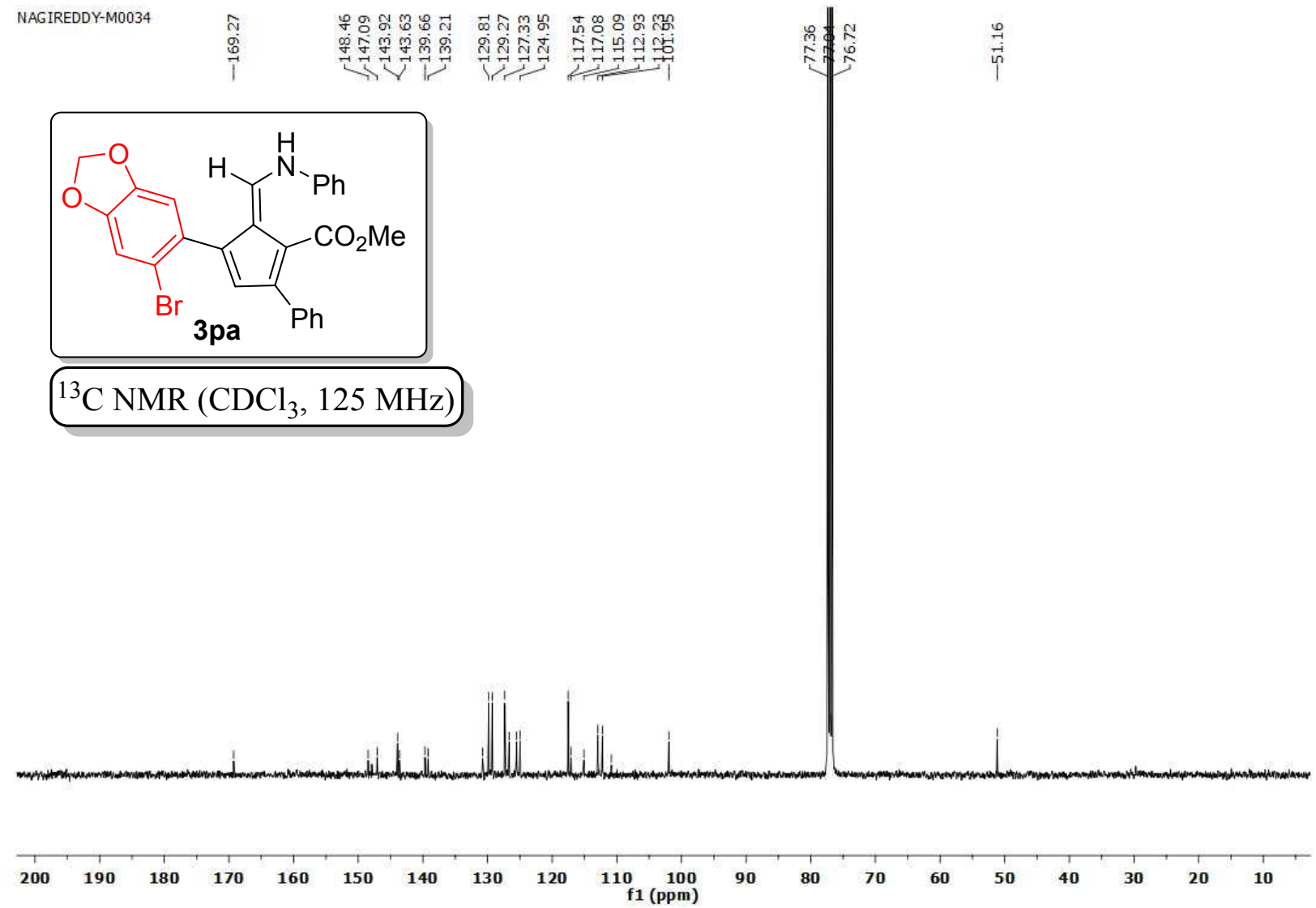

Maddi SRIDHAR REDDY

ThermoScientific EXACTIVE ORBITRAP

Analysed By G Saikrishna

MSR-103-A \#2-14 ${ }^{-} \mathrm{RT}^{-} \mathrm{C}^{-0} 01-0.05^{-} \mathrm{AV}^{-}{ }^{-13^{-}} \mathrm{NL}:{ }^{-} 3.13 \mathrm{E}$

T: FTMS $\{1,1\}+$ p APCl corona Full ms [100.00-2000.00]

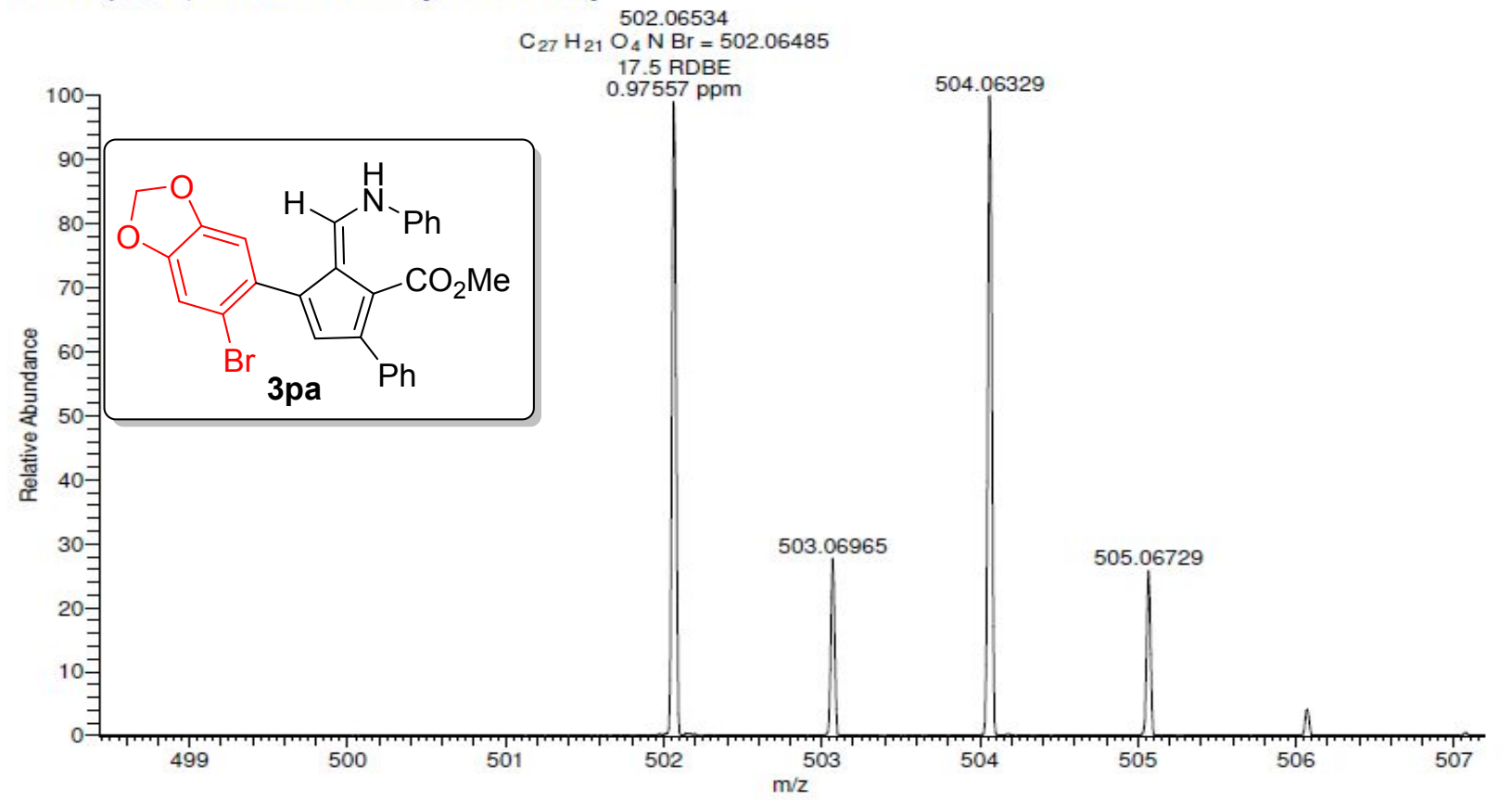



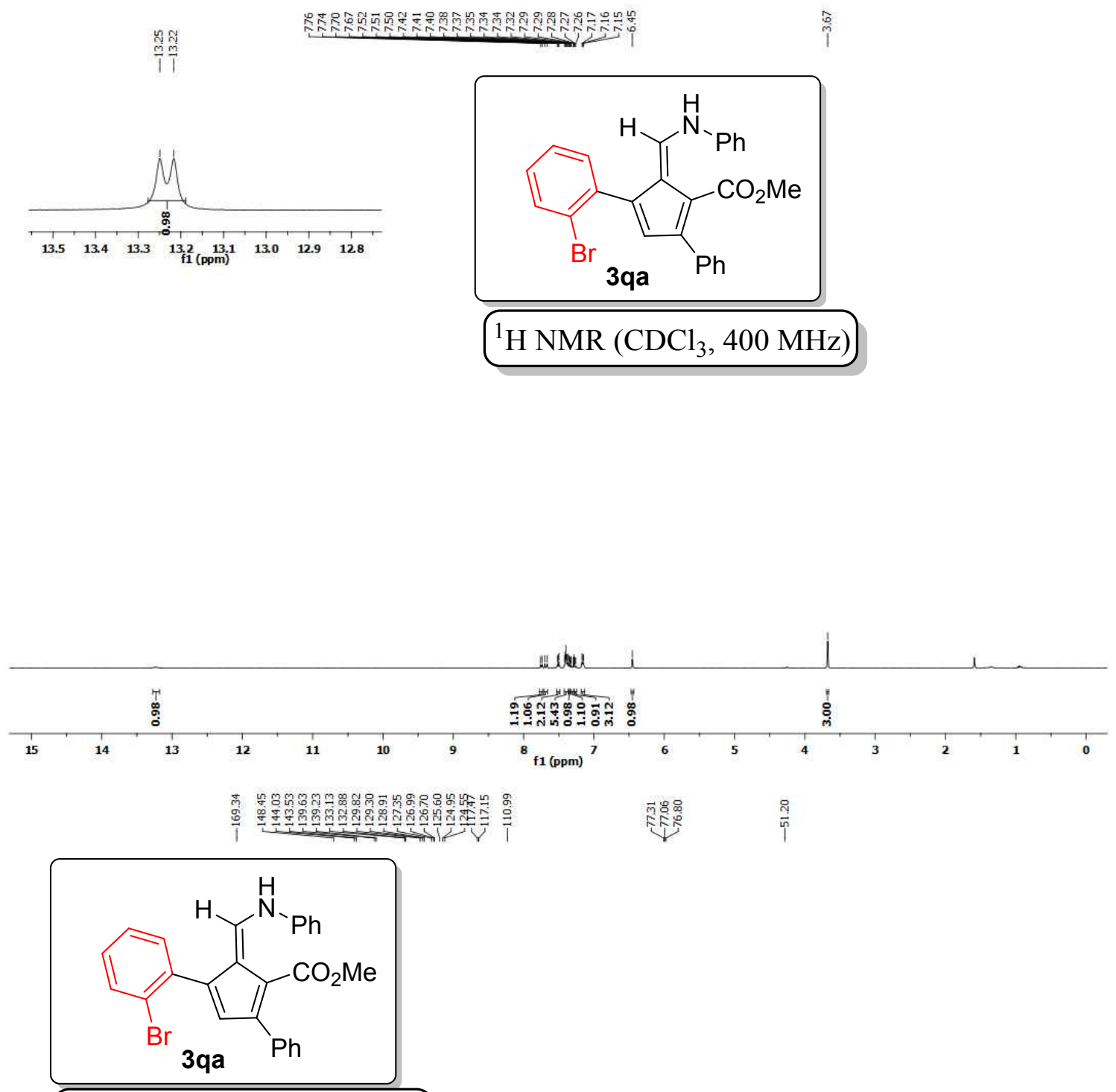

${ }^{13} \mathrm{C} \mathrm{NMR}\left(\mathrm{CDCl}_{3}, 100 \mathrm{MHz}\right)$

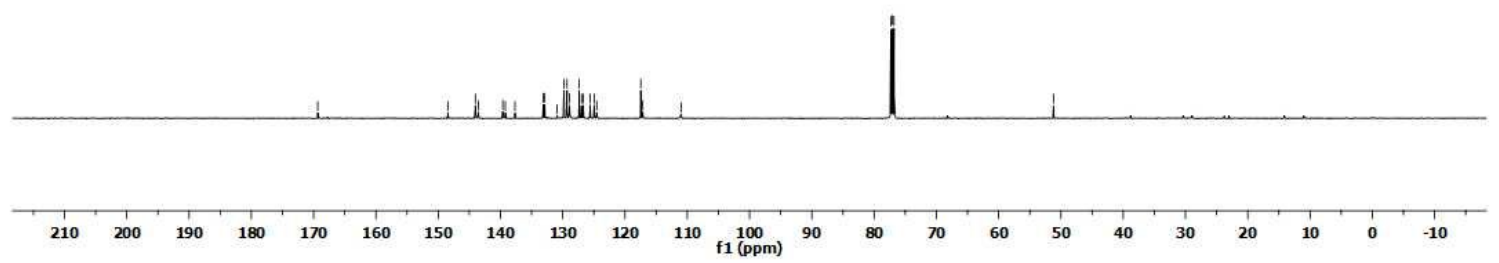



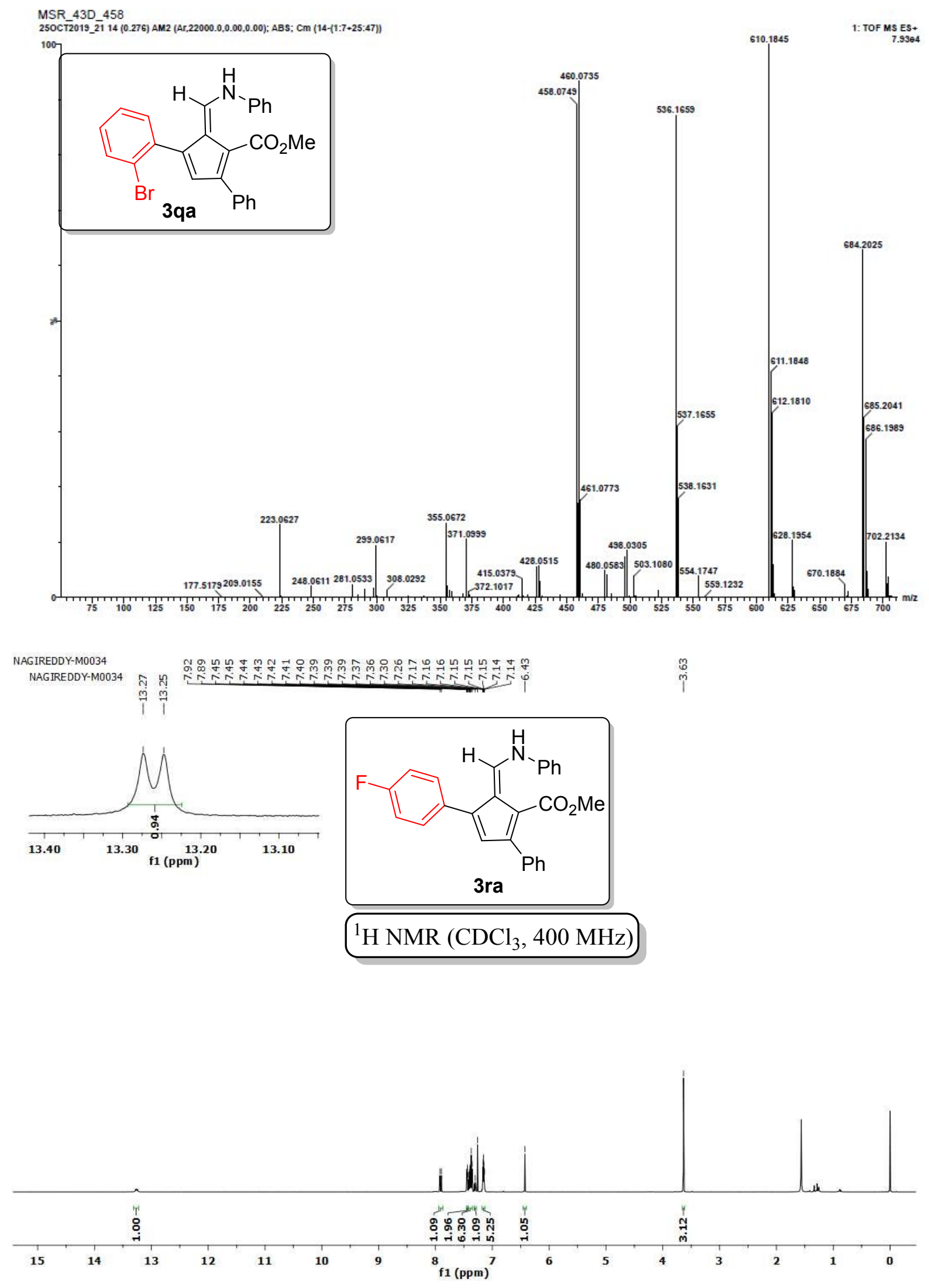

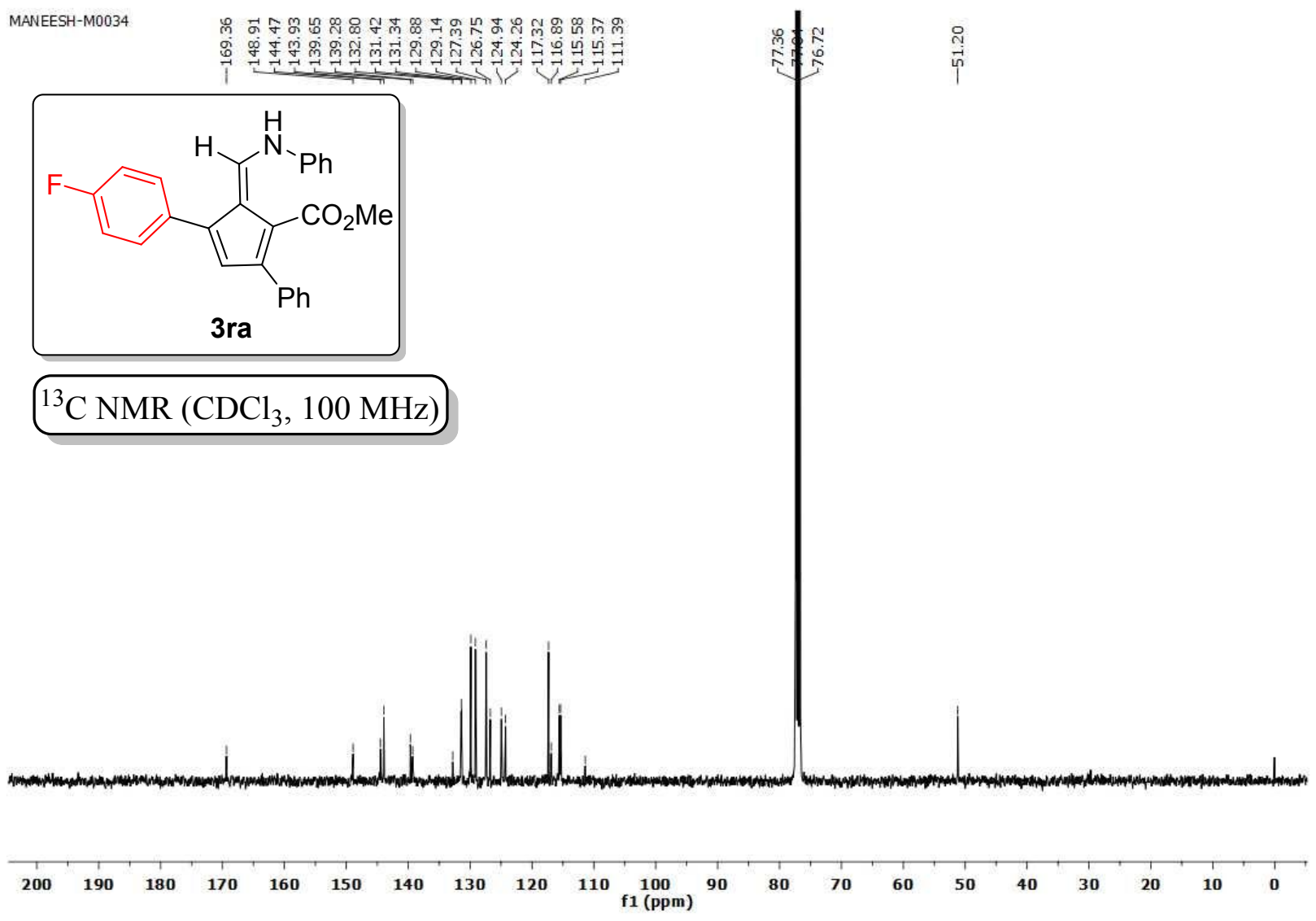

MANEESH-H0011

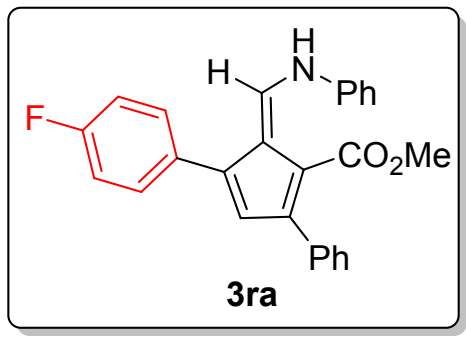

울
i

${ }^{19} \mathrm{~F} \mathrm{NMR}\left(\mathrm{CDCl}_{3}, 377 \mathrm{MHz}\right)$

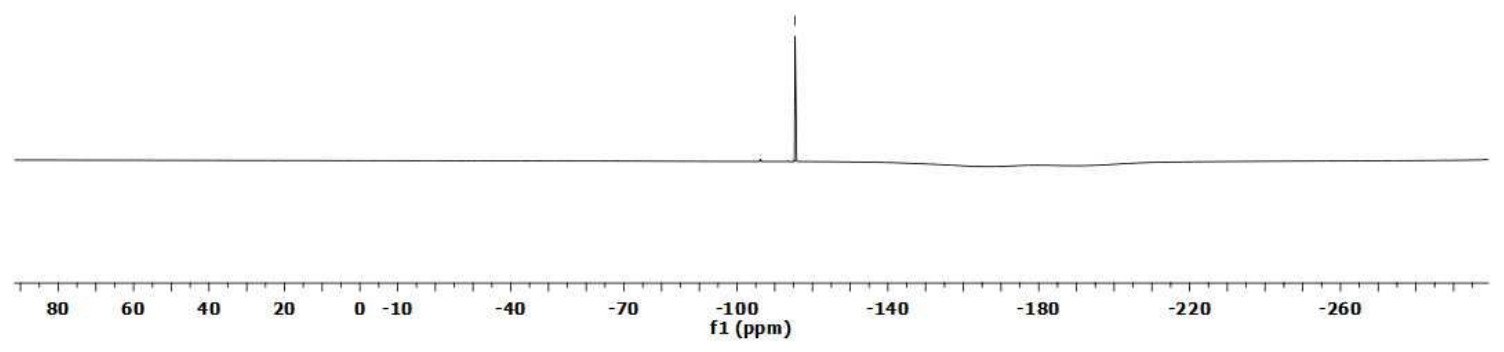




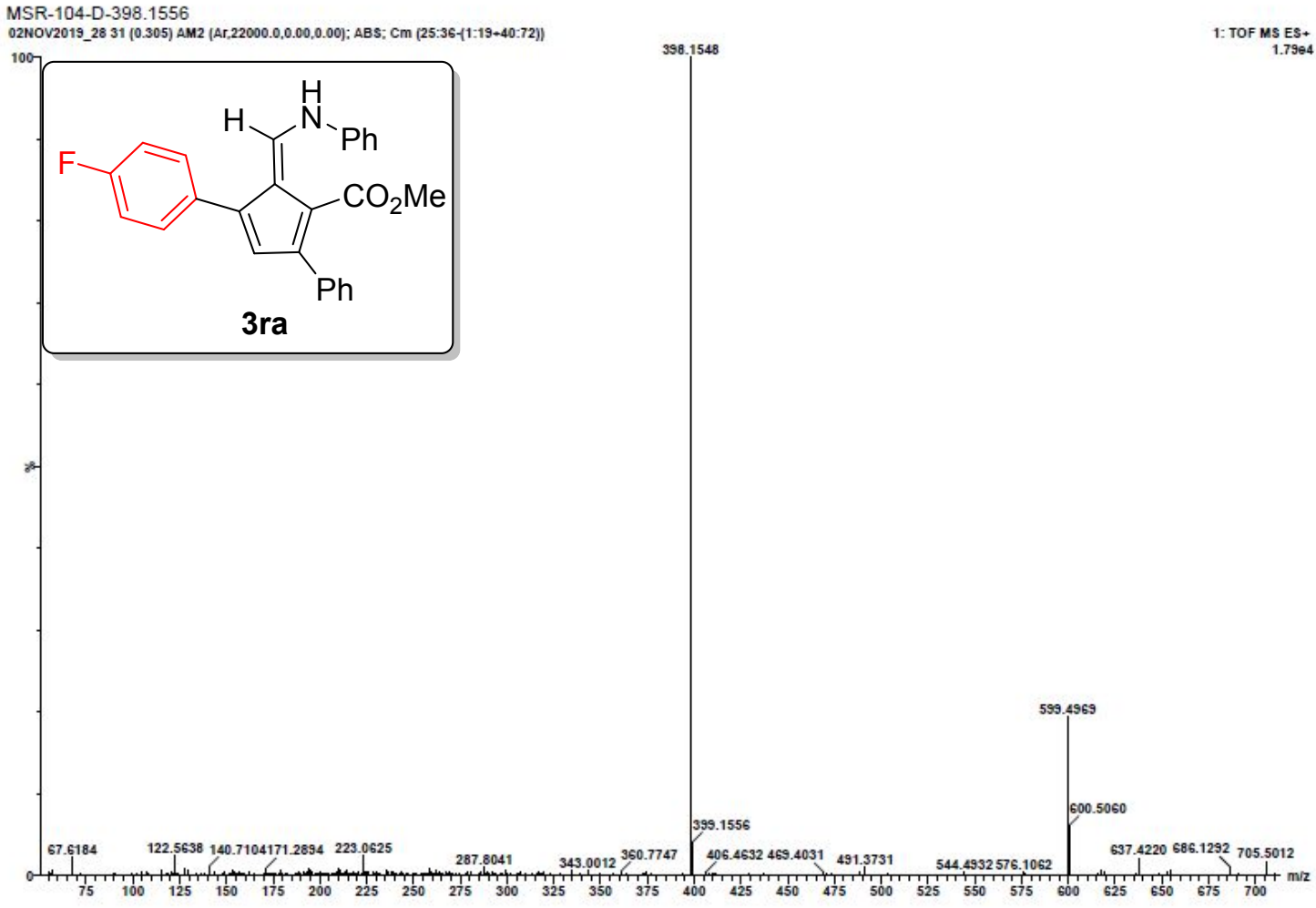




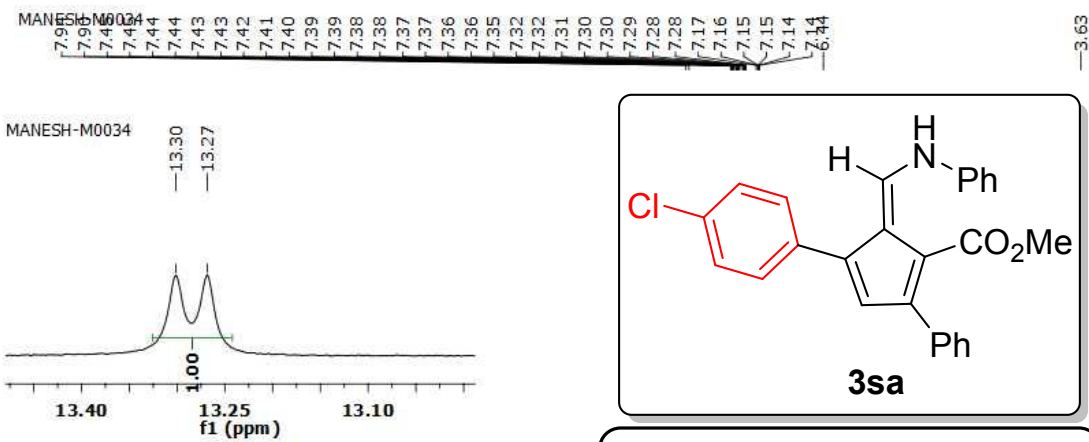

${ }^{1} \mathrm{H}$ NMR $\left(\mathrm{CDCl}_{3}, 400 \mathrm{MHz}\right)$
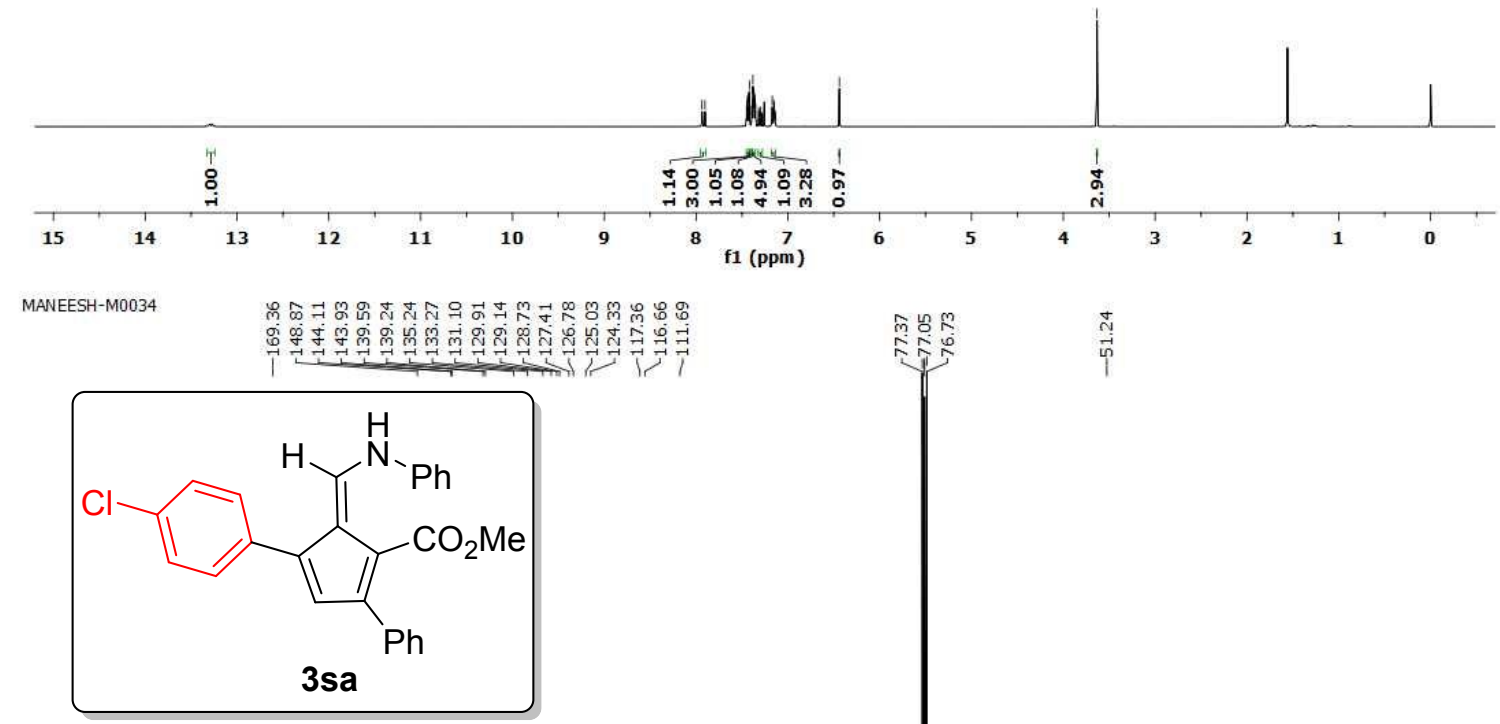

${ }^{13} \mathrm{C} \mathrm{NMR}\left(\mathrm{CDCl}_{3}, 100 \mathrm{MHz}\right)$

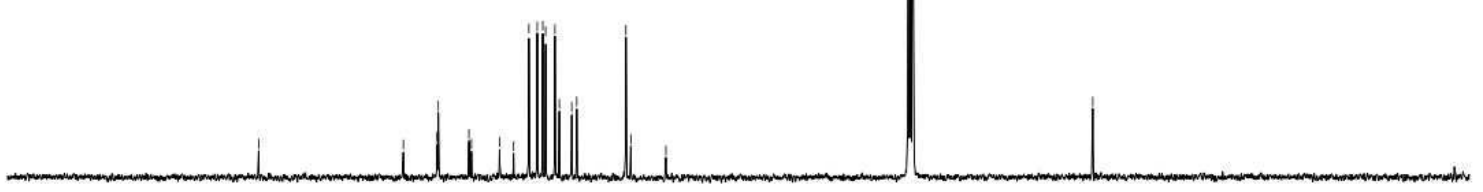

$\begin{array}{llllllllllllllllllllllllllllll}200 & 190 & 180 & 170 & 160 & 150 & 140 & 130 & 120 & 110 & 100 & 90 & 80 & 70 & 60 & 50 & 40 & 30 & 20 & 10 & 0\end{array}$ 
Maddi SRIDHAR REDDY

11/27/2019 4:03:27 PM

ThermoScientific EXACTIVE ORBITRAP

Analysed By G Saikrishna

MSR-106-A \#2-14 ${ }^{-} \mathrm{RT}: \mathrm{C}^{-} 0.01-0.05^{-} \mathrm{AV}^{-}{ }^{-1}{ }^{-} \mathrm{NL}:{ }^{-} 2.13 \mathrm{E} 8$

T: FTMS $\{1,1\}+p$ APCl corona Full ms [100.00-2000.00]

414.12581

$\mathrm{C}_{26} \mathrm{H}_{21} \mathrm{O}_{2} \mathrm{~N} \mathrm{Cl}=414.12553$

16.5 RDBE

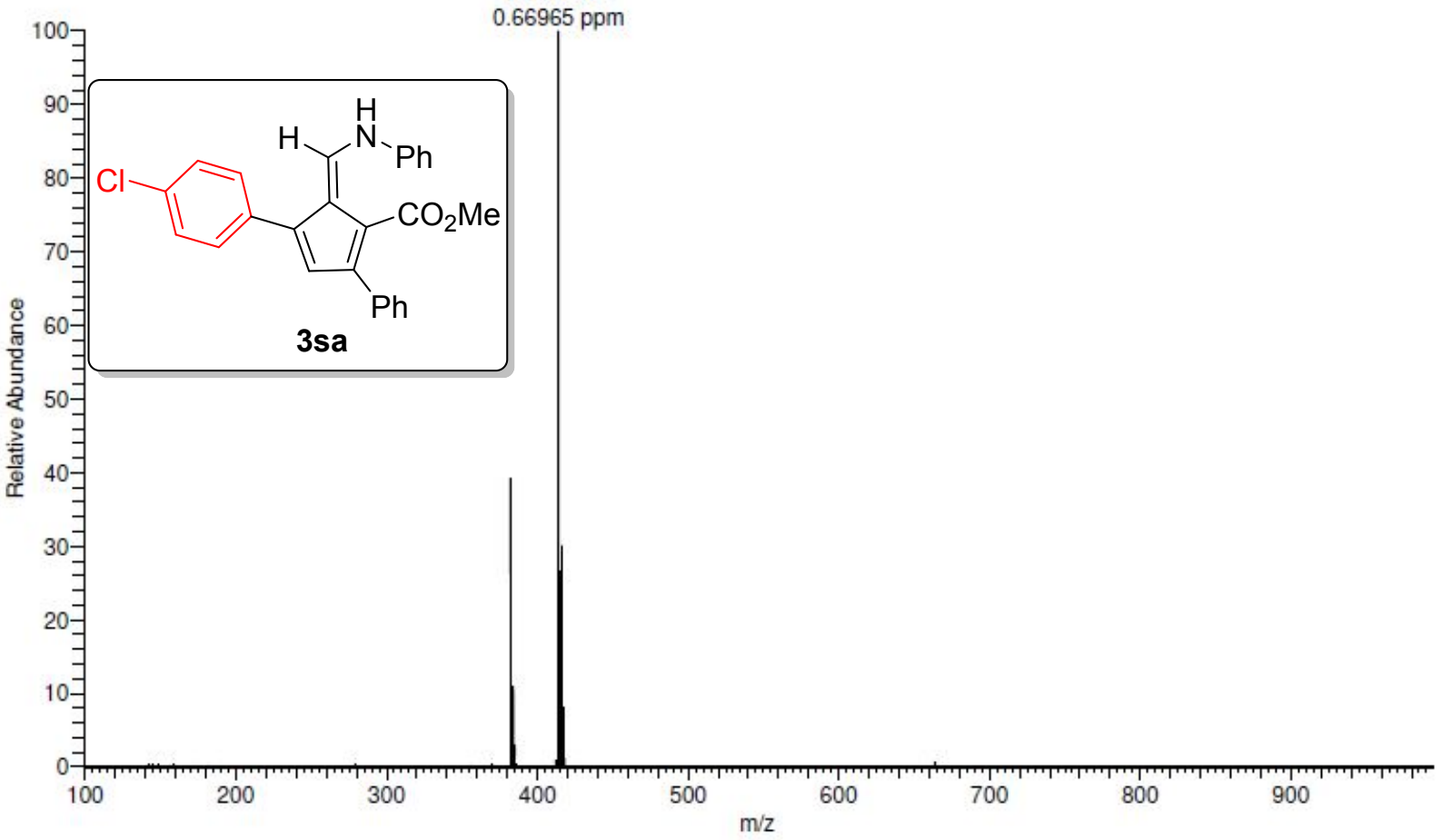

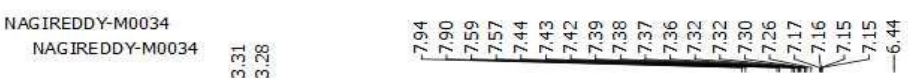

$\stackrel{\circ}{\varphi}$

7
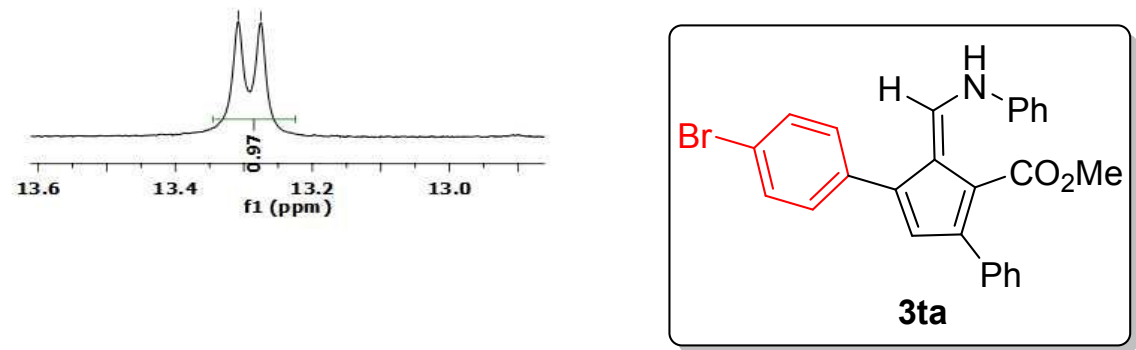

${ }^{1} \mathrm{H} \mathrm{NMR}\left(\mathrm{CDCl}_{3}, 400 \mathrm{MHz}\right)$

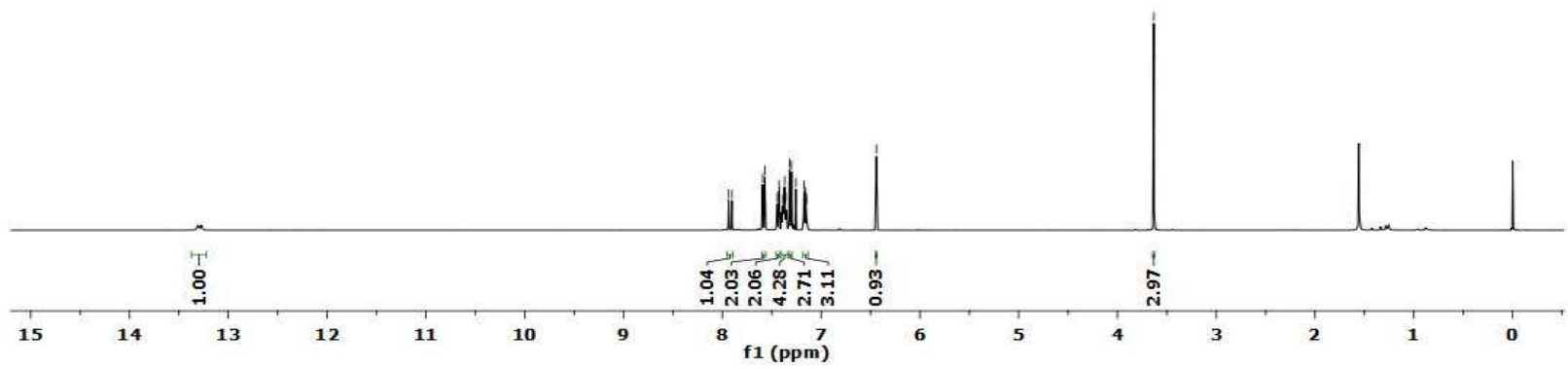



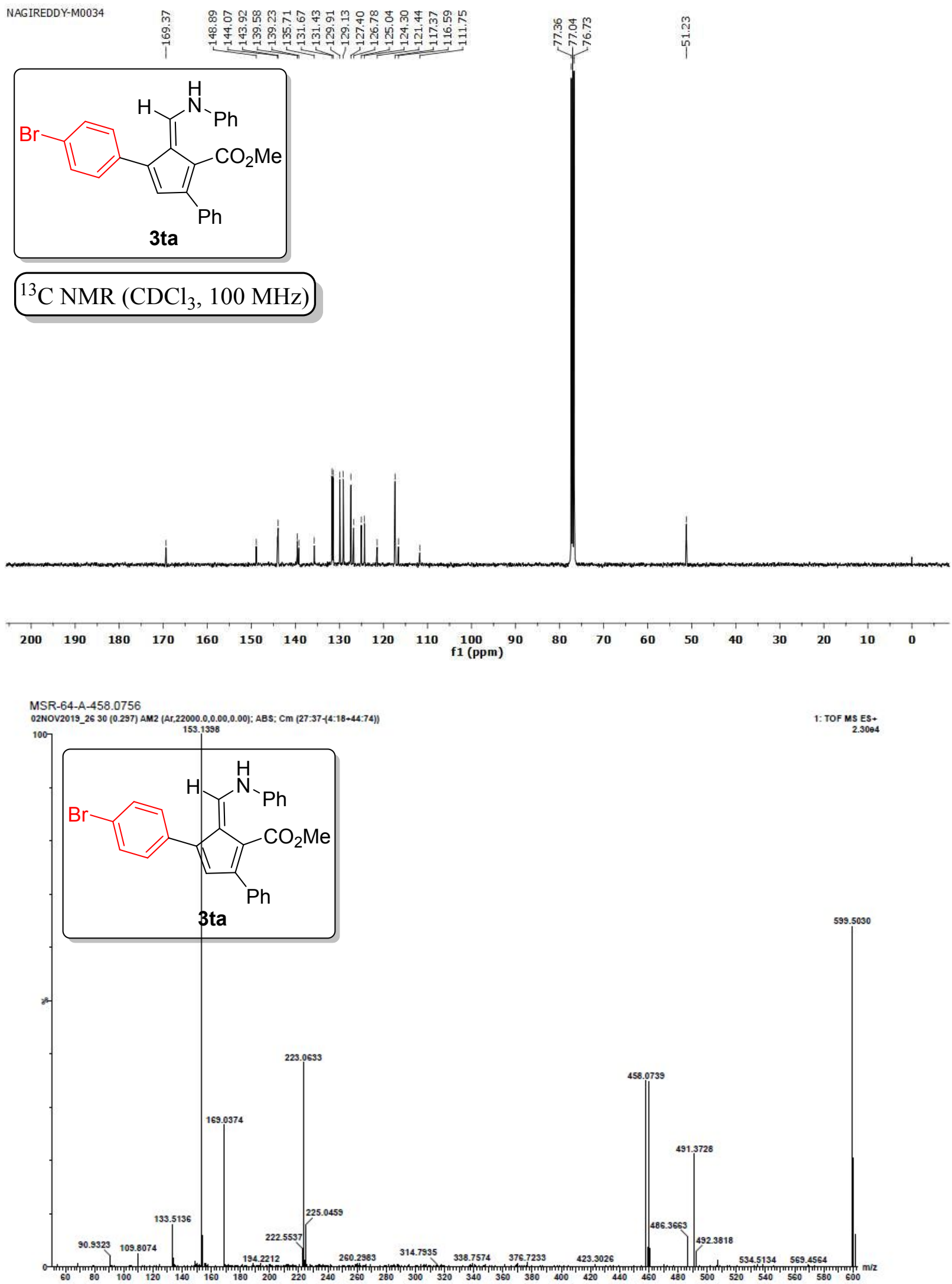

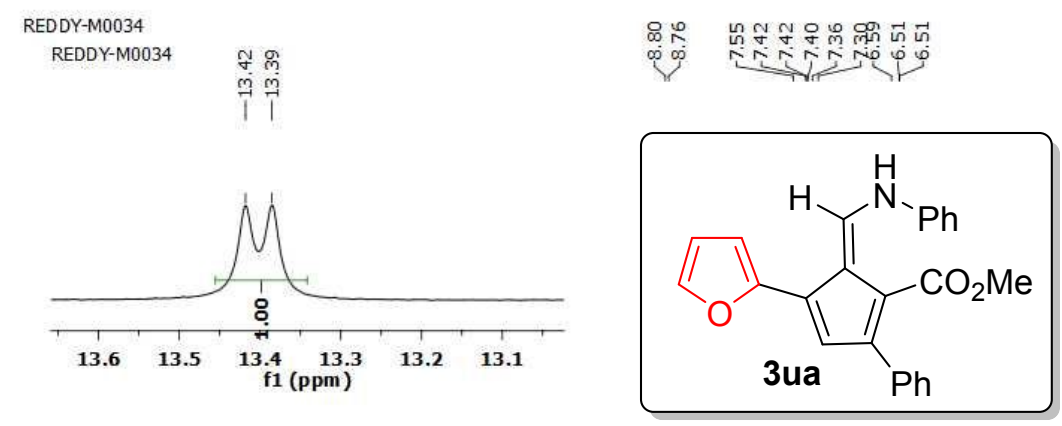

${ }^{1} \mathrm{H} \mathrm{NMR}\left(\mathrm{CDCl}_{3}, 500 \mathrm{MHz}\right)$
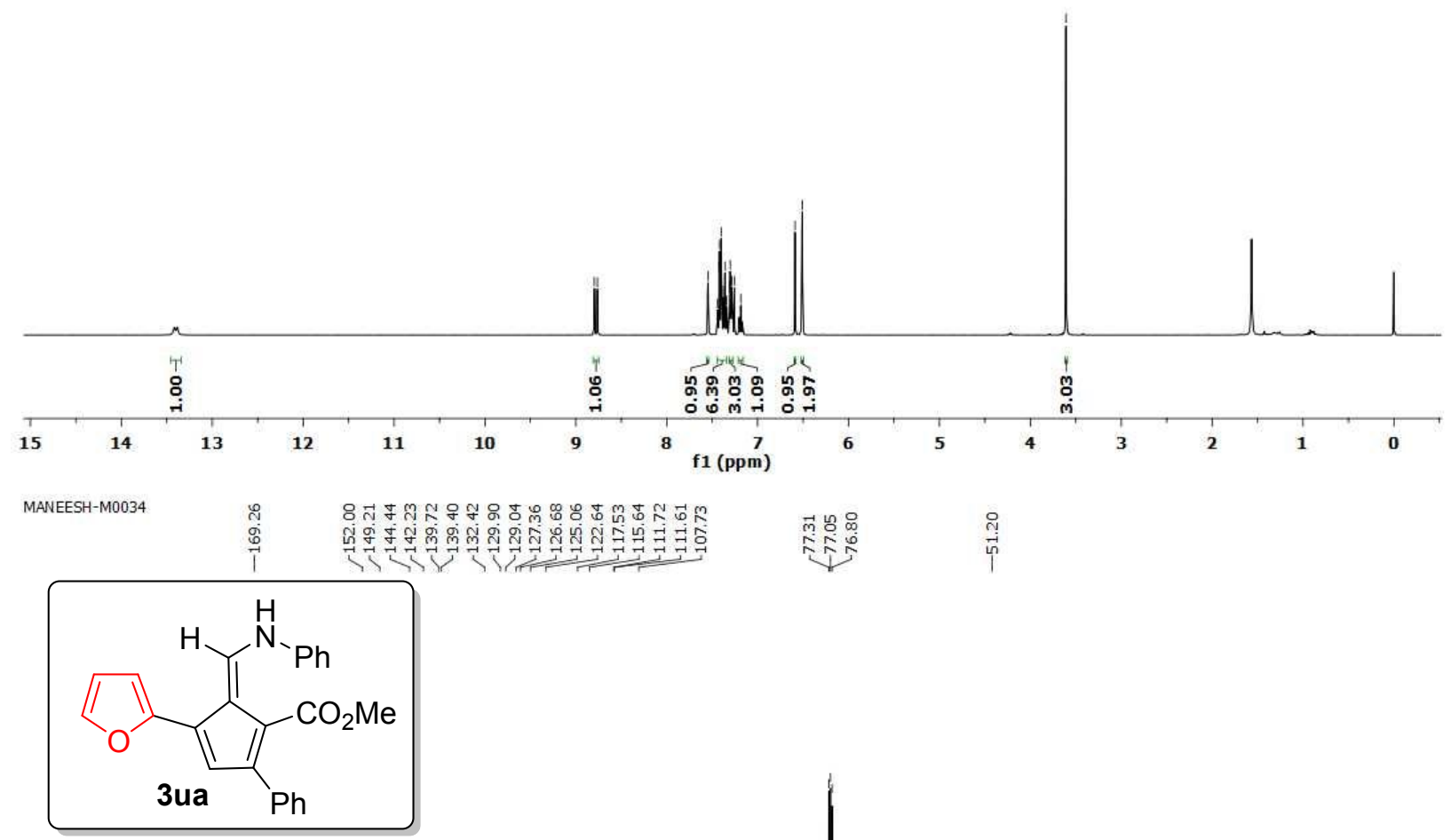

${ }^{13} \mathrm{C} \mathrm{NMR}\left(\mathrm{CDCl}_{3}, 125 \mathrm{MHz}\right)$
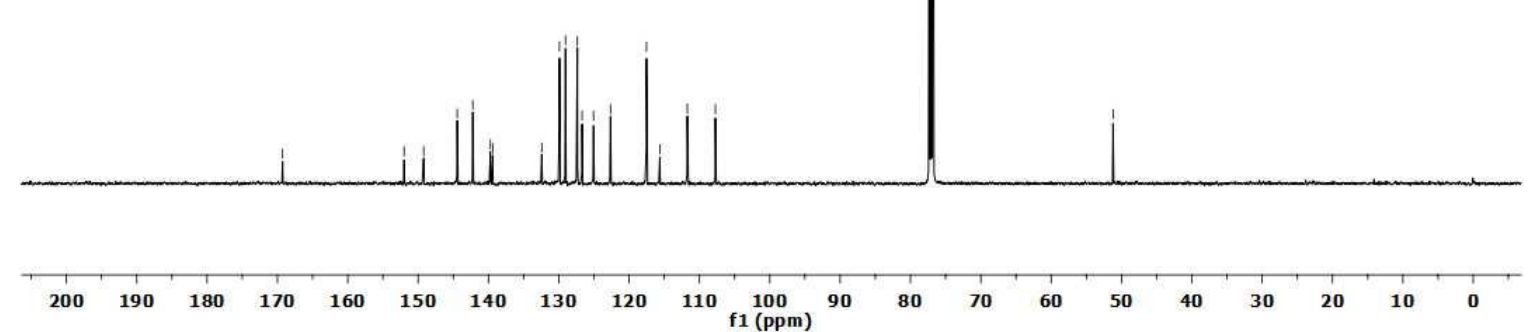

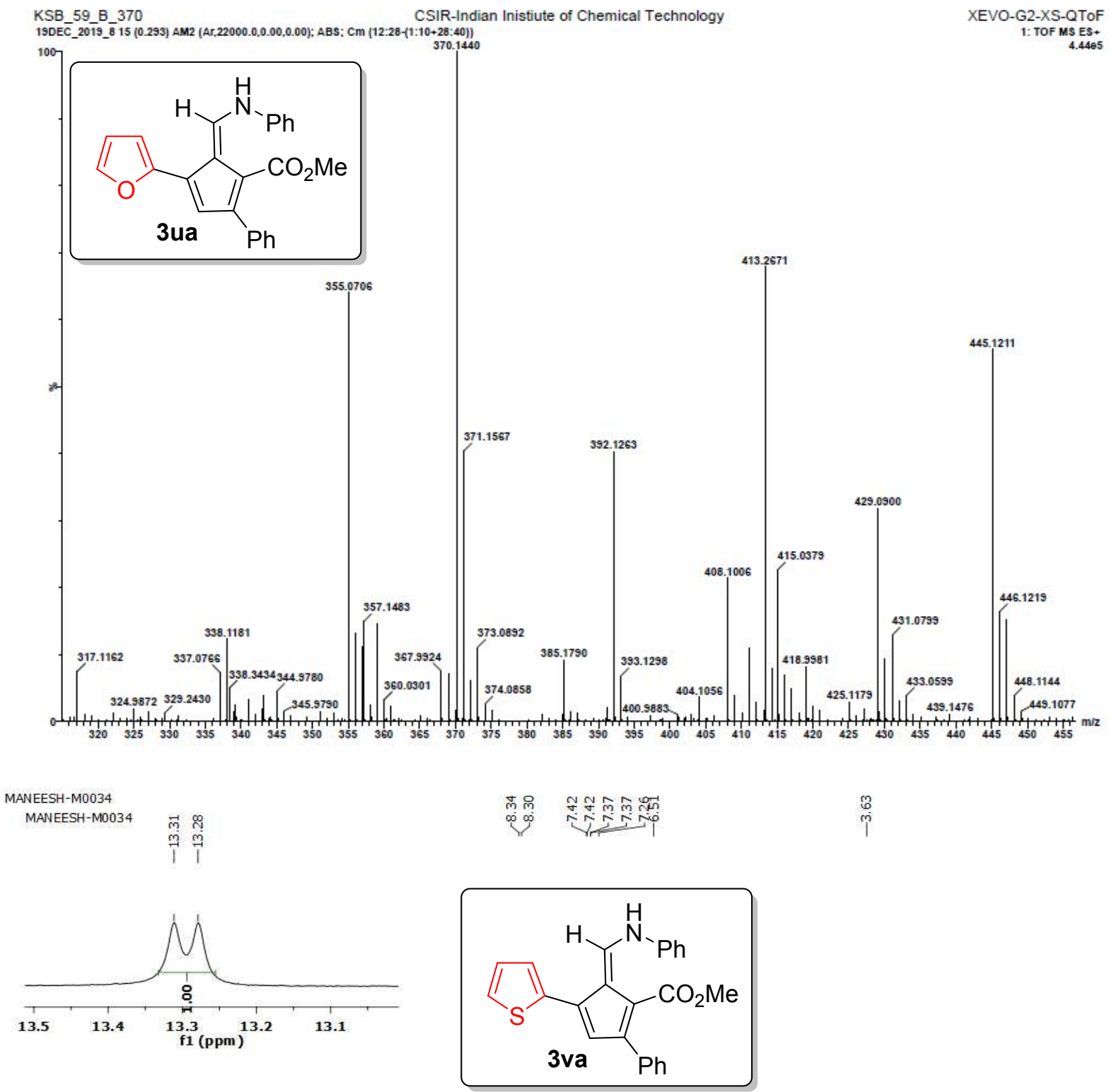

${ }^{1} \mathrm{H} \mathrm{NMR}\left(\mathrm{CDCl}_{3}, 500 \mathrm{MHz}\right)$

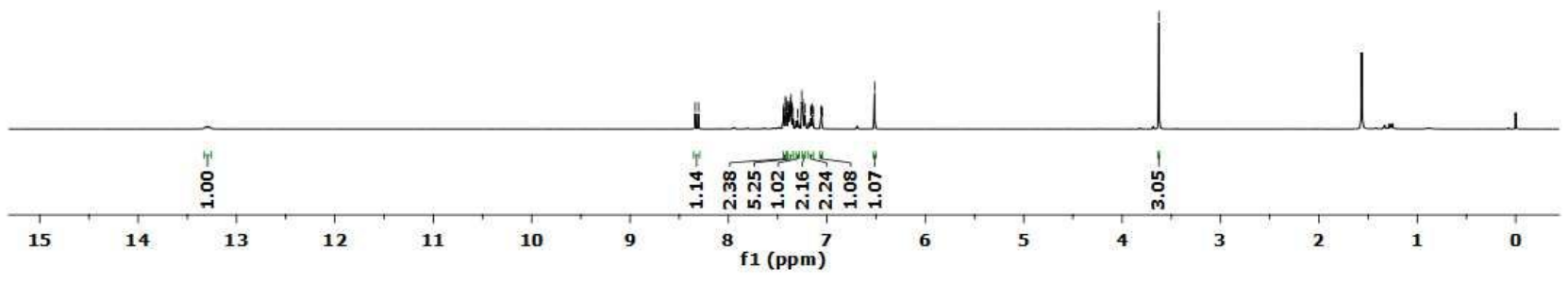




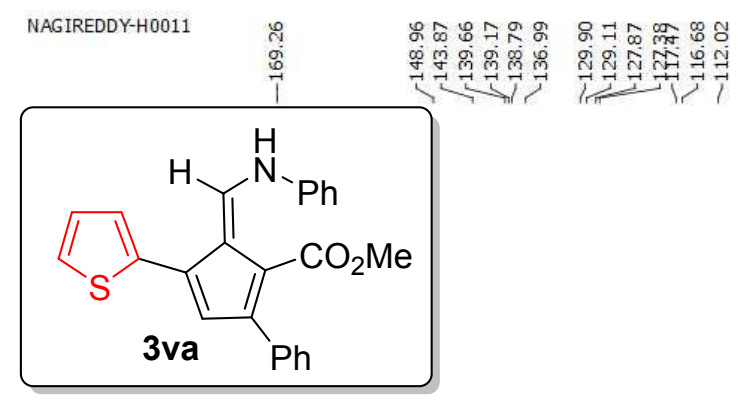

${ }^{13} \mathrm{C} \mathrm{NMR}\left(\mathrm{CDCl}_{3}, 125 \mathrm{MHz}\right)$
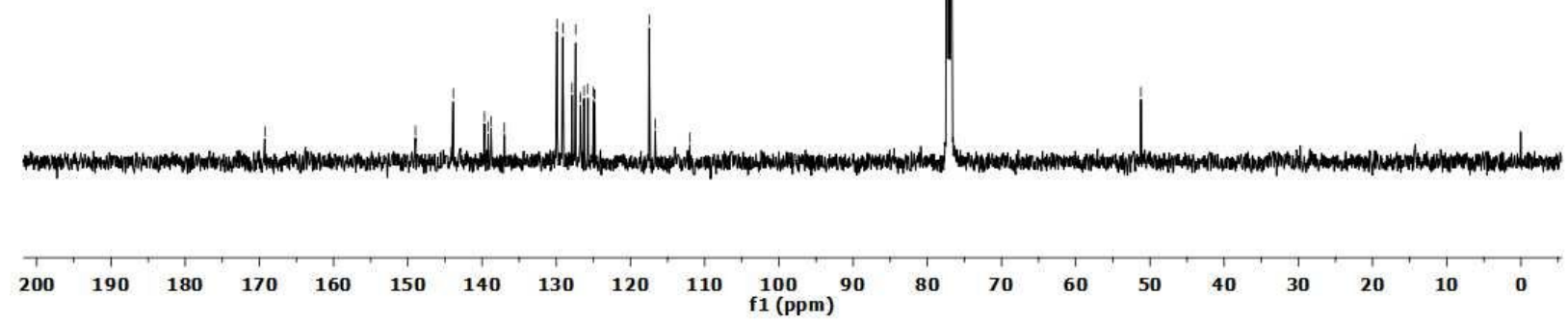

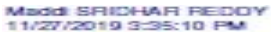

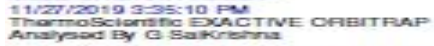

MSR-7S-A \#1-12-RT:-0.01-0.05-AV:-12- ${ }^{-}$NUL =-1.9BE8

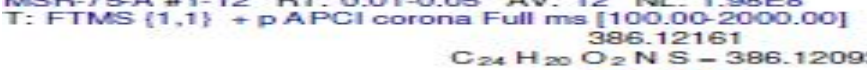

$$
C_{24} H_{20} \mathrm{O}=\mathrm{N} S-38
$$

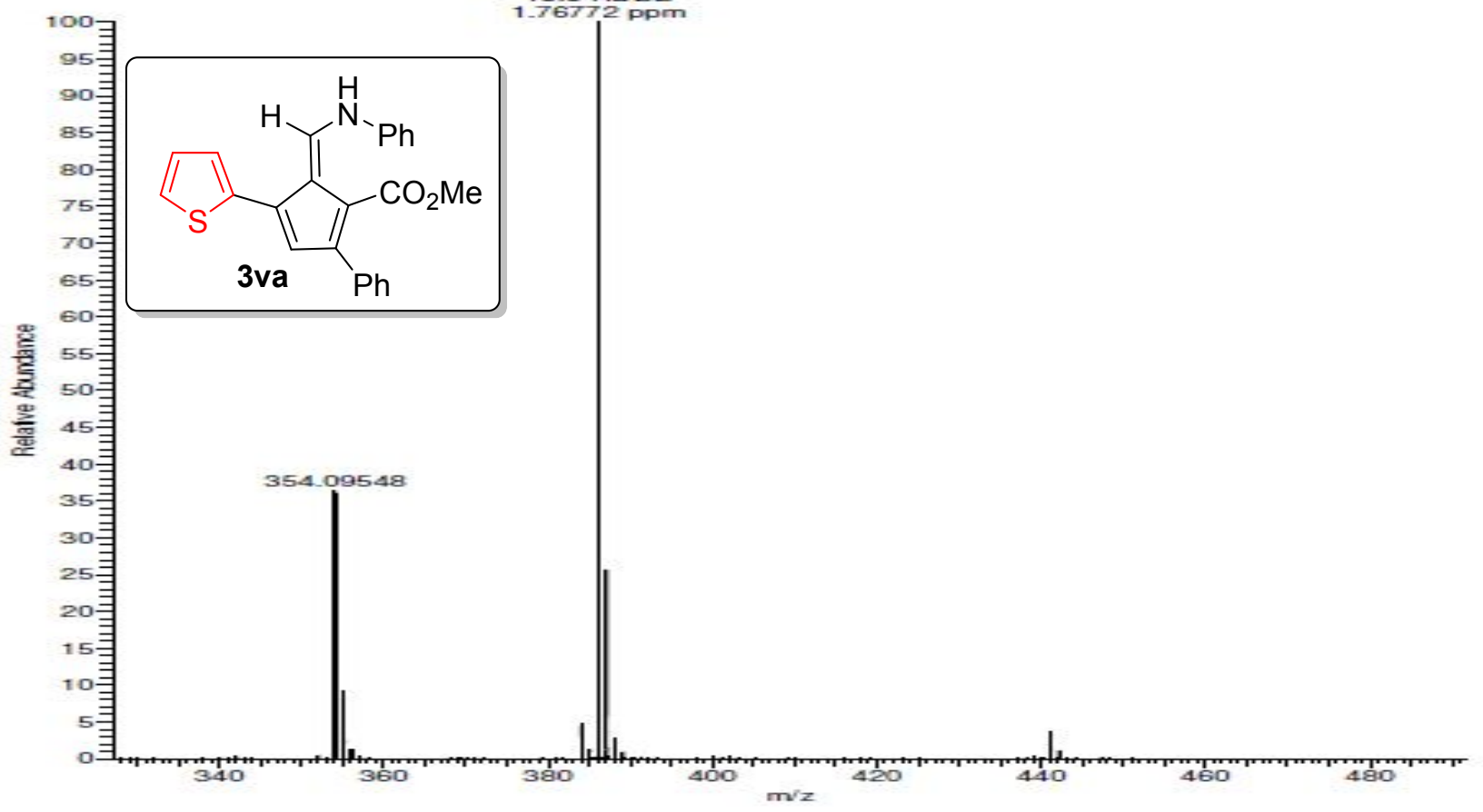



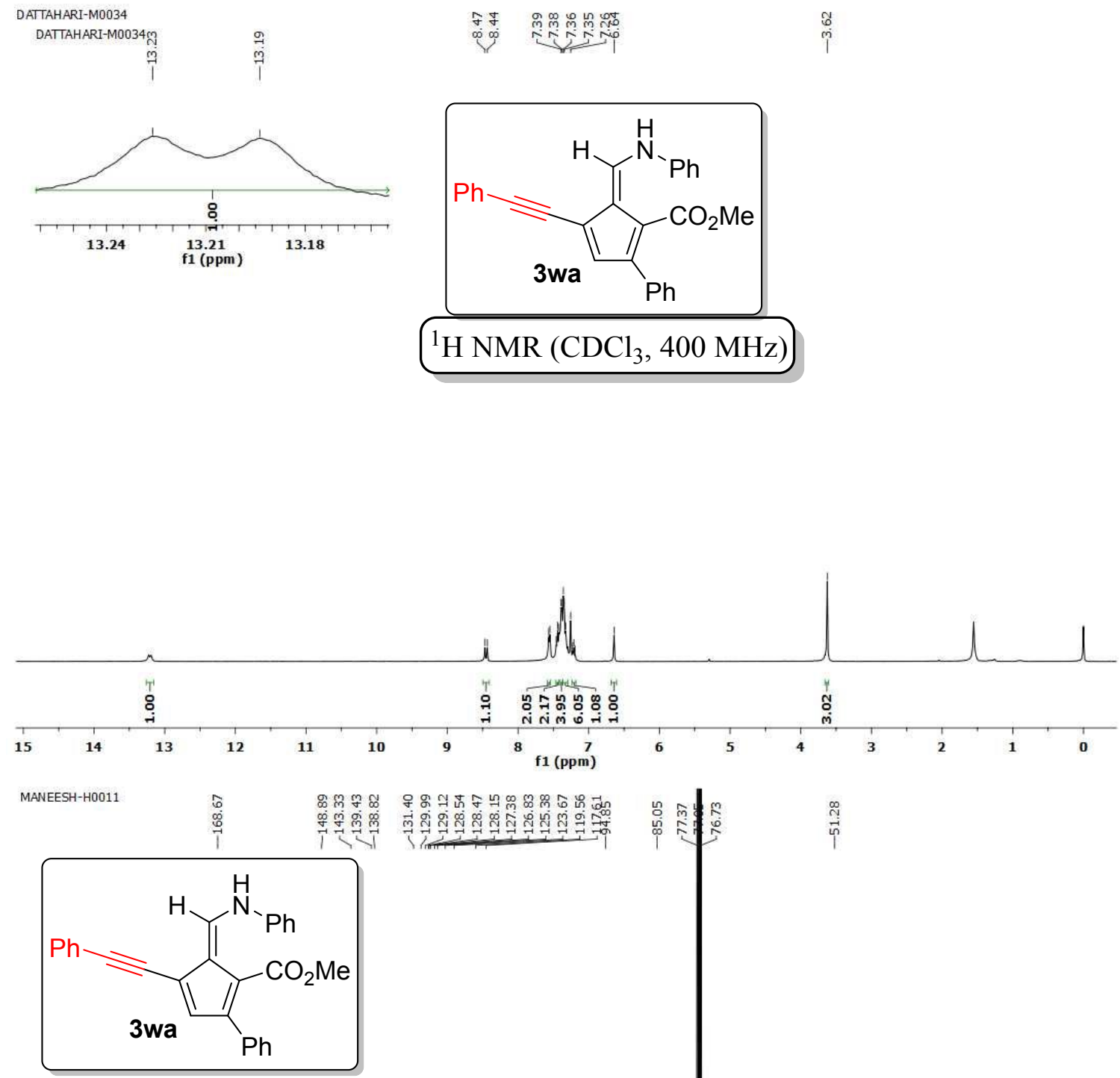

${ }^{13} \mathrm{C} \mathrm{NMR}\left(\mathrm{CDCl}_{3}, 100 \mathrm{MHz}\right)$
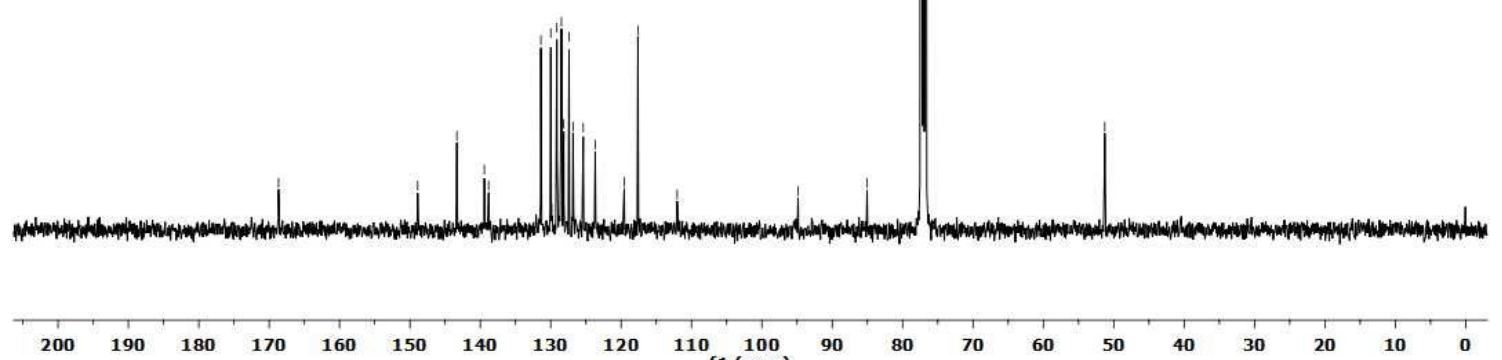


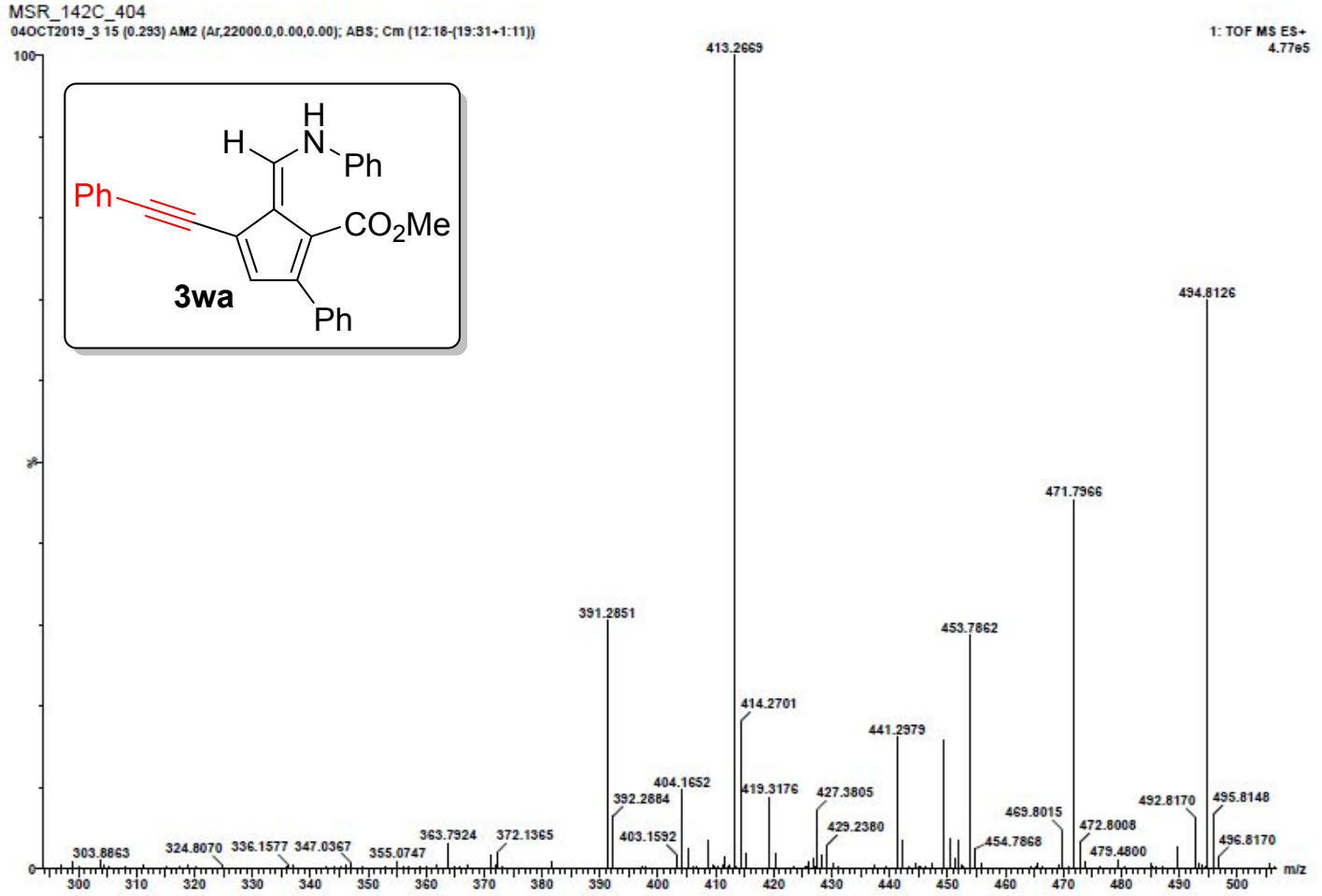



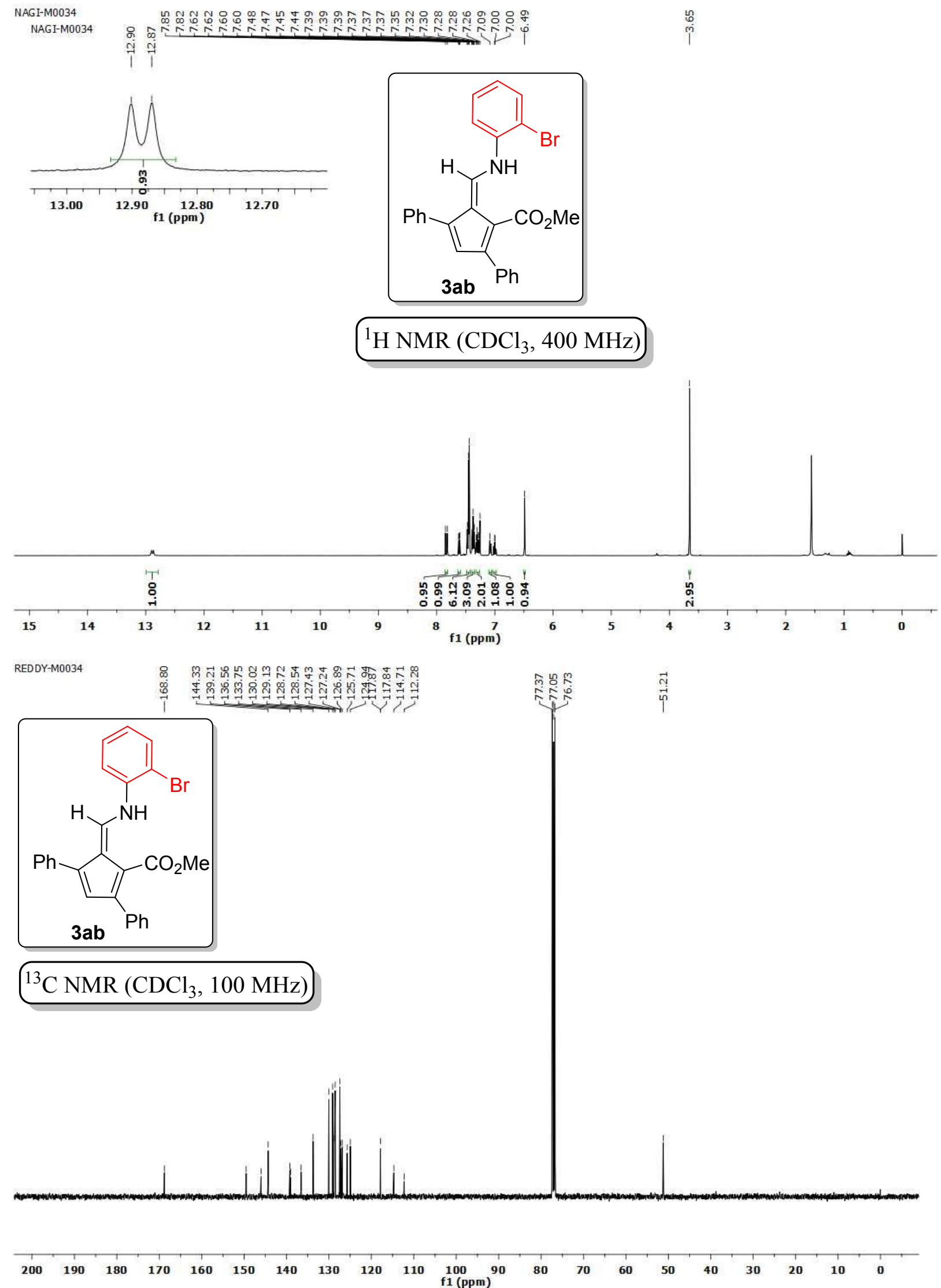

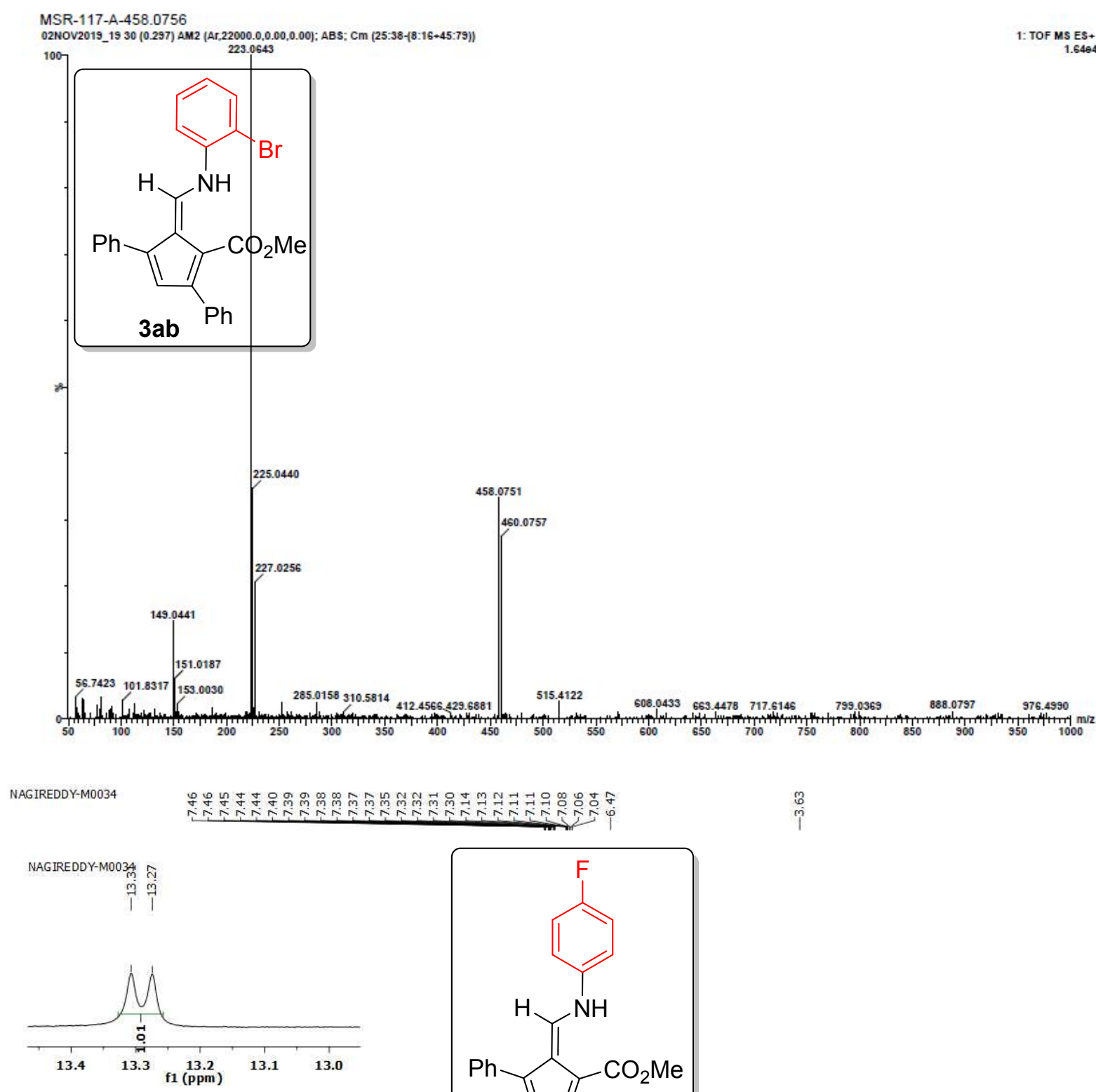

$\stackrel{\substack{0 \\ i}}{\oplus}$

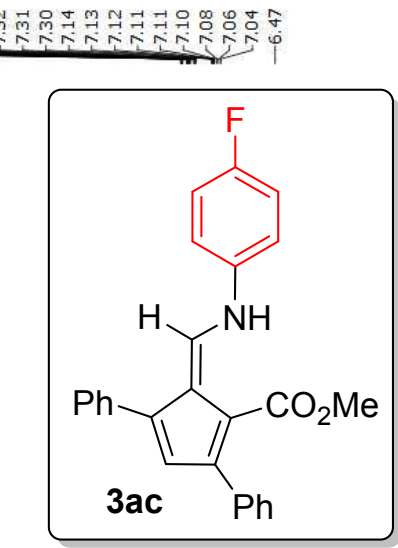

${ }^{1} \mathrm{H} \mathrm{NMR}\left(\mathrm{CDCl}_{3}, 400 \mathrm{MHz}\right)$

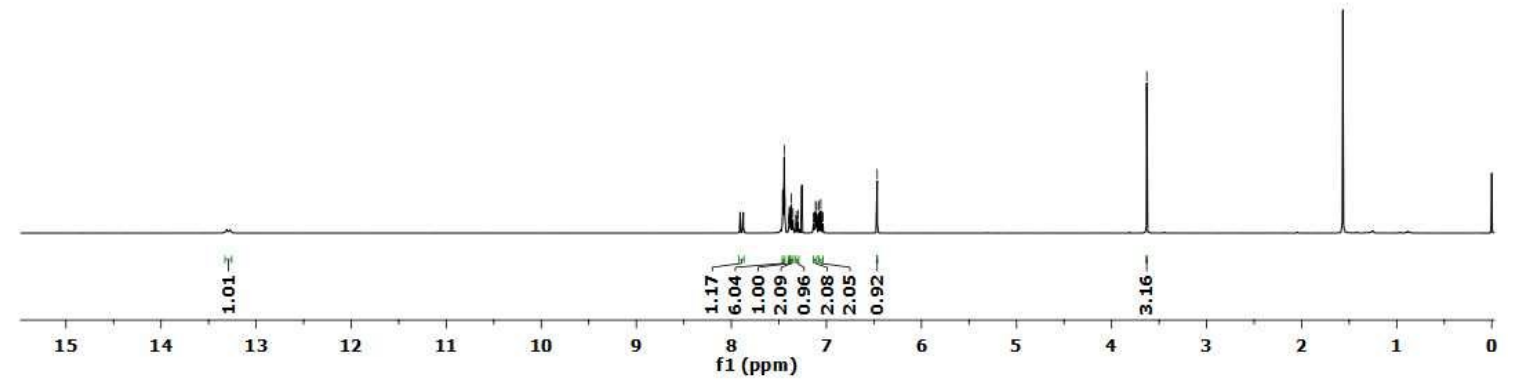



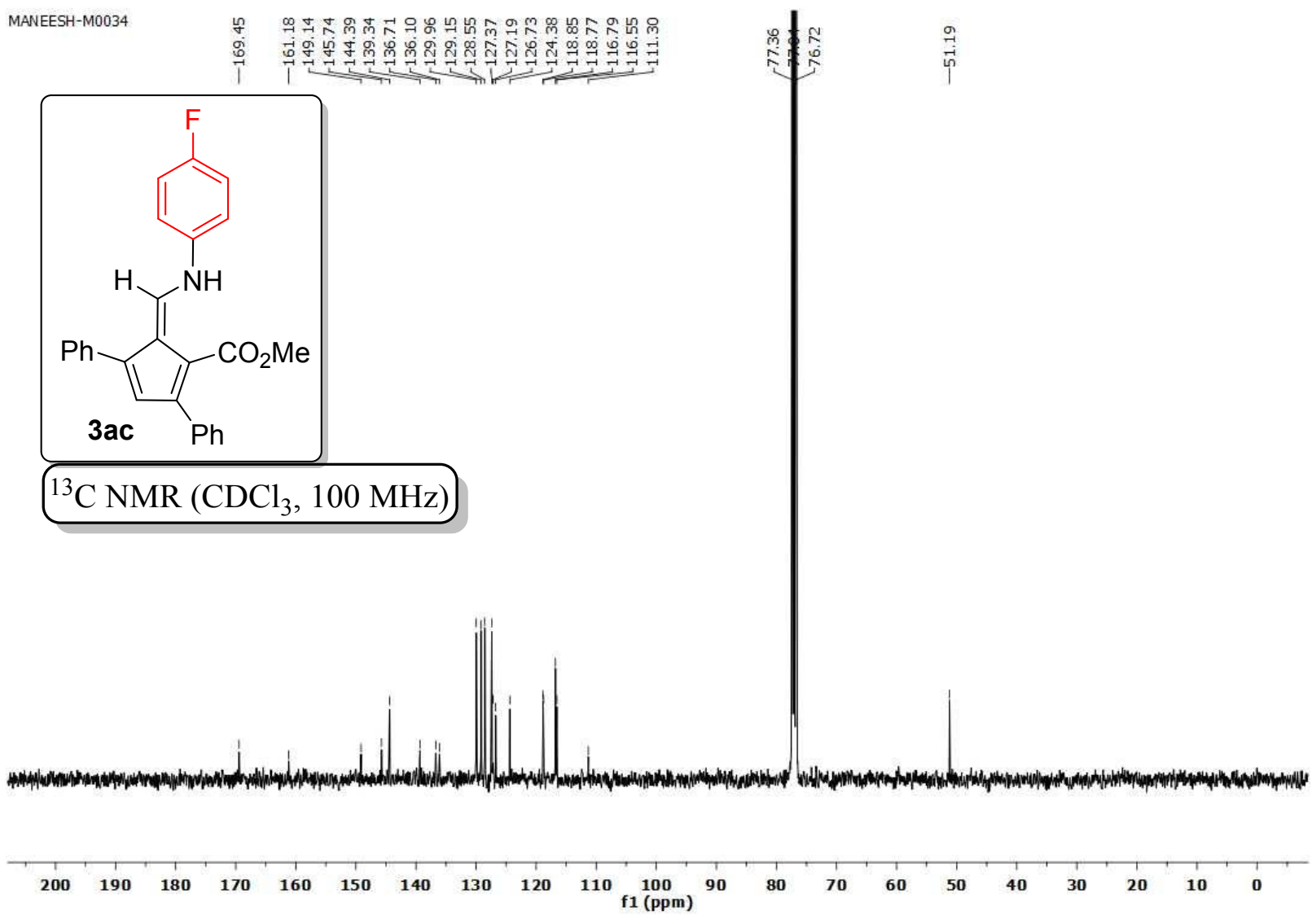

MANEESH-M0034

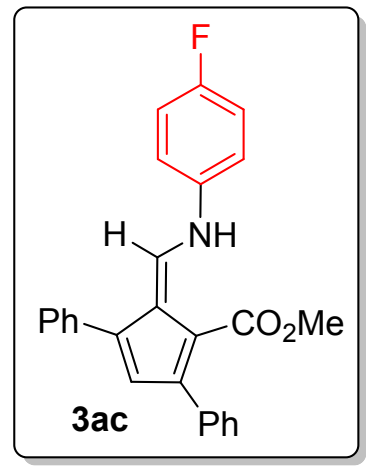

${ }^{19} \mathrm{~F} \mathrm{NMR}\left(\mathrm{CDCl}_{3}, 377 \mathrm{MHz}\right)$

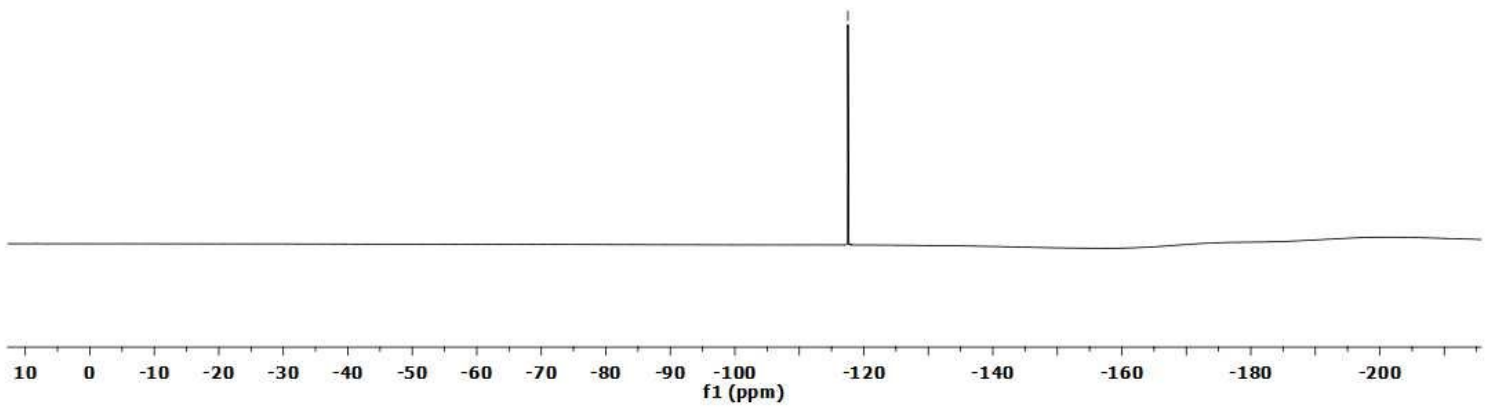




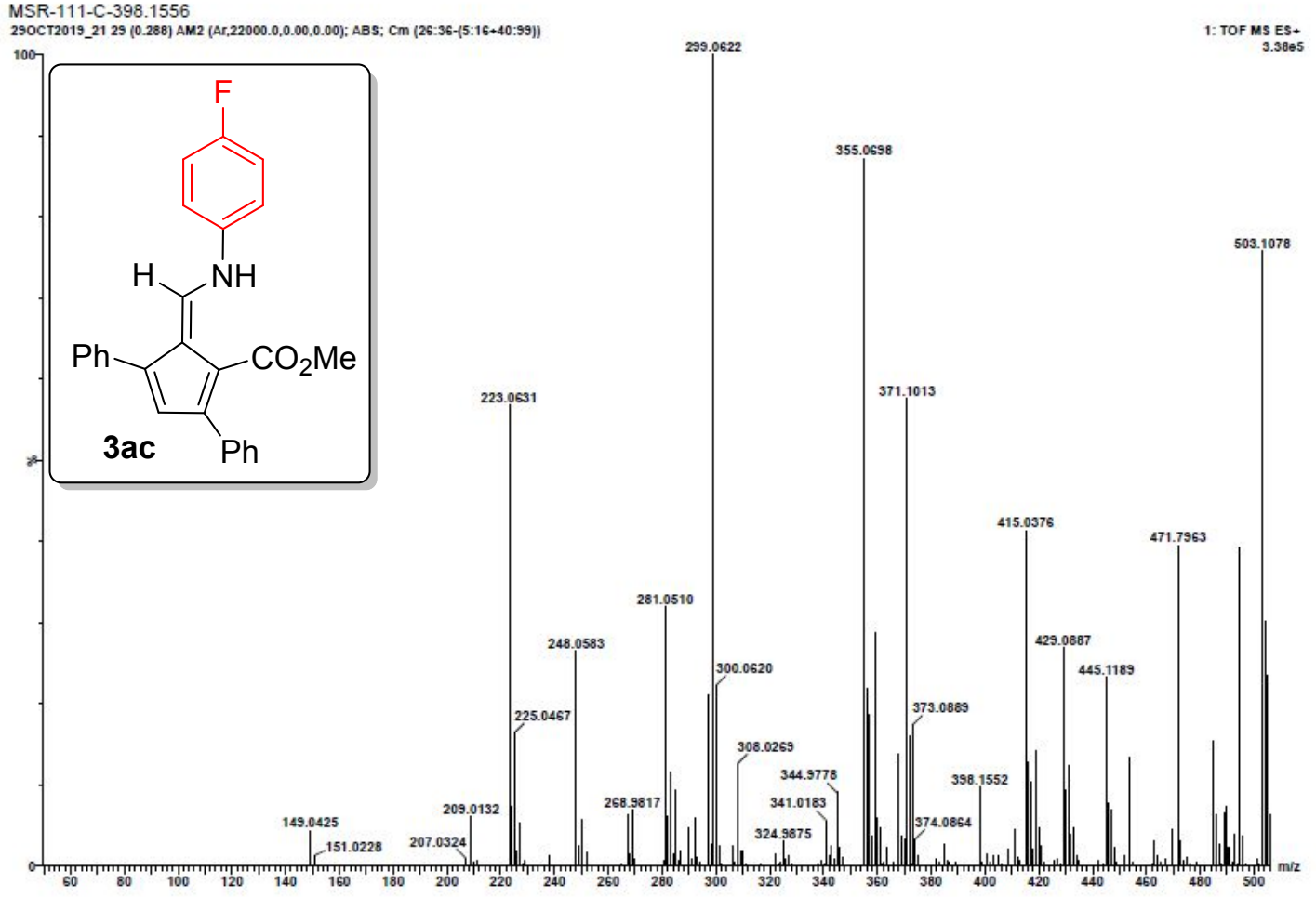



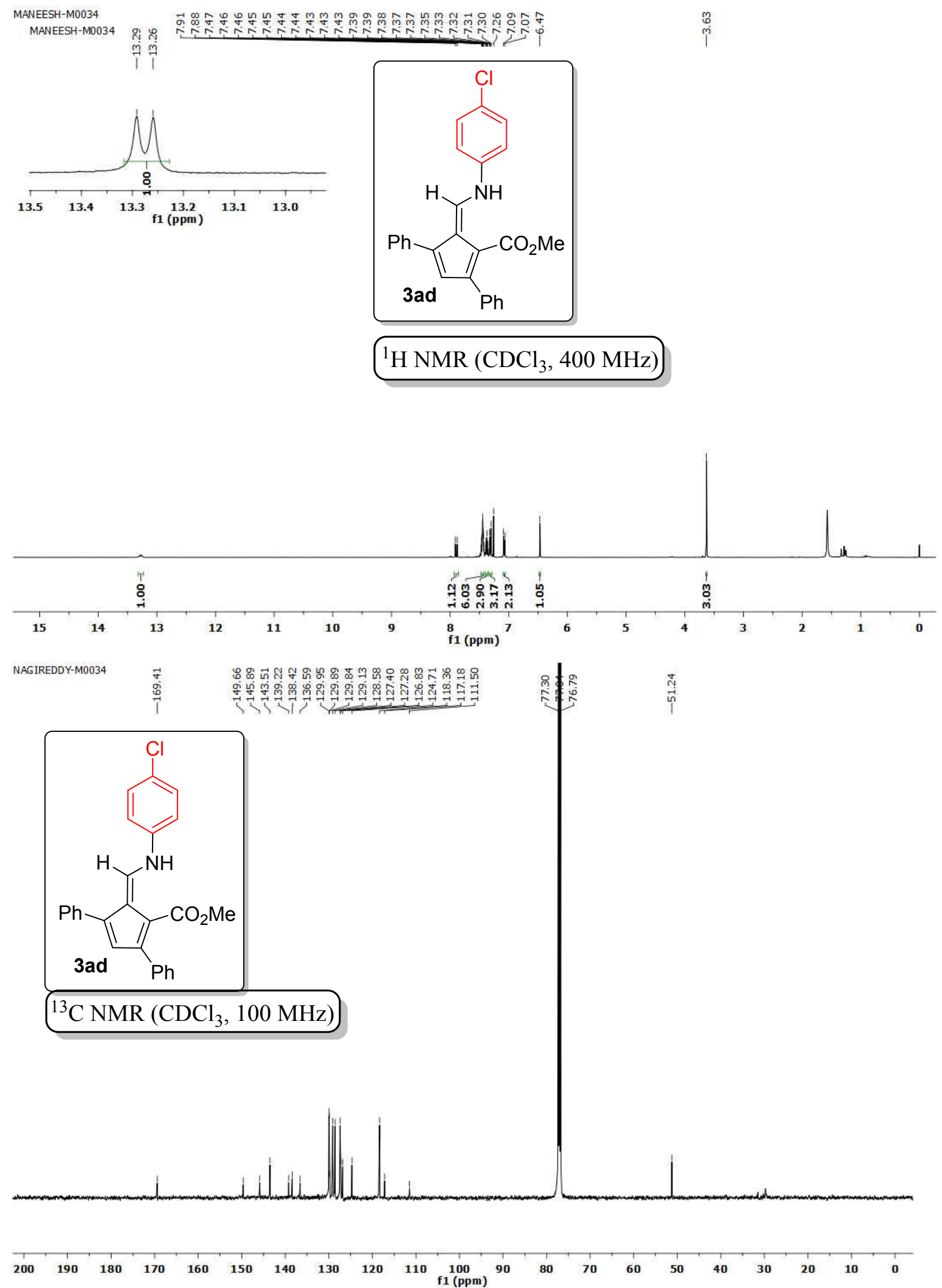
Maddi SRIDHAR REDDY

11/27/2019 3:45:27 PM

ThermoScientific EXACTIVE ORBITRAP

Analysed By G Saikrishna

MSR-64-C \#2-13 ${ }^{-} \mathrm{RT}^{-} \mathrm{C}^{-} 0.01-0.05^{-} \mathrm{AV}:{ }^{-} 12^{-} \mathrm{NL:}:{ }^{-1} .92 \mathrm{E} 8$

T: FTMS $\{1,1\}+p$ APCl corona Full ms [100.00-2000.00] 414.12589

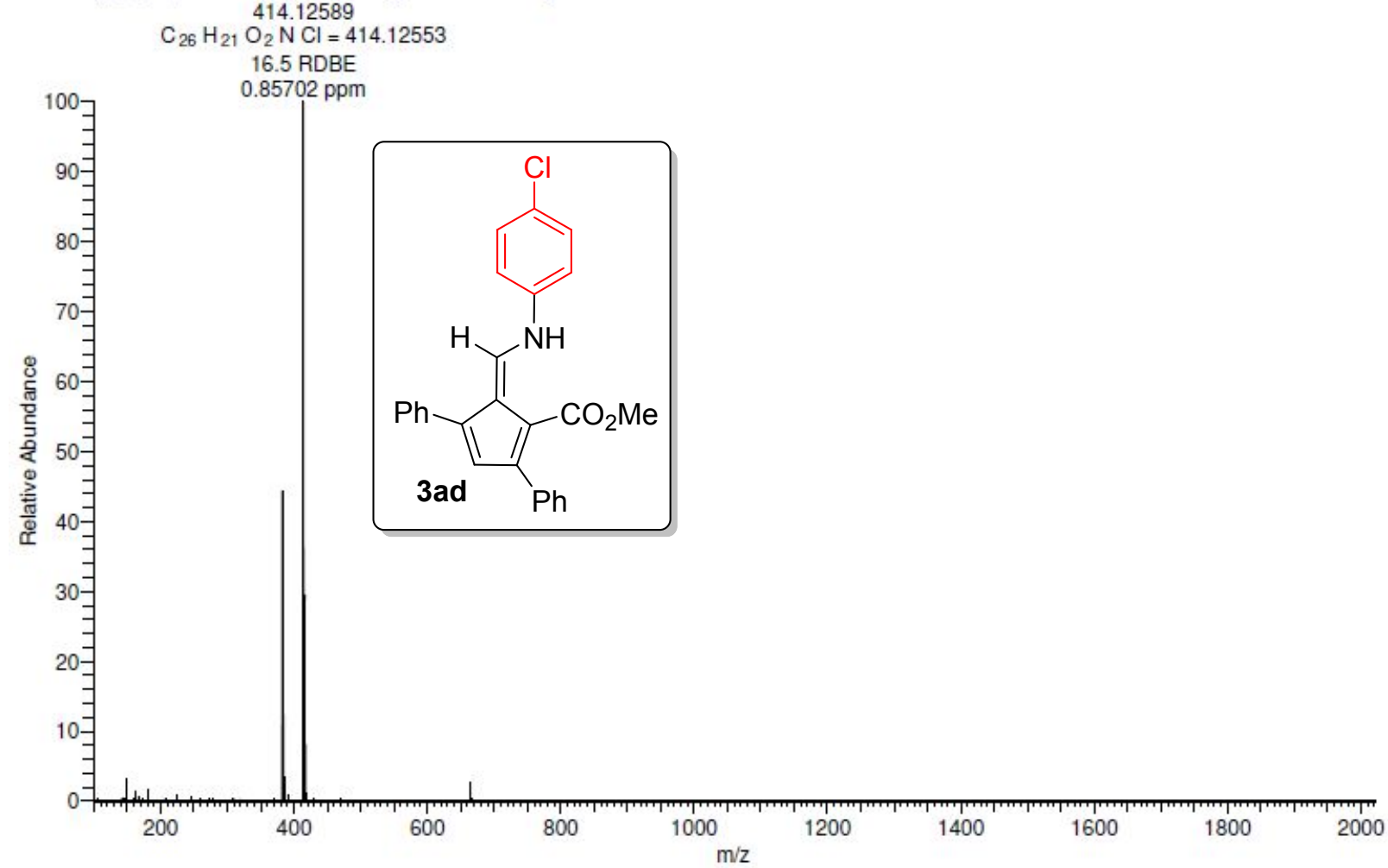

MANEESH-M0034

MANEESH-M0034

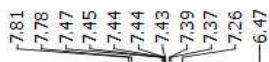

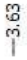
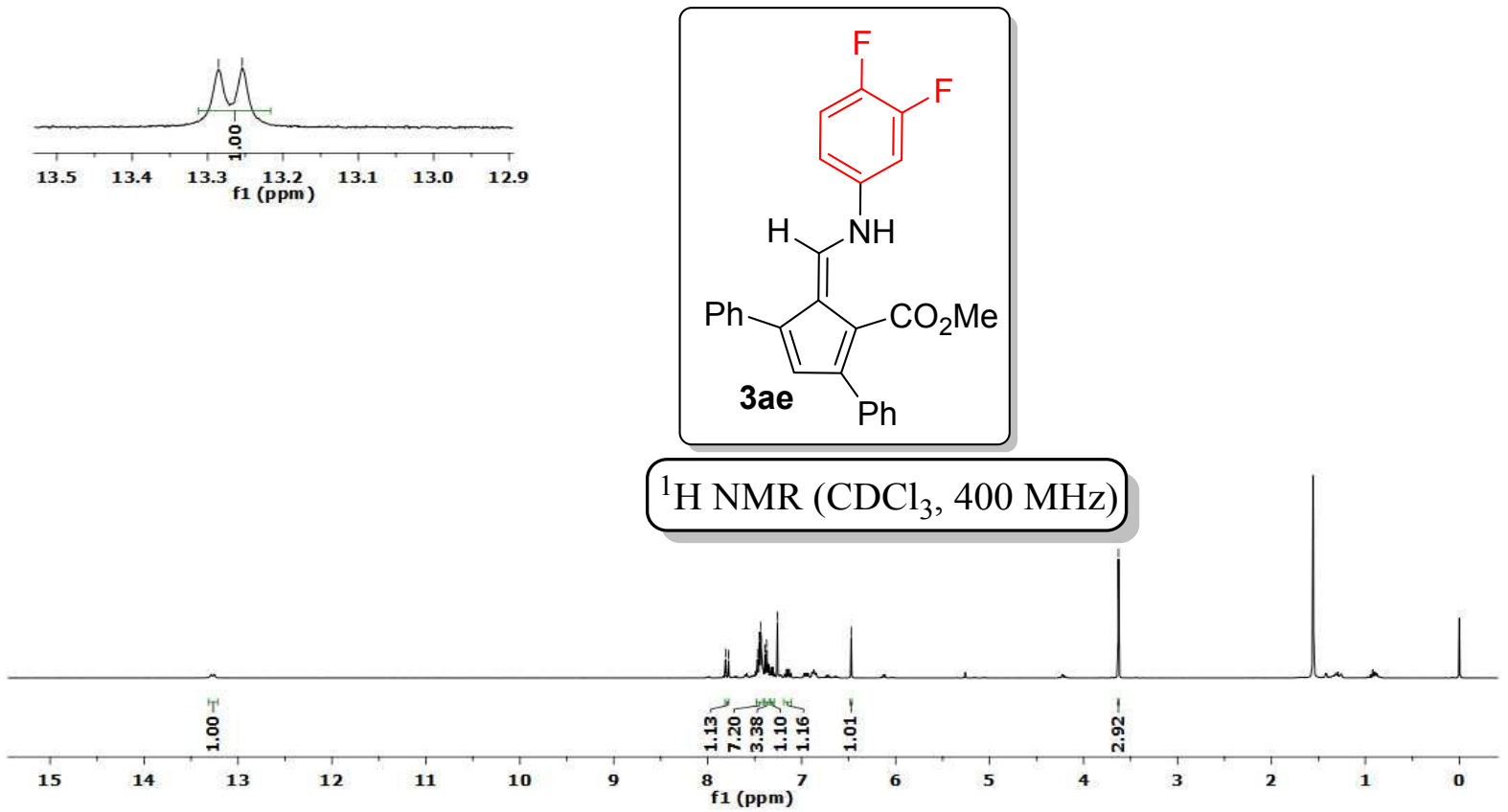

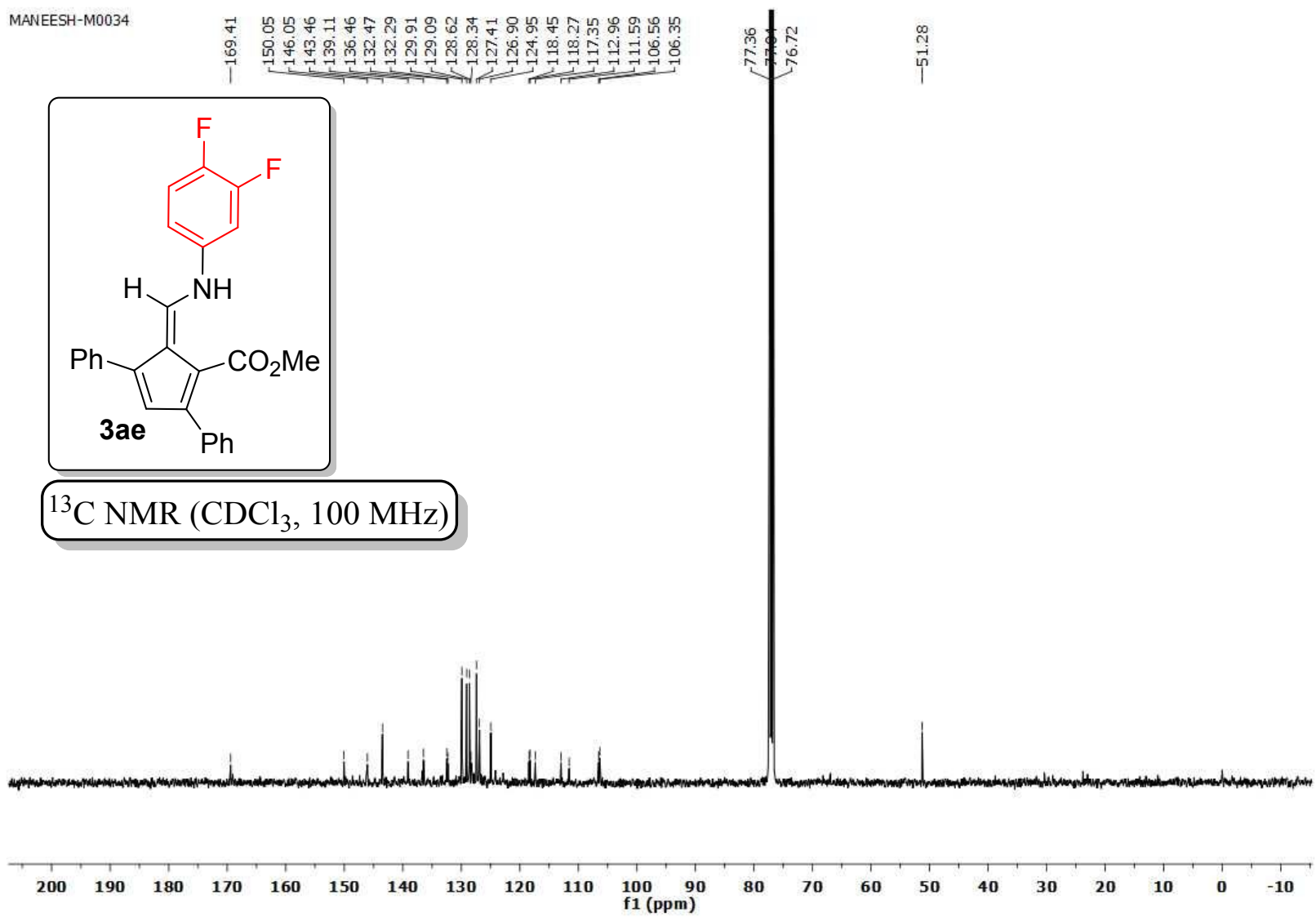

DATTA-H0011

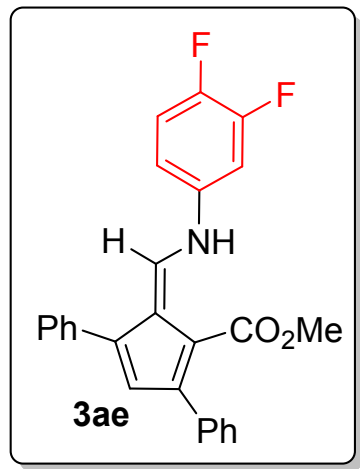

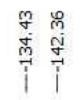

${ }^{19} \mathrm{~F} \mathrm{NMR}\left(\mathrm{CDCl}_{3}, 377 \mathrm{MHz}\right)$

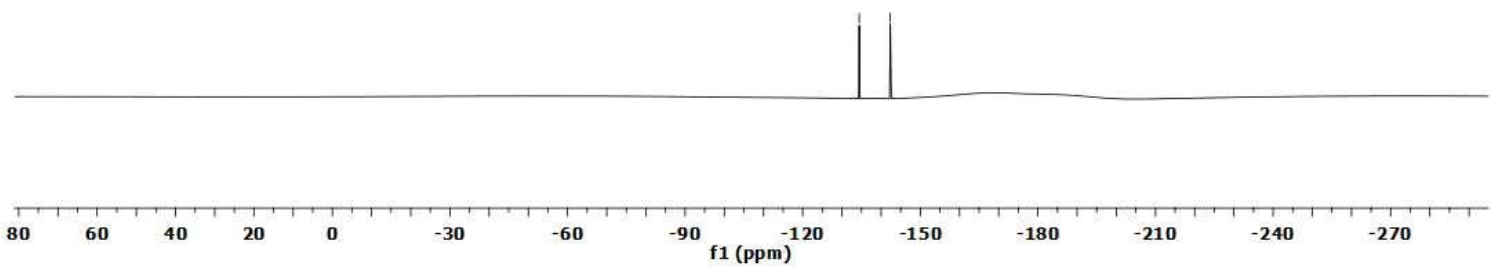


Maddi SRIDHAR REDDY

11/27/2019 4:06:02 PM

ThermoScientific EXACTIVE ORBITRAP

Analysed By G Saikrishna

MSR-115-C \#2-13- $\mathrm{RT}^{-}{ }^{-} 0.01-0.05^{-} \mathrm{AV}^{-}{ }^{-} 12^{-} \mathrm{NL}^{-}{ }^{-} 2.57 \mathrm{E} 8$

T: FTMS $\{1,1\}+$ p APCl corona Full ms [100.00-2000.00]

416.14559

$\mathrm{C}_{26} \mathrm{H}_{20} \mathrm{O}_{2} \mathrm{~N} \mathrm{~F}_{2}=416.14566$

16.5 RDBE

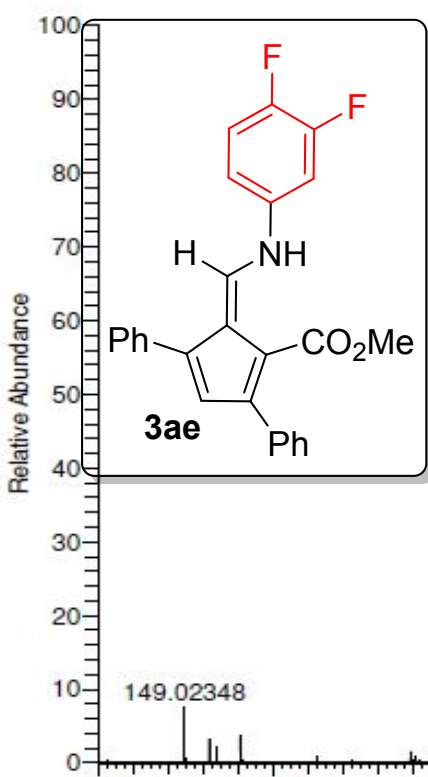

$-0.17746 \mathrm{ppm}$

100

200

300

400

500

$m / z$ 

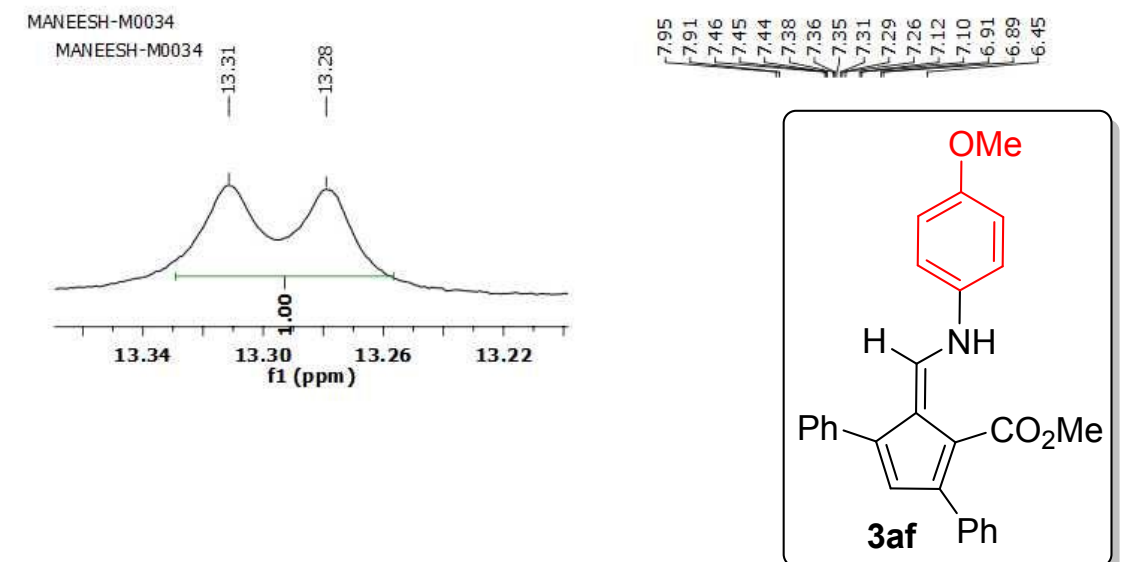

${ }^{1} \mathrm{H} \mathrm{NMR}\left(\mathrm{CDCl}_{3}, 400 \mathrm{MHz}\right)$

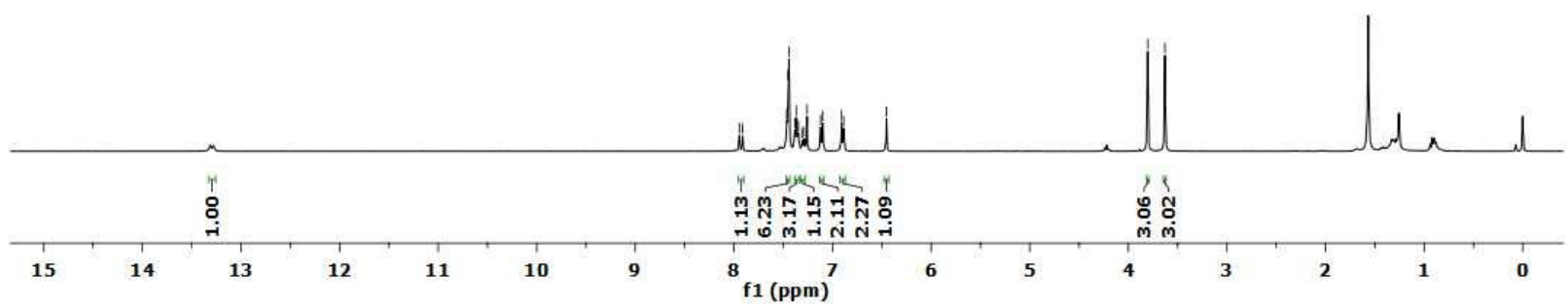

${ }^{13} \mathrm{C} \mathrm{NMR}\left(\mathrm{CDCl}_{3}, 100 \mathrm{MHz}\right)$
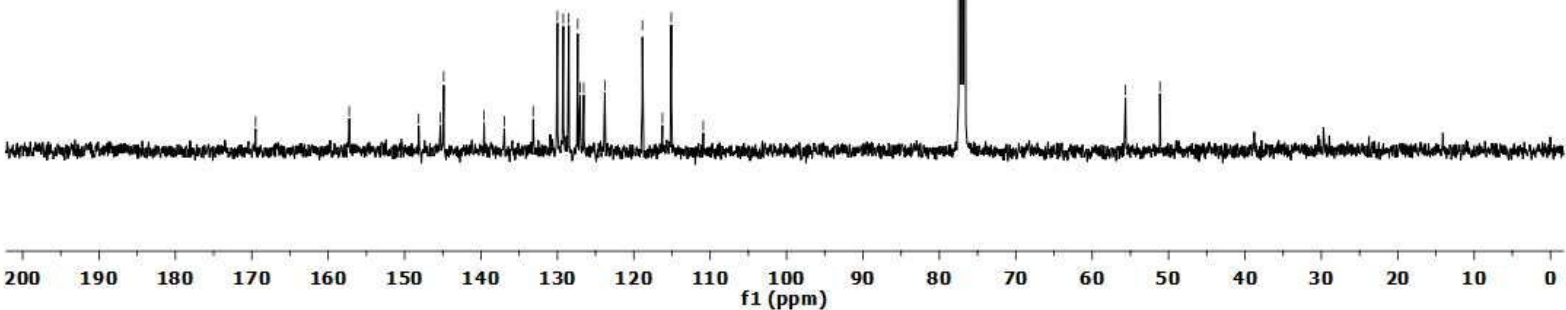


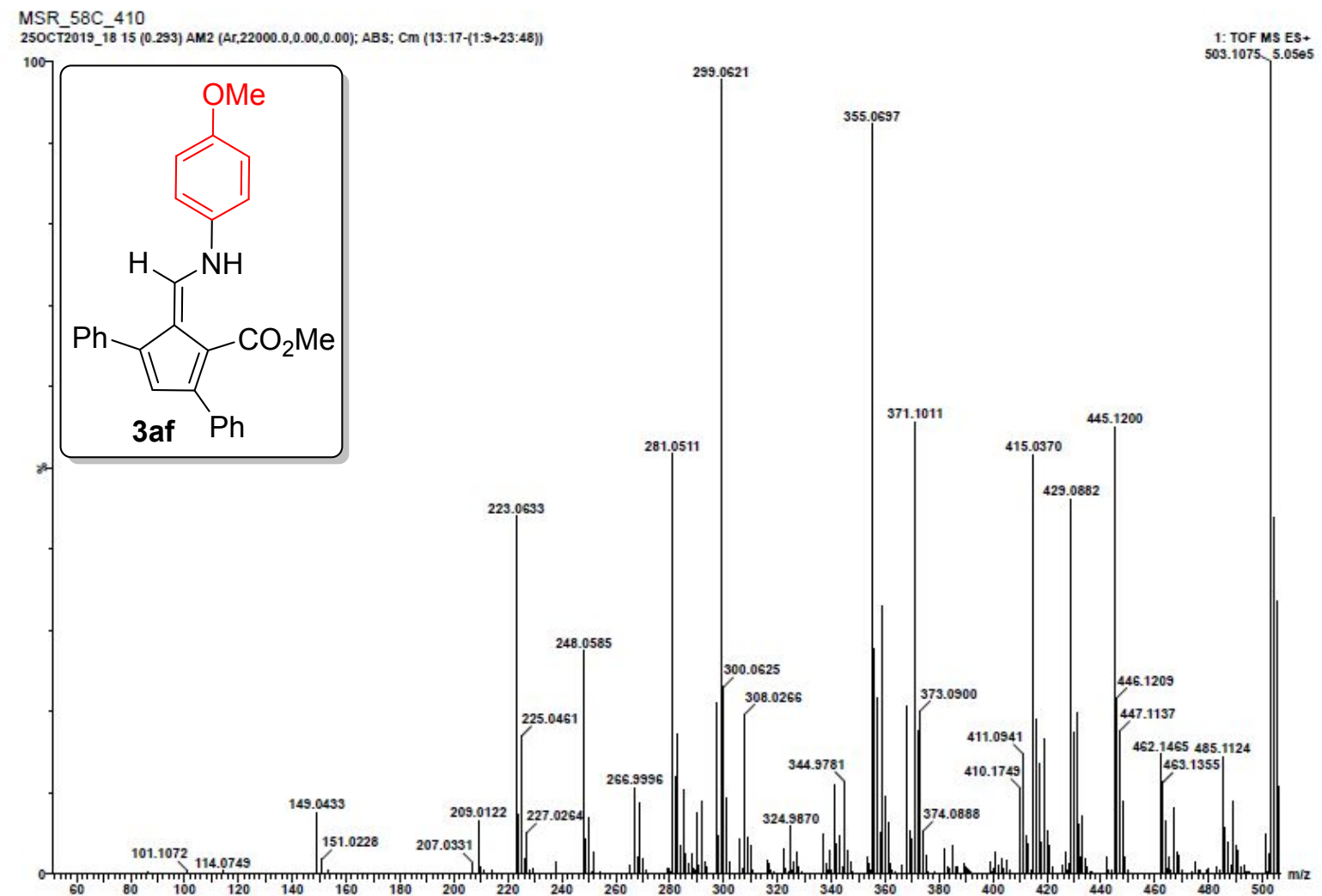



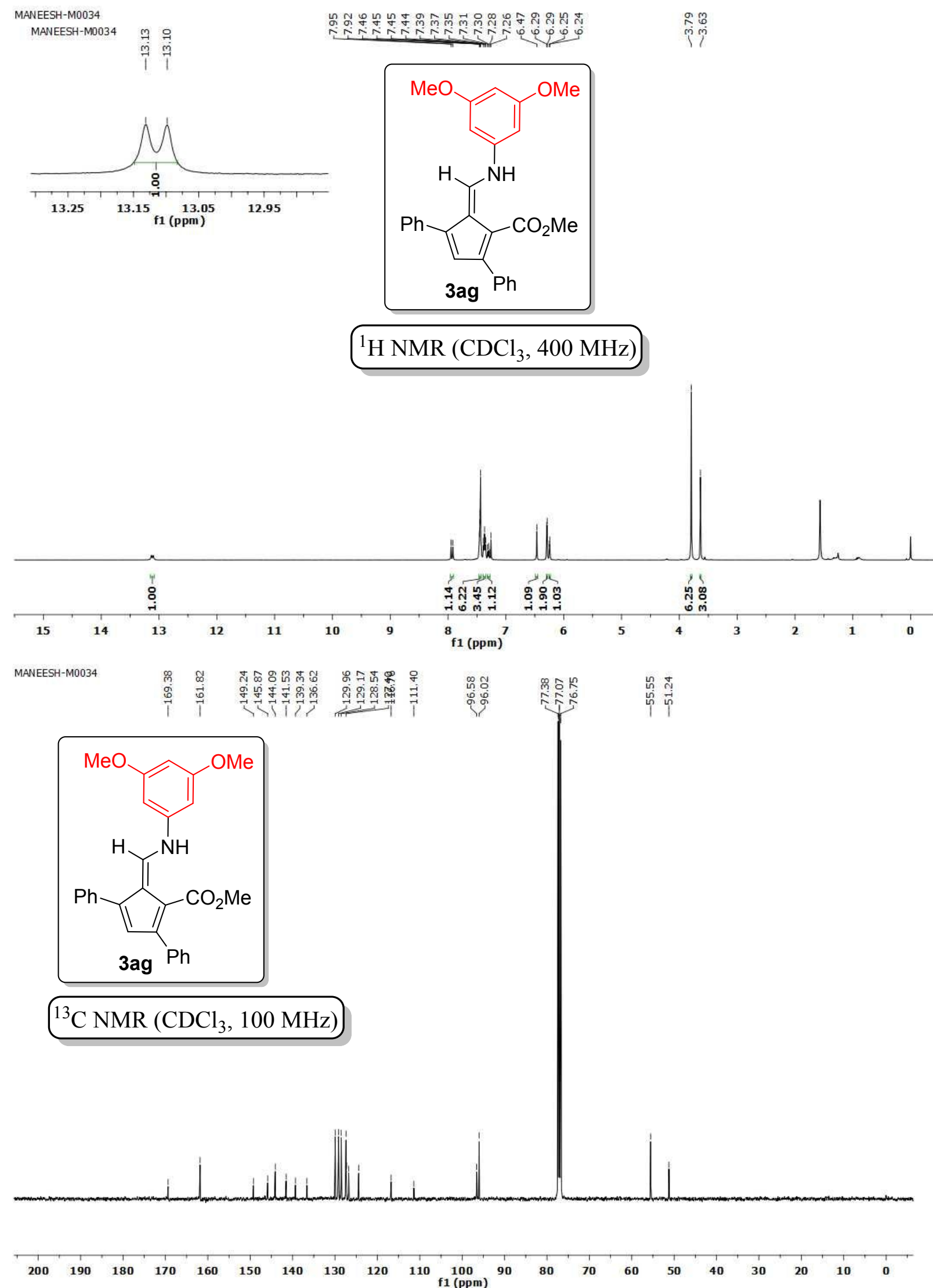
Maddi SRIDHAR REDDY

11/27/2019 4:11:10 PM

ThermoScientific EXACTIVE ORBITRAP

Analysed By G Saikrishna

MSR-58-D \#2-13- ${ }^{-} T^{-}{ }^{-} 0.01-0.05^{-}$AV: $^{-} 12^{-} \mathrm{NL}:-6.40 \mathrm{E}$

T: FTMS $\{1,1\}+p$ APCl corona Full ms [100.00-2000.00] 440.18604 $\mathrm{C}_{28} \mathrm{H}_{26} \mathrm{O}_{4} \mathrm{~N}=440.18563$

16.5 RDBE

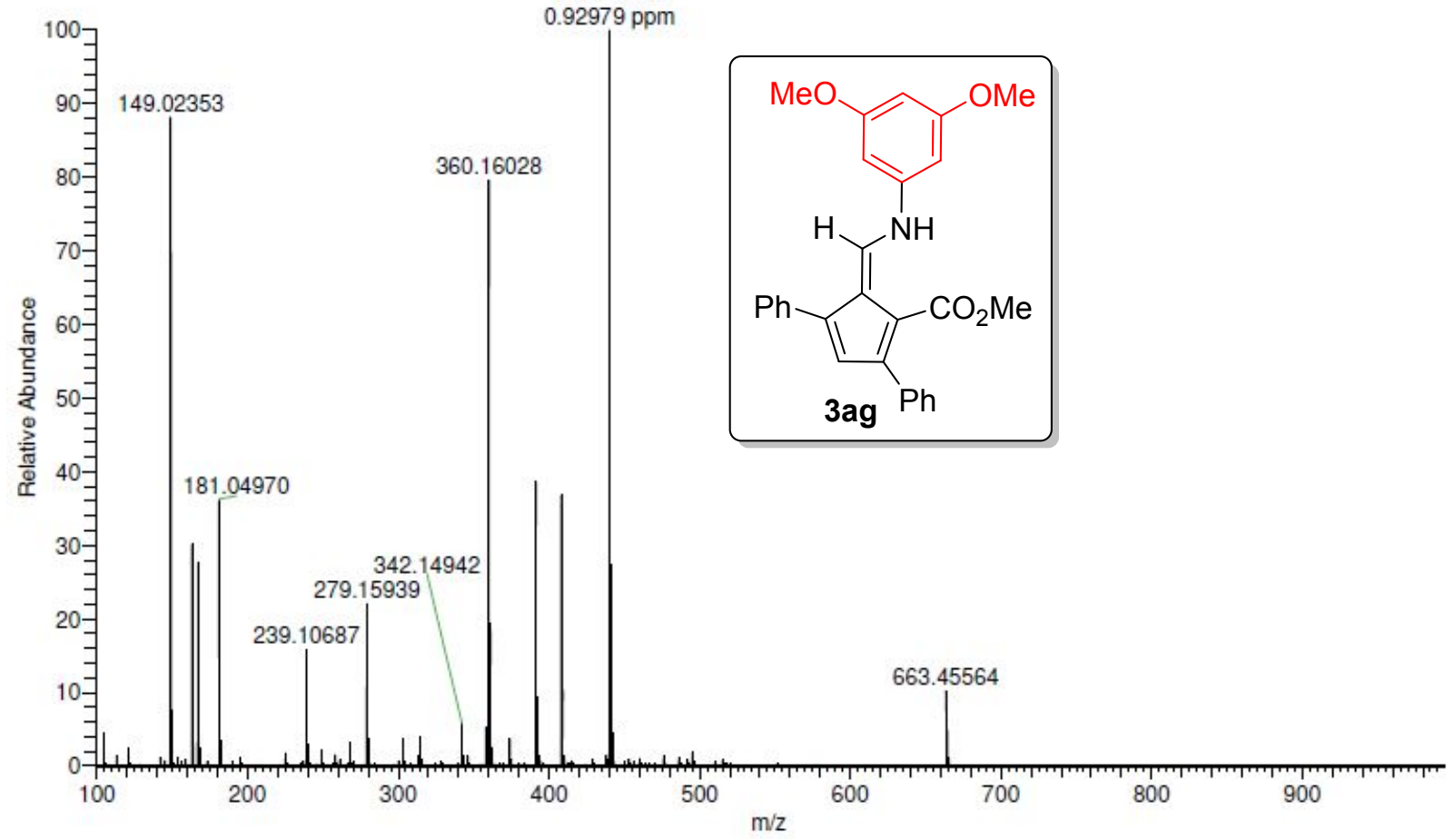

BAGI-M0034

BAGI-M0034
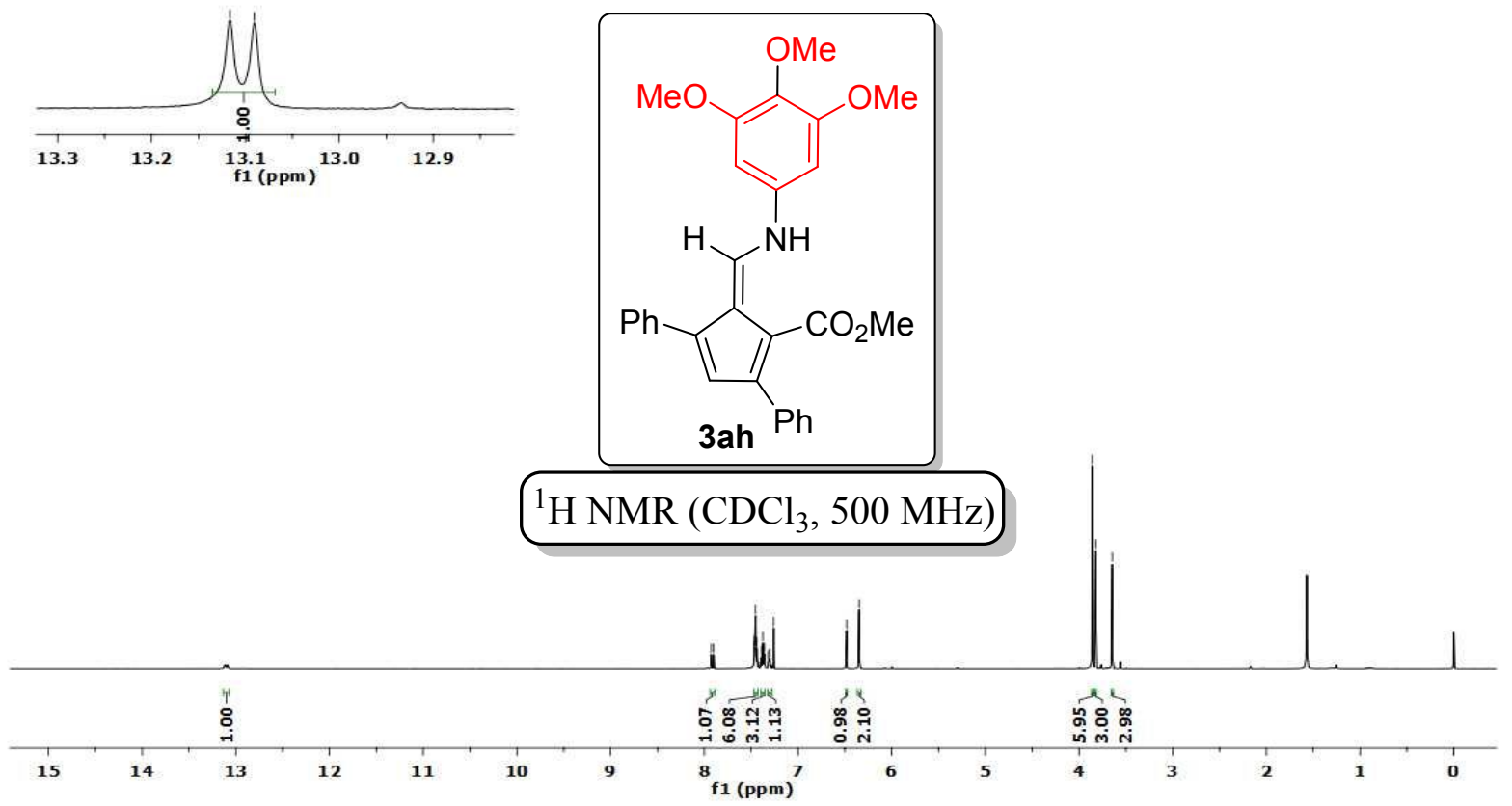

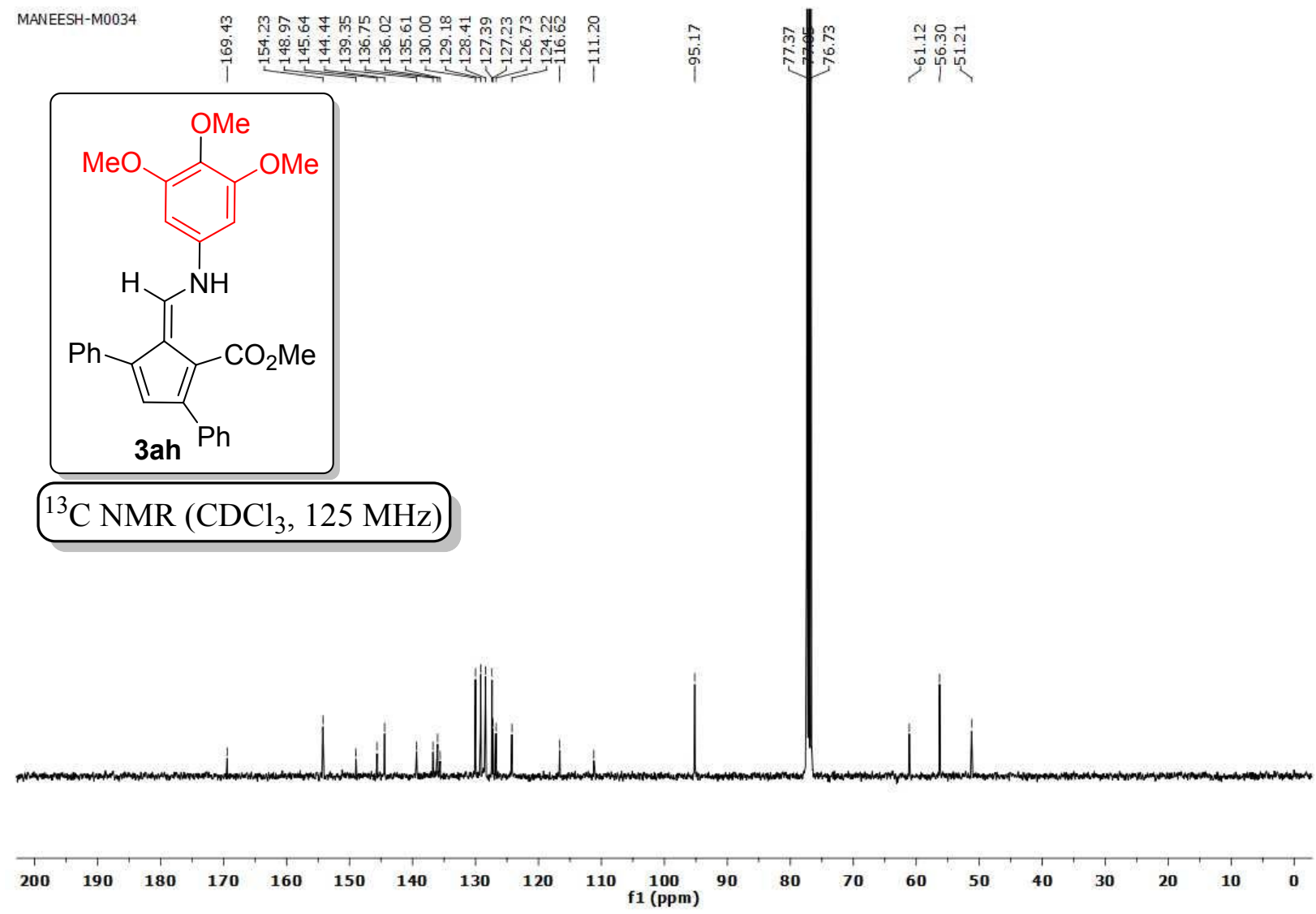

Maddi SRIDHAR REDDY

11/27/2019 3.48:02 PM

ORBITRAP

y G Saikrishna

MSR-118-C \#2-13 ${ }^{-} \mathrm{RT}^{-}{ }^{-} 0.01-0.05^{-} \mathrm{AV}^{-} 12^{-} \mathrm{NL}:{ }^{-} 2.87 \mathrm{E} 8$

T: FTMS $\{1,1\}+\mathrm{p} \mathrm{APCl}$ corona Full ms [100.00-2000.00] 470.19576

$\mathrm{C}_{29} \mathrm{H}_{28} \mathrm{O}_{5} \mathrm{~N}=470.19620$

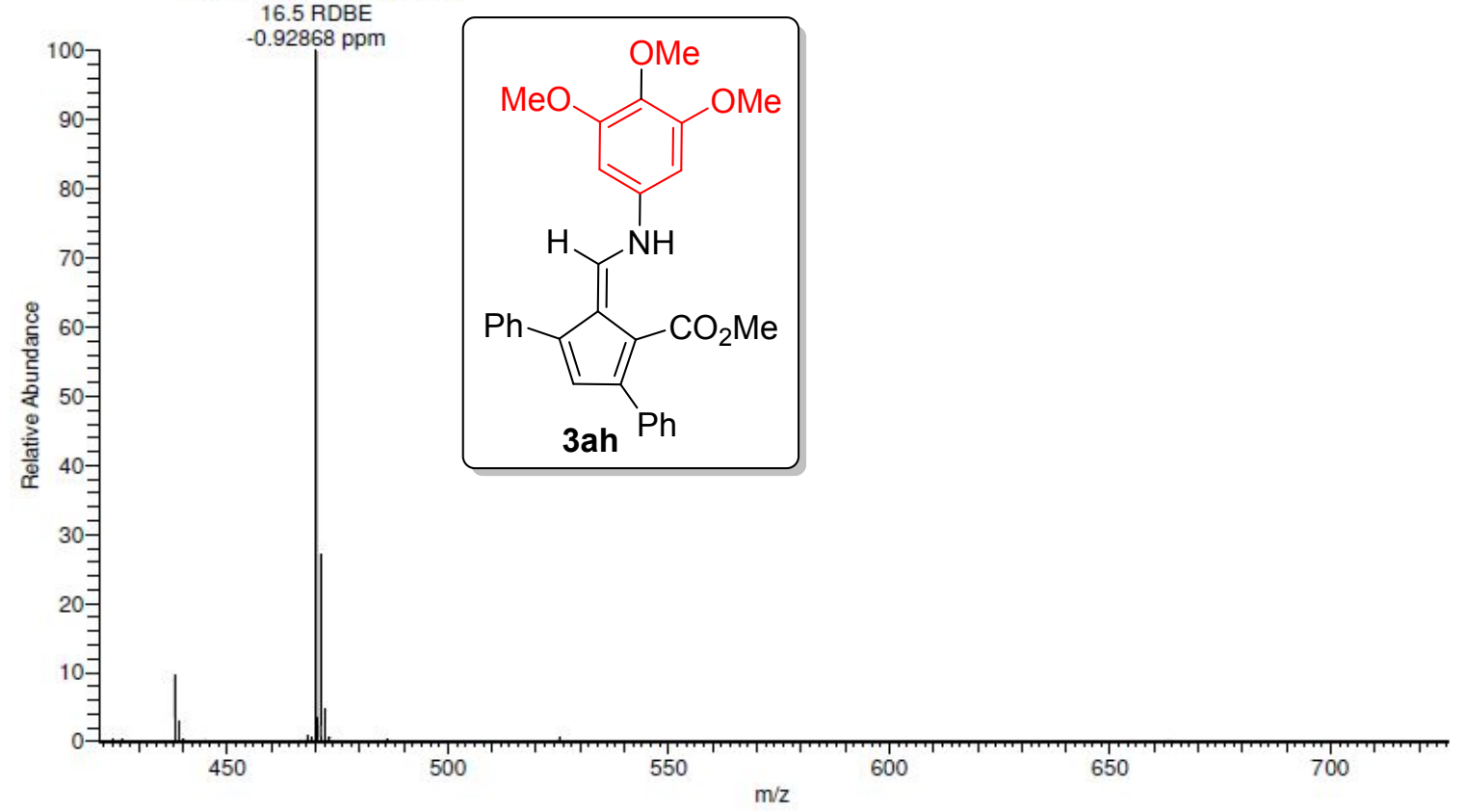



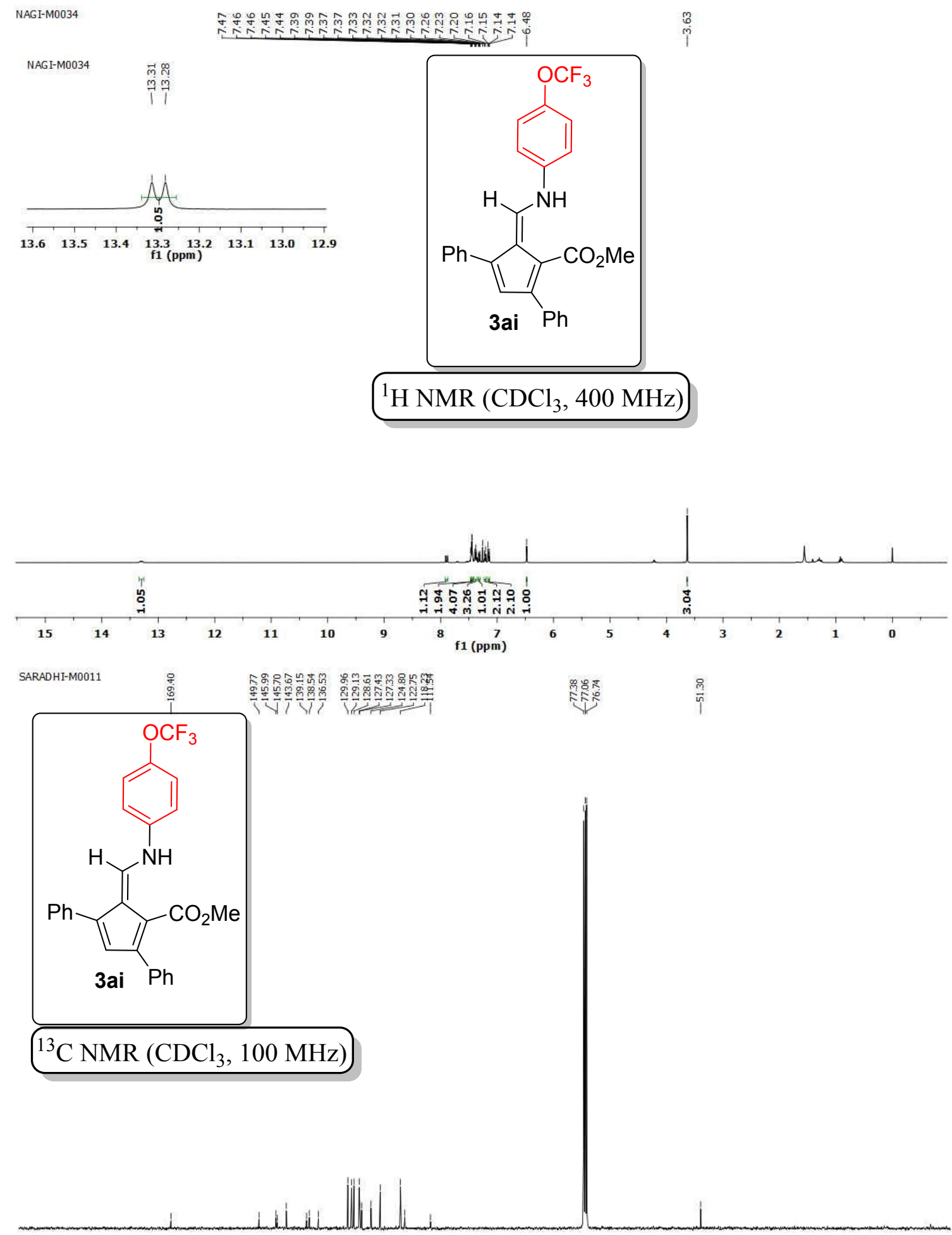


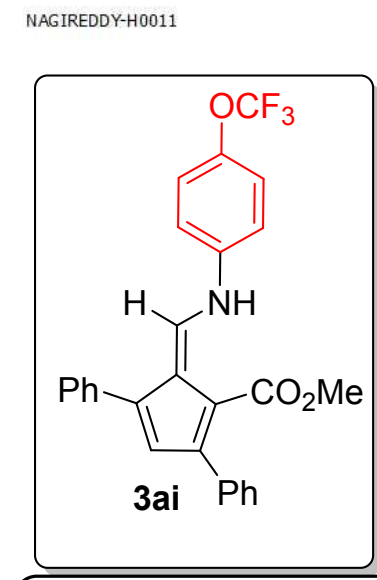

${ }^{19} \mathrm{~F} \mathrm{NMR}\left(\mathrm{CDCl}_{3}, 377 \mathrm{MHz}\right)$

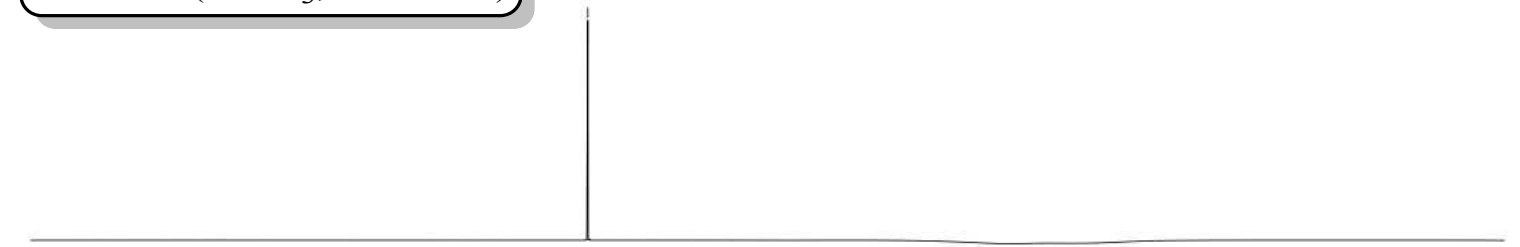

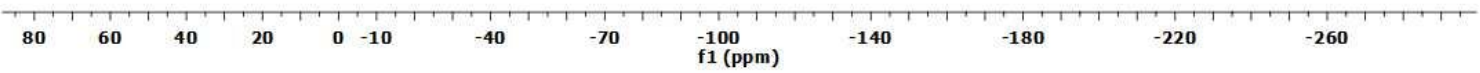

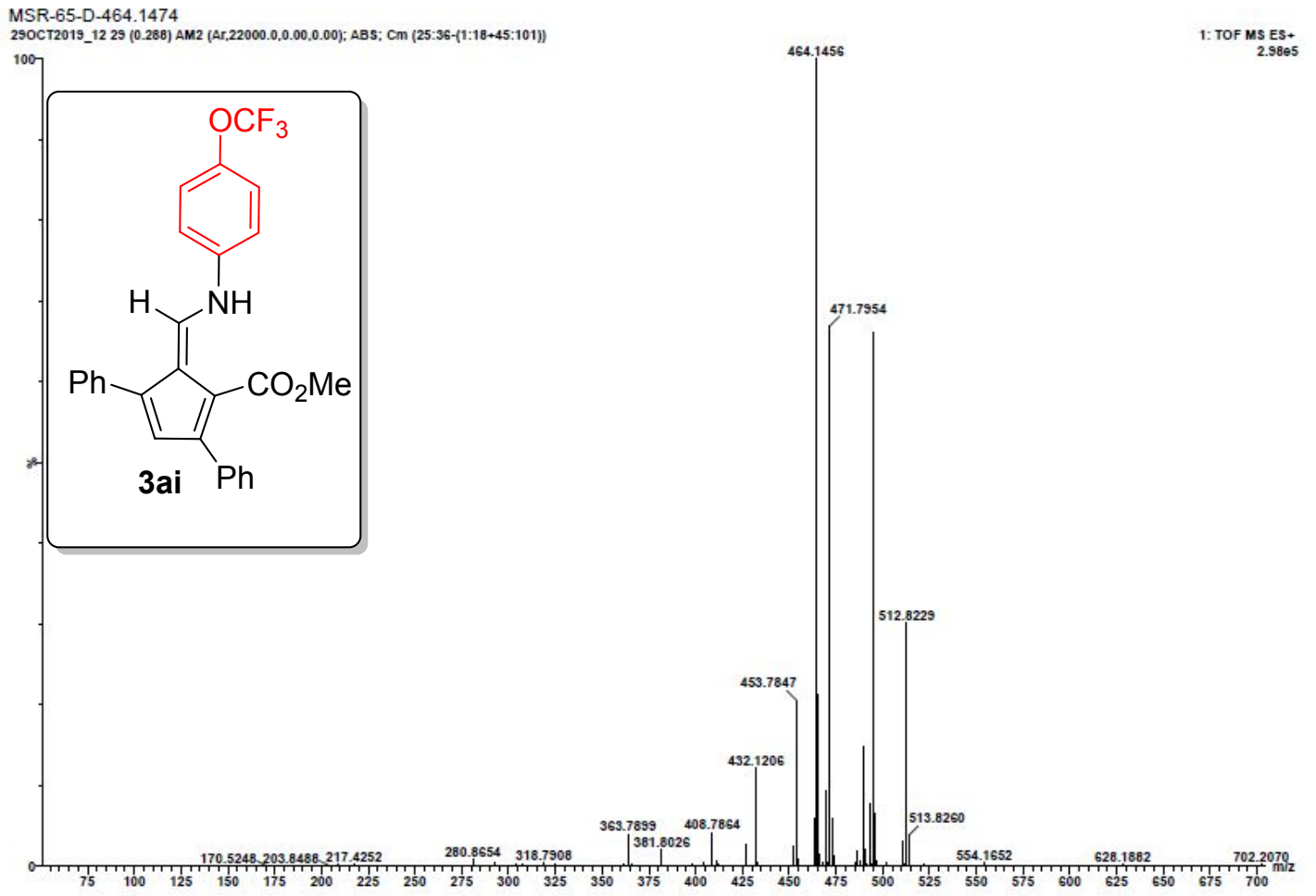



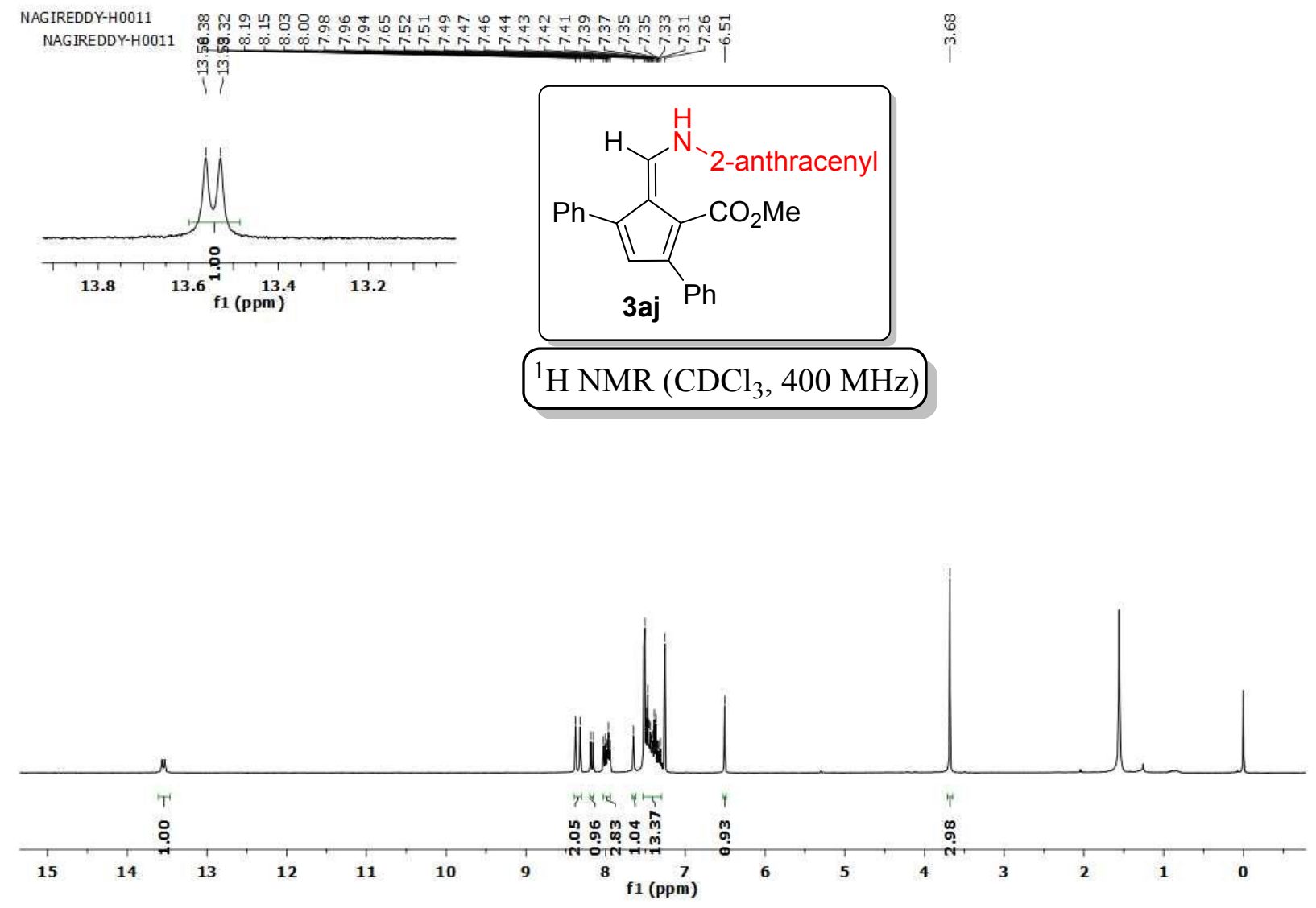

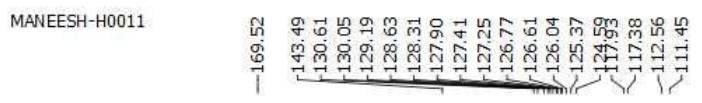

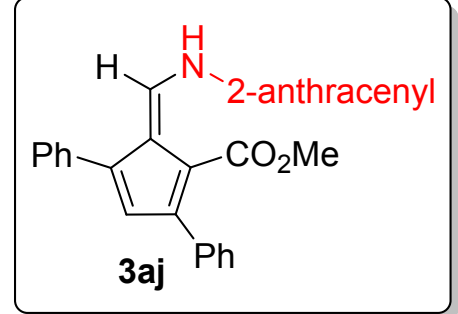

${ }^{13} \mathrm{C} \mathrm{NMR}\left(\mathrm{CDCl}_{3}, 100 \mathrm{MHz}\right)$

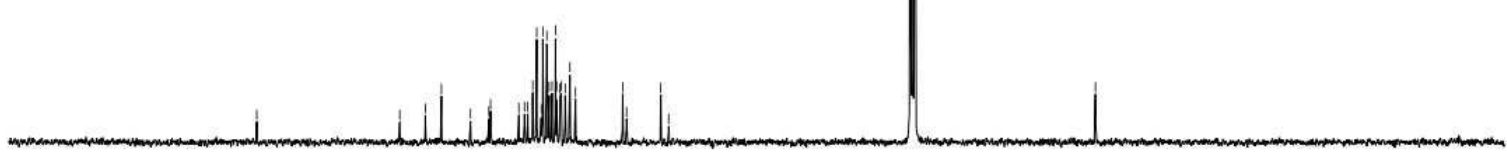

$\begin{array}{llllllllllllllllllllll}200 & 190 & 180 & 170 & 160 & 150 & 140 & 130 & 120 & 110 & 100 & 90 & 80 & 70 & 60 & 50 & 40 & 30 & 20 & 10 & 0\end{array}$ 
Maddi SRIDHAR REDDY

11/27/2019 4:13:43 PM

ThermoScientific EXACTIVE ORBITRAP

Analysed By G Saikrishna

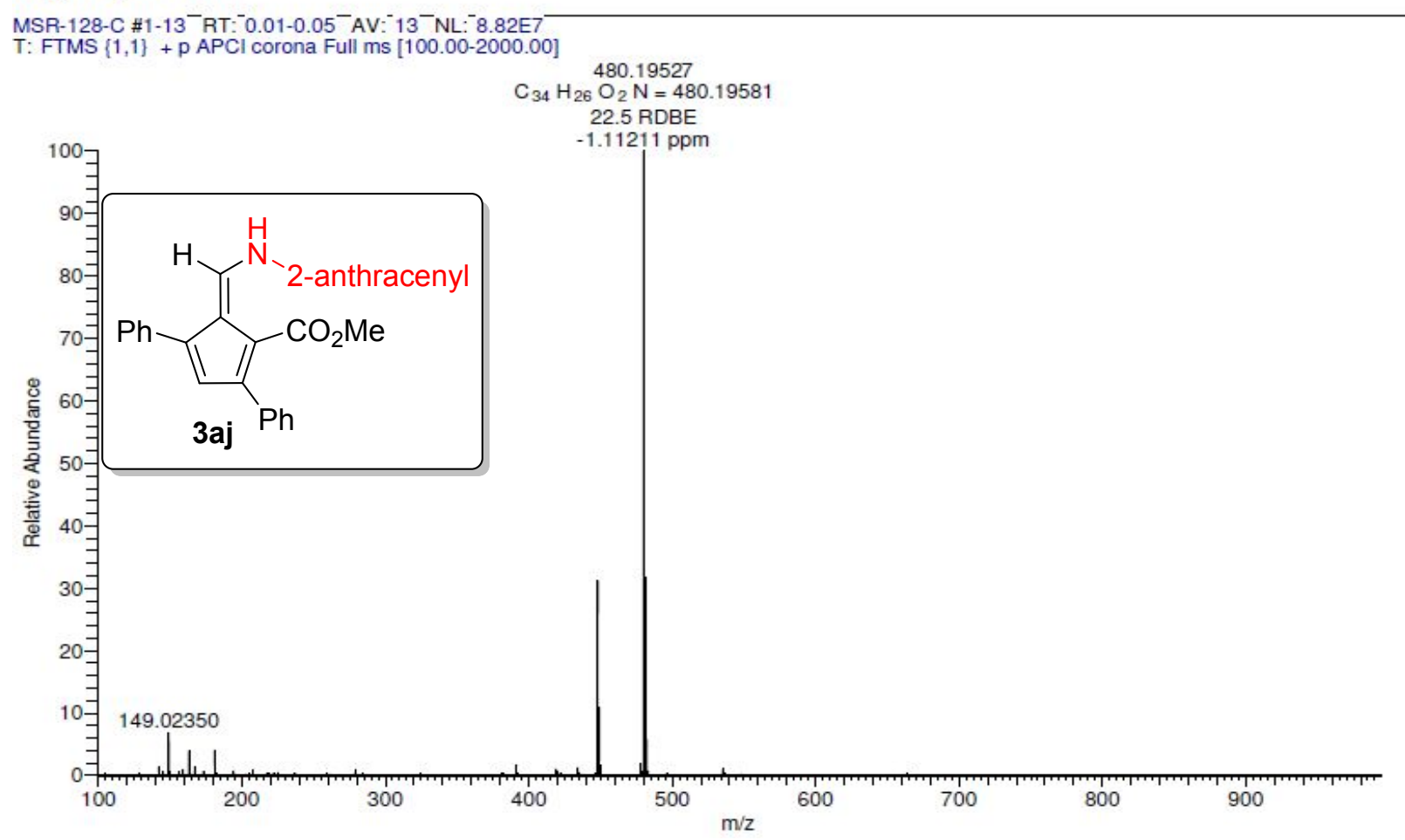

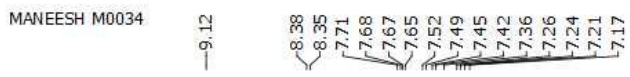

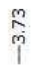

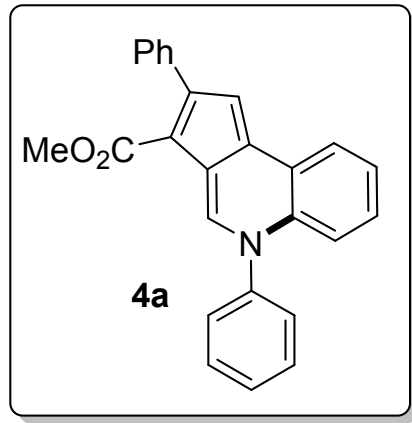

${ }^{1} \mathrm{H} \mathrm{NMR}\left(\mathrm{CDCl}_{3}, 400 \mathrm{MHz}\right)$

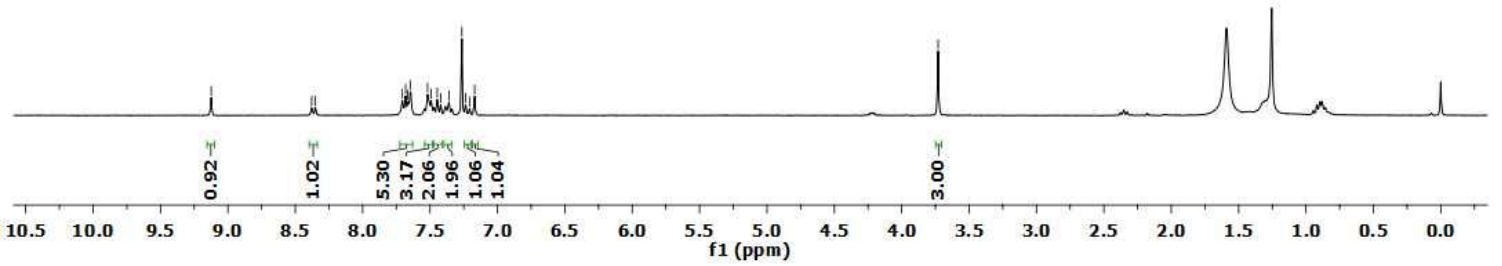


MANEESH-M0034

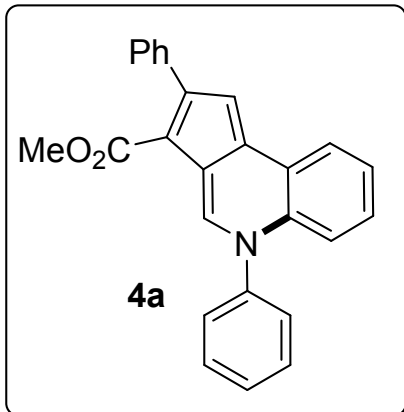

${ }^{13} \mathrm{C} \mathrm{NMR}\left(\mathrm{CDCl}_{3}, 100 \mathrm{MHz}\right)$
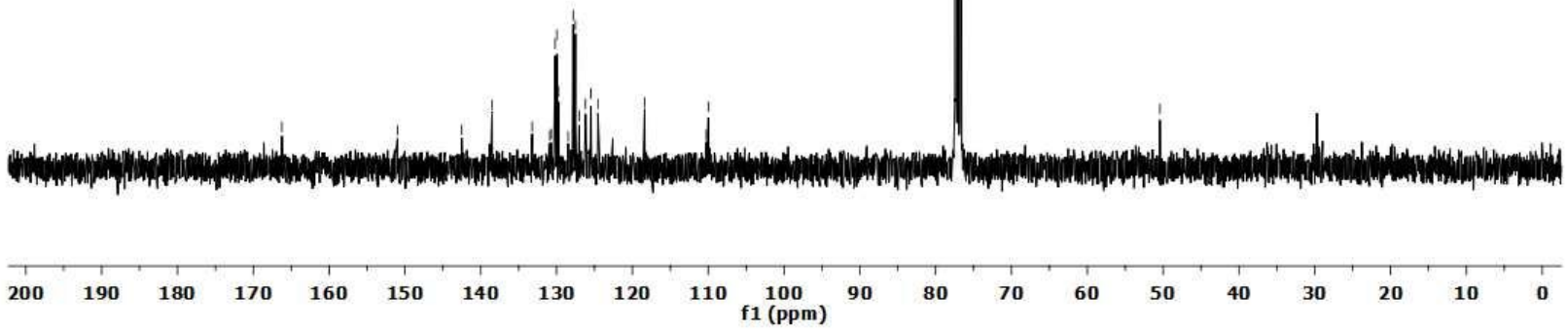

Maddi SRIDHAR REDDY

11/27/2019 3:58:19 PM

ThermoScientific EXACTIVE ORBITRAP

Analysed By G Saikrishna

MSR-69-B \#2-13- ${ }^{-} T^{-}{ }^{-} 0.01-0.05^{-} \mathrm{AV}^{-}{ }^{-1}{ }^{-} \mathrm{NL}:{ }^{-} 1.87 \mathrm{E8}$

T: FTMS $\{1,1\}+$ p APCl corona Full ms [100.00-2000.00]

378.14962
$\mathrm{C}_{26} \mathrm{H}_{20} \mathrm{O}_{2} \mathrm{~N}=378.14886$

$2.01540 \mathrm{ppm}$

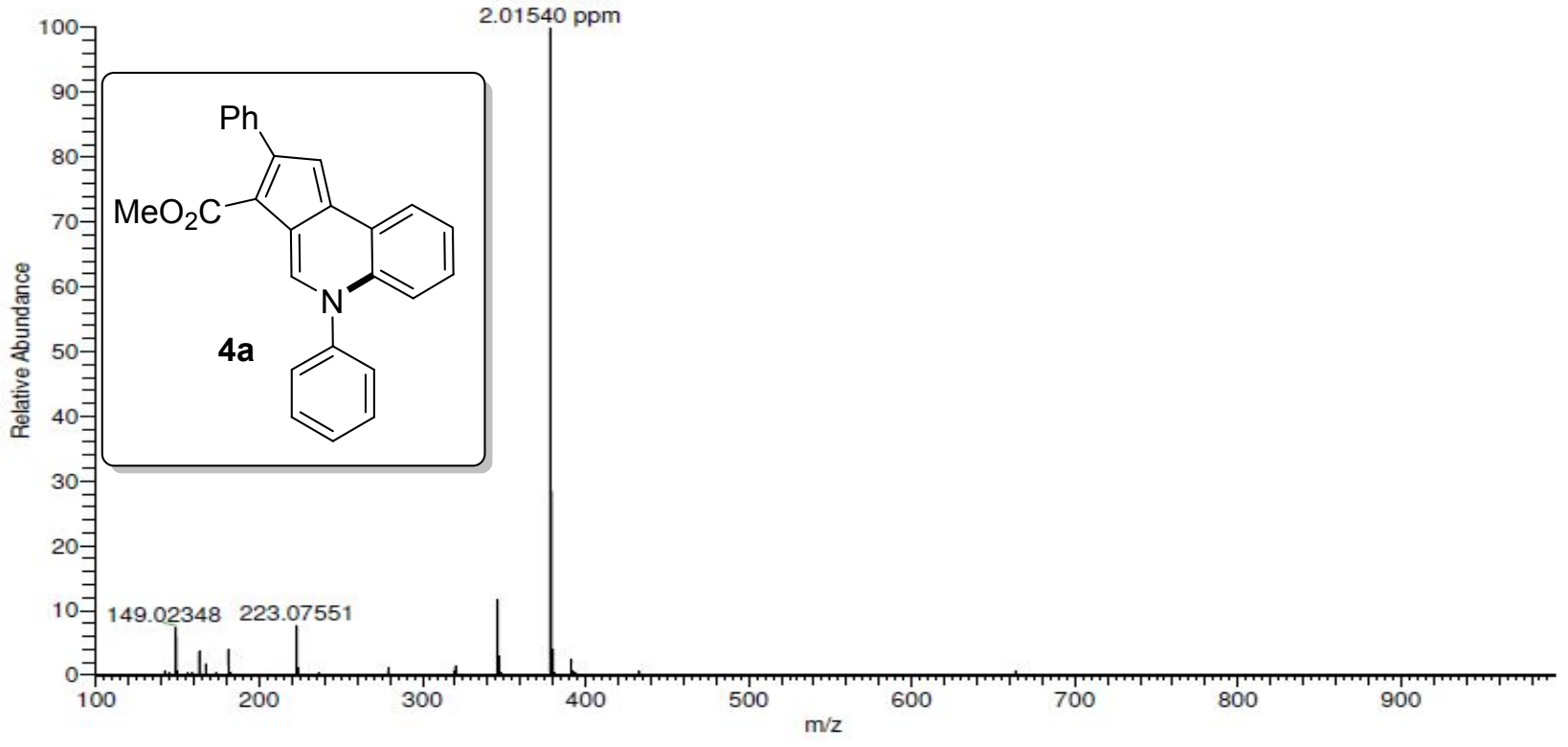




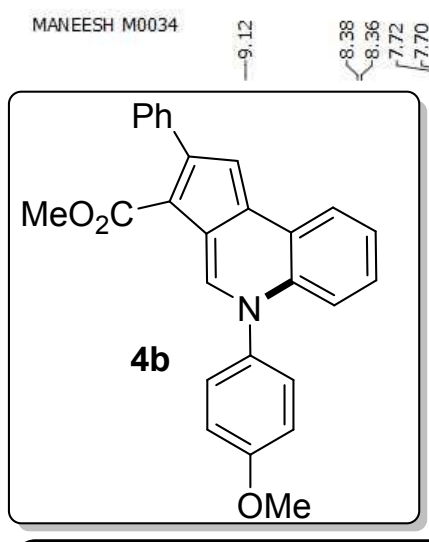

${ }^{1} \mathrm{H} \mathrm{NMR}\left(\mathrm{CDCl}_{3}, 300 \mathrm{MHz}\right)$

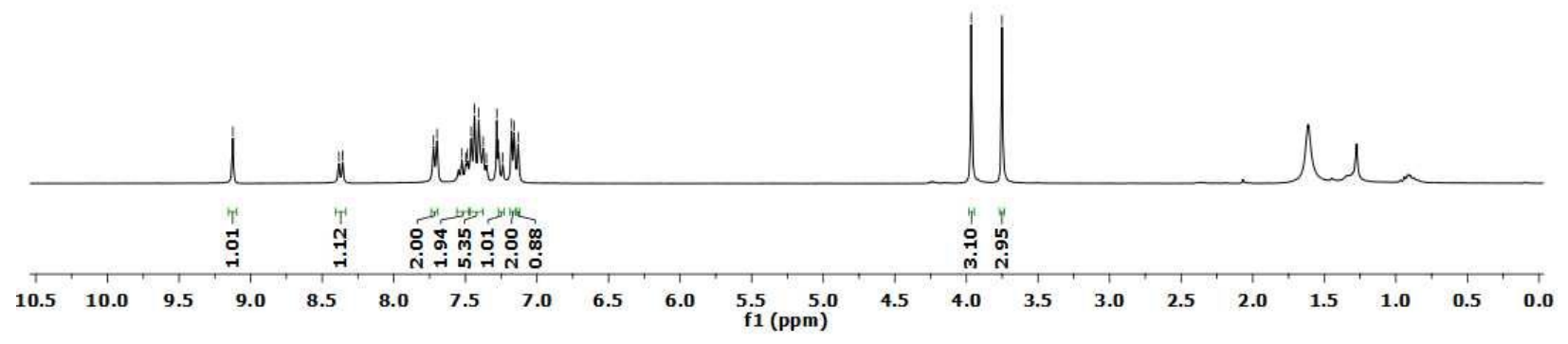

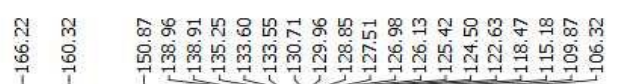

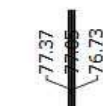

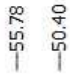

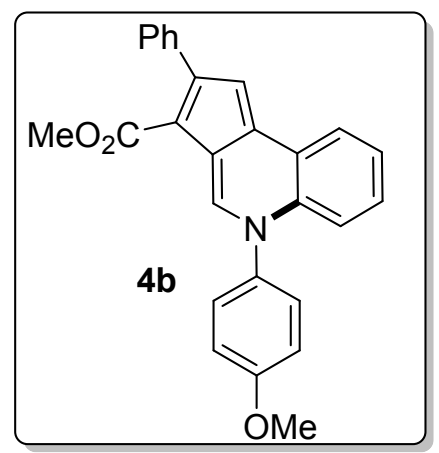

${ }^{13} \mathrm{C} \mathrm{NMR}\left(\mathrm{CDCl}_{3}, 75 \mathrm{MHz}\right)$
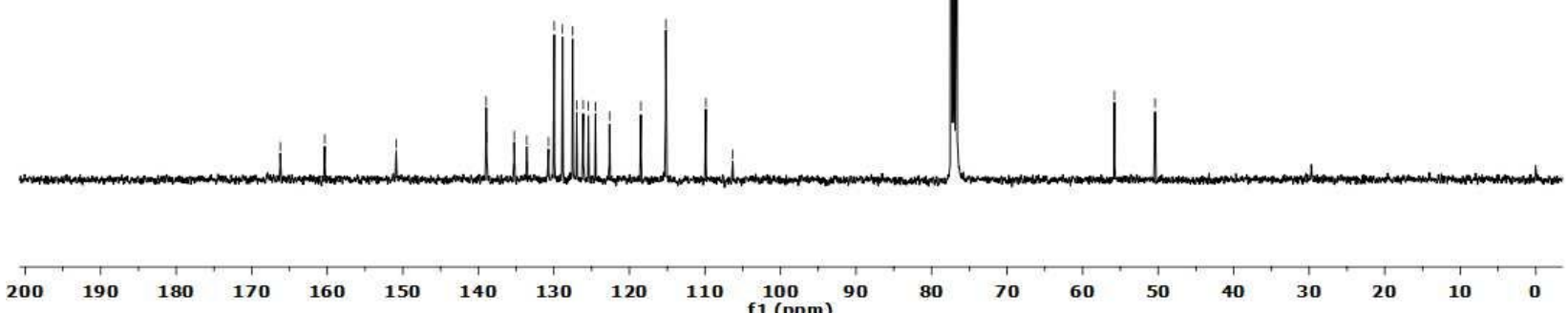
Maddi SRIDHAR REDDY

11/27/2019 4:08:35 PM

ThermoScientific EXACTIVE ORBITRAP

Analysed By G Saikrishna

MSR-143-A \#2-14 ${ }^{-} \mathrm{RT}^{-} \mathrm{-}^{-0} 0.01-0.06^{-} \mathrm{AV}^{-}{ }^{-13}{ }^{-} \mathrm{NL}:{ }^{-} 1.36 \mathrm{E} 8$

T: FTMS $\{1,1\}+$ p APCl corona Full ms [100.00-2000.00]

408.15965

$\mathrm{C}_{27} \mathrm{H}_{22} \mathrm{O}_{3} \mathrm{~N}=408.15942$

$17.5 \mathrm{RDBE}$
100
$10.57441 \mathrm{ppm}$

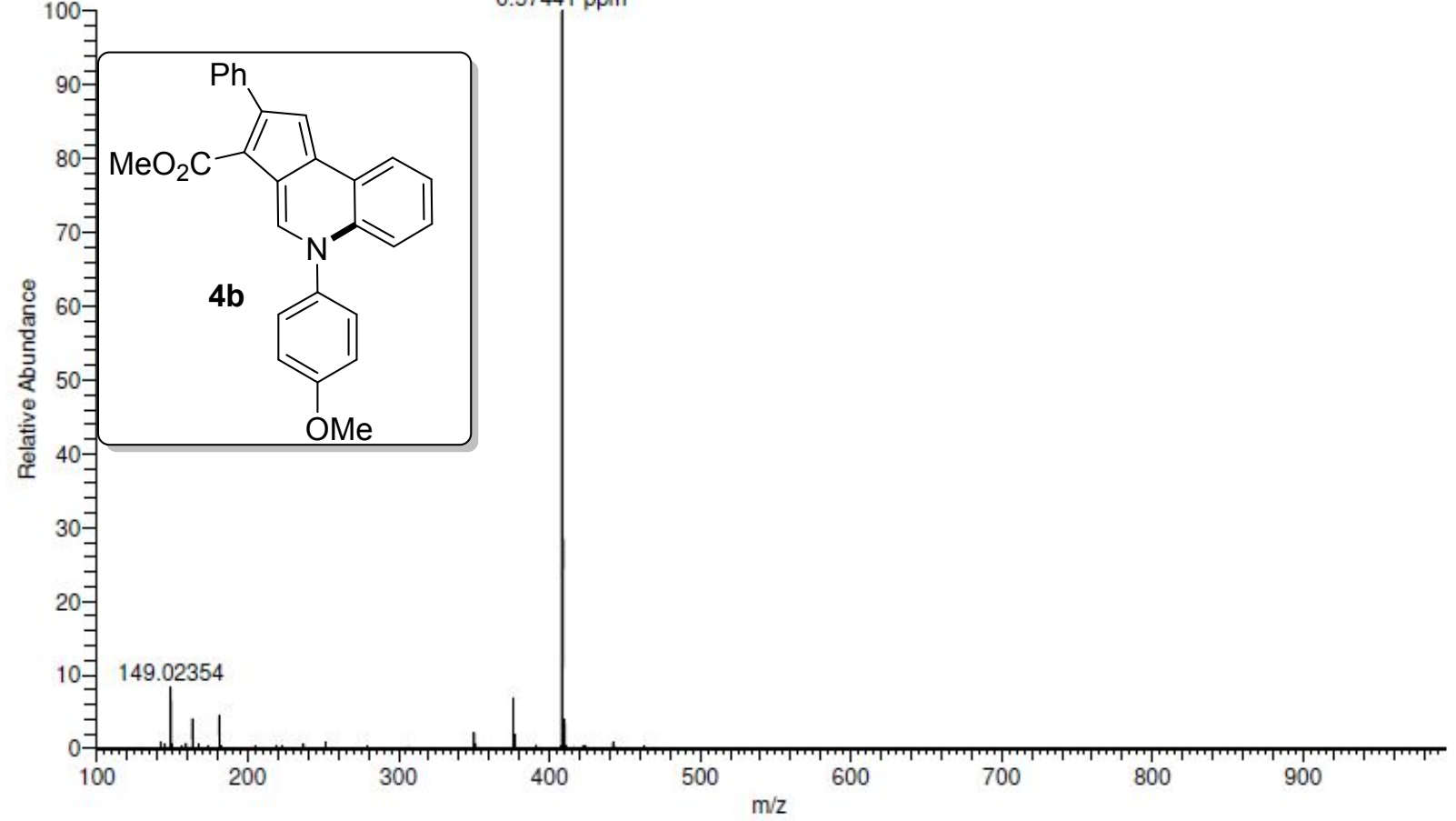




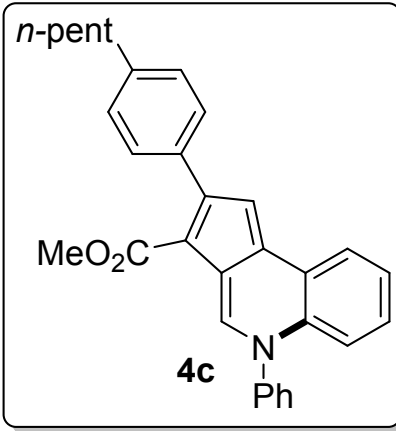

${ }^{1} \mathrm{H}$ NMR $\left(\mathrm{CDCl}_{3}, 400 \mathrm{MHz}\right)$

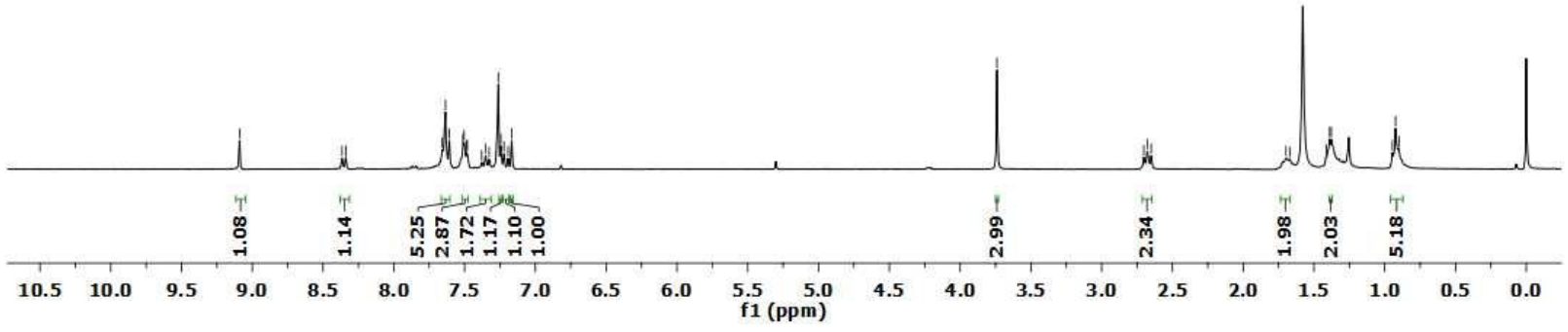

MANEESH-H0011
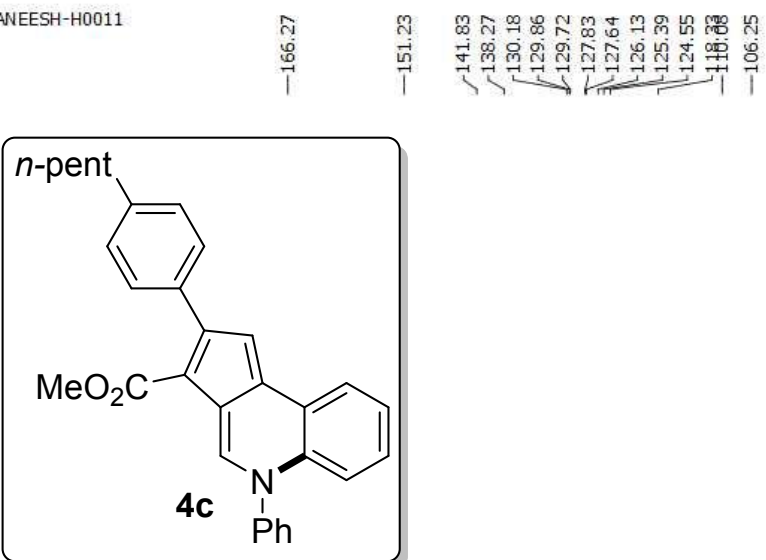

${ }^{13} \mathrm{C} \mathrm{NMR}\left(\mathrm{CDCl}_{3}, 100 \mathrm{MHz}\right)$
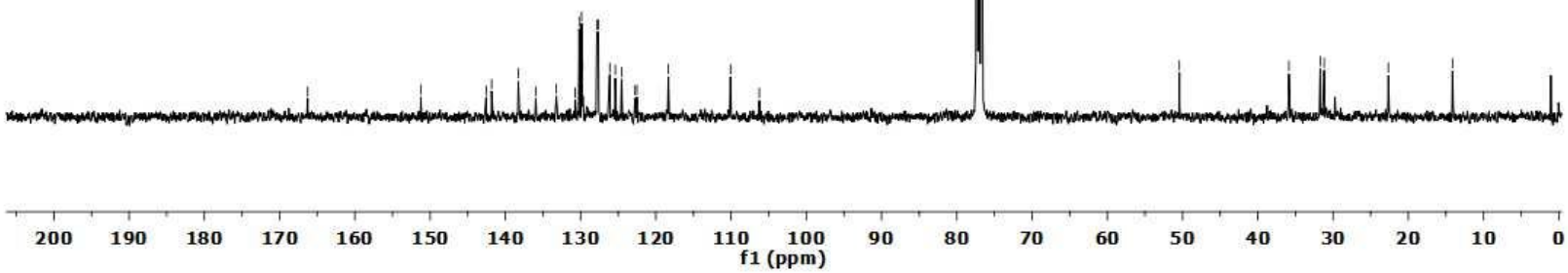


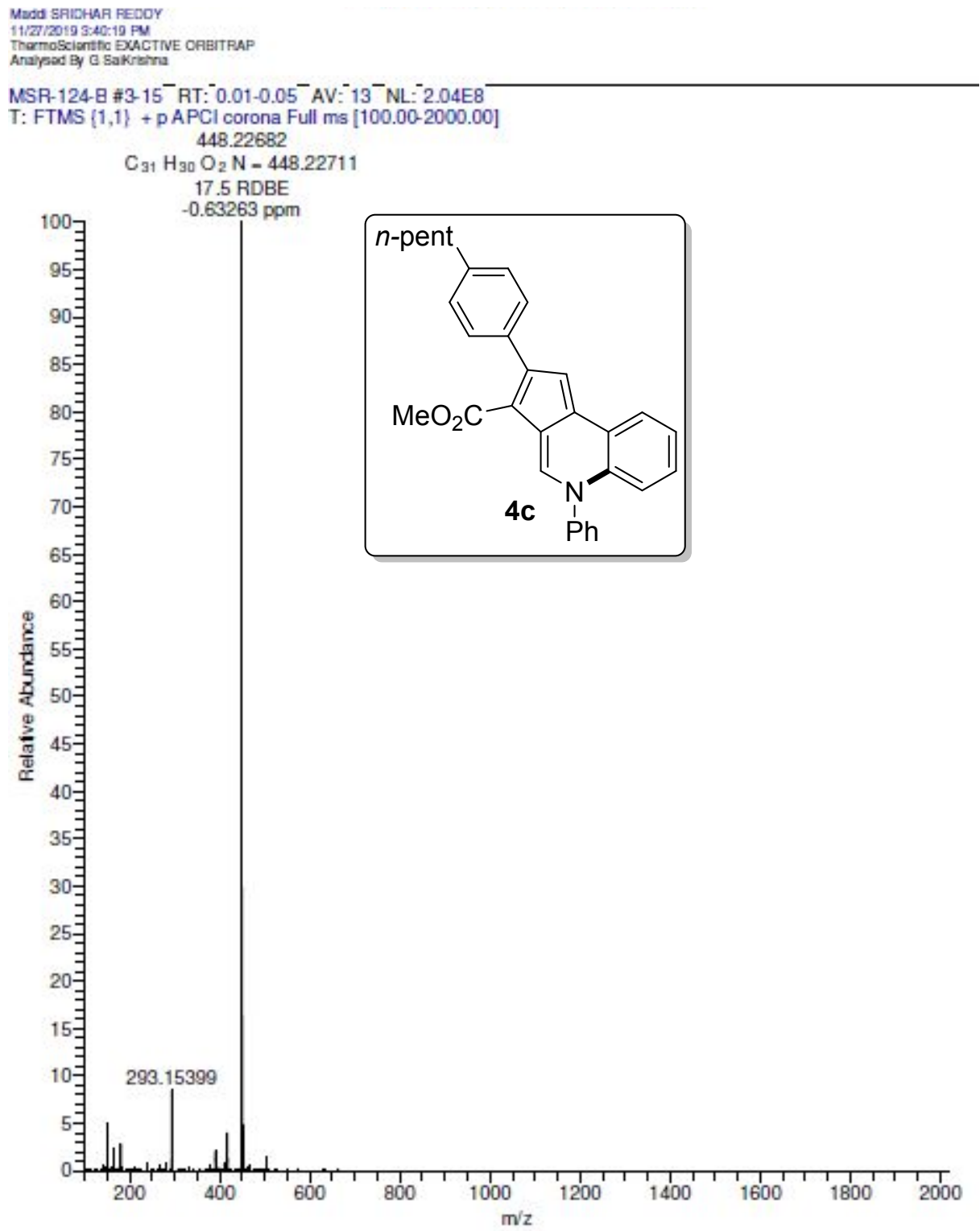




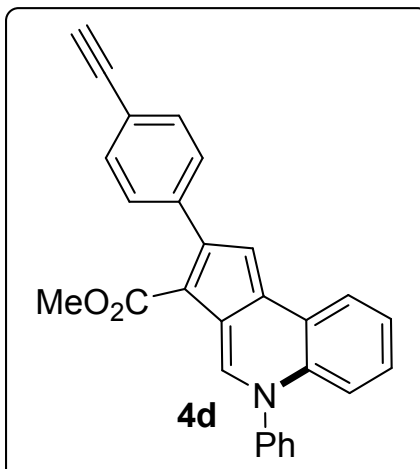

${ }^{1} \mathrm{H} \mathrm{NMR}\left(\mathrm{CDCl}_{3}, 300 \mathrm{MHz}\right)$

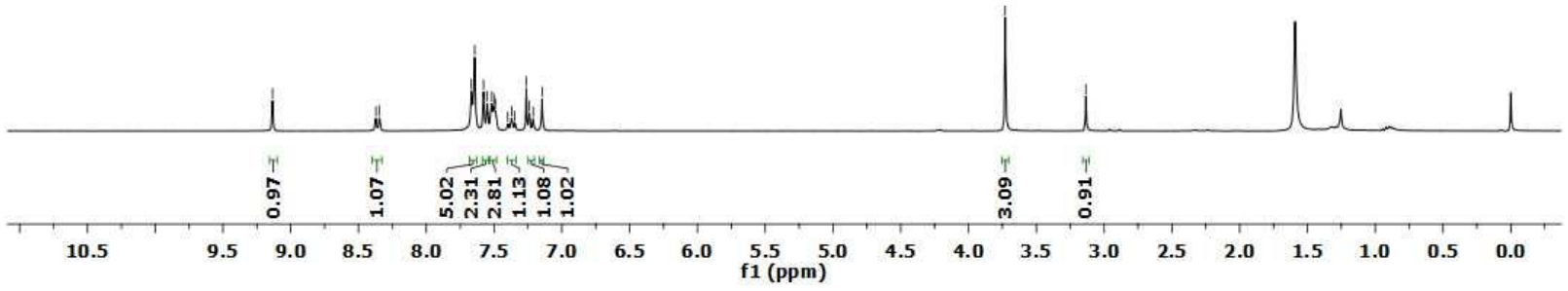

MANEESH-M0034

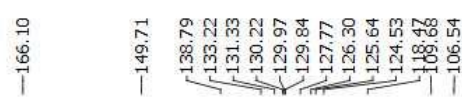

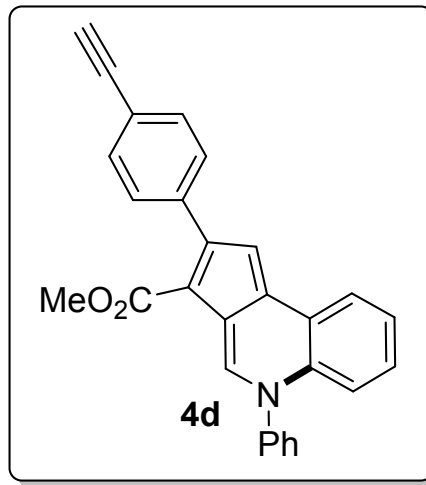

${ }^{13} \mathrm{C} \mathrm{NMR}\left(\mathrm{CDCl}_{3}, 75 \mathrm{MHz}\right)$
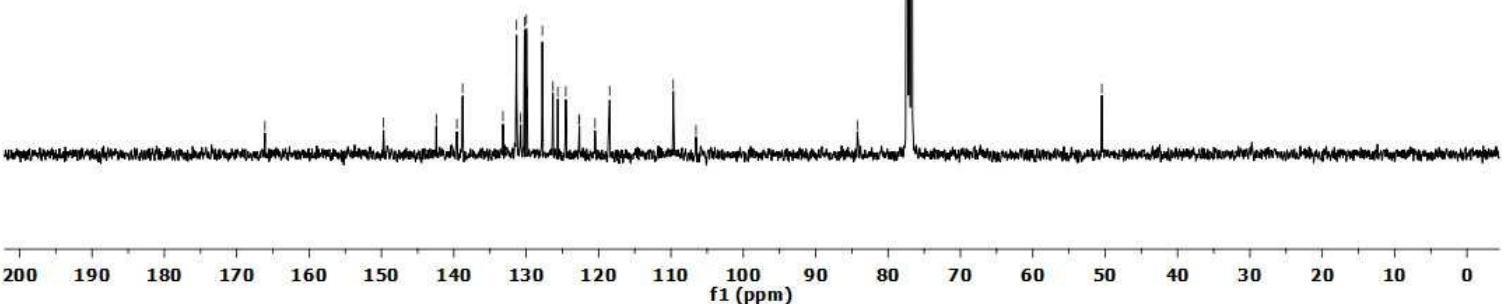
Maddi SRIDHAR REDDY

11/27/2019 3:53:09 PM

Thermoscientific EXACTIVE ORBITRAP

Analysed By G Saikrishna

msr-137-D \#2-14- ${ }^{-} \mathrm{RT}^{-}{ }^{-} 0.01-0.06^{-} \mathrm{AV}^{-}{ }^{-1}{ }^{-} \mathrm{NL}-\mathrm{C}^{-} 4.02 \mathrm{E} 8$

T: FTMS $\{1,1\}+$ p APCl corona Full ms [100.00-2000.00]

$\mathrm{C}_{28} \mathrm{H}_{20} \mathrm{O}_{2} \mathrm{~N}=402.14886$

19.5 RDBE

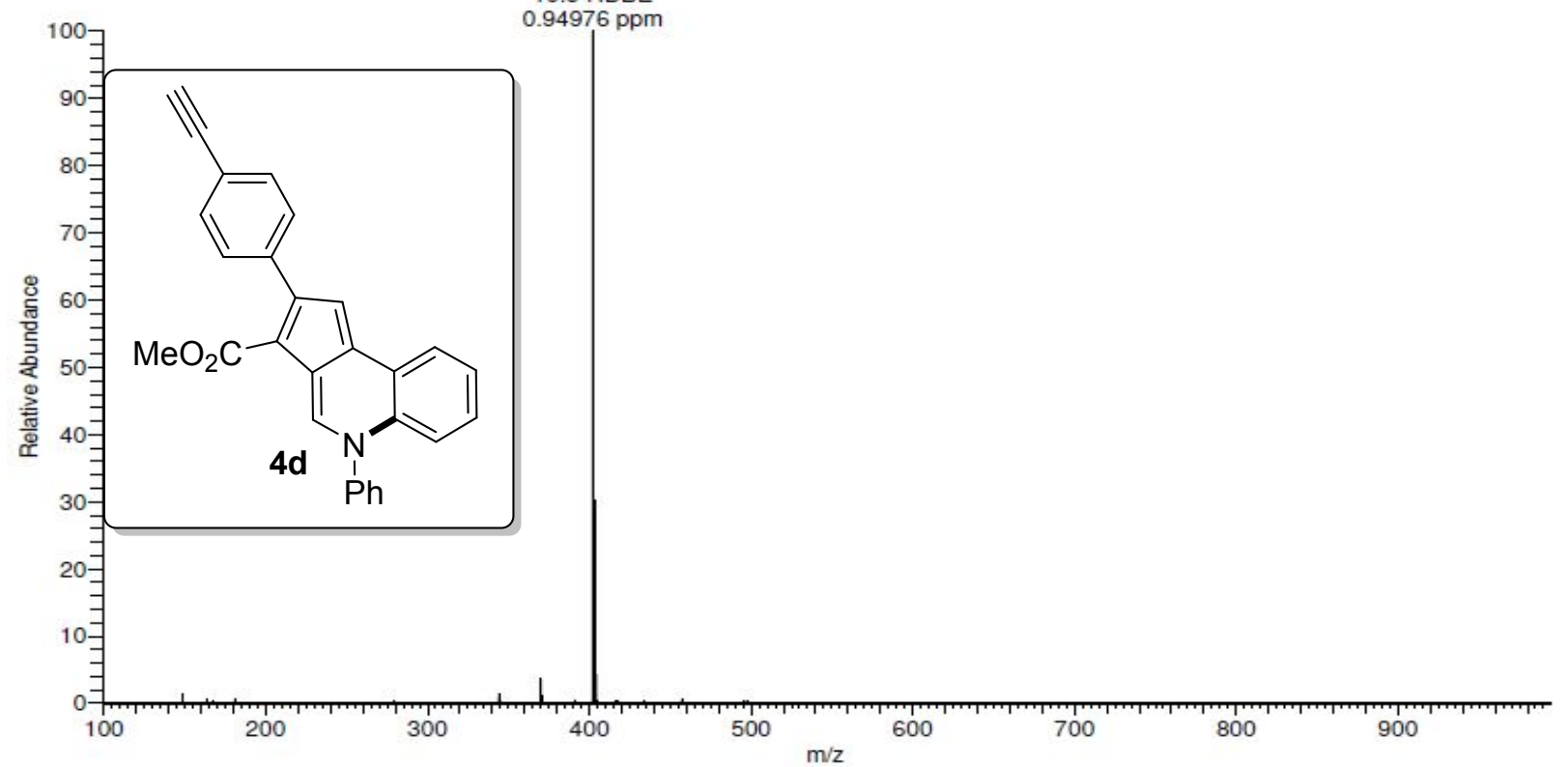

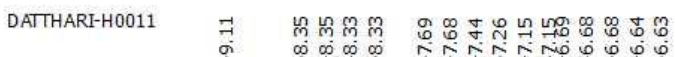

i
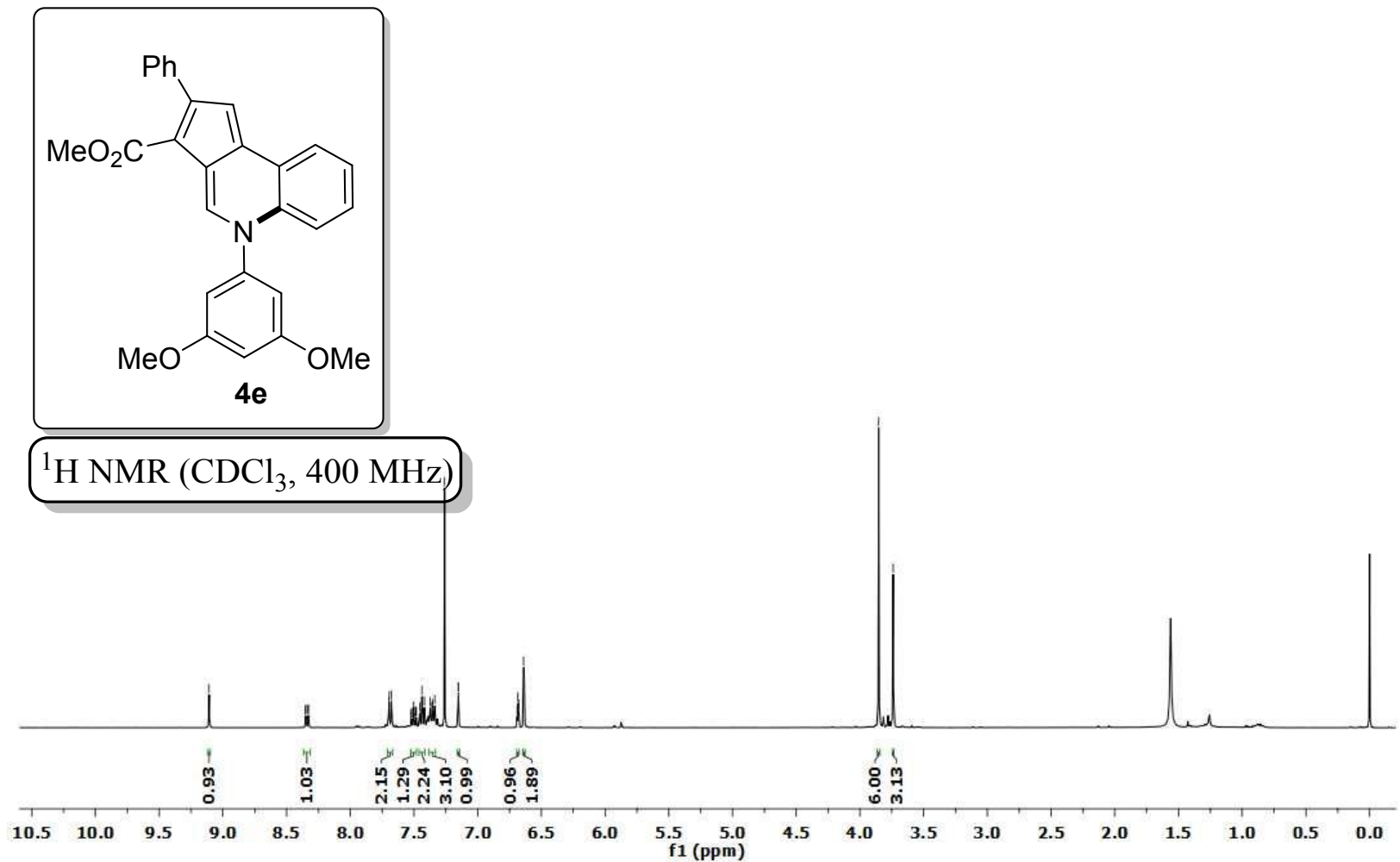
<smiles>COc1cc(OC)cc(-n2cc3c(C(C)=O)c(-c4ccccc4)cc-3c3ccccc32)c1</smiles>

${ }^{13} \mathrm{C} \mathrm{NMR}\left(\mathrm{CDCl}_{3}, 100 \mathrm{MHz}\right)$
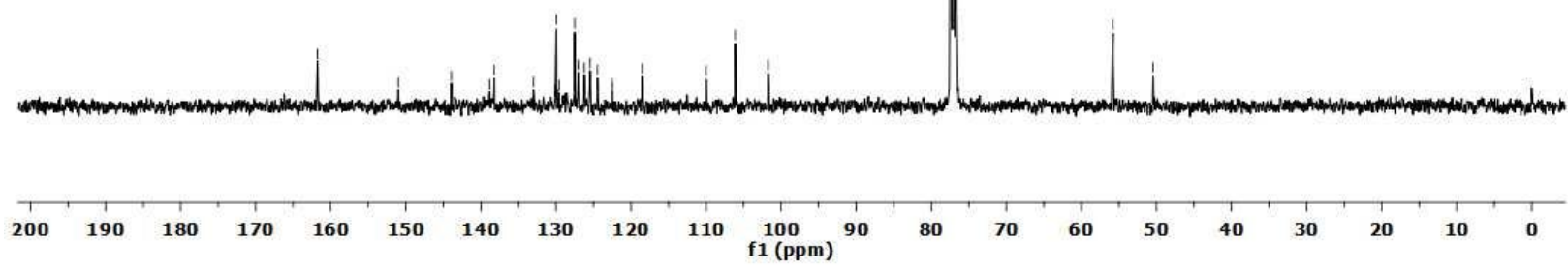

Maddi SRIDHAR REDDY

11/27/2019 4:18:53 PM

ThermoScientific EXACTIVE ORBITRAP

Analysed By $G$ Saikrishna

MSR-176-C \#1-13- ${ }^{-} \mathrm{RT}^{-}{ }^{-} 0.01-0.05^{-} \mathrm{AV}^{-}{ }^{-1}{ }^{-} \mathrm{NL}:{ }^{-} 3.42 \mathrm{E} 8$

T: FTMS $\{1,1\}+$ p APCl corona Full ms [100.00-2000.00]

438.16963

$\mathrm{C}_{28} \mathrm{H}_{24} \mathrm{O}_{4} \mathrm{~N}=438.16998$

ב $100 \quad-0.81606 \mathrm{ppm}$

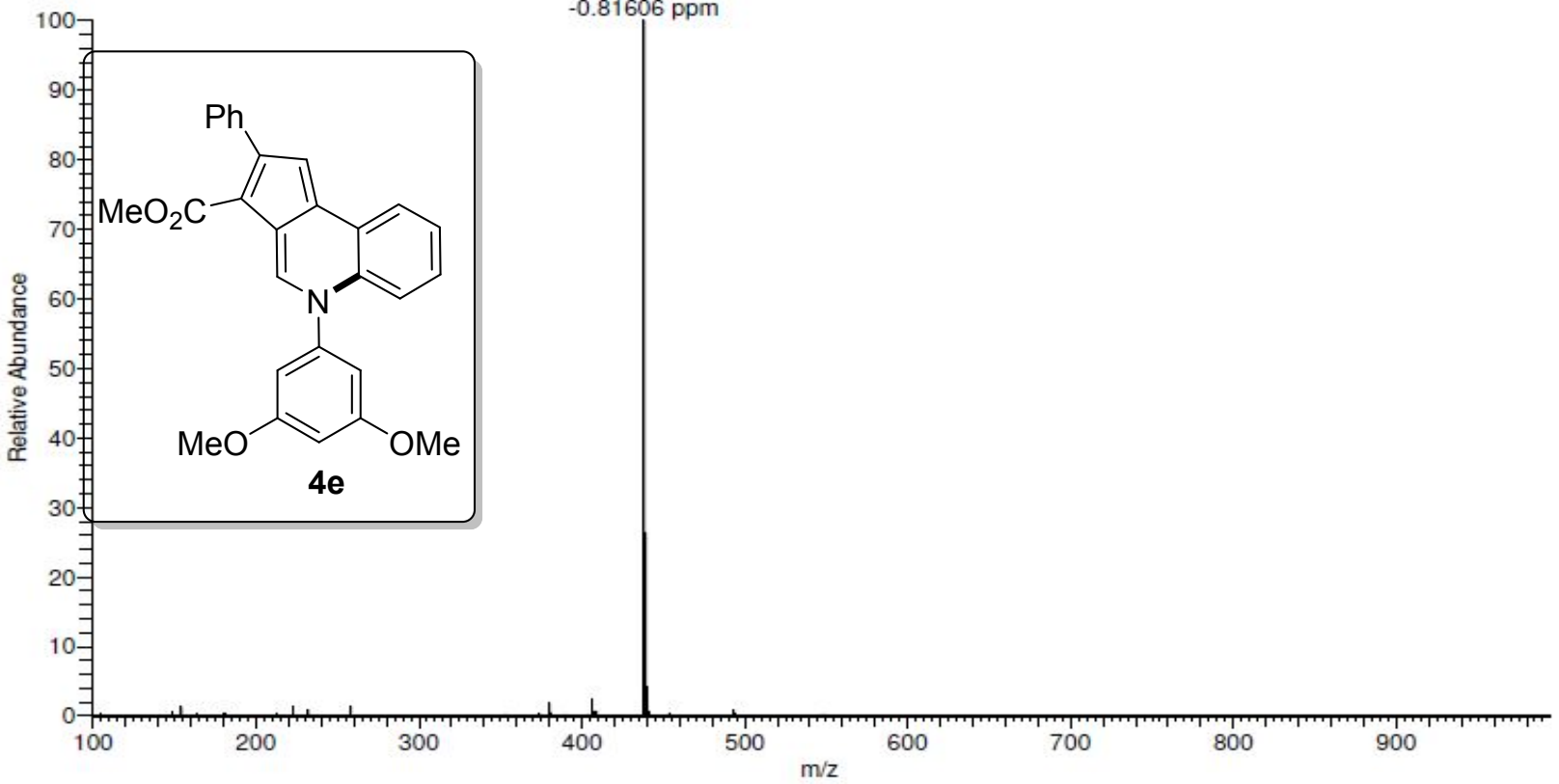




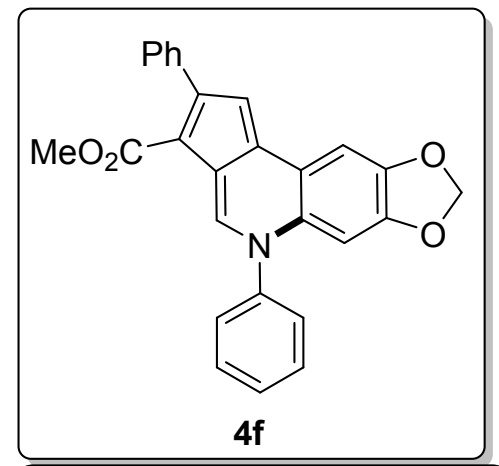

${ }^{1} \mathrm{H} \mathrm{NMR}\left(\mathrm{CDCl}_{3}, 400 \mathrm{MHz}\right)$

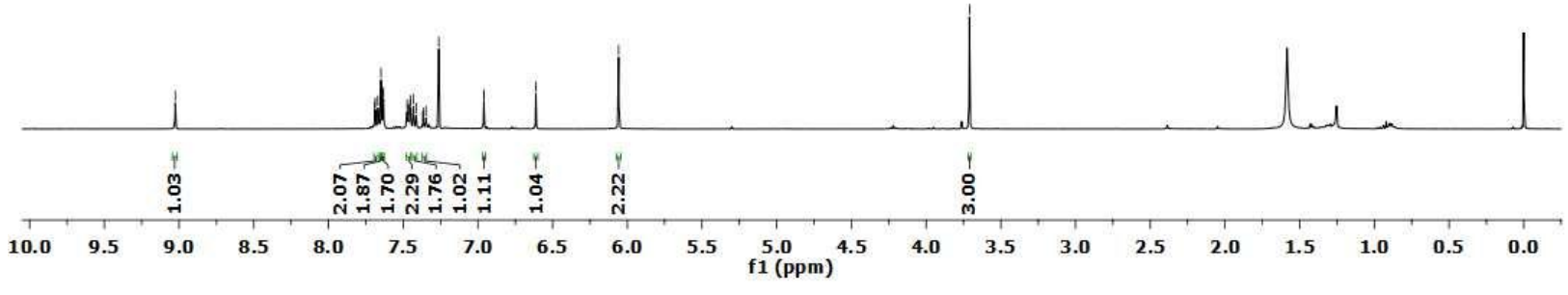

MANEESH-H0011

ᄁ

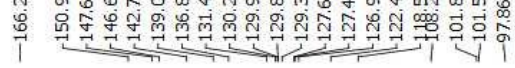

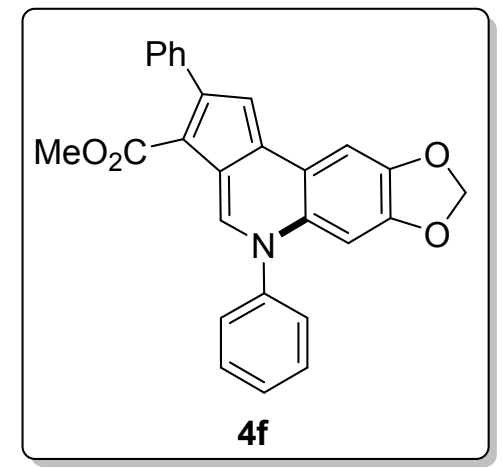

${ }^{13} \mathrm{C} \mathrm{NMR}\left(\mathrm{CDCl}_{3}, 100 \mathrm{MHz}\right)$
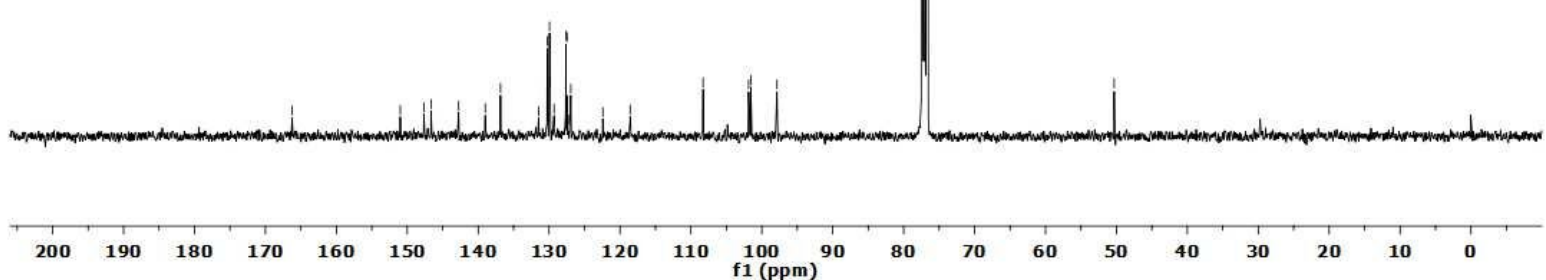
D:ISai krishna Importantl....MSR-129-A 118049426

Maddi SRIDHAR REDDY

11/27/2019 4.24:01 PM

Thermoscientific EXACTIVE ORBITRAP

Analysed By $G$ Saikrishna

MSR-129-A \#1-13- ${ }^{-}$RT: ${ }^{-0} 0.01-0.05^{-}{ }^{-}{ }^{-}{ }^{-1}{ }^{-}$NL: ${ }^{-} 7.66 \mathrm{E} 7$

T: FTMS $\{1,1\}+\mathrm{p} \mathrm{APCl}$ corona Full ms [100.00-2000.00] 422.13884

$\mathrm{C}_{27} \mathrm{H}_{20} \mathrm{O}_{4} \mathrm{~N}=422.13868$
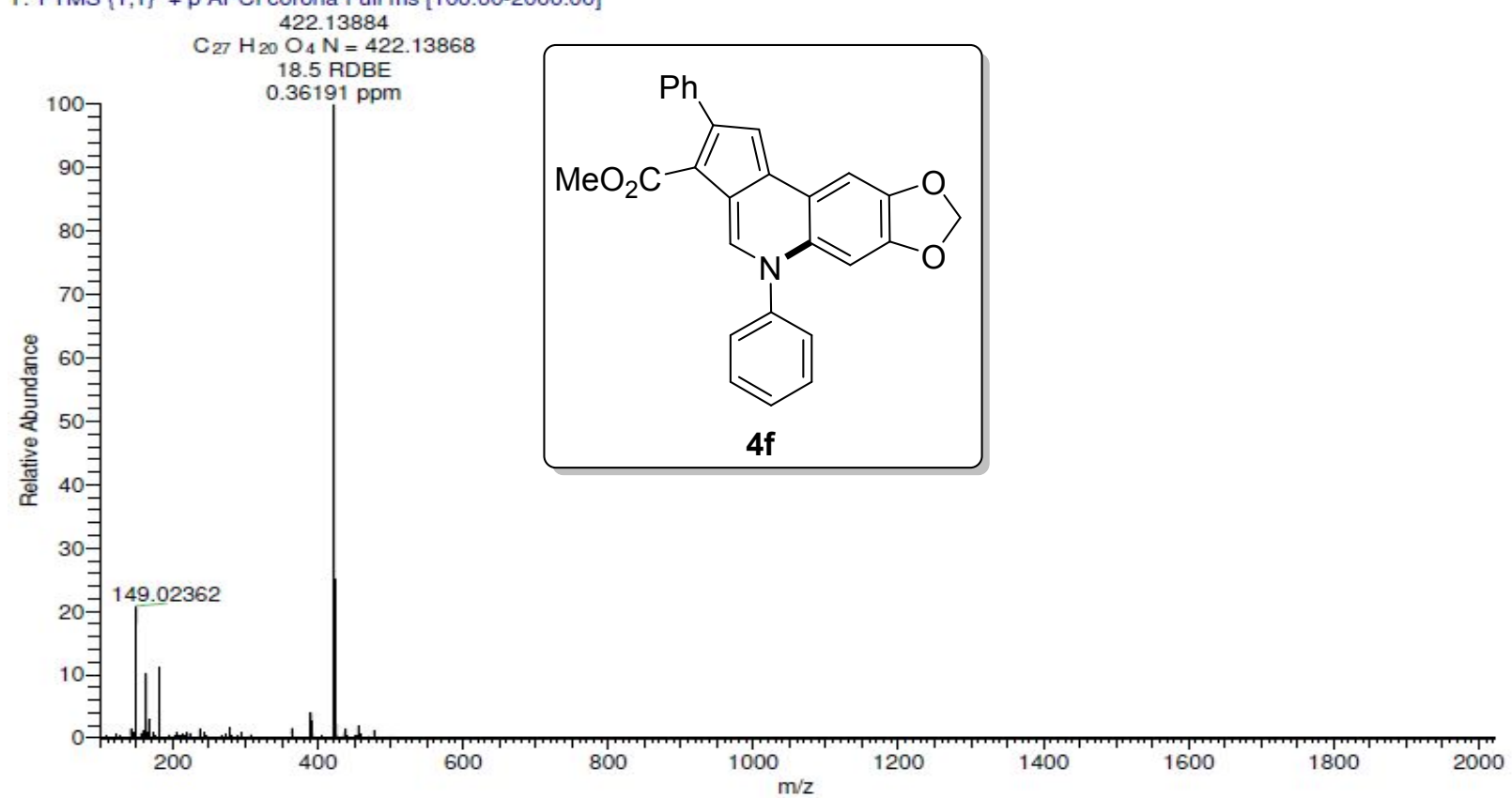

CSIR-INDIAN INSTITUTE OF CHEMICAL TECHNOLOGY ANALYTICAL CHEMISTRY AND MASS SPECTROMETRY

MANEESH-H0011

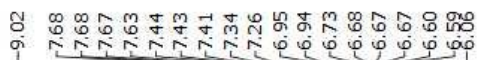

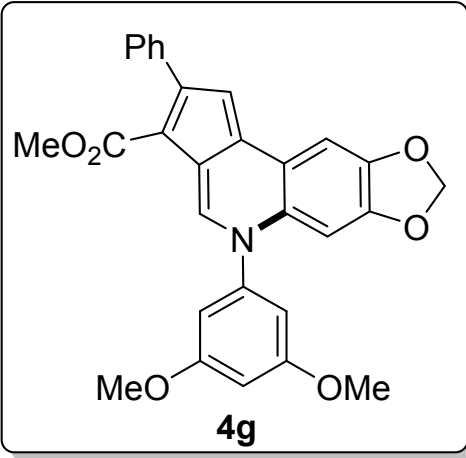

${ }^{1} \mathrm{H}$ NMR $\left(\mathrm{CDCl}_{3}, 400 \mathrm{MHz}\right)$

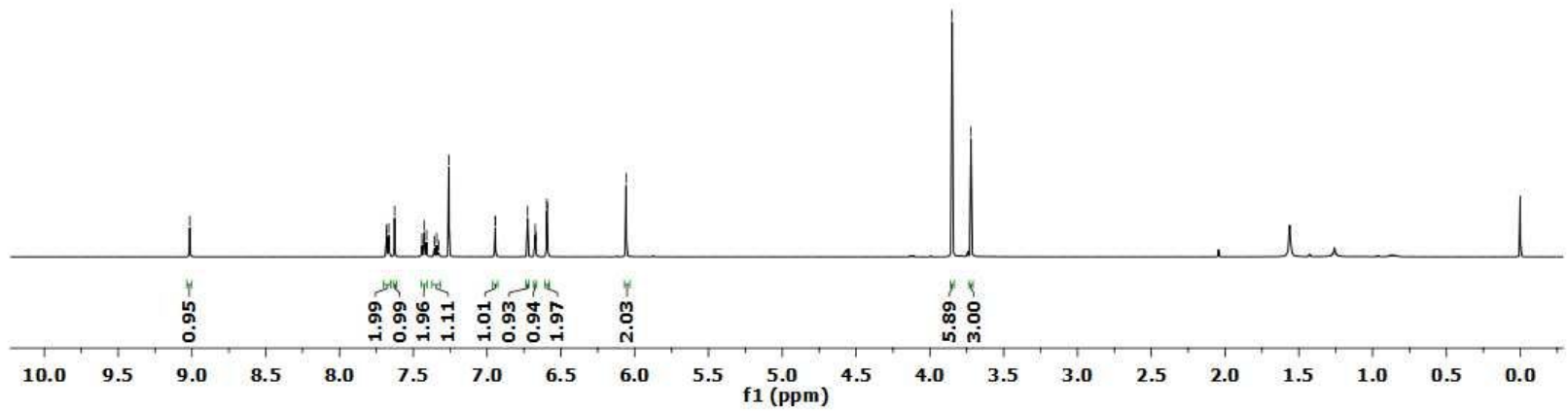


NAGIREDDY-H0011

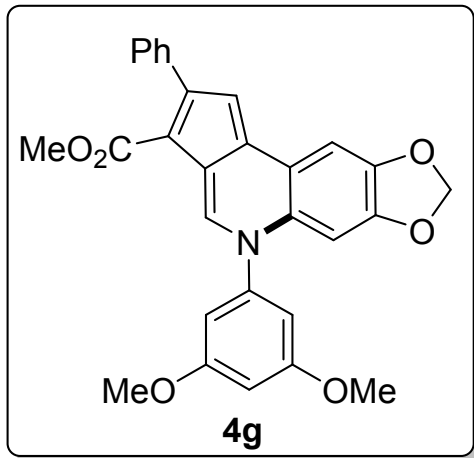

${ }^{13} \mathrm{C} \mathrm{NMR}\left(\mathrm{CDCl}_{3}, 100 \mathrm{MHz}\right)$
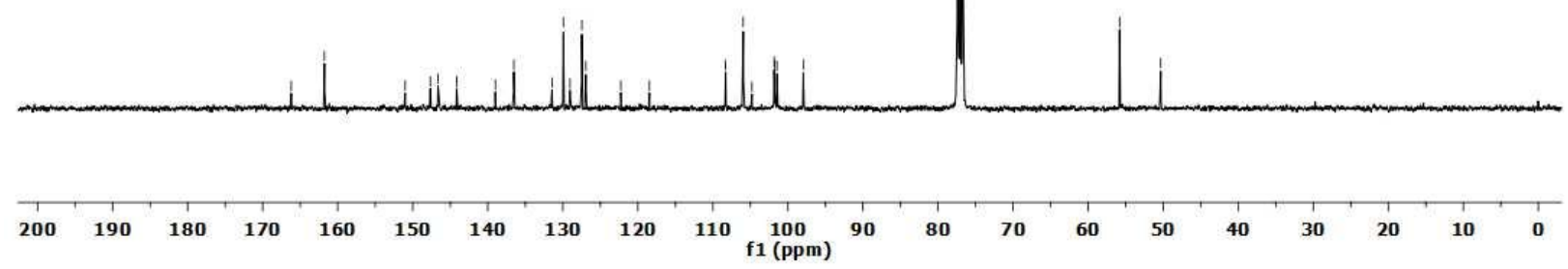

Maddi SRIDHAR REDDY

11/27/2019 4:16:18 PM

ThermoScientific EXACTIVE ORBITRAP

Analysed By G Saikrishna

MSR-176-B \#1-13 ${ }^{-}$RT: $:^{-} 0.01-0.05^{-} \mathrm{AV}^{-} 13^{-} \mathrm{NL}:{ }^{-} 1.54 \mathrm{E} 8$

T: FTMS $\{1,1\}+p$ APCl corona Full ms [100.00-2000.00]

$$
\begin{gathered}
482.15954 \\
\mathrm{C}_{29} \mathrm{H}_{24} \mathrm{O}_{6} \mathrm{~N}=482.15981 \\
18.5 \mathrm{RDBE}
\end{gathered}
$$

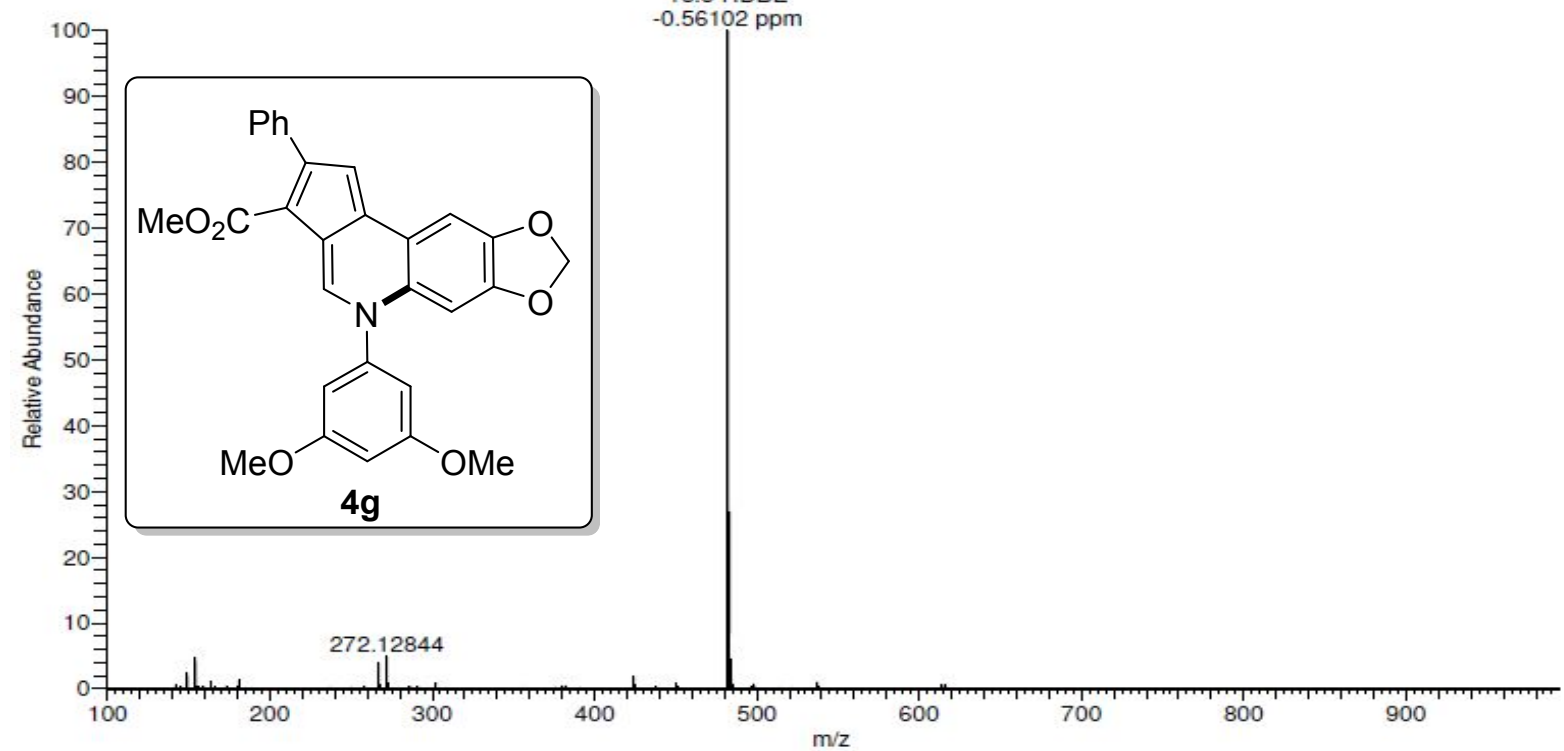




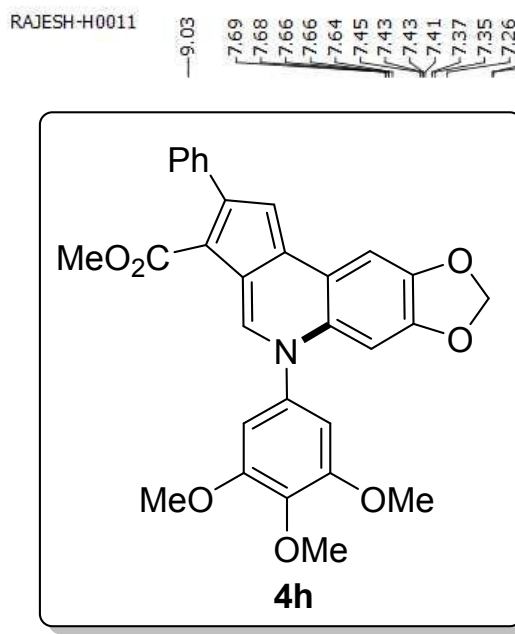

${ }^{1} \mathrm{H}$ NMR $\left(\mathrm{CDCl}_{3}, 400 \mathrm{MHz}\right)$
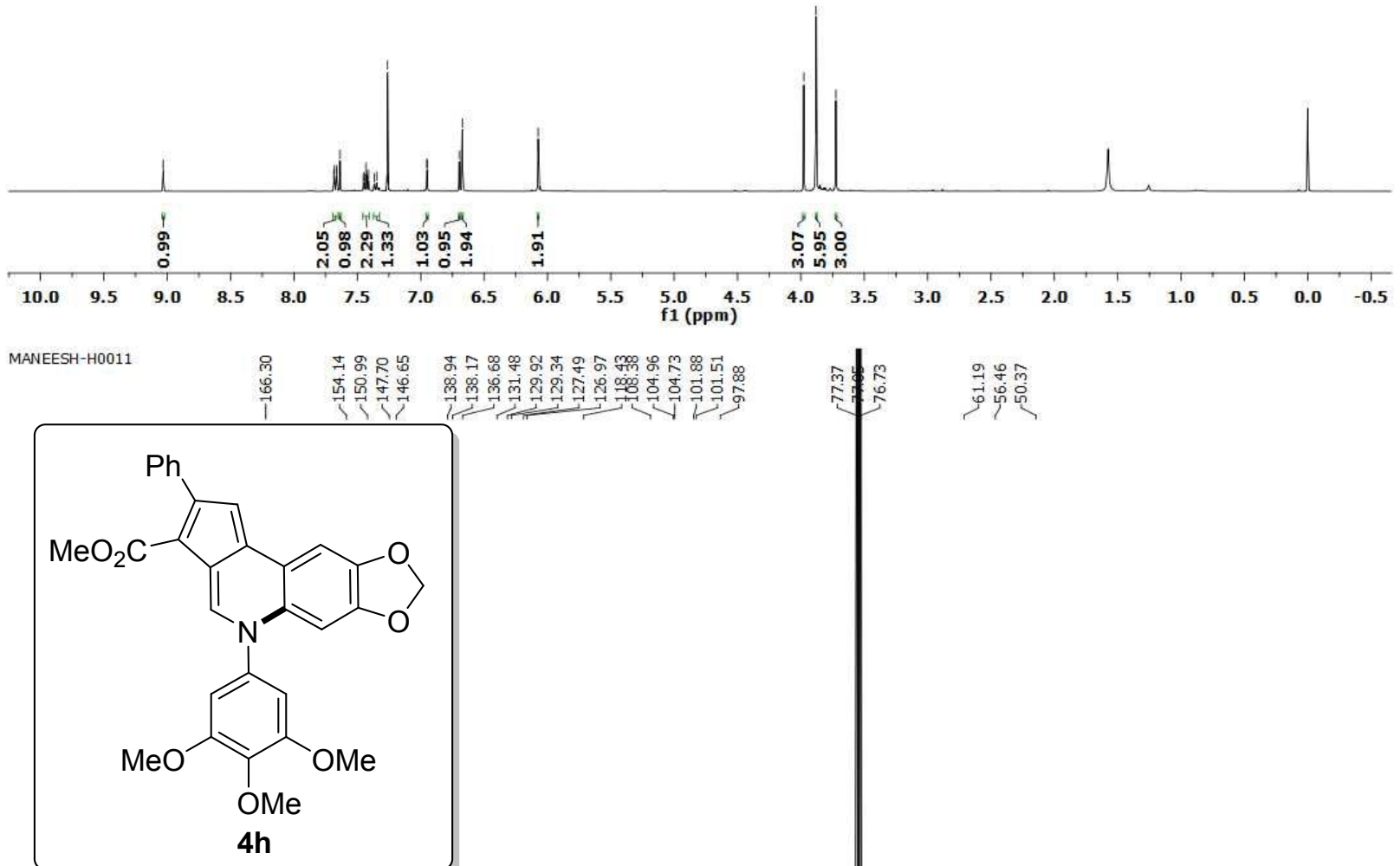

${ }^{13} \mathrm{C}$ NMR $\left(\mathrm{CDCl}_{3}, 100 \mathrm{MHz}\right)$

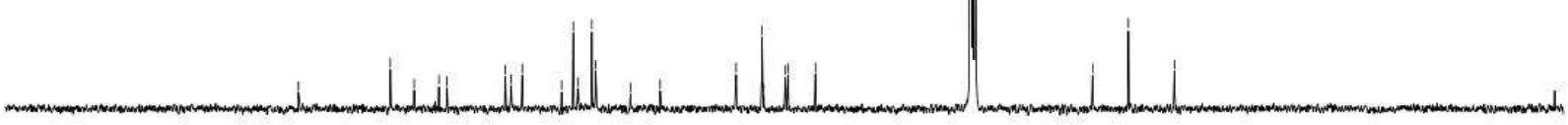




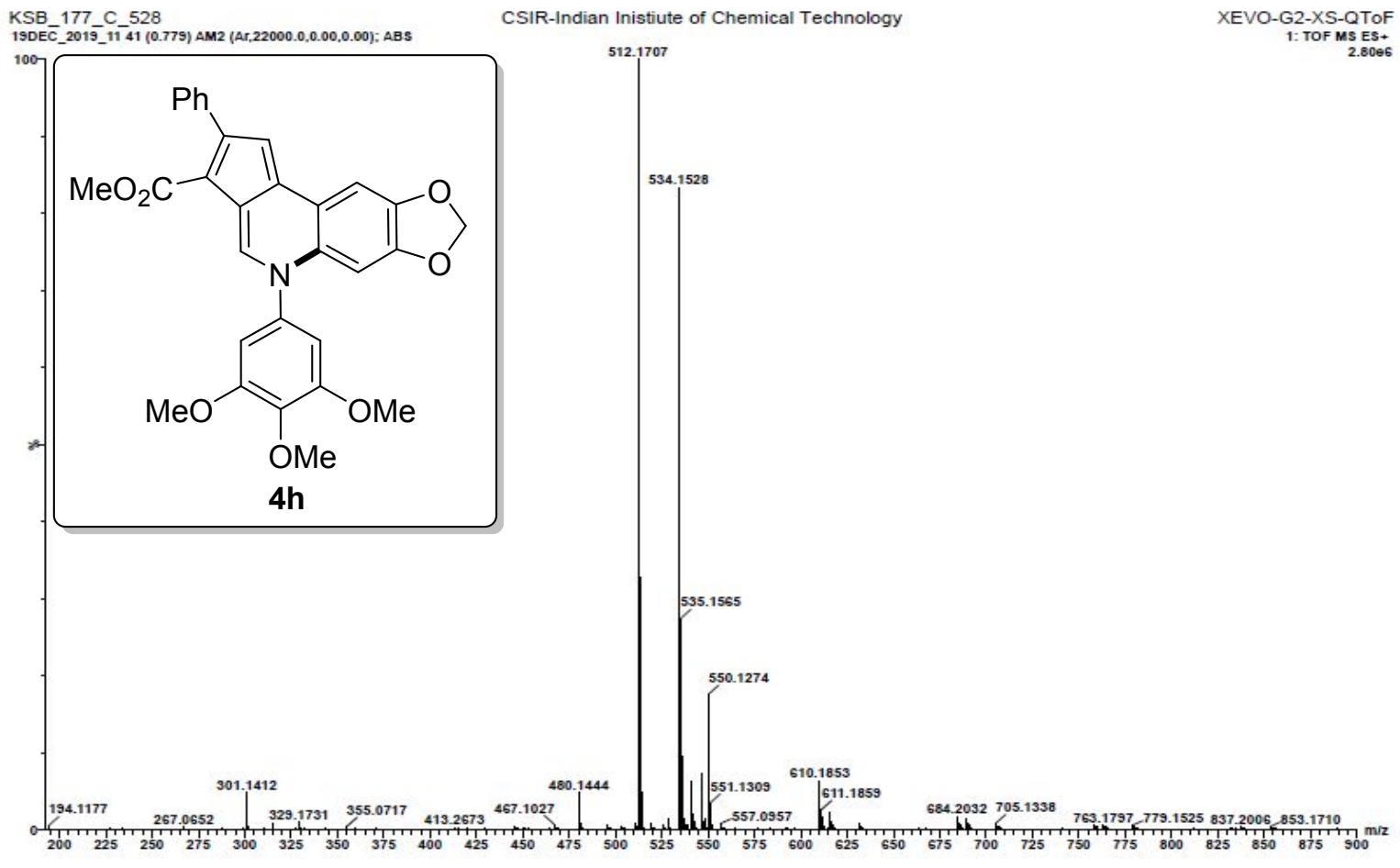




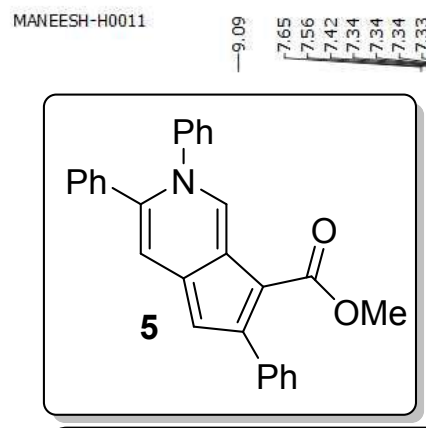

${ }^{1} \mathrm{H} \mathrm{NMR}\left(\mathrm{CDCl}_{3}, 500 \mathrm{MHz}\right)$

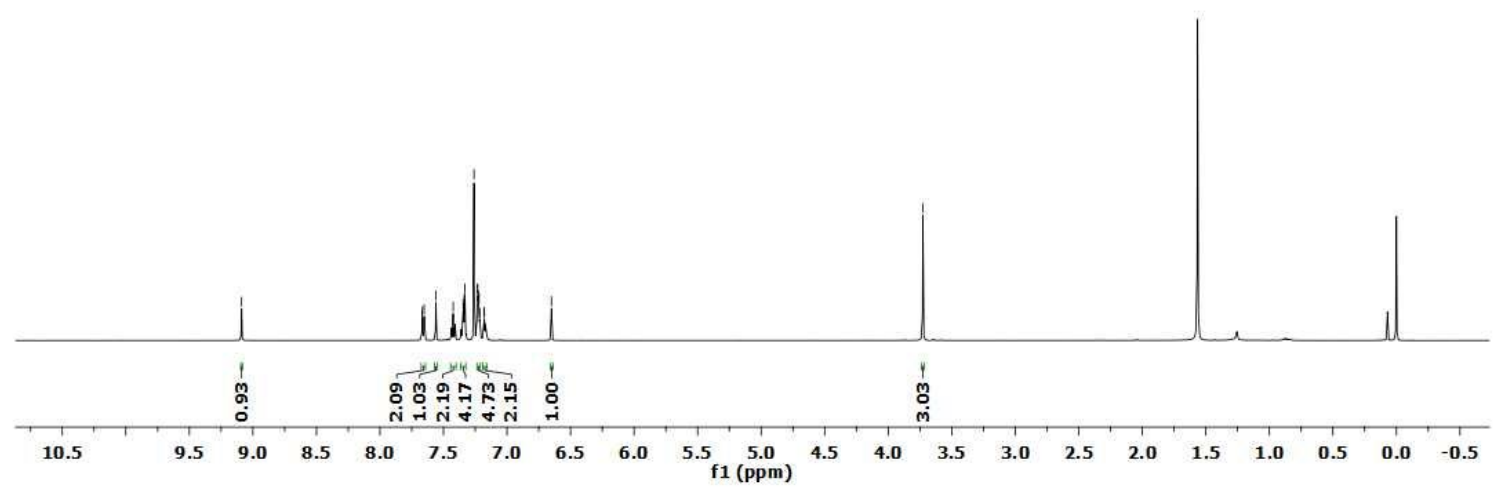

MANEESH-H0011
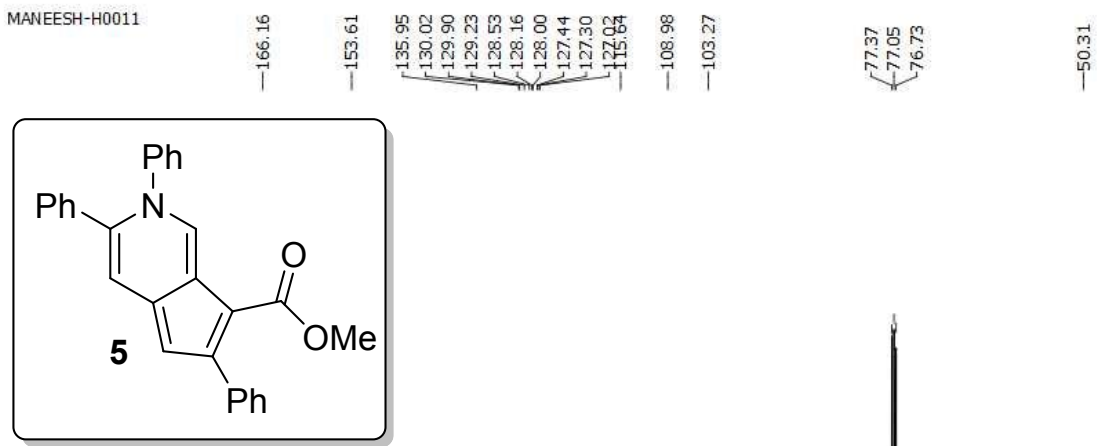

${ }^{13} \mathrm{C} \mathrm{NMR}\left(\mathrm{CDCl}_{3}, 125 \mathrm{MHz}\right)$
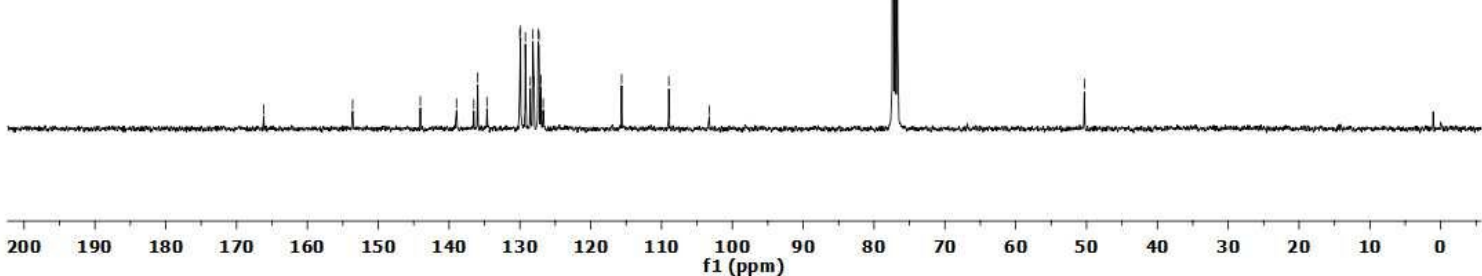


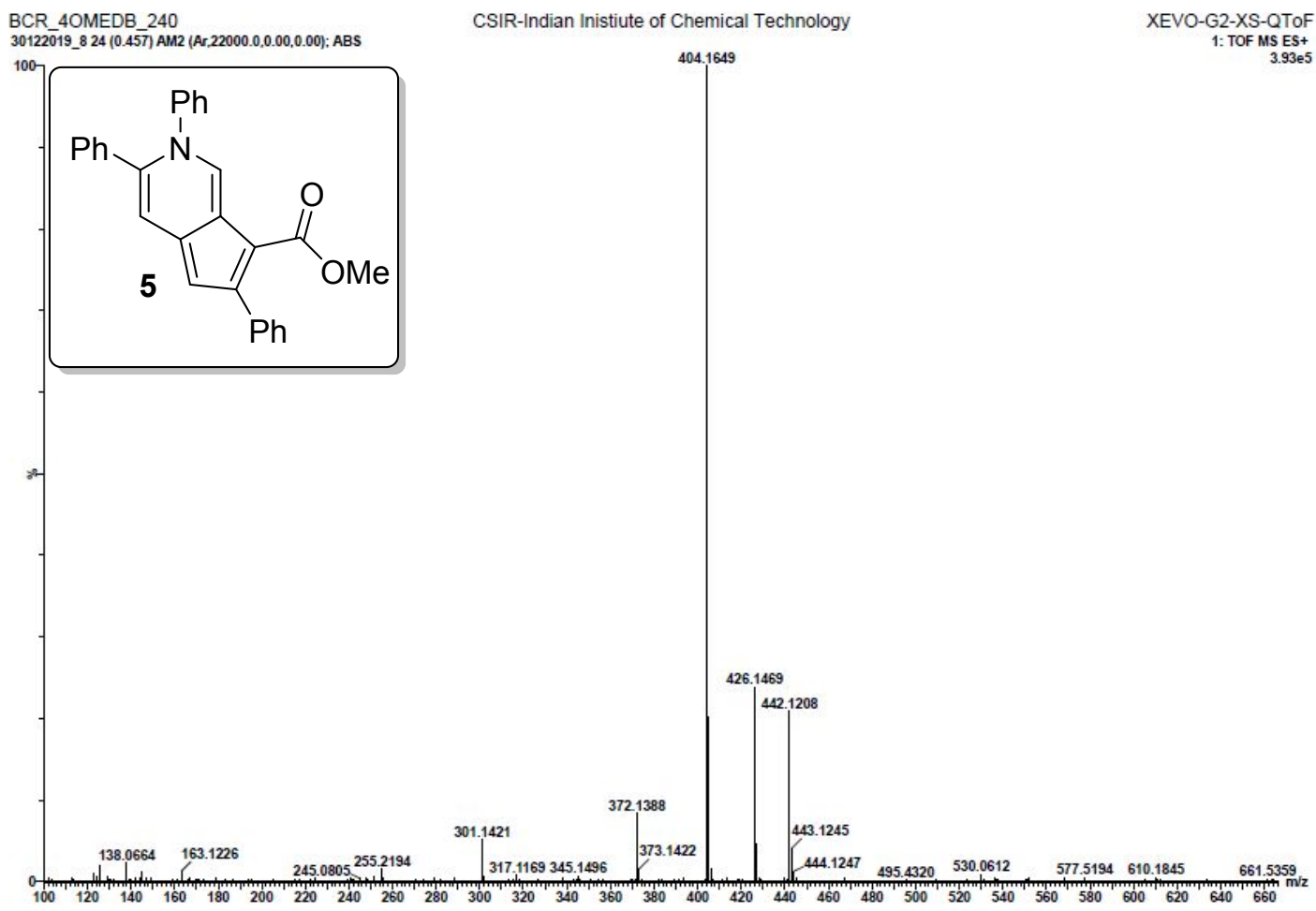




\section{Crystallographic data}

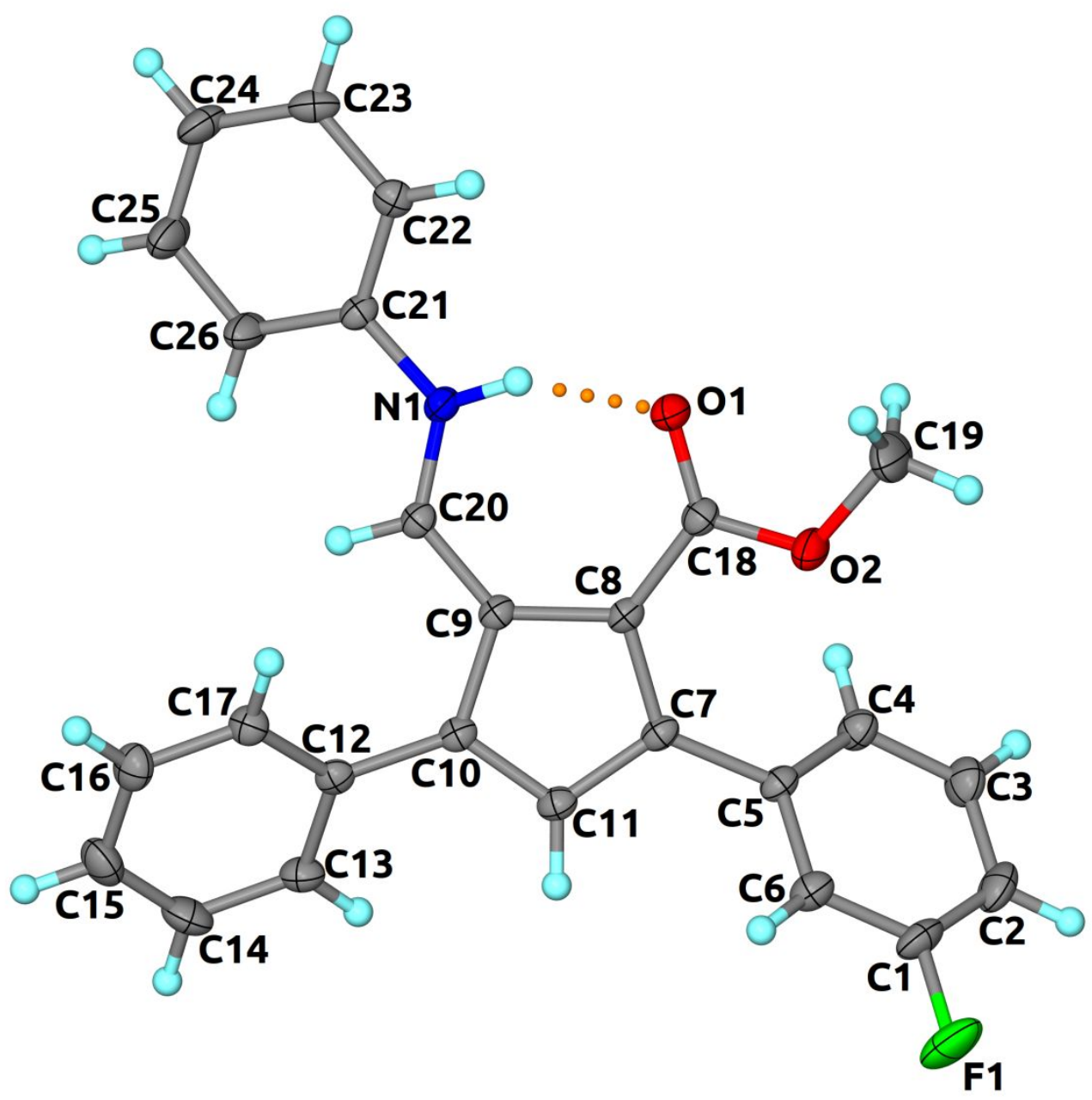

Figure caption: ORTEP diagram of KA831 compound with the atom-numbering. Displacement ellipsoids are drawn at the $35 \%$ probability level and $\mathrm{H}$ atoms are shown as small spheres of arbitrary radius. CCDC 1972696 contains the supplementary crystallographic data for this paper which can be obtained free of charge at https://www.ccdc.cam.ac.uk/structures/

Sample Preparation for Crystal Growth: The compound $\mathbf{3 f a}$ was dissolved in $\mathrm{CDCl}_{3}$ in beaker and kept for slow evaporation at room temperature. Formation of needle shape crystals was observed five days. The single crystals were then subjected to X-ray diffraction analysis. 
Table S1.Crystal Data Collection and Refinement Parameters for Complex 1.

\begin{tabular}{|c|c|}
\hline & KA831 \\
\hline chemical formula & $\mathrm{C}_{26} \mathrm{H}_{20} \mathrm{FNO}_{2}$ \\
\hline$F w ; F(000)$ & $397.43 ; 832$ \\
\hline$T(\mathrm{~K})$ & $293(2)$ \\
\hline wavelength (Å) & 0.71073 \\
\hline space group & $\mathrm{P} 21 / \mathrm{n}$ \\
\hline$a(\AA)$ & $8.920(8)$ \\
\hline$b(\AA)$ & $13.754(12)$ \\
\hline$c(\AA)$ & $17.019(17)$ \\
\hline$\alpha(\operatorname{deg})$ & 90 \\
\hline$\beta(\operatorname{deg})$ & $103.67(2)$ \\
\hline$\gamma(\operatorname{deg})$ & 90 \\
\hline$Z$ & 4 \\
\hline$V\left(\AA^{3}\right)$ & 2029(3) \\
\hline$\rho_{\text {calcd }}\left(\mathrm{g} \cdot \mathrm{cm}^{-3}\right)$ & 1.301 \\
\hline$\mu\left(\mathrm{mm}^{-1}\right)$ & 0.088 \\
\hline$\theta$ range (deg); completeness & $2.381-27.500 ; 0.999$ \\
\hline Collected reflections; $\mathbf{R}_{\sigma}$ & $22775 ; 0.0636$ \\
\hline Unique reflections; $\mathbf{R}_{\text {int }}$ & $22775 ; 0.0753$ \\
\hline $\mathrm{R}^{\mathrm{a}} ; \mathrm{wR2}^{\mathrm{b}}[\mathrm{I}>\mathbf{2 \sigma} \sigma(\mathrm{I})]$ & $0.0580 ; 0.1613$ \\
\hline R1; wR2 [all data] & $0.0855 ; 0.1811$ \\
\hline GOF & 1.161 \\
\hline Largest diff peak and hole & 0.236 and -0.243 \\
\hline
\end{tabular}

${ }^{\mathrm{a}} R_{1}=\Sigma\left(|| F_{o}|-| F_{c}||\right) / \Sigma\left|F_{o}\right|$

b

$\mathrm{wR}_{2}=\left\{\boldsymbol{\Sigma}\left[\mathrm{w}\left(\mathbf{F}_{\mathbf{0}}{ }^{2}-\mathbf{F}_{\mathrm{c}}{ }^{2}\right)^{2}\right] / \Sigma\left[\mathrm{w}\left(\mathbf{F}_{\mathbf{0}}{ }^{2}\right)^{2}\right]\right\}^{1 / 2}$ 
Data collection and Structure solution details: Single crystal X-ray data for KA831 compound were collected at room temperature on a Bruker D8 QUEST equipped with a four-circle kappa diffractometer and Photon 100 detector. An I $\mu$ smicrofocus Mo source $(\lambda=0.71073 \AA)$ supplied the multi-mirror monochromated incident beam. A combination of Phi and Omega scans were used to collect the necessary data and unit cell dimensions were determined using 9971 reflections. Integration and scaling of intensity data were accomplished using SAINT program. ${ }^{1}$ The structures were solved by Direct Methods using SHELXS972 and refinement was carried out by full-matrix least-squares technique using SHELXL-2014/7. ${ }^{2-3}$ Anisotropic displacement parameters were included for all non-hydrogen atoms. All $\mathrm{H}$ atoms were positioned geometrically and treated as riding on their parent $\mathrm{C}$ atoms, with $\mathrm{C}-\mathrm{H}$ distances of 0.93--0.97 $\AA$, and with $U_{\text {iso }}(\mathrm{H})=1.2 U_{\text {eq }}(\mathrm{C})$ or $1.5 U_{\text {eq }}$ for methyl atoms. The $\mathrm{N}$ bound $\mathrm{H}$ atoms were located from the difference Fourier map.

1. SMART \& SAINT. Software Reference manuals. Versions 6.28a \& 5.625, Bruker Analytical Xray Systems Inc., Madison, Wisconsin, U.S.A., 2001.

2. Sheldrick, G. M. SHELXS97 and SHELXL Version 2014/7, http://shelx.uniac.gwdg.de/SHELX/index.php

3. Muller, P, Herbst-Imer, R, Spek, A. L, Schneider, T. R, and Sawaya, M. R. Crystal Structure Refinement: A Crystallographer's Guide to SHELXL. Muller, P. Ed. 2006 Oxford University Press: Oxford, New York, pp. 57-91.

4. A. L. Spek. Structure validation in chemical crystallography. ActaCryst. 2009, D65, 148-155. 